In cooperation with the National Park Service, U.S. Department of Agriculture Forest Service, Teton County, Wyoming, and

Colorado Department of Public Health and Environment

\title{
Rocky Mountain Snowpack Physical and Chemical Data for Selected Sites, 1993-2008
}

Data Series 369

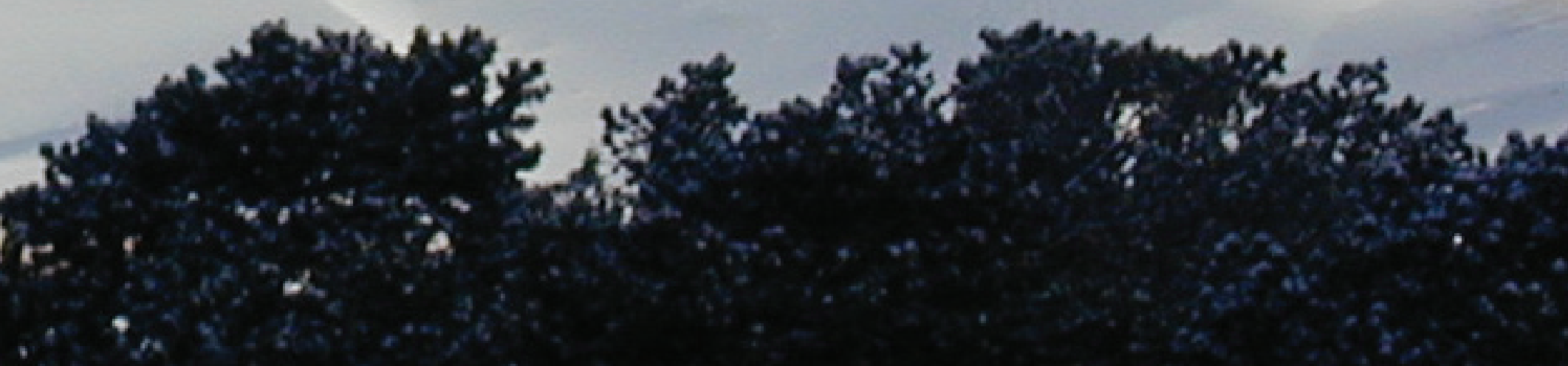

U.S. Department of the Interior U.S. Geological Survey 
Cover photograph: Spring snowstorm transforms the Great Sand Dunes, March 2006. Photograph by George P. Ingersoll, U.S. Geological Survey. 


\section{Rocky Mountain Snowpack Physical and Chemical Data for Selected Sites, 1993-2008}

By George P. Ingersoll, M. Alisa Mast, Donald H. Campbell, David W. Clow, Leora Nanus, and John T. Turk

In cooperation with the National Park Service, U.S. Department of Agriculture Forest Service,

Teton County, Wyoming, and

Colorado Department of Public Health and Environment

Data Series 369 


\section{U.S. Department of the Interior \\ KEN SALAZAR, Secretary}

\section{U.S. Geological Survey \\ Suzette M. Kimball, Acting Director}

\section{U.S. Geological Survey, Reston, Virginia: 2009}

For product and ordering information:

World Wide Web: http://www.usgs.gov/pubprod

Telephone: 1-888-ASK-USGS

For more information on the USGS —-the Federal source for science about the Earth, its natural and living resources, natural hazards, and the environment:

World Wide Web: http://www.usgs.gov

Telephone: 1-888-ASK-USGS

Any use of trade, product, or firm names is for descriptive purposes only and does not imply endorsement by the U.S. Government.

Although this report is in the public domain, permission must be secured from the individual copyright owners to reproduce any copyrighted materials contained within this report.

Suggested citation:

Ingersoll, G.P., Mast, M.A., Campbell, D.H., Clow, D.W., Nanus, L., and Turk, J.T., 2009, Rocky Mountain snowpack physical and chemical data for selected sites, 1993-2008: U.S. Geological Survey Data Series 369, 90 p. 


\section{Contents}

Abstract
Introduction
Purpose and Scope

\section{Figure}

1. Study area and snowpack-sampling sites in Rocky Mountain region of the United States

\section{Tables}

1. Station ID and snow-sampling-site location information .................................................

2-17. Selected chemical and physical data for Rocky Mountain snowpack samples for:

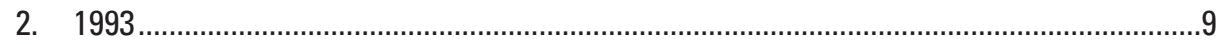

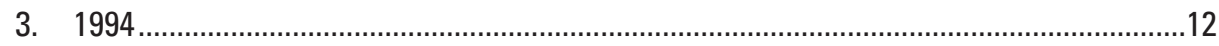

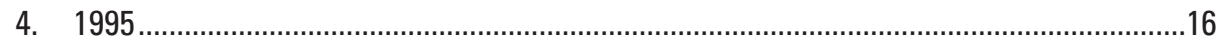

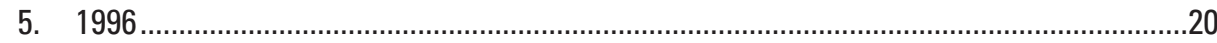

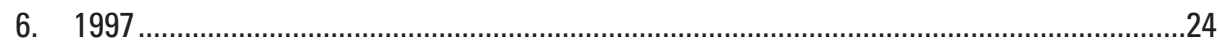

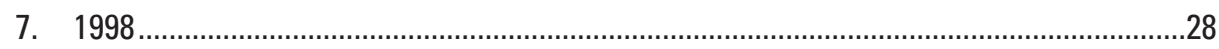

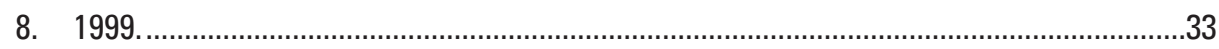

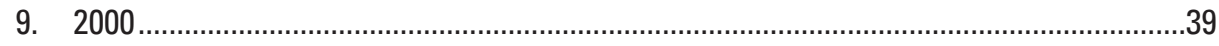

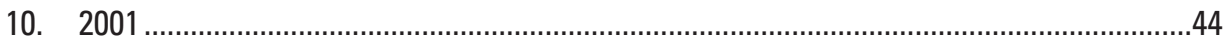

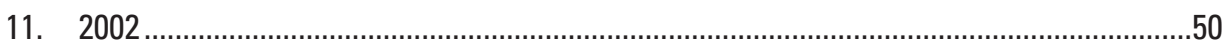

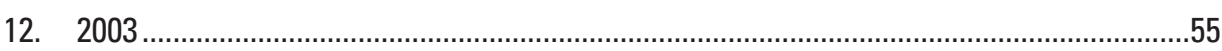

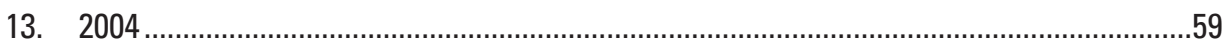

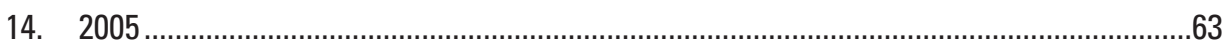

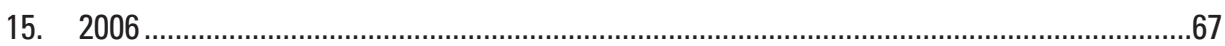

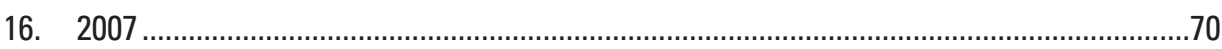

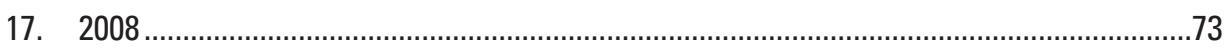

18. Quality-assurance data: selected chemical concentrations in blank samples for 1993-2008. .76

19. Quality-assurance data: relative percent differences in concentrations between environmental and replicate samples for 1993-2008...... 


\section{Conversion Factors and Datums}

\begin{tabular}{|c|c|c|}
\hline Multiply & By & To obtain \\
\hline \multicolumn{3}{|c|}{ Length } \\
\hline centimeter $(\mathrm{cm})$ & 0.3937 & inch (in.) \\
\hline meter $(\mathrm{m})$ & 3.281 & foot $(\mathrm{ft})$ \\
\hline kilometer $(\mathrm{km})$ & 0.6214 & mile (mi) \\
\hline \multicolumn{3}{|c|}{ Volume } \\
\hline liter $(\mathrm{L})$ & 33.82 & ounce, fluid (fl. oz) \\
\hline \multicolumn{3}{|c|}{ Mass } \\
\hline nanogram (ng) & $3.53 \times 10^{-11}$ & ounce (oz.) \\
\hline
\end{tabular}

$\mu \mathrm{S} / \mathrm{cm}$, microsiemens per centimeter

$\mu e q / L$, microequivalents per liter

To convert microequivalents per liter for major ions, divide microequivalents by factors indicated for each ion:

\begin{tabular}{cc}
\hline To obtain milligrams per liter for & divide by \\
\hline $\mathrm{H}^{+}$ & 1,000 \\
$\mathrm{Ca}^{2+}$ & 49.90 \\
$\mathrm{Mg}^{2+}$ & 82.26 \\
$\mathrm{~K}^{+}$ & 25.57 \\
$\mathrm{Na}^{+}$ & 43.50 \\
$\mathrm{NH}_{4}^{+}$ & 55.44 \\
$\mathrm{SO}_{4}^{2-}$ & 20.83 \\
$\mathrm{NO}_{3}^{-}$ & 16.13 \\
$\mathrm{Cl}^{-}$ & 28.21 \\
\hline
\end{tabular}

Temperature in degrees Celsius $\left({ }^{\circ} \mathrm{C}\right)$ may be converted to degrees Fahrenheit $\left({ }^{\circ} \mathrm{F}\right)$ as follows:

$$
{ }^{\circ} \mathrm{F}=\left(1.8 x^{\circ} \mathrm{C}\right)+32
$$

Vertical coordinate information is referenced to the North American Vertical Datum of 1988 (NAVD 88).

Horizontal coordinate information is referenced to the North American Datum of 1983 (NAD 83). Altitude, as used in this report, refers to distance above the vertical datum. 


\title{
Rocky Mountain Snowpack Physical and Chemical Data for Selected Sites, 1993-2008
}

\author{
By George P. Ingersoll, M. Alisa Mast, Donald H. Campbell, David W. Clow, Leora Nanus, and John T. Turk
}

\section{Abstract}

The Rocky Mountain Snowpack program established a network of snowpack-sampling sites in the Rocky Mountain region from New Mexico to Montana to monitor the chemical content of snow to help in the understanding of the effects of atmospheric deposition to this region. The U.S. Geological Survey, in cooperation with the National Park Service, the USDA Forest Service, Teton County in Wyoming, Rio Blanco County in Colorado, Pitkin County in Colorado, and others, collected and analyzed snowpack samples annually for 48 or more sites in the Rocky Mountain region during 1993-2008. Forty-eight of the 162 snow-sampling sites have been sampled annually since 1993. Data include acid-neutralization capacity, specific conductance, $\mathrm{pH}$, hydrogen ion concentrations, dissolved concentrations of major constituents (calcium, magnesium, sodium, potassium, ammonium, chloride, sulfate, and nitrate), dissolved organic carbon concentrations, snow/ water equivalent, snow depth, stable sulfur isotope ratios, total mercury concentrations (beginning in 2001), and ionic charge balance. Quality-assurance data for field and laboratory blanks and field replicates for individual years (1993-2008) also are included.

\section{Introduction}

The U.S. Geological Survey, in cooperation with the National Park Service, the USDA Forest Service, Teton County in Wyoming, Rio Blanco County in Colorado, Pitkin County in Colorado, and others, has been collecting and analyzing snowpack samples from a network of 162 sites in the Rocky Mountain region. Because snowmelt supplies most of the freshwater in mountain lakes, streams, and wetlands in the Rocky Mountain region, monitoring the chemical content of snow is critical to understanding the effects of atmospheric deposition to these systems. This cooperative program has developed methods for measuring physical and chemical properties of seasonal snowpacks for the purpose of deter- mining atmospheric deposition in many areas of the region where no other monitoring has been done. Forty-eight of these snowpack sites have been sampled annually since 1993. More details about the history of the program are available in Ingersoll and others (2002).

\section{Purpose and Scope}

The purpose of this report is to publish the 1993-2008 Rocky Mountain snowpack physical and chemical data in an easily accessible document. This report contains site information for 162 snowpack sites that were sampled and analyzed from 1993 through 2008 and individual data tables for each year. The data tables include acid-neutralization capacity, specific conductance, $\mathrm{pH}$, hydrogen ion concentrations, dissolved concentrations of major constituents (calcium, magnesium, sodium, potassium, ammonium, chloride, sulfate, and nitrate), dissolved organic carbon (DOC) concentrations, snow/ water equivalent, snow depth, stable sulfur isotope ratios, total mercury concentrations (beginning in 2001), and ionic charge balance.

\section{Study Area}

To identify regional emission signals in atmospheric deposition of nitrogen, sulfur, and mercury, the snowpacksampling sites were selected primarily along the Continental Divide in Montana, Idaho, Wyoming, Utah, Colorado, and New Mexico, in areas that are exposed to limited atmospheric emissions from local residential, commercial, or industrial activities (fig. 1). Snow-sampling sites were located at least $30 \mathrm{~m}$ away from plowed roadways to minimize contamination from vehicular traffic. Colorado and New Mexico sites range in elevation from about 2,500 to 3,600 m; sites in Idaho, Utah, Wyoming, and Montana typically are lower at about 1,100 to 3,200 m (table 1). 


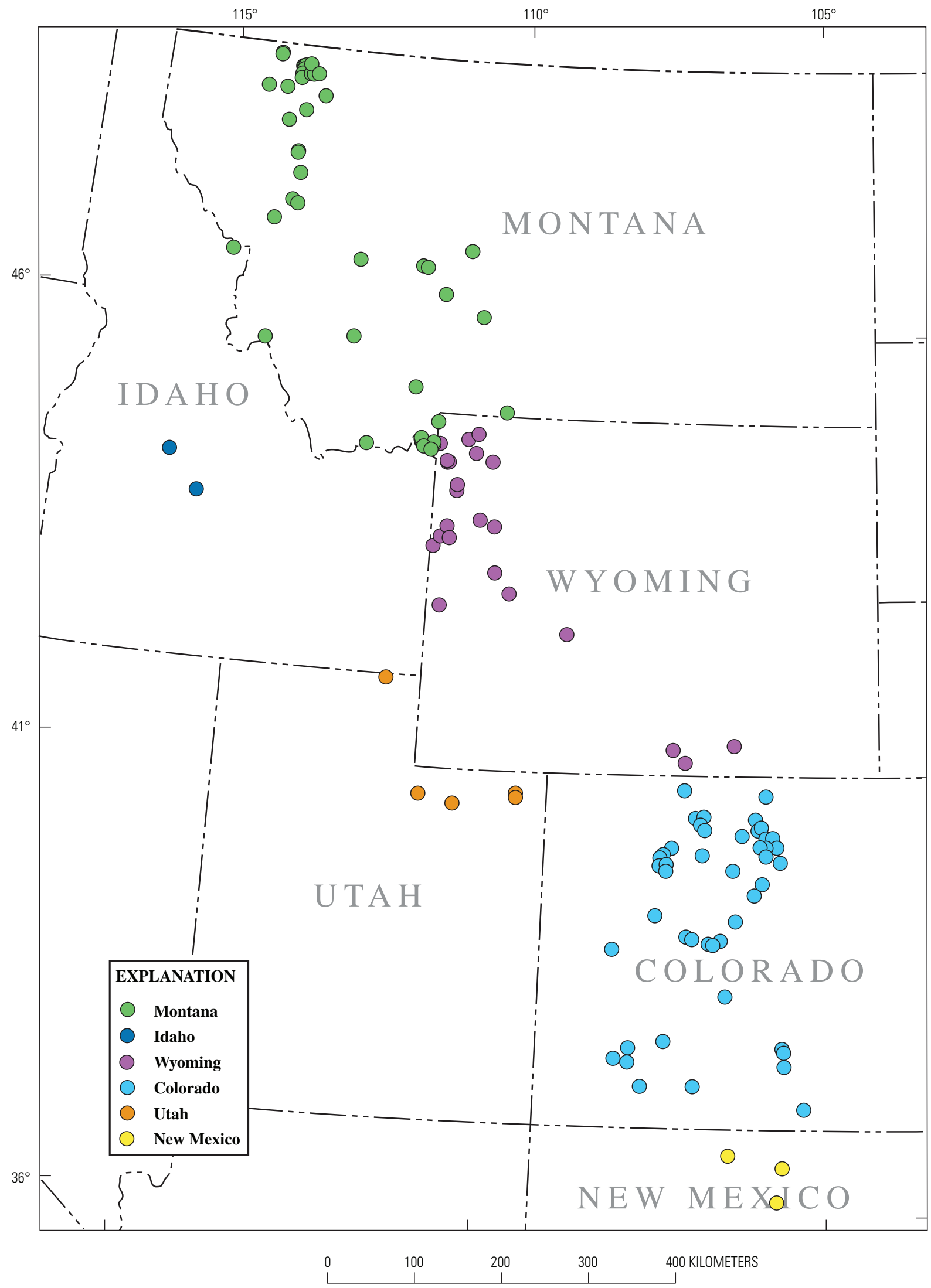

Figure 1. Study area and snowpack-sampling sites in Rocky Mountain region of the United States. 
Table 1. Station ID and snow-sampling-site location information.

[dd, decimal degrees; m, meters above the North American Vertical Datum of 1988; FIPS, Federal Information Processing Standards; YNP, Yellowstone National Park; NP, National park; NADP, National Atmospheric Deposition Program].

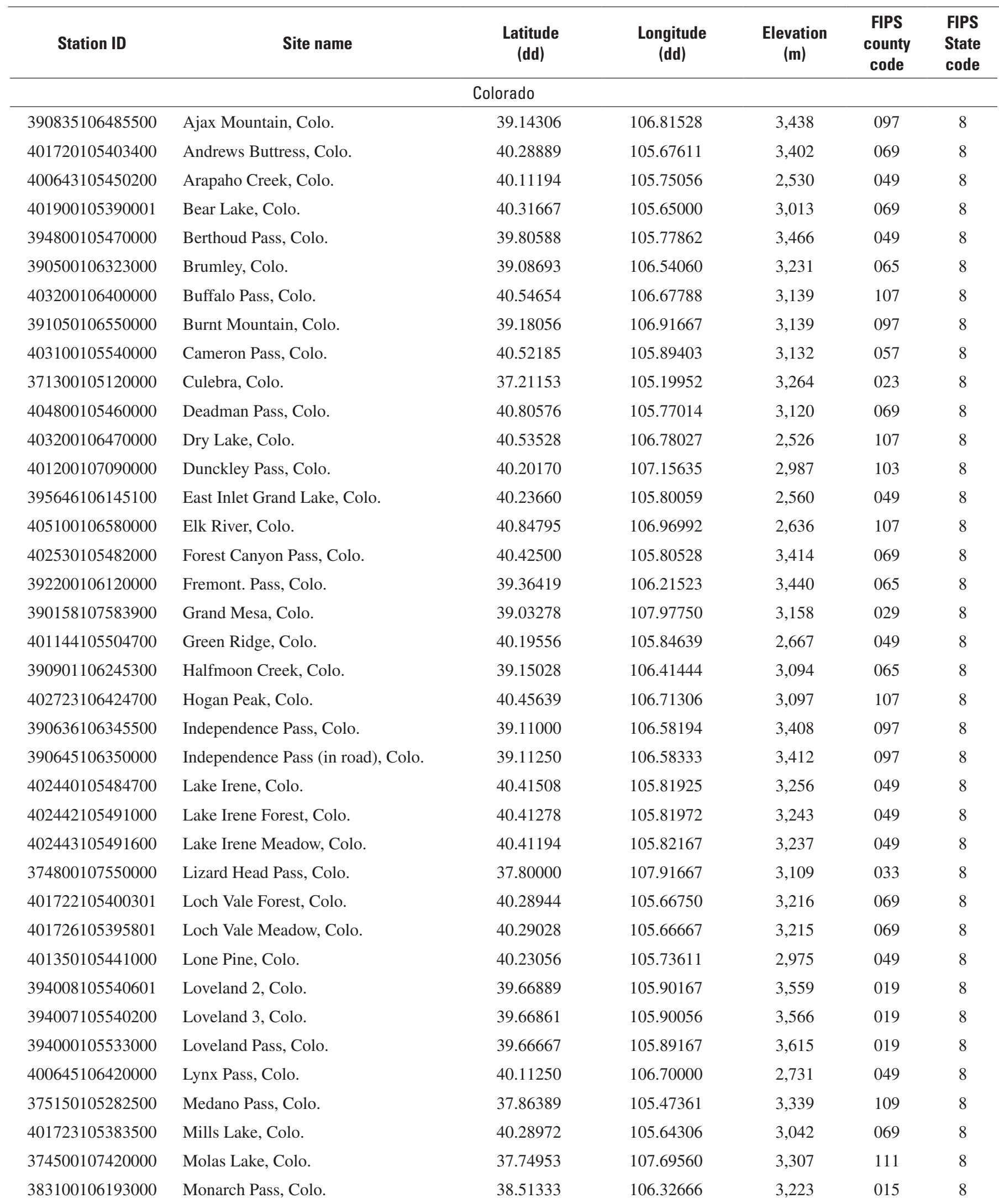


Table 1. Station ID and snow-sampling-site location information.-Continued

[dd, decimal degrees; m, meters above the North American Vertical Datum of 1988; FIPS, Federal Information Processing Standards; YNP, Yellowstone National Park; NP, National park; NADP, National Atmospheric Deposition Program].

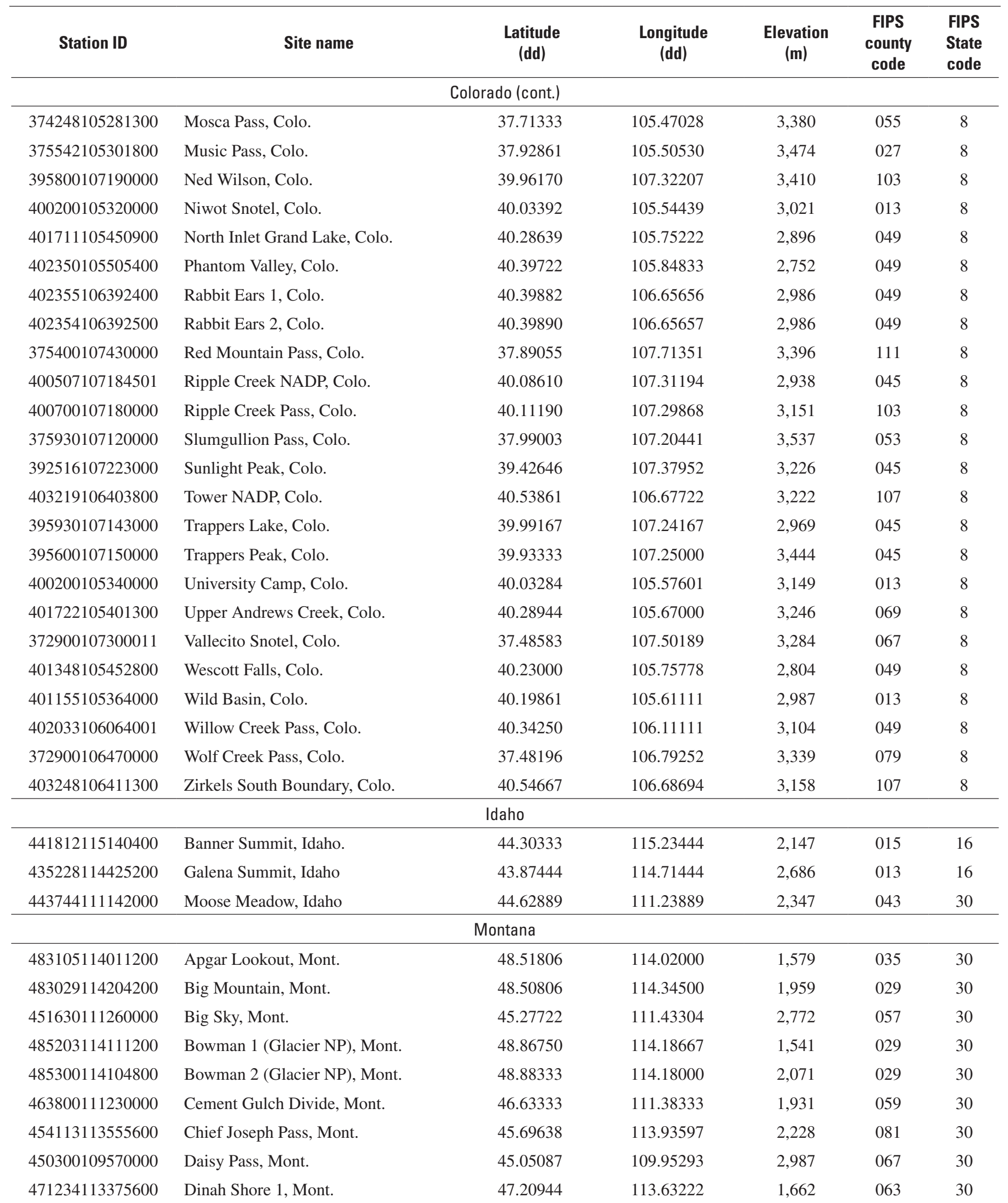


Table 1. Station ID and snow-sampling-site location information.-Continued

[dd, decimal degrees; m, meters above the North American Vertical Datum of 1988; FIPS, Federal Information Processing Standards; YNP, Yellowstone National Park; NP, National park; NADP, National Atmospheric Deposition Program].

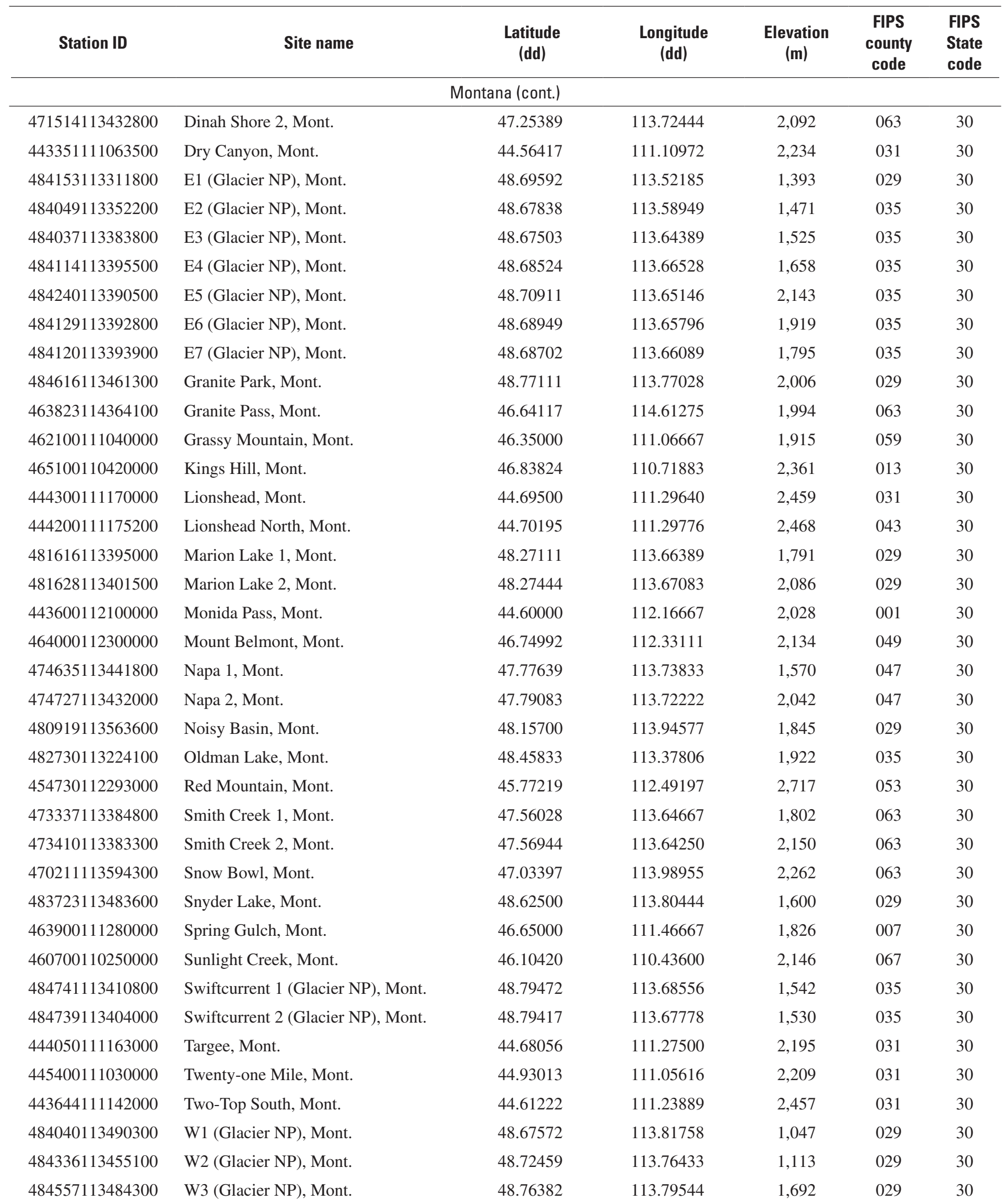


Table 1. Station ID and snow-sampling-site location information.-Continued

[dd, decimal degrees; m, meters above the North American Vertical Datum of 1988; FIPS, Federal Information Processing Standards; YNP, Yellowstone National Park; NP, National park; NADP, National Atmospheric Deposition Program].

\begin{tabular}{|c|c|c|c|c|c|c|}
\hline Station ID & Site name & $\begin{array}{l}\text { Latitude } \\
\text { (dd) }\end{array}$ & $\begin{array}{l}\text { Longitude } \\
\text { (dd) }\end{array}$ & $\begin{array}{l}\text { Elevation } \\
\text { (m) }\end{array}$ & $\begin{array}{c}\text { FIPS } \\
\text { county } \\
\text { code }\end{array}$ & $\begin{array}{l}\text { FIPS } \\
\text { State } \\
\text { code }\end{array}$ \\
\hline 484443113464400 & W5 (Glacier NP), Mont. & 48.74330 & 113.77882 & 1,112 & 029 & 30 \\
\hline 484633113460000 & W6 (Glacier NP), Mont. & 48.77396 & 113.76658 & 2,130 & 029 & 30 \\
\hline 484644113454900 & W7 (Glacier NP), Mont. & 48.77697 & 113.76363 & 2,192 & 029 & 30 \\
\hline 484553113482100 & W10 (Glacier NP), Mont. & 48.76261 & 113.80594 & 1,479 & 029 & 30 \\
\hline 444000111060000 & West Yellowstone, Mont. & 44.65845 & 111.09061 & 2,035 & 031 & 30 \\
\hline 443900111050000 & West Yellowstone (in road), Mont. & 44.65716 & 111.09086 & 2,032 & 031 & 30 \\
\hline 444030111060000 & West Yellowstone 1000m, Mont. & 44.66700 & 111.08500 & 2,027 & 031 & 30 \\
\hline 364300106160000 & Hopewell, N.Mex. & 36.70930 & 106.24763 & 3,036 & 039 & 35 \\
\hline 363429105273000 & Taos Ski Valley, N.Mex. & 36.57286 & 105.44525 & 3,320 & 055 & 35 \\
\hline \multicolumn{7}{|c|}{ Utah } \\
\hline 415803111331900 & Beaver Mountain, Utah & 41.96750 & 111.55528 & 2,577 & 033 & 49 \\
\hline 404456109301800 & Grizzly Ridge, Utah & 40.74886 & 109.50513 & 2,914 & 047 & 49 \\
\hline 403544110260200 & Lake Fork, Utah & 40.59556 & 110.43389 & 3,094 & 013 & 49 \\
\hline 404227109294600 & Little Brush Creek, Utah & 40.70750 & 109.49611 & 2,469 & 047 & 49 \\
\hline 404052110570700 & Trial Lake, Utah & 40.68111 & 110.95194 & 2,999 & 043 & 49 \\
\hline 411800107100000 & Divide Peak, Wyo. & 41.30472 & 107.15972 & 2,634 & 007 & 56 \\
\hline 430000109450000 & Elkhart Park, Wyo. & 43.00265 & 109.75698 & 2,865 & 035 & 56 \\
\hline 434900110160000 & Four Mile Meadow, Wyo. & 43.82363 & 110.26472 & 2,406 & 039 & 56 \\
\hline 434326110465900 & Garnet Canyon, Wyo. & 43.71003 & 110.75360 & 2,174 & 039 & 56 \\
\hline 431322109592700 & Gypsum Creek, Wyo. & 43.22925 & 109.99613 & 2,516 & 035 & 56 \\
\hline 433600110440000 & Jackson Hole Airport, Wyo. & 43.60490 & 110.73400 & 1,965 & 039 & 56 \\
\hline 443357110235900 & Lake, Wyo. & 44.56674 & 110.40031 & 2,426 & 039 & 56 \\
\hline 441300110400000 & Lewis Lake Divide, Wyo. & 44.19934 & 110.66045 & 2,363 & 039 & 56 \\
\hline 441300110395900 & Lewis Lake Divide (in road), Wyo. & 44.19948 & 110.65941 & 2,357 & 039 & 56 \\
\hline 410900107580000 & Old Battle, Wyo. & 41.15464 & 106.97577 & 3,024 & 007 & 56 \\
\hline 442640110503300 & Old Faithful (in road), Wyo. & 44.45586 & 110.83376 & 2,250 & 029 & 56 \\
\hline 442727110505500 & Old Faithful 1000m, Wyo. & 44.44444 & 110.84250 & 2,302 & 039 & 56 \\
\hline 442725110492799 & Old Faithful Crew Corrals, Wyo. & 44.45750 & 110.84861 & 2,256 & 039 & 56 \\
\hline 442720110500300 & Old Faithful East Lot, Wyo. & 44.45694 & 110.82444 & 2,237 & 039 & 56 \\
\hline
\end{tabular}


Table 1. Station ID and snow-sampling-site location information.-Continued

[dd, decimal degrees; m, meters above the North American Vertical Datum of 1988; FIPS, Federal Information Processing Standards; YNP, Yellowstone National Park; NP, National park; NADP, National Atmospheric Deposition Program].

\begin{tabular}{|c|c|c|c|c|c|c|}
\hline Station ID & Site name & $\begin{array}{l}\text { Latitude } \\
\text { (dd) }\end{array}$ & $\begin{array}{l}\text { Longitude } \\
\text { (dd) }\end{array}$ & $\begin{array}{l}\text { Elevation } \\
(\mathrm{m})\end{array}$ & $\begin{array}{c}\text { FIPS } \\
\text { county } \\
\text { code }\end{array}$ & $\begin{array}{l}\text { FIPS } \\
\text { State } \\
\text { code }\end{array}$ \\
\hline 442721110500300 & Old Faithful Fire Road, Wyo. & 44.45603 & 110.83458 & 2,246 & 039 & 56 \\
\hline 442732110494600 & Old Faithful West Lot, Wyo. & 44.45827 & 110.82993 & 2,240 & 039 & 56 \\
\hline 440809110395900 & South Entrance YNP (in road), Wyo. & 44.13668 & 110.66759 & 2,100 & 039 & 56 \\
\hline 423420108503200 & South Pass, Wyo. & 42.57230 & 108.84272 & 2,755 & 013 & 56 \\
\hline 442900110090000 & Sylvan Lake, Wyo. & 44.47436 & 110.15481 & 2,566 & 029 & 56 \\
\hline 442900110090100 & Sylvan Lake (in road), Wyo. & 44.47364 & 110.15485 & 2,572 & 029 & 56 \\
\hline 444730110230000 & Tower Falls, Wyo. & 44.88518 & 110.38391 & 2,008 & 029 & 56 \\
\hline 443914110593000 & West Yellowstone 8km, Wyo. & 44.65390 & 110.99170 & 2,066 & 039 & 56 \\
\hline 443915110593200 & West Yellowstone 8km (in road), Wyo. & 44.65410 & 110.99210 & 2,065 & 039 & 56 \\
\hline 424912110500700 & Willow Creek, Wyo. & 42.82000 & 110.83528 & 2,414 & 023 & 56 \\
\hline
\end{tabular}




\section{Sampling Methods and Analyses}

Snowpacks were sampled annually from late February through early April according to field methods described in Ingersoll and others (2005). Snow depth was measured at all sites and snow/water equivalent (SWE) was measured at selected sites during the period. Snow samples from sites with complete chemical analyses of the majority of annual snowpack years were selected for this report. In addition to the 48 original long-term sites, snowpack physical and chemical data were collected at another 114 sites one or more times to address short-term interests. Discussion of the results of the monitoring program is available in other reports and publications (Ingersoll and others, 2002; Mast and others, 2005).

Analytical laboratory methods and quality-assurance procedures for analyses of major-ion and mercury concentrations and stable sulfur isotope ratios are described in Mast and others (2001), Turk and others (2001), and Ingersoll and others (2005). Laboratory reporting limits (LRL) are based on long-term method detection levels (LT-MDL) and are calculated as two times the LT-MDL (for details see Childress and others, 1999). Reporting limits referred to in tables 2-17 are $1.0 \mu \mathrm{S} / \mathrm{cm}$ for specific conductance, 0.4 to $3.1 \mu \mathrm{eq} / \mathrm{L}$ for major dissolved constituents and nutrients (calcium, 3.1; magnesium, 1.7; sodium, 1.0; potassium, 0.4; ammonium, 1.0; chloride, 1.0; sulfate, 0.6; nitrate, 0.4$), 0.4$ milligram per liter $(\mathrm{mg} / \mathrm{L})$ for dissolved organic carbon (DOC), and 0.4 nanogram per liter (ng/L) for total (whole-water) mercury (beginning in 2001; no total-mercury data are available for the previous years). Censored values (preceded by "<") in this report reflect nondetection based on the LRLs. Ammonium and nitrate concentrations are reported as $\mathrm{NH}_{4}{ }^{+}$and $\mathrm{NO}_{3}^{-}$, respectively (and not as $\mathrm{N}$ ); sulfate concentrations are reported as $\mathrm{SO}_{4}{ }^{2-}$ (and not as $\mathrm{S})$. Negative acid neutralization capacity (ANC) values shown in this report reflect the absence of bicarbonate ion at the typically low $\mathrm{pH}$ levels, although for many samples positive ANC was detected.

Quality-assurance data for field and laboratory blanks and field replicates for individual years (1993-2008) were collected and analyzed (tables 18-19). High-purity deionized water was used for all blanks. Quality-assurance data for field and laboratory blanks show little contamination and indicate few outliers. Four percent of the 370 constituent analyses of laboratory blanks analyzed for major ions and 6 percent of the 462 constituent analyses of field blanks analyzed for major ions showed concentrations above the detection limit. Sulfate, nitrate, DOC, and mercury showed the largest percentages of detections both in laboratory blank samples $(9,7,13$, and 36 percent, respectively) and in field blank samples (10, 17, 27 , and 35 percent, respectively). Trace levels of mercury were detected in about 35 percent of the 26 field blanks that were analyzed for total mercury. For field blank samples with detectable concentrations of mercury, the average concentration was $0.7 \mathrm{ng} / \mathrm{L}$. Additional information including interlaboratory comparisons of USGS standard reference samples is available at http://bqs.usgs.gov/srs.
As a quality-control measure, ionic charge balances of each major-ion analysis were calculated by dividing the sum of cations (hydrogen ion, calcium, magnesium, sodium, potassium, and ammonium) minus the sum of anions (ANC [greater than 0.0], chloride, nitrate, and sulfate) by the total cations and anions in solution. Censored values were not included in ionic balances. In general, ionic balances of results of chemical analyses for many samples included in this report had a positive bias believed to be due to organic acids that were not analyzed (Turk and others, 2001). This positive bias indicating an excess of cations also has been found in other precipitation work in the Western United States (National Atmospheric Deposition Program, 2001, 2005). In this report the same criteria were used to identify the maximum acceptable ion percent difference as applied by the National Atmospheric Deposition Program (2006). Suspect ionic balances were identified by the following three criteria: (1) for total anions plus cations less than $50 \mu \mathrm{eq} / \mathrm{L}$, the ionic balance exceeds plus or minus 60 percent; (2) for total anions plus cations greater than or equal to $50 \mu \mathrm{eq} / \mathrm{L}$ and less than $100 \mu \mathrm{eq} / \mathrm{L}$, the ionic balance exceeds plus or minus 30 percent; and (3) for total anions plus cations greater than or equal to $100 \mu \mathrm{eq} / \mathrm{L}$, the ionic balance exceeds plus or minus 15 percent. Twelve analyses ( 0.8 percent $)$ met one of these criteria but are retained in this database for user evaluation, and those ionic balances are flagged by parentheses in the data tables.

To quantify comparisons of concentrations of selected major constituents between environmental samples and replicate samples, relative percent differences (RPD) were calculated. The RPD (expressed as a percentage in this report) is the absolute value of the difference of environmental sample concentration (E) minus the replicate sample concentration (R), divided by the average of the environmental sample concentration and the replicate sample concentration, and multiplied by $100(\mid \mathrm{E}-\mathrm{R} / /[(\mathrm{E}+\mathrm{R}) / 2]) \times 100$. It is important to realize that, as dilute concentrations approach detection limits, relative percent differences between environmental sample concentrations and replicate sample concentrations appear to be substantial whereas absolute differences are small. For example, the RPD for two mercury samples of 1.3 and 2.0 nanograms per liter is 42.4 percent. The median RPD for the 10 constituents ranged from 4 to 21 percent (table 19). Potassium, DOC, and mercury showed the greatest variation in $\operatorname{RPD}(21,19$, and 20 percent), indicating concentrations near the detection limit for potassium and DOC and greater variability in mercury concentrations. Overall, the replicate samples show good precision.

\section{Snowpack Physical and Chemical Data}

Site information, including location and elevation, is listed in table 1. Physical and chemical data are arranged by individual years, 1993-2008, and are listed in tables 2-17. Quality-assurance data for chemical analyses of blank and replicate snow samples are listed in tables 18 and 19. 
Table 2. Selected chemical and physical data for Rocky Mountain snowpack samples for 1993.

[E, depth-integrated environmental; VWM, volume-weighted mean of two snowpack layers; ANC, acid neutralization capacity; $\mu$ eq/L, microequivalents per liter; $\mu$ S/cm, microsiemens per centimeter; SC, specific conductance at $25^{\circ} \mathrm{C} ; \mathrm{pH}$ values in standard units; $\mathrm{H}$, hydrogen; $\mathrm{Ca}$, calcium; $\mathrm{Mg}$, magnesium; $\mathrm{Na}$, sodium; $\mathrm{K}$, potassium; $\mathrm{NH}_{4}$, ammonium; $\mathrm{Cl}_{\text {, chloride; }} \mathrm{SO}_{4}$, sulfate; $\mathrm{NO}_{3}$, nitrate; $\mathrm{DOC}$, dissolved organic carbon; $\mathrm{mg} / \mathrm{L}$, milligrams per liter; SWE, snow/water equivalent; $\mathrm{cm}$, centimeters; $\delta^{34} \mathrm{~S}$, stable sulfur isotope ratio $\left.{ }^{34} \mathrm{~S} /{ }^{2} \mathrm{~S}\right) ; \mathrm{Hg}$, mercury; ng/L, nanograms per liter; na, not analyzed; <, below reporting limits; $\%$, percent]

\begin{tabular}{|c|c|c|c|c|c|c|c|c|c|c|c|c|c|c|c|c|c|c|c|c|}
\hline Site name & $\begin{array}{l}\text { Sample } \\
\text { date }\end{array}$ & $\begin{array}{l}\text { Sample } \\
\text { type }\end{array}$ & $\begin{array}{c}\text { ANC } \\
(\mu e q / L)\end{array}$ & $\begin{array}{c}\text { SC } \\
(\mu \mathrm{S} / \mathrm{cm})\end{array}$ & pH & 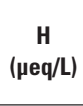 & $\begin{array}{c}\text { Ca } \\
(\mu \mathrm{eq} / \mathrm{L})\end{array}$ & $\begin{array}{c}\mathrm{Mg} \\
(\mu \mathrm{eq} / \mathrm{L})\end{array}$ & $\begin{array}{c}\mathrm{Na} \\
(\mu \mathrm{eq} / \mathrm{L})\end{array}$ & $\begin{array}{c}K \\
(\mu e q / L)\end{array}$ & $\begin{array}{c}\mathrm{NH}_{4} \\
(\mu \mathrm{eq} / \mathrm{L})\end{array}$ & $\begin{array}{c}C l \\
(\mu e q / L)\end{array}$ & $\begin{array}{c}\mathrm{SO}_{4} \\
(\mu \mathrm{eq} / \mathrm{L})\end{array}$ & $\begin{array}{c}\mathrm{NO}_{3} \\
(\mu \mathrm{eq} / \mathrm{L})\end{array}$ & $\begin{array}{l}\text { DOC } \\
\text { (mg/L) }\end{array}$ & $\begin{array}{l}\text { SWE } \\
\text { (cm) }\end{array}$ & $\begin{array}{l}\text { Snow } \\
\text { depth } \\
\text { (cm) }\end{array}$ & $\begin{array}{c}\delta^{34} \mathbf{S} \\
\text { (per mil) }\end{array}$ & $\begin{array}{c}\mathrm{Hg} \\
\text { (ng/L) }\end{array}$ & $\begin{array}{c}\text { Ionic } \\
\text { balance } \\
(\%)\end{array}$ \\
\hline $\begin{array}{l}\text { Berthoud Pass, } \\
\text { Colo. }\end{array}$ & 4/19/1993 & E & na & 5.6 & 5.03 & 9.3 & 3.7 & $<1.7$ & 1.5 & $<0.4$ & 3.2 & 1.1 & 6.3 & 7.9 & 0.4 & 74 & 240 & 4.8 & na & 7.6 \\
\hline $\begin{array}{l}\text { Big Mountain, } \\
\text { Mont. }\end{array}$ & 4/2/1993 & E & -13.3 & 4.6 & 5.11 & 7.8 & $<3.1$ & $<1.7$ & $<1.0$ & $<0.4$ & 3.2 & $<1.0$ & 5.4 & 5.3 & $<0.4$ & 89 & 208 & 5.3 & na & 1.3 \\
\hline Big Sky, Mont. & 4/6/1993 & E & na & 4.2 & 5.35 & 4.5 & 5.1 & $<1.7$ & 4.1 & 0.4 & 5.4 & 1.1 & 6.7 & 7.6 & 0.5 & 38 & 138 & 4.1 & na & 11.7 \\
\hline $\begin{array}{l}\text { Brooklyn Lake, } \\
\text { Wyo. }\end{array}$ & $3 / 22 / 1993$ & E & -14.7 & 5.9 & 5.02 & 9.5 & 3.9 & $<1.7$ & 1.4 & $<0.4$ & 3.6 & 1.1 & 8.8 & 7.5 & 0.4 & 61 & 179 & 6.4 & na & 2.9 \\
\hline Brumley, Colo. & $3 / 29 / 1993$ & $\mathrm{E}$ & -10.3 & 4.1 & 5.19 & 6.5 & 3.5 & $<1.7$ & $<1.0$ & 0.6 & 2.1 & $<1.0$ & 6.3 & 5.6 & $<0.4$ & 38 & 143 & na & na & 3.3 \\
\hline $\begin{array}{l}\text { Buffalo Pass, } \\
\text { Colo. }\end{array}$ & 4/8/1993 & $\mathrm{E}$ & na & 8.1 & 4.80 & 15.8 & $<3.1$ & $<1.7$ & 1.7 & $<0.4$ & 3.9 & $<1.0$ & 11.5 & 8.4 & $<0.4$ & 122 & 325 & 6.2 & na & 3.8 \\
\hline $\begin{array}{l}\text { Cameron Pass, } \\
\text { Colo. }\end{array}$ & 4/20/1993 & E & na & 6.1 & 5.03 & 9.3 & 4.7 & $<1.7$ & 2.3 & 1.1 & 3.9 & 2.0 & 9.0 & 7.3 & 0.5 & 89 & 260 & 4.6 & na & 7.5 \\
\hline Canyon, Wyo. & $3 / 28 / 1993$ & E & -14.9 & 4.5 & 5.14 & 7.2 & $<3.1$ & $<1.7$ & 1.4 & $<0.4$ & 3.2 & 1.1 & 5.0 & 6.0 & $<0.4$ & 28 & 105 & 4.4 & na & -1.1 \\
\hline $\begin{array}{l}\text { Chief Joseph } \\
\text { Pass, Mont. }\end{array}$ & 4/4/1993 & E & -10.1 & 3.2 & 5.34 & 4.6 & $<3.1$ & $<1.7$ & $<1.0$ & $<0.4$ & 3.2 & $<1.0$ & 4.0 & 2.8 & $<0.4$ & 48 & 149 & 4.8 & na & 6.9 \\
\hline $\begin{array}{l}\text { Daisy Pass, } \\
\text { Mont. }\end{array}$ & 4/6/1993 & E & -11.8 & 4.1 & 5.26 & 5.5 & $<3.1$ & $<1.7$ & $<1.0$ & $<0.4$ & 4.3 & $<1.0$ & 6.3 & 4.6 & 0.4 & 29 & 118 & 4.1 & na & -5.3 \\
\hline $\begin{array}{l}\text { Deadman Pass, } \\
\text { Colo. }\end{array}$ & $4 / 20 / 1993$ & E & na & 5.2 & 5.24 & 5.8 & 4.8 & $<1.7$ & 1.0 & 1.1 & 6.4 & $<1.0$ & 8.3 & 8.3 & 0.7 & 46 & 155 & 4.4 & na & 6.9 \\
\hline $\begin{array}{l}\text { Divide Peak, } \\
\text { Wyo. }\end{array}$ & $3 / 23 / 1993$ & E & -22.2 & 9.0 & 4.76 & 17.4 & $<3.1$ & $<1.7$ & $<1.0$ & $<0.4$ & 3.6 & $<1.0$ & 12.1 & 10.8 & $<0.4$ & 33 & 115 & 6.2 & na & -4.4 \\
\hline Dry Lake, Colo. & 4/7/1993 & E & na & 8.6 & 4.81 & 15.5 & 5.6 & $<1.7$ & $<1.0$ & 2.5 & 5.0 & $<1.0$ & 12.3 & 13.8 & 0.5 & 51 & 140 & 6.6 & na & 4.6 \\
\hline $\begin{array}{l}\text { Dunckley Pass, } \\
\text { Colo. }\end{array}$ & 4/7/1993 & E & -12.2 & 5.6 & 5.10 & 7.9 & 5.0 & $<1.7$ & 1.0 & 3.8 & 3.9 & 1.1 & 8.8 & 7.7 & 0.9 & 56 & 190 & 5.8 & na & 10.4 \\
\hline Elk River, Colo. & 4/7/1993 & $\mathrm{E}$ & na & 7.2 & 4.86 & 13.8 & $<3.1$ & $<1.7$ & 1.0 & $<0.4$ & 2.5 & $<1.0$ & 6.9 & 12.0 & $<0.4$ & 48 & 123 & 5.4 & na & -4.3 \\
\hline $\begin{array}{l}\text { Elkhart Park, } \\
\text { Wyo. }\end{array}$ & $3 / 25 / 1993$ & E & -10.7 & 3.9 & 5.42 & 3.8 & $<3.1$ & $<1.7$ & $<1.0$ & 0.5 & 8.9 & $<1.0$ & 6.0 & 5.4 & $<0.4$ & 36 & 135 & 3.9 & na & 7.2 \\
\hline $\begin{array}{l}\text { Four Mile } \\
\text { Meadow, } \\
\text { Wyo. }\end{array}$ & $3 / 26 / 1993$ & E & 0.4 & 3.5 & 5.39 & 4.1 & 3.7 & $<1.7$ & 1.1 & 0.5 & 3.2 & $<1.0$ & 5.0 & 5.4 & 0.4 & 23 & 97 & 5.7 & na & 7.8 \\
\hline $\begin{array}{l}\text { Fremont Pass, } \\
\text { Colo. }\end{array}$ & 4/18/1993 & E & na & 6.6 & 5.25 & 5.6 & 5.6 & $<1.7$ & 1.1 & 0.8 & 3.2 & $<1.0$ & 5.6 & 6.4 & 0.7 & 46 & 150 & 3.7 & na & 15.6 \\
\hline $\begin{array}{l}\text { Gallegos Peak, } \\
\text { N. Mex. }\end{array}$ & 3/13/1993 & $\mathrm{E}$ & -11.3 & 4.6 & 5.21 & 6.2 & 7.3 & $<1.7$ & $<1.0$ & 2.7 & 1.8 & $<1.0$ & 9.0 & 4.5 & 0.9 & 41 & 134 & 3.2 & na & 14.1 \\
\hline $\begin{array}{l}\text { Garnet Canyon, } \\
\text { Wyo. }\end{array}$ & 4/15/1993 & E & na & 4.6 & 5.15 & 7.1 & 3.7 & 2.5 & 1.9 & $<0.4$ & 3.9 & $<1.0$ & 7.5 & 5.0 & $<0.4$ & 99 & 265 & 5.4 & na & 20.9 \\
\hline
\end{tabular}


Table 2. Selected chemical and physical data for Rocky Mountain snowpack samples for 1993.—Continued

[E, depth-integrated environmental; VWM, volume-weighted mean of two snowpack layers; ANC, acid neutralization capacity; ueq/L, microequivalents per liter; $\mu \mathrm{S} / \mathrm{cm}$, microsiemens per centimeter; SC, specific conductance at $25^{\circ} \mathrm{C} ; \mathrm{pH}$ values in standard units; $\mathrm{H}$, hydrogen; $\mathrm{Ca}$, calcium; $\mathrm{Mg}$, magnesium; $\mathrm{Na}$, sodium; $\mathrm{K}$, potassium; $\mathrm{NH}_{4}$, ammonium; $\mathrm{Cl}_{\text {, chloride; }} \mathrm{SO}_{4}$, sulfate; $\mathrm{NO}$, nitrate; $\mathrm{DOC}$, dissolved organic carbon; mg/L, milligrams per liter; SWE, snow/water equivalent; cm, centimeters; $\delta^{34} \mathrm{~S}$, stable sulfur isotope ratio $\left.{ }^{34} \mathrm{~S} /{ }^{2} \mathrm{~S}\right) ; \mathrm{Hg}$, mercury; ng/L, nanograms per liter; na, not analyzed; <, below reporting limits; $\%$, percent]

\begin{tabular}{|c|c|c|c|c|c|c|c|c|c|c|c|c|c|c|c|c|c|c|c|c|}
\hline Site name & $\begin{array}{c}\text { Sample } \\
\text { date }\end{array}$ & $\begin{array}{c}\text { Sample } \\
\text { type }\end{array}$ & $\begin{array}{c}\text { ANC } \\
\text { ( } \mu \mathrm{eq} / \mathrm{L})\end{array}$ & $\underset{(\mu \mathrm{S} / \mathrm{cm})}{\mathrm{SC}}$ & $\mathrm{pH}$ & $\begin{array}{c}H \\
\text { ( } \mu \mathrm{eq} / \mathrm{L} \text { ) }\end{array}$ & $\begin{array}{c}\mathrm{Ca} \\
\text { ( } \mu \mathrm{eq} / \mathrm{L})\end{array}$ & $\begin{array}{c}\mathrm{Mg} \\
(\mu \mathrm{eq} / \mathrm{L})\end{array}$ & $\begin{array}{c}\mathrm{Na} \\
(\mu \mathrm{eq} / \mathrm{L})\end{array}$ & $\begin{array}{c}K \\
\text { ( } \mu \mathrm{eq} / \mathrm{L} \text { ) }\end{array}$ & $\begin{array}{c}\mathrm{NH}_{4} \\
(\mu \mathrm{eq} / \mathrm{L})\end{array}$ & $\underset{(\mu \mathrm{eq} / \mathrm{L})}{\mathrm{Cl}}$ & $\underset{\text { (peq/L) }}{\mathrm{SO}_{4}}$ & $\begin{array}{c}\mathrm{NO}_{3} \\
(\mu \mathrm{eq} / \mathrm{L})\end{array}$ & $\begin{array}{c}\text { DOC } \\
\text { (mg/L) }\end{array}$ & $\begin{array}{l}\text { SWE } \\
\text { (cm) }\end{array}$ & $\begin{array}{l}\text { Snow } \\
\text { depth } \\
\text { (cm) }\end{array}$ & $\begin{array}{c}\delta^{34} \mathbf{S} \\
\text { (per mil) }\end{array}$ & $\underset{(\mathrm{ng} / \mathrm{L})}{\mathrm{Hg}}$ & $\begin{array}{c}\text { Ionic } \\
\text { balance } \\
(\%)\end{array}$ \\
\hline $\begin{array}{l}\text { Grand Mesa, } \\
\text { Colo. }\end{array}$ & $3 / 21 / 1993$ & $\mathrm{E}$ & -16.8 & 5.4 & 5.04 & 9.1 & $<3.1$ & $<1.7$ & 1.0 & 0.4 & 3.6 & $<1.0$ & 9.6 & 5.8 & $<0.4$ & 84 & 251 & 4.6 & na & -4.2 \\
\hline $\begin{array}{l}\text { Granite Pass, } \\
\text { Mont. }\end{array}$ & 4/3/1993 & E & -4.0 & 3.0 & 5.30 & 5.0 & $<3.1$ & $<1.7$ & $<1.0$ & $<0.4$ & 1.8 & $<1.0$ & 3.3 & 2.2 & $<0.4$ & 56 & 183 & 5.2 & na & 10.4 \\
\hline $\begin{array}{l}\text { Gypsum Creek, } \\
\text { Wyo. }\end{array}$ & $3 / 25 / 1993$ & E & -14.5 & 5.3 & 4.97 & 10.7 & $<3.1$ & $<1.7$ & 1.3 & $<0.4$ & 2.5 & $<1.0$ & 5.0 & 7.1 & $<0.4$ & 31 & 96 & 5.8 & na & 9.3 \\
\hline $\begin{array}{l}\text { Hopewell, } \\
\text { N. Mex. }\end{array}$ & $3 / 14 / 1993$ & E & -16.8 & 6.4 & 4.94 & 11.5 & $<3.1$ & $<1.7$ & $<1.0$ & $<0.4$ & 3.2 & 1.1 & 10.2 & 6.8 & $<0.4$ & 74 & 196 & 3.6 & na & -10.3 \\
\hline $\begin{array}{l}\text { Kings Hill, } \\
\text { Mont. }\end{array}$ & $3 / 31 / 1993$ & E & -10.7 & 5.0 & 5.16 & 6.9 & 5.6 & $<1.7$ & 1.6 & 0.6 & 7.1 & $<1.0$ & 9.2 & 6.4 & 0.9 & 48 & 138 & 7.5 & na & 16.8 \\
\hline $\begin{array}{c}\text { Lake Irene, } \\
\text { Colo. }\end{array}$ & 4/7/1993 & E & -14.0 & 5.7 & 4.99 & 10.2 & $<3.1$ & $<1.7$ & 1.6 & $<0.4$ & 2.5 & $<1.0$ & 6.5 & 5.3 & $<0.4$ & na & 196 & 5.2 & na & 9.8 \\
\hline $\begin{array}{l}\text { Lewis Lake } \\
\text { Divide, Wyo. }\end{array}$ & $3 / 27 / 1993$ & E & -8.9 & 4.3 & 5.21 & 6.2 & $<3.1$ & $<1.7$ & 1.7 & $<0.4$ & 5.7 & 1.1 & 6.3 & 5.4 & $<0.4$ & 81 & 215 & 4.5 & na & 2.8 \\
\hline $\begin{array}{l}\text { Lionshead, } \\
\text { Mont. }\end{array}$ & 3/30/1993 & E & -15.8 & 7.9 & 5.08 & 8.3 & 5.6 & $<1.7$ & $<1.0$ & 2.9 & 19.6 & 3.1 & 16.5 & 12.5 & 1.0 & 53 & 185 & 4.6 & na & 6.5 \\
\hline $\begin{array}{l}\text { Loch Vale } \\
\text { Meadow, } \\
\text { Colo. }\end{array}$ & $4 / 10 / 1993$ & E & na & 6.1 & 4.96 & 11.0 & $<3.1$ & $<1.7$ & 2.2 & $<0.4$ & 3.9 & $<1.0$ & 7.7 & 7.4 & $<0.4$ & 71 & 199 & 4.5 & na & 6.0 \\
\hline $\begin{array}{l}\text { Loveland Pass, } \\
\text { Colo. }\end{array}$ & $4 / 18 / 1993$ & E & na & 5.2 & 5.07 & 8.5 & 3.7 & $<1.7$ & 1.5 & $<0.4$ & 2.5 & 1.4 & 6.3 & 7.6 & $<0.4$ & 58 & 190 & 4.3 & na & 3.2 \\
\hline $\begin{array}{l}\text { Lynx Pass, } \\
\text { Colo. }\end{array}$ & $3 / 18 / 1993$ & E & -20.2 & 6.8 & 4.88 & 13.2 & $<3.1$ & $<1.7$ & $<1.0$ & $<0.4$ & 2.1 & $<1.0$ & 7.1 & 9.5 & $<0.4$ & 41 & 139 & 5.8 & na & -4.0 \\
\hline $\begin{array}{l}\text { Molas Lake, } \\
\text { Colo. }\end{array}$ & $3 / 31 / 1993$ & E & -13.3 & 4.5 & 5.13 & 7.4 & $<3.1$ & $<1.7$ & 1.3 & $<0.4$ & 1.4 & $<1.0$ & 6.3 & 5.0 & $<0.4$ & 56 & 162 & 2.8 & na & -5.4 \\
\hline $\begin{array}{l}\text { Monarch Pass, } \\
\text { Colo. }\end{array}$ & $3 / 30 / 1993$ & E & -13.8 & 4.8 & 5.22 & 6.0 & 5.6 & $<1.7$ & $<1.0$ & $<0.4$ & 2.9 & $<1.0$ & 6.3 & 7.0 & $<0.4$ & 43 & 135 & 3.7 & na & 4.3 \\
\hline $\begin{array}{l}\text { Ned Wilson, } \\
\text { Colo. }\end{array}$ & 4/8/1993 & VWM & -15.4 & 5.1 & 5.14 & 7.2 & $<3.1$ & $<1.7$ & $<1.0$ & $<0.4$ & 3.2 & 1.8 & 6.2 & 6.6 & $<0.4$ & 95 & 287 & 5.8 & na & -16.5 \\
\hline $\begin{array}{l}\text { Noisy Basin, } \\
\text { Mont. }\end{array}$ & 4/1/1993 & E & -14.9 & 4.8 & 5.11 & 7.8 & $<3.1$ & $<1.7$ & $<1.0$ & $<0.4$ & 4.6 & $<1.0$ & 6.5 & 5.6 & 0.4 & 86 & 260 & 4.9 & na & 1.4 \\
\hline $\begin{array}{c}\text { Old Battle, } \\
\text { Wyo. }\end{array}$ & $3 / 23 / 1993$ & E & na & 6.4 & 4.96 & 11.0 & $<3.1$ & $<1.7$ & 1.0 & na & 4.3 & $<1.0$ & 10.2 & 7.8 & 0.4 & 69 & 223 & 6.0 & na & -4.9 \\
\hline $\begin{array}{l}\text { Phantom Valley, } \\
\text { Colo. }\end{array}$ & 4/6/1993 & E & na & 7.0 & 4.91 & 12.3 & $<3.1$ & $<1.7$ & 1.2 & 0.7 & 4.3 & $<1.0$ & 9.4 & 9.0 & 0.8 & na & 101 & 5.0 & na & 0.4 \\
\hline $\begin{array}{l}\text { Rabbit Ears 1, } \\
\text { Colo. }\end{array}$ & 4/6/1993 & E & na & 8.3 & 4.79 & 16.2 & $<3.1$ & $<1.7$ & 1.1 & $<0.4$ & 4.6 & $<1.0$ & 11.3 & 10.5 & $<0.4$ & 101 & 293 & 6.0 & na & 0.4 \\
\hline
\end{tabular}


Table 2. Selected chemical and physical data for Rocky Mountain snowpack samples for 1993.—Continued

[E, depth-integrated environmental; VWM, volume-weighted mean of two snowpack layers; ANC, acid neutralization capacity; $\mu$ eq/L, microequivalents per liter; $\mu$ S/cm, microsiemens per centimeter; SC, specific conductance at $25^{\circ} \mathrm{C} ; \mathrm{pH}$ values in standard units; $\mathrm{H}$, hydrogen; $\mathrm{Ca}$, calcium; $\mathrm{Mg}$, magnesium; $\mathrm{Na}$, sodium; $\mathrm{K}$, potassium; $\mathrm{NH}_{4}$, ammonium; $\mathrm{Cl}_{\text {, chloride; }} \mathrm{SO}_{4}$, sulfate; $\mathrm{NO}_{3}$, nitrate; $\mathrm{DOC}$, dissolved organic carbon; mg/L, milligrams per liter; SWE, snow/water equivalent; cm, centimeters; $\delta^{34} \mathrm{~S}$, stable sulfur isotope ratio $\left.{ }^{34} \mathrm{~S} /{ }^{2} \mathrm{~S}\right) ; \mathrm{Hg}$, mercury; ng/L, nanograms per liter; na, not analyzed; <, below reporting limits; $\%$, percent]

\begin{tabular}{|c|c|c|c|c|c|c|c|c|c|c|c|c|c|c|c|c|c|c|c|c|}
\hline Site name & $\begin{array}{l}\text { Sample } \\
\text { date }\end{array}$ & $\begin{array}{c}\text { Sample } \\
\text { type }\end{array}$ & $\begin{array}{c}\text { ANC } \\
\text { ( } \mu \mathrm{eq} / \mathrm{L})\end{array}$ & $\begin{array}{c}\mathrm{SC} \\
(\mu \mathrm{S} / \mathrm{cm})\end{array}$ & $\mathrm{pH}$ & $\begin{array}{c}\mathrm{H} \\
(\mu \mathrm{eq} / \mathrm{L})\end{array}$ & $\begin{array}{c}\text { Ca } \\
(\mu \mathrm{eq} / \mathrm{L})\end{array}$ & $\underset{(\mu \mathrm{eq} / \mathrm{L})}{\mathrm{Mg}}$ & $\begin{array}{c}\mathrm{Na} \\
(\mu \mathrm{eq} / \mathrm{L})\end{array}$ & $\begin{array}{c}K \\
\text { ( } \mu \text { eq/L) }\end{array}$ & $\begin{array}{c}\mathrm{NH}_{4} \\
\text { ( } \mu \mathrm{eq} / \mathrm{L} \text { ) }\end{array}$ & $\begin{array}{c}C l \\
(\mu e q / L)\end{array}$ & $\begin{array}{c}\mathrm{SO}_{4} \\
(\mu \mathrm{eq} / \mathrm{L})\end{array}$ & 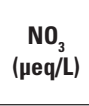 & $\begin{array}{l}\text { DOC } \\
\text { (mg/L) }\end{array}$ & $\begin{array}{l}\text { SWE } \\
(\mathrm{cm})\end{array}$ & $\begin{array}{l}\text { Snow } \\
\text { depth } \\
\text { (cm) }\end{array}$ & $\begin{array}{c}\delta^{34} \mathrm{~S} \\
\text { (per mil) }\end{array}$ & $\underset{(n g / L)}{\mathrm{Hg}}$ & $\begin{array}{c}\text { Ionic } \\
\text { balance } \\
(\%)\end{array}$ \\
\hline $\begin{array}{l}\text { Rabbit Ears 2, } \\
\text { Colo. }\end{array}$ & 4/6/1993 & VWM & na & 6.6 & 4.92 & 12.0 & $<3.1$ & $<1.7$ & $<1.0$ & $<0.4$ & 5.0 & $<1.0$ & 10.2 & 10.3 & $<0.4$ & 94 & 270 & 5.8 & na & -9.1 \\
\hline $\begin{array}{l}\text { Red Mountain, } \\
\text { Mont. }\end{array}$ & 4/7/1993 & E & -10.3 & 3.8 & 5.26 & 5.5 & $<3.1$ & $<1.7$ & $<1.0$ & $<0.4$ & 5.4 & $<1.0$ & 6.3 & 4.2 & 0.5 & 33 & 125 & 6.1 & na & 1.6 \\
\hline $\begin{array}{l}\text { Red Mountain } \\
\text { Pass, Colo. }\end{array}$ & $3 / 31 / 1993$ & E & -5.6 & 4.2 & 5.19 & 6.5 & 5.6 & $<1.7$ & 1.3 & $<0.4$ & 2.9 & $<1.0$ & 6.0 & 6.5 & $<0.4$ & 76 & 228 & 3.5 & na & 12.9 \\
\hline $\begin{array}{l}\text { Rendezvous } \\
\text { Mountain, } \\
\text { Wyo. }\end{array}$ & 4/6/1993 & E & 11.3 & 3.0 & 5.76 & 1.7 & 4.8 & 2.0 & 1.9 & $<0.4$ & 2.9 & $<1.0$ & 5.4 & 3.6 & $<0.4$ & 69 & 255 & 6.4 & na & -21.2 \\
\hline $\begin{array}{l}\text { Slumgullion } \\
\text { Pass, Colo. }\end{array}$ & $3 / 30 / 1993$ & E & 0.9 & 3.2 & 5.41 & 3.9 & 5.0 & $<1.7$ & 2.1 & na & 1.8 & $<1.0$ & 5.2 & 3.8 & 1.5 & 18 & 94 & 5.8 & na & 12.8 \\
\hline $\begin{array}{l}\text { Snow Bowl, } \\
\text { Mont. }\end{array}$ & 4/3/1993 & E & -9.3 & 3.3 & 5.35 & 4.5 & $<3.1$ & $<1.7$ & 1.5 & 1.1 & 3.6 & $<1.0$ & 5.0 & 3.4 & $<0.4$ & 48 & 145 & 4.5 & na & 11.5 \\
\hline $\begin{array}{l}\text { Snyder Lake, } \\
\text { Mont. }\end{array}$ & $3 / 31 / 1993$ & E & -2.2 & 4.4 & 5.18 & 6.6 & $<3.1$ & $<1.7$ & 1.9 & 2.9 & 3.2 & 1.7 & 5.6 & 4.7 & 1.3 & 42 & 131 & 7.4 & na & 10.0 \\
\hline $\begin{array}{l}\text { South Pass, } \\
\text { Wyo. }\end{array}$ & $3 / 24 / 1993$ & E & -16.4 & 6.2 & 4.94 & 11.5 & $<3.1$ & $<1.7$ & 1.7 & 0.6 & 2.9 & $<1.0$ & 9.4 & 7.1 & 0.4 & 33 & 116 & 7.2 & na & 0.3 \\
\hline $\begin{array}{l}\text { Sunlight Peak, } \\
\text { Colo. }\end{array}$ & $3 / 18 / 1993$ & E & -13.8 & 4.8 & 5.13 & 7.4 & 4.3 & 2.1 & 2.2 & 0.6 & 3.2 & $<1.0$ & 7.1 & 6.8 & $<0.4$ & 48 & 158 & 5.0 & na & 17.6 \\
\hline $\begin{array}{l}\text { Sylvan Lake, } \\
\text { Wyo. }\end{array}$ & $3 / 28 / 1993$ & E & -10.2 & 4.3 & 5.15 & 7.1 & $<3.1$ & $<1.7$ & 1.5 & $<0.4$ & 3.9 & $<1.0$ & 7.3 & 4.9 & $<0.4$ & 46 & 159 & 3.2 & na & 1.5 \\
\hline $\begin{array}{c}\text { Teton Pass, } \\
\text { Wyo. }\end{array}$ & $3 / 27 / 1993$ & E & 1.7 & 4.5 & 5.67 & 2.1 & 7.5 & 2.1 & 2.7 & 0.7 & 5.7 & 3.1 & 6.9 & 9.3 & 0.4 & 48 & 127 & 4.9 & na & -0.2 \\
\hline $\begin{array}{l}\text { Togwotee Pass, } \\
\text { Wyo. }\end{array}$ & $3 / 26 / 1993$ & E & -9.7 & 3.8 & 5.30 & 5.0 & $<3.1$ & $<1.7$ & 1.2 & 0.4 & 2.9 & $<1.0$ & 4.4 & 3.9 & $<0.4$ & 53 & 170 & 6.3 & na & 6.4 \\
\hline $\begin{array}{l}\text { Twenty-one } \\
\text { Mile, Mont. }\end{array}$ & 3/29/1993 & E & -15.7 & 6.3 & 4.91 & 12.3 & $<3.1$ & $<1.7$ & 1.7 & 0.7 & 5.0 & 1.7 & 6.9 & 7.3 & 0.4 & 38 & 102 & 4.3 & na & 10.8 \\
\hline $\begin{array}{l}\text { University } \\
\text { Camp, Colo. }\end{array}$ & $4 / 29 / 1993$ & E & na & 6.9 & 4.87 & 13.5 & 4.4 & 1.9 & 2.0 & 0.7 & 5.4 & $<1.0$ & 10.0 & 8.1 & 0.6 & 48 & 145 & 5.2 & na & 21.0 \\
\hline $\begin{array}{l}\text { West Yellow- } \\
\text { stone, Mont. }\end{array}$ & $3 / 29 / 1993$ & E & -13.5 & 4.3 & 5.18 & 6.6 & $<3.1$ & $<1.7$ & 1.3 & $<0.4$ & 5.0 & 1.4 & 4.4 & 6.9 & $<0.4$ & 31 & 85 & 4.6 & na & 0.7 \\
\hline $\begin{array}{l}\text { Wolf Creek } \\
\text { Pass, Colo. }\end{array}$ & $3 / 31 / 1993$ & E & -17.9 & 6.1 & 4.94 & 11.5 & $<3.1$ & 2.0 & 1.7 & $<0.4$ & 2.9 & $<1.0$ & 9.6 & 6.3 & $<0.4$ & 97 & 295 & 3.6 & na & 6.4 \\
\hline
\end{tabular}


Table 3. Selected chemical and physical data for Rocky Mountain snowpack samples for 1994.

[E, depth-integrated environmental; VWM, volume-weighted mean of two snowpack layers; ANC, acid neutralization capacity; ueq/L, microequivalents per liter; $u$ S/cm, microsiemens per centimeter; SC, specific conductance at $25^{\circ} \mathrm{C} ; \mathrm{pH}$ values in standard units; $\mathrm{H}$, hydrogen; $\mathrm{Ca}$, calcium; $\mathrm{Mg}$, magnesium; $\mathrm{Na}$, sodium; $\mathrm{K}$, potassium; $\mathrm{NH}_{4}$, ammonium; $\mathrm{Cl}_{\text {, chloride; }} \mathrm{SO}_{4}$, sulfate; $\mathrm{NO}_{3}$, nitrate; $\mathrm{DOC}_{\text {, dissolved organic }}$ carbon; $\mathrm{mg} / \mathrm{L}$, milligrams per liter; SWE, snow/water equivalent; $\mathrm{cm}$, centimeters; $\delta^{34} \mathrm{~S}$, stable sulfur isotope ratio $\left.{ }^{34} \mathrm{~S} /{ }^{2} \mathrm{~S}\right) ; \mathrm{Hg}$, mercury; ng/L, nanograms per liter; na, not analyzed; <, below reporting limits; $\%$, percent]

\begin{tabular}{|c|c|c|c|c|c|c|c|c|c|c|c|c|c|c|c|c|c|c|c|c|}
\hline Site name & $\begin{array}{l}\text { Sample } \\
\text { date }\end{array}$ & $\begin{array}{l}\text { Sample } \\
\text { type }\end{array}$ & $\begin{array}{c}\text { ANC } \\
(\mu \mathrm{eq} / \mathrm{L})\end{array}$ & $\begin{array}{c}S C \\
(\mu \mathrm{S} / \mathrm{cm})\end{array}$ & $\mathrm{pH}$ & $\begin{array}{c}H \\
(\mu e q / L)\end{array}$ & $\begin{array}{c}\mathrm{Ca} \\
(\mu \mathrm{eq} / \mathrm{L})\end{array}$ & $\begin{array}{c}M g \\
\text { (peq/L) }\end{array}$ & $\begin{array}{c}\mathrm{Na} \\
\text { (peq/L) }\end{array}$ & $\begin{array}{c}K \\
(\mu \mathrm{eq} / \mathrm{L})\end{array}$ & $\begin{array}{c}\mathrm{NH}_{4} \\
(\mu \mathrm{eq} / \mathrm{L})\end{array}$ & $\begin{array}{c}C l \\
(\mu e q / L)\end{array}$ & $\begin{array}{c}\mathrm{SO}_{4} \\
(\mu \mathrm{eq} / \mathrm{L})\end{array}$ & $\begin{array}{c}\mathrm{NO}_{3} \\
(\mu \mathrm{eq} / \mathrm{L})\end{array}$ & $\begin{array}{c}\text { DOC } \\
\text { (mg/L) }\end{array}$ & $\begin{array}{l}\text { SWE } \\
(\mathbf{c m})\end{array}$ & $\begin{array}{l}\text { Snow } \\
\text { depth } \\
(\mathrm{cm})\end{array}$ & $\begin{array}{l}\delta^{34} S \\
\text { (per } \\
\text { mil) }\end{array}$ & $\begin{array}{c}\mathrm{Hg} \\
\text { (ng/L) }\end{array}$ & $\begin{array}{c}\text { lonic } \\
\text { balance } \\
(\%)\end{array}$ \\
\hline $\begin{array}{l}\text { Berthoud Pass, } \\
\text { Colo. }\end{array}$ & 4/7/1994 & E & -12.8 & 5.5 & 4.94 & 11.5 & 4.5 & $<1.7$ & 2.2 & $<0.4$ & 2.5 & 1.7 & 4.6 & 9.1 & $<0.4$ & na & 185 & 5.9 & na & 14.5 \\
\hline $\begin{array}{l}\text { Big Mountain, } \\
\text { Mont. }\end{array}$ & $3 / 22 / 1994$ & E & -10.6 & 4.5 & 5.05 & 8.9 & $<3.1$ & $<1.7$ & 1.7 & $<0.4$ & 3.9 & $<1.0$ & 4.8 & 6.2 & 0.5 & na & 229 & 5.0 & na & 14.0 \\
\hline Big Sky, Mont. & $3 / 25 / 1994$ & E & 0.5 & 4.0 & 5.79 & 1.6 & 8.1 & $<1.7$ & 4.9 & 0.8 & 6.1 & 1.7 & 7.3 & 6.9 & 0.7 & na & 174 & 7.3 & na & 13.8 \\
\hline $\begin{array}{l}\text { Biscuit Basin, } \\
\text { Wyo. }\end{array}$ & $3 / 21 / 1994$ & E & 3.9 & 5.0 & 5.90 & 1.3 & 7.2 & $<1.7$ & 6.7 & 0.6 & 10.4 & 2.8 & 8.1 & 8.0 & 0.9 & na & 89 & na & na & 6.8 \\
\hline $\begin{array}{l}\text { Brooklyn } \\
\text { Lake, Wyo. }\end{array}$ & 3/31/1994 & E & -6.6 & 5.6 & 5.21 & 6.2 & 7.0 & 2.0 & 1.7 & 1.8 & 5.4 & 1.7 & 11.0 & 14.1 & 1.3 & na & 217 & 6.7 & na & -5.6 \\
\hline Brumley, Colo. & $3 / 31 / 1994$ & E & -6.7 & 5.0 & 5.24 & 5.8 & 9.5 & $<1.7$ & 1.7 & 1.1 & 4.3 & 2.0 & 5.4 & 10.2 & 0.6 & na & 97 & 5.4 & na & 11.9 \\
\hline $\begin{array}{l}\text { Buffalo Pass, } \\
\text { Colo. }\end{array}$ & 4/8/1994 & VWM & -20.2 & 10.6 & 4.75 & 17.6 & 9.8 & 2.2 & 1.9 & $<0.4$ & 10.9 & 1.4 & 19.8 & 17.3 & 0.6 & 85 & 260 & 7.2 & na & 4.8 \\
\hline $\begin{array}{l}\text { Cameron Pass, } \\
\text { Colo. }\end{array}$ & 4/8/1994 & E & -11.8 & 5.8 & 5.00 & 10.0 & 6.1 & $<1.7$ & 1.6 & 0.9 & 4.6 & 1.1 & 7.9 & 9.3 & 0.5 & na & 214 & 6.1 & na & 12.0 \\
\hline Canyon, Wyo. & 3/19/1994 & E & 1.7 & 4.3 & 5.86 & 1.4 & 5.0 & $<1.7$ & 5.2 & 1.7 & 8.2 & 2.3 & 7.5 & 7.0 & 0.7 & na & 85 & 6.4 & na & 7.6 \\
\hline $\begin{array}{l}\text { Chief Joseph } \\
\text { Pass, Mont. }\end{array}$ & $3 / 24 / 1994$ & E & -3.6 & 3.5 & 5.39 & 4.1 & $<3.1$ & $<1.7$ & 1.7 & 1.6 & 4.3 & 1.4 & 3.8 & 4.8 & 1.2 & na & 120 & 6.0 & na & 8.1 \\
\hline $\begin{array}{c}\text { Daisy Pass, } \\
\text { Mont. }\end{array}$ & $3 / 20 / 1994$ & E & -8.9 & 3.6 & 5.13 & 7.4 & $<3.1$ & $<1.7$ & 2.2 & $<0.4$ & 5.0 & 1.1 & 4.0 & 5.3 & $<0.4$ & na & 162 & 5.3 & na & 16.8 \\
\hline $\begin{array}{l}\text { Deadman Pass, } \\
\text { Colo. }\end{array}$ & 4/29/1994 & E & 15.2 & 5.3 & 6.20 & 0.6 & 10.2 & 4.3 & 1.1 & 6.9 & 3.9 & 2.8 & 7.7 & 7.7 & 5.3 & na & 70 & na & na & -10.6 \\
\hline $\begin{array}{l}\text { Divide Peak, } \\
\text { Wyo. }\end{array}$ & $3 / 30 / 1994$ & E & -9.2 & 6.2 & 5.13 & 7.4 & 7.8 & 1.8 & 1.6 & 0.5 & 7.1 & 1.1 & 9.8 & 13.1 & 0.5 & na & 122 & 6.6 & na & 4.4 \\
\hline $\begin{array}{l}\text { Dry Canyon, } \\
\text { Mont. }\end{array}$ & $3 / 23 / 1994$ & E & -1.0 & 4.9 & 5.61 & 2.5 & 7.9 & 1.9 & 2.7 & 0.6 & 14.3 & 3.1 & 7.3 & 8.6 & 0.7 & na & 145 & na & na & 22.3 \\
\hline $\begin{array}{c}\text { Dry Lake, } \\
\text { Mont. }\end{array}$ & $3 / 19 / 1994$ & VWM & -21.1 & 10.3 & 4.74 & 18.4 & 7.4 & 1.9 & 2.1 & $<0.4$ & 6.0 & 2.0 & 14.3 & 18.0 & 0.4 & 33 & 104 & 7.5 & na & 2.0 \\
\hline $\begin{array}{l}\text { Dunckley Pass, } \\
\text { Colo. }\end{array}$ & $3 / 24 / 1994$ & VWM & -7.3 & 5.0 & 5.16 & 6.9 & 12.6 & 2.5 & 1.7 & 1.1 & 4.5 & 2.4 & 6.9 & 13.7 & 0.4 & 69 & 175 & 6.0 & na & 12.0 \\
\hline $\begin{array}{l}\text { Elk River, } \\
\text { Colo. }\end{array}$ & $3 / 19 / 1994$ & VWM & -8.8 & 6.7 & 5.12 & 8.6 & 9.9 & 1.9 & 1.5 & 0.7 & 6.1 & 1.4 & 10.6 & 14.0 & 0.7 & 40 & 121 & 6.8 & na & 4.9 \\
\hline $\begin{array}{l}\text { Elkhart Park, } \\
\text { Wyo. }\end{array}$ & $3 / 26 / 1994$ & E & -6.9 & 4.0 & 5.27 & 5.4 & 3.5 & $<1.7$ & 2.2 & $<0.4$ & 4.6 & 1.1 & 5.6 & 6.4 & 0.4 & na & 116 & 6.6 & na & 8.6 \\
\hline $\begin{array}{l}\text { Four Mile } \\
\quad \text { Meadow, } \\
\text { Wyo. }\end{array}$ & $3 / 28 / 1994$ & E & -3.0 & 4.0 & 5.44 & 3.6 & 5.5 & $<1.7$ & 5.2 & 1.4 & 5.7 & 2.5 & 6.7 & 7.0 & 0.5 & na & 76 & 8.0 & na & 13.9 \\
\hline
\end{tabular}


Table 3. Selected chemical and physical data for Rocky Mountain snowpack samples for 1994.—Continued

[E, depth-integrated environmental; VWM, volume-weighted mean of two snowpack layers; ANC, acid neutralization capacity; $\mu$ eq/L, microequivalents per liter; $\mu$ S/cm, microsiemens per centimeter; SC, specific conductance at $25^{\circ} \mathrm{C} ; \mathrm{pH}$ values in standard units; $\mathrm{H}$, hydrogen; $\mathrm{Ca}$, calcium; $\mathrm{Mg}$, magnesium; $\mathrm{Na}$, sodium; $\mathrm{K}$, potassium; $\mathrm{NH}_{4}$, ammonium; $\mathrm{Cl}_{\text {, chloride; }} \mathrm{SO}_{4}$, sulfate; $\mathrm{NO}_{3}$, nitrate; $\mathrm{DOC}$, dissolved organic carbon; $\mathrm{mg} / \mathrm{L}$, milligrams per liter; SWE, snow/water equivalent; $\mathrm{cm}$, centimeters; $\delta^{34} \mathrm{~S}$, stable sulfur isotope ratio $\left.{ }^{34} \mathrm{~S} /{ }^{2} \mathrm{~S}\right) ; \mathrm{Hg}$, mercury; ng/L, nanograms per liter; na, not analyzed; <, below reporting limits; $\%$, percent]

\begin{tabular}{|c|c|c|c|c|c|c|c|c|c|c|c|c|c|c|c|c|c|c|c|c|}
\hline Site name & $\begin{array}{l}\text { Sample } \\
\text { date }\end{array}$ & $\begin{array}{l}\text { Sample } \\
\text { type }\end{array}$ & $\begin{array}{c}\text { ANC } \\
\text { ( } \mu \text { eq/L) }\end{array}$ & $\begin{array}{c}\mathrm{SC} \\
(\mu \mathrm{S} / \mathrm{cm})\end{array}$ & $\mathrm{pH}$ & $\underset{\text { (peq/L) }}{H}$ & $\begin{array}{c}\text { Ca } \\
\text { (peq/L) }\end{array}$ & 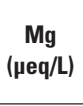 & $\begin{array}{c}\mathrm{Na} \\
(\mu \mathrm{eq} / \mathrm{L})\end{array}$ & 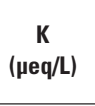 & 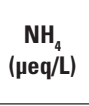 & $\begin{array}{c}C l \\
(\mu e q / L)\end{array}$ & $\begin{array}{c}\mathrm{SO}_{4} \\
(\mu \mathrm{eq} / \mathrm{L})\end{array}$ & 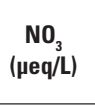 & $\begin{array}{c}\text { DOC } \\
\text { (mg/L) }\end{array}$ & $\begin{array}{l}\text { SWE } \\
(\mathrm{cm})\end{array}$ & $\begin{array}{l}\text { Snow } \\
\text { depth } \\
(\mathrm{cm})\end{array}$ & $\begin{array}{l}\delta^{34} S \\
\text { (per } \\
\text { mil) }\end{array}$ & $\underset{\text { (ng/L) }}{\mathrm{Hg}}$ & $\begin{array}{c}\text { lonic } \\
\text { balance } \\
(\%)\end{array}$ \\
\hline $\begin{array}{l}\text { Fremont Pass, } \\
\text { Colo. }\end{array}$ & 5/7/1994 & E & 15.6 & 5.1 & 6.25 & 0.6 & 16.7 & 5.0 & 2.6 & 7.7 & 4.3 & 3.7 & 6.3 & 9.9 & 8.7 & na & 105 & na & na & 2.0 \\
\hline $\begin{array}{l}\text { Gallegos Peak, } \\
\text { N. Mex. }\end{array}$ & $3 / 12 / 1994$ & E & -9.5 & 5.6 & 5.12 & 7.6 & 10.0 & $<1.7$ & 1.7 & 2.5 & 4.6 & 2.0 & 8.8 & 10.9 & 1.4 & na & 119 & 4.2 & na & 10.0 \\
\hline $\begin{array}{l}\text { Garnet Can- } \\
\text { yon, Wyo. }\end{array}$ & $3 / 26 / 1994$ & $\mathrm{E}$ & -6.6 & 3.6 & 5.21 & 6.2 & $<3.1$ & $<1.7$ & $<1.0$ & $<0.4$ & 4.3 & $<1.0$ & 4.2 & 4.6 & 0.5 & na & 190 & na & na & 8.5 \\
\hline $\begin{array}{l}\text { Grand Mesa, } \\
\text { Colo. }\end{array}$ & 4/20/1994 & E & -0.6 & 6.2 & 5.52 & 3.0 & 16.5 & 4.1 & 3.9 & 6.7 & 8.9 & 2.3 & 12.7 & 12.4 & 2.4 & na & 117 & 4.7 & na & 22.4 \\
\hline $\begin{array}{l}\text { Granite Pass, } \\
\text { Mont. }\end{array}$ & $3 / 23 / 1994$ & E & -5.8 & 3.3 & 5.27 & 5.4 & $<3.1$ & $<1.7$ & 1.7 & 0.5 & 2.1 & 1.4 & 3.1 & 3.0 & 0.7 & na & 148 & 5.4 & na & 12.8 \\
\hline $\begin{array}{l}\text { Gypsum } \\
\text { Creek, } \\
\text { Wyo. }\end{array}$ & 3/27/1994 & E & -0.4 & 4.5 & 5.70 & 2.0 & 7.6 & 1.8 & 3.9 & 0.8 & 6.8 & 2.0 & 6.9 & 8.8 & 0.6 & na & 78 & na & na & 12.9 \\
\hline $\begin{array}{l}\text { Hopewell, } \\
\text { N. Mex. }\end{array}$ & $3 / 13 / 1994$ & E & -8.4 & 5.0 & 5.14 & 7.2 & 8.0 & $<1.7$ & $<1.0$ & 1.3 & 4.3 & 2.0 & 9.4 & 8.7 & 0.8 & na & 130 & 3.6 & na & 1.8 \\
\hline $\begin{array}{c}\text { Kings Hill, } \\
\text { Mont. }\end{array}$ & $3 / 21 / 1994$ & E & -5.5 & 4.7 & 5.33 & 4.7 & 5.0 & $<1.7$ & $<1.0$ & 0.5 & 7.1 & 2.5 & 9.0 & 7.4 & 1.0 & na & 139 & 9.7 & na & -4.5 \\
\hline $\begin{array}{l}\text { Lake Irene, } \\
\text { Colo. }\end{array}$ & 3/30/1994 & E & -11.2 & 5.7 & 5.02 & 9.5 & 6.5 & $<1.7$ & 1.7 & $<0.4$ & 2.5 & 1.7 & 6.9 & 10.4 & 0.6 & na & 195 & 6.1 & na & 3.2 \\
\hline $\begin{array}{c}\text { Lewis Lake } \\
\text { Divide, } \\
\text { Wyo. }\end{array}$ & $3 / 17 / 1994$ & E & -2.5 & 3.8 & 5.59 & 2.6 & $<3.1$ & $<1.7$ & $<1.0$ & 0.4 & 10.0 & 1.1 & 5.0 & 7.4 & 0.4 & na & 160 & 5.2 & na & -2.2 \\
\hline $\begin{array}{c}\text { Lionshead, } \\
\text { Mont. }\end{array}$ & 3/18/1994 & E & -3.5 & 6.4 & 5.47 & 3.4 & 6.0 & $<1.7$ & 2.2 & 0.8 & 20.4 & 3.4 & 11.0 & 13.6 & 0.8 & na & 105 & 6.4 & na & 7.6 \\
\hline $\begin{array}{l}\text { Lionshead } \\
\text { North, } \\
\text { Mont. }\end{array}$ & 3/22/1994 & E & 12.3 & 7.3 & 6.13 & 0.7 & 13.4 & 3.0 & 2.6 & 13.6 & 15.4 & 8.5 & 9.0 & 10.1 & 2.0 & na & 114 & na & na & 10.0 \\
\hline $\begin{array}{l}\text { Loch Vale For- } \\
\text { est, Colo. }\end{array}$ & 4/6/1994 & E & -12.3 & 7.2 & 4.96 & 11.0 & 8.5 & 2.5 & 1.3 & 1.2 & 6.8 & 1.4 & 10.6 & 14.9 & 1.0 & 89 & 243 & 5.8 & na & 7.4 \\
\hline $\begin{array}{l}\text { Loveland Pass, } \\
\text { Colo. }\end{array}$ & $5 / 10 / 1994$ & E & -1.1 & 5.0 & 5.42 & 3.8 & 13.0 & 4.1 & 5.2 & 4.8 & 4.6 & 4.2 & 6.7 & 11.4 & 5.7 & na & 150 & 5.0 & na & 22.8 \\
\hline $\begin{array}{c}\text { Lynx Pass, } \\
\text { Colo. }\end{array}$ & $3 / 18 / 1994$ & E & -18.5 & 7.6 & 4.84 & 14.5 & 7.5 & $<1.7$ & 2.2 & $<0.4$ & 3.9 & 1.1 & 7.7 & 13.7 & 0.6 & na & 109 & 6.7 & na & 10.8 \\
\hline $\begin{array}{l}\text { Molas Lake, } \\
\text { Colo. }\end{array}$ & 4/1/1994 & $\mathrm{E}$ & 3.6 & 4.7 & 5.70 & 2.0 & 10.5 & $<1.7$ & 2.2 & 9.7 & 3.9 & 3.4 & 6.3 & 8.1 & 2.7 & na & 171 & 4.1 & na & 13.9 \\
\hline $\begin{array}{l}\text { Monarch Pass, } \\
\text { Colo. }\end{array}$ & 3/31/1994 & E & 5.6 & 5.9 & 5.85 & 1.4 & 20.0 & 3.3 & $<1.0$ & 4.9 & 5.7 & 2.8 & 9.0 & 11.7 & 2.7 & na & 106 & 4.8 & na & 9.6 \\
\hline
\end{tabular}


Table 3. Selected chemical and physical data for Rocky Mountain snowpack samples for 1994.—Continued

[E, depth-integrated environmental; VWM, volume-weighted mean of two snowpack layers; ANC, acid neutralization capacity; ueq/L, microequivalents per liter; $\mu \mathrm{S} / \mathrm{cm}$, microsiemens per centimeter; SC, specific conductance at $25^{\circ} \mathrm{C} ; \mathrm{pH}$ values in standard units; $\mathrm{H}$, hydrogen; $\mathrm{Ca}$, calcium; $\mathrm{Mg}$, magnesium; $\mathrm{Na}$, sodium; $\mathrm{K}$, potassium; $\mathrm{NH}_{4}$, ammonium; $\mathrm{Cl}_{\text {, chloride; }} \mathrm{SO}_{4}$, sulfate; $\mathrm{NO}$, nitrate; $\mathrm{DOC}$, dissolved organic carbon; mg/L, milligrams per liter; SWE, snow/water equivalent; cm, centimeters; $\delta^{34} \mathrm{~S}$, stable sulfur isotope ratio $\left.{ }^{34} \mathrm{~S} /{ }^{2} \mathrm{~S}\right) ; \mathrm{Hg}$, mercury; ng/L, nanograms per liter; na, not analyzed; <, below reporting limits; $\%$, percent]

\begin{tabular}{|c|c|c|c|c|c|c|c|c|c|c|c|c|c|c|c|c|c|c|c|c|}
\hline Site name & $\begin{array}{l}\text { Sample } \\
\text { date }\end{array}$ & $\begin{array}{c}\text { Sample } \\
\text { type }\end{array}$ & $\begin{array}{c}\text { ANC } \\
(\mu \mathrm{eq} / \mathrm{L})\end{array}$ & $\underset{(\mu \mathrm{SC} / \mathrm{cm})}{\mathrm{SC}}$ & $\mathrm{pH}$ & $\begin{array}{c}H \\
(\mu \mathrm{eq} / \mathrm{L})\end{array}$ & $\underset{\text { ( } \mu \mathrm{eq} / \mathrm{L})}{\mathrm{Ca}}$ & 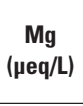 & $\begin{array}{c}\mathrm{Na} \\
\text { (peq/L) }\end{array}$ & $\begin{array}{c}K \\
\text { (peq/L) }\end{array}$ & 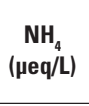 & $\underset{(\mu \mathrm{eq} / \mathrm{L})}{\mathrm{Cl}}$ & $\begin{array}{c}\mathrm{SO}_{4} \\
(\mu \mathrm{eq} / \mathrm{L})\end{array}$ & $\begin{array}{c}\mathrm{NO}_{3} \\
(\mu \mathrm{eq} / \mathrm{L})\end{array}$ & $\begin{array}{c}\text { DOC } \\
\text { (mg/L) }\end{array}$ & $\begin{array}{l}\text { SWE } \\
\text { (cm) }\end{array}$ & $\begin{array}{l}\text { Snow } \\
\text { depth } \\
\text { (cm) }\end{array}$ & $\begin{array}{l}\delta^{34} S \\
\text { (per } \\
\text { mil) }\end{array}$ & $\begin{array}{c}\mathrm{Hg} \\
\text { (ng/L) }\end{array}$ & $\begin{array}{c}\text { lonic } \\
\text { balance } \\
(\%)\end{array}$ \\
\hline $\begin{array}{l}\text { Moose } \\
\text { Meadow, } \\
\text { Idaho }\end{array}$ & $3 / 22 / 1994$ & E & 3.9 & 5.3 & 5.99 & 1.0 & 6.1 & $<1.7$ & 2.6 & 1.5 & 18.2 & 3.9 & 8.3 & 9.1 & 0.8 & na & 150 & na & na & 7.8 \\
\hline $\begin{array}{l}\text { Ned Wilson, } \\
\text { Colo. }\end{array}$ & $3 / 31 / 1994$ & VWM & -5.1 & 4.6 & 5.33 & 4.6 & 8.5 & $<1.7$ & 1.4 & 0.5 & 3.4 & 1.2 & 7.1 & 10.1 & 0.5 & 86 & 227 & 5.0 & na & -0.2 \\
\hline $\begin{array}{l}\text { Niwot Snotel, } \\
\text { Colo. }\end{array}$ & 4/4/1994 & E & -10.6 & 6.6 & 5.04 & 9.1 & 8.0 & $<1.7$ & $<1.0$ & 0.5 & 11.1 & 1.1 & 9.8 & 13.7 & 0.9 & na & 137 & 5.8 & na & 7.6 \\
\hline $\begin{array}{l}\text { Noisy Basin, } \\
\text { Mont. }\end{array}$ & $3 / 22 / 1994$ & E & -8.5 & 5.0 & 5.09 & 8.1 & $<3.1$ & $<1.7$ & $<1.0$ & 0.5 & 5.7 & 1.4 & 5.4 & 6.1 & 0.9 & na & 209 & 5.3 & na & 5.3 \\
\hline $\begin{array}{c}\text { Old Battle, } \\
\text { Wyo. }\end{array}$ & 3/29/1994 & E & -4.0 & 6.2 & 5.38 & 4.2 & 9.5 & 2.5 & 1.3 & 1.9 & 6.8 & 1.7 & 11.7 & 13.2 & 1.3 & na & 220 & 7.2 & na & -0.9 \\
\hline $\begin{array}{l}\text { Old Faithful } \\
\text { Crew Cor- } \\
\text { rals, Wyo. }\end{array}$ & $3 / 21 / 1994$ & E & 5.9 & 5.5 & 6.02 & 1.0 & 10.2 & 1.8 & 11.7 & 0.8 & 11.4 & 5.4 & 11.0 & 8.8 & 1.0 & na & 81 & na & na & 8.6 \\
\hline $\begin{array}{l}\text { Old Faithful } \\
\text { East Lot, } \\
\text { Wyo. }\end{array}$ & $3 / 21 / 1994$ & E & 2.8 & 5.1 & 5.84 & 1.4 & 8.8 & $<1.7$ & 12.3 & 0.5 & 9.3 & 5.1 & 10.2 & 7.5 & 0.5 & na & 92 & na & na & 11.6 \\
\hline $\begin{array}{l}\text { Old Faithful } \\
\text { Fire Road, } \\
\text { Wyo. }\end{array}$ & $3 / 21 / 1994$ & E & -0.9 & 4.7 & 5.59 & 2.6 & 7.9 & $<1.7$ & 7.9 & 0.6 & 7.5 & 3.7 & 9.0 & 7.4 & 0.8 & na & 91 & na & na & 13.7 \\
\hline $\begin{array}{l}\text { Phantom Val- } \\
\text { ley, Colo. }\end{array}$ & $3 / 25 / 1994$ & E & -15.8 & 7.3 & 4.86 & 13.8 & 7.9 & 2.0 & 2.0 & 0.8 & 5.7 & 1.1 & 9.6 & 13.1 & 1.0 & na & 96 & 6.2 & na & 15.1 \\
\hline $\begin{array}{l}\text { Rabbit Ears 1, } \\
\text { Colo. }\end{array}$ & 3/23/1994 & VWM & -13.3 & 8.0 & 4.89 & 12.8 & 9.7 & 2.0 & 2.6 & $<0.4$ & 6.2 & 2.2 & 12.3 & 14.2 & 0.5 & 88 & 237 & na & na & 7.4 \\
\hline $\begin{array}{l}\text { Rabbit Ears 2, } \\
\text { Colo. }\end{array}$ & $3 / 23 / 1994$ & vwM & -13.8 & 6.1 & 4.93 & 11.7 & 9.3 & 2.0 & 3.8 & $<0.4$ & 6.2 & 1.8 & 11.6 & 14.0 & 0.4 & 85 & 220 & 7.4 & na & 9.2 \\
\hline $\begin{array}{l}\text { Red Mountain, } \\
\text { Mont. }\end{array}$ & $3 / 24 / 1994$ & E & -0.6 & 4.0 & 5.57 & 2.7 & 8.9 & 2.3 & 2.9 & 1.9 & 6.1 & 1.4 & 6.0 & 7.1 & 1.3 & na & 109 & 8.2 & na & 25.8 \\
\hline $\begin{array}{l}\text { Red Mountain } \\
\text { Pass, Colo. }\end{array}$ & 4/1/1994 & E & -4.0 & 4.5 & 5.38 & 4.2 & 10.5 & $<1.7$ & $<1.0$ & 1.6 & 3.6 & 1.4 & 6.0 & 8.6 & 1.1 & na & 157 & 4.4 & na & 10.4 \\
\hline $\begin{array}{l}\text { Rendezvous } \\
\text { Mountain, } \\
\text { Wyo. }\end{array}$ & $3 / 25 / 1994$ & E & -6.5 & 3.3 & 5.21 & 6.2 & $<3.1$ & $<1.7$ & $<1.0$ & 0.7 & 3.6 & $<1.0$ & 4.0 & 4.2 & $<0.4$ & na & 152 & 5.2 & na & 12.2 \\
\hline $\begin{array}{l}\text { Slumgullion } \\
\text { Pass, Colo. }\end{array}$ & 4/2/1994 & E & -0.4 & 4.0 & 5.59 & 2.6 & 9.9 & 2.0 & 1.5 & 3.7 & 2.9 & 2.5 & 5.8 & 7.2 & 1.8 & na & 86 & na & na & 18.3 \\
\hline $\begin{array}{c}\text { Snow Bowl, } \\
\text { Mont. }\end{array}$ & $3 / 23 / 1994$ & E & -10.2 & 5.4 & 5.07 & 8.5 & $<3.1$ & $<1.7$ & 1.3 & 1.5 & 5.0 & 2.5 & 6.3 & 6.2 & 0.9 & na & 158 & 5.4 & na & 4.2 \\
\hline
\end{tabular}


Table 3. Selected chemical and physical data for Rocky Mountain snowpack samples for 1994.—Continued

[E, depth-integrated environmental; VWM, volume-weighted mean of two snowpack layers; ANC, acid neutralization capacity; $\mu$ eq/L, microequivalents per liter; $\mu$ S/cm, microsiemens per centimeter; SC, specific conductance at $25^{\circ} \mathrm{C} ; \mathrm{pH}$ values in standard units; $\mathrm{H}$, hydrogen; $\mathrm{Ca}$, calcium; $\mathrm{Mg}$, magnesium; $\mathrm{Na}$, sodium; $\mathrm{K}$, potassium; $\mathrm{NH}_{4}$, ammonium; $\mathrm{Cl}_{\text {, chloride; }} \mathrm{SO}_{4}$, sulfate; $\mathrm{NO}_{3}$, nitrate; $\mathrm{DOC}$, dissolved organic carbon; mg/L, milligrams per liter; SWE, snow/water equivalent; cm, centimeters; $\delta^{34} \mathrm{~S}$, stable sulfur isotope ratio $\left.{ }^{34} \mathrm{~S} /{ }^{2} \mathrm{~S}\right) ; \mathrm{Hg}$, mercury; ng/L, nanograms per liter; na, not analyzed; <, below reporting limits; $\%$, percent]

\begin{tabular}{|c|c|c|c|c|c|c|c|c|c|c|c|c|c|c|c|c|c|c|c|c|}
\hline Site name & $\begin{array}{l}\text { Sample } \\
\text { date }\end{array}$ & $\begin{array}{c}\text { Sample } \\
\text { type }\end{array}$ & 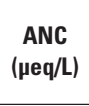 & $\begin{array}{c}\text { SC } \\
(\mu \mathrm{S} / \mathrm{cm})\end{array}$ & $\mathrm{pH}$ & 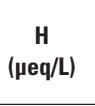 & $\begin{array}{c}\text { Ca } \\
\text { (peq/L) }\end{array}$ & $\underset{(\mu \mathrm{eq} / \mathrm{L})}{\mathrm{Mg}}$ & 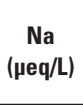 & 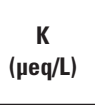 & 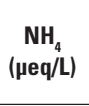 & $\underset{(\mu e q / L)}{C l}$ & $\begin{array}{c}\mathrm{SO}_{4} \\
(\mu \mathrm{eq} / \mathrm{L})\end{array}$ & $\begin{array}{c}\mathrm{NO}_{3} \\
(\mu \mathrm{eq} / \mathrm{L})\end{array}$ & $\begin{array}{c}\text { DOC } \\
(\mathrm{mg} / \mathrm{L})\end{array}$ & $\begin{array}{l}\text { SWE } \\
\text { (cm) }\end{array}$ & $\begin{array}{l}\text { Snow } \\
\text { depth } \\
(\mathrm{cm})\end{array}$ & $\begin{array}{l}\delta^{34} S \\
\text { (per } \\
\text { mil) }\end{array}$ & $\underset{(\mathrm{ng} / \mathrm{L})}{\mathrm{Hg}}$ & $\begin{array}{c}\text { lonic } \\
\text { balance } \\
(\%)\end{array}$ \\
\hline $\begin{array}{l}\text { South Pass, } \\
\text { Wyo. }\end{array}$ & $3 / 28 / 1994$ & E & -6.2 & 5.4 & 5.22 & 6.0 & 7.5 & $<1.7$ & 3.0 & 0.4 & 5.0 & 1.1 & 10.0 & 9.4 & 0.8 & na & 134 & 7.5 & na & 3.3 \\
\hline $\begin{array}{l}\text { Sunlight Peak, } \\
\text { Colo. }\end{array}$ & 4/20/1994 & E & -7.0 & 6.1 & 5.16 & 6.9 & 10.5 & 2.5 & $<1.0$ & 2.8 & 7.1 & 1.4 & 8.5 & 11.5 & 1.6 & na & 127 & 5.2 & na & 16.3 \\
\hline $\begin{array}{l}\text { Sylvan Lake, } \\
\text { Wyo. }\end{array}$ & $3 / 19 / 1994$ & E & -8.5 & 4.3 & 5.12 & 7.6 & $<3.1$ & $<1.7$ & 1.3 & 0.7 & 7.9 & 2.0 & 5.0 & 6.1 & 0.6 & na & 115 & 5.3 & na & 14.2 \\
\hline Targee, Mont. & $3 / 22 / 1994$ & E & -1.1 & 4.5 & 5.65 & 2.2 & 5.3 & $<1.7$ & 2.2 & 1.4 & 13.6 & 2.3 & 7.5 & 9.3 & 0.8 & na & 102 & na & na & 12.9 \\
\hline $\begin{array}{l}\text { Teton Pass, } \\
\text { Wyo. }\end{array}$ & 3/26/1994 & E & 33.7 & 9.5 & 6.61 & 0.2 & 24.0 & 9.1 & 10.0 & 5.7 & 13.2 & 7.0 & 11.7 & 12.4 & 2.6 & na & 156 & 7.2 & na & -2.1 \\
\hline $\begin{array}{l}\text { Togwotee Pass, } \\
\text { Wyo. }\end{array}$ & $3 / 28 / 1994$ & E & 1.7 & 4.1 & 5.88 & 1.3 & 7.5 & $<1.7$ & 5.7 & 0.5 & 5.7 & 2.3 & 7.3 & 7.8 & 0.6 & na & 156 & 7.1 & na & 4.1 \\
\hline $\begin{array}{l}\text { Trappers Peak, } \\
\text { Colo. }\end{array}$ & 3/30/1994 & VWM & -7.5 & 4.3 & 5.20 & 6.1 & 7.8 & $<1.7$ & 1.6 & $<0.4$ & 3.3 & 1.7 & 5.9 & 9.7 & $<0.4$ & 82 & 216 & 5.3 & na & 4.4 \\
\hline $\begin{array}{l}\text { Twenty-one } \\
\text { Mile, Mont. }\end{array}$ & 3/18/1994 & E & -6.0 & 4.3 & 5.23 & 5.9 & $<3.1$ & $<1.7$ & $<1.0$ & 0.5 & 8.2 & 3.7 & 5.6 & 7.1 & 0.6 & na & 90 & 6.0 & na & -5.7 \\
\hline $\begin{array}{l}\text { Two-Top } \\
\text { South, } \\
\text { Mont. }\end{array}$ & $3 / 22 / 1994$ & E & -0.3 & 5.0 & 5.73 & 1.9 & 5.2 & $<1.7$ & 2.2 & $<0.4$ & 20.7 & 2.3 & 9.6 & 11.1 & 0.5 & na & 166 & na & na & 13.3 \\
\hline $\begin{array}{l}\text { University } \\
\text { Camp, } \\
\text { Colo. }\end{array}$ & 4/27/1994 & E & -10.2 & 6.1 & 5.09 & 8.1 & 8.0 & $<1.7$ & $<1.0$ & 1.9 & 6.8 & 1.4 & 9.8 & 10.9 & 1.5 & na & 170 & 5.5 & na & 5.8 \\
\hline $\begin{array}{l}\text { West Yellow- } \\
\text { stone, } \\
\text { Mont. }\end{array}$ & $3 / 17 / 1994$ & E & 3.2 & 4.9 & 5.86 & 1.4 & 4.0 & $<1.7$ & 1.3 & 0.5 & 12.9 & 3.4 & 7.5 & 10.4 & 0.7 & na & 66 & 6.4 & na & -9.9 \\
\hline $\begin{array}{l}\text { West Yellow- } \\
\text { stone East, } \\
\text { Mont. }\end{array}$ & 3/25/1994 & E & -2.3 & 4.7 & 5.53 & 3.0 & 12.2 & 1.9 & 4.7 & 0.6 & 12.9 & 2.3 & 6.5 & 10.7 & 0.7 & na & 82 & na & na & 28.9 \\
\hline $\begin{array}{l}\text { Wolf Creek } \\
\text { Pass, Colo. }\end{array}$ & 4/2/1994 & E & -9.5 & 6.4 & 5.08 & 8.3 & 10.5 & $<1.7$ & $<1.0$ & 2.1 & 5.4 & 3.4 & $<0.6$ & 11.2 & 1.1 & na & 170 & 4.0 & na & 28.5 \\
\hline
\end{tabular}


[E, depth-integrated environmental; VWM, volume-weighted mean of two snowpack layers; ANC, acid neutralization capacity; $\mu$ eq/L, microequivalents per liter; $\mu \mathrm{S} / \mathrm{cm}$, microsiemens per centimeter; SC, specific conductance at $25^{\circ} \mathrm{C} ; \mathrm{pH}$ values in standard units; $\mathrm{H}$, hydrogen; $\mathrm{Ca}$, calcium; $\mathrm{Mg}$, magnesium; $\mathrm{Na}$, sodium; $\mathrm{K}$, potassium; $\mathrm{NH}_{4}$, ammonium; $\mathrm{Cl}$, chloride; $\mathrm{SO}_{4}$, sulfate; $\mathrm{NO}{ }_{3}$, nitrate; $\mathrm{DOC}$, dissolved organic carbon; $\mathrm{mg} / \mathrm{L}$, milligrams per liter; SWE, snow/water equivalent; cm, centimeters; $\delta^{34} \mathrm{~S}$, stable sulfur isotope ratio $\left.{ }^{34} \mathrm{~S} /{ }^{32} \mathrm{~S}\right) ; \mathrm{Hg}$, mercury; ng/L, nanograms per liter; na, not analyzed; <, below reporting limits; \%, percent]

\begin{tabular}{|c|c|c|c|c|c|c|c|c|c|c|c|c|c|c|c|c|c|c|c|c|}
\hline Site name & $\begin{array}{l}\text { Sample } \\
\text { date }\end{array}$ & $\begin{array}{c}\text { Sample } \\
\text { type }\end{array}$ & 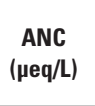 & $\begin{array}{c}\mathrm{SC} \\
(\mu \mathrm{S} / \mathrm{cm})\end{array}$ & $\mathrm{pH}$ & 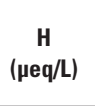 & $\begin{array}{c}\text { Ca } \\
(\mu \mathrm{eq} / \mathrm{L})\end{array}$ & $\underset{\text { (peq/L) }}{M g}$ & $\begin{array}{c}\mathrm{Na} \\
(\mu \mathrm{eq} / \mathrm{L})\end{array}$ & 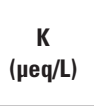 & $\begin{array}{c}\mathrm{NH}_{4} \\
(\mu \mathrm{eq} / \mathrm{L})\end{array}$ & 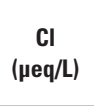 & 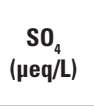 & $\begin{array}{c}\mathrm{NO}_{3} \\
(\mu \mathrm{eq} / \mathrm{L})\end{array}$ & $\begin{array}{c}\text { DOC } \\
\text { (mg/L) }\end{array}$ & $\begin{array}{l}\text { SWE } \\
(\mathrm{cm})\end{array}$ & $\begin{array}{l}\text { Snow } \\
\text { depth } \\
(\mathrm{cm})\end{array}$ & $\begin{array}{c}\delta^{34} \mathrm{~S} \\
\text { (per mil) }\end{array}$ & $\begin{array}{c}\mathrm{Hg} \\
\text { (ng/L) }\end{array}$ & $\begin{array}{c}\text { lonic } \\
\text { balance } \\
(\%)\end{array}$ \\
\hline $\begin{array}{l}\text { Berthoud Pass, } \\
\text { Colo. }\end{array}$ & 4/5/1995 & E & -6.5 & 3.7 & 5.18 & 6.6 & $<3.1$ & $<1.7$ & 4.1 & $<0.4$ & 2.9 & 1.7 & 4.4 & 7.0 & 0.4 & na & 144 & 6.0 & na & 2.0 \\
\hline $\begin{array}{l}\text { Big Mountain, } \\
\text { Mont. }\end{array}$ & 3/16/1995 & E & -4.5 & 3.0 & 5.19 & 6.5 & $<3.1$ & $<1.7$ & $<1.0$ & 0.5 & 3.3 & $<1.0$ & 3.8 & 4.4 & 0.7 & na & 266 & 5.2 & na & 11.7 \\
\hline Big Sky, Mont. & 3/19/1995 & E & -0.7 & 2.6 & 5.42 & 3.8 & 4.5 & $<1.7$ & $<1.0$ & 0.5 & 4.4 & $<1.0$ & 4.2 & 5.1 & 0.7 & na & 196 & 5.0 & na & 17.5 \\
\hline $\begin{array}{l}\text { Biscuit Basin, } \\
\text { Wyo. }\end{array}$ & $3 / 14 / 1995$ & E & -6.5 & 3.9 & 5.16 & 6.9 & $<3.1$ & $<1.7$ & $<1.0$ & $<0.4$ & 6.1 & 1.1 & 4.6 & 8.0 & 1.1 & na & 99 & na & na & -2.7 \\
\hline $\begin{array}{l}\text { Brooklyn Lake, } \\
\text { Wyo. }\end{array}$ & 3/25/1995 & E & -8.3 & 5.5 & 5.00 & 10.0 & 6.5 & $<1.7$ & $<1.0$ & 0.8 & 5.2 & 1.1 & 8.8 & 9.7 & 1.2 & na & 173 & 6.0 & na & 6.8 \\
\hline Brumley, Colo. & $3 / 31 / 1995$ & E & -6.6 & na & 5.18 & 6.6 & 3.5 & $<1.7$ & $<1.0$ & $<0.4$ & 2.5 & $<1.0$ & 2.9 & 6.1 & 0.8 & na & 134 & 4.9 & na & 16.3 \\
\hline $\begin{array}{l}\text { Buffalo Pass, } \\
\text { Colo. }\end{array}$ & 4/5/1995 & VWM & -13.7 & 6.9 & 4.90 & 13.9 & 5.0 & $<1.7$ & 1.0 & $<0.4$ & 5.8 & 1.3 & 12.1 & 10.8 & 0.6 & 112 & 280 & 7.5 & na & 2.9 \\
\hline $\begin{array}{l}\text { Buffalo Pass, } \\
\text { Colo. }\end{array}$ & $6 / 1 / 1995$ & E & -12.3 & 8.0 & 4.89 & 12.9 & 8.5 & $<1.7$ & $<1.0$ & 0.5 & 7.6 & 1.1 & 15.2 & 12.3 & 0.9 & 166 & 385 & 6.6 & na & 1.4 \\
\hline $\begin{array}{l}\text { Cameron Pass, } \\
\text { Colo. }\end{array}$ & 4/6/1995 & E & -10.3 & 5.8 & 5.00 & 10.0 & 6.9 & $<1.7$ & 3.8 & 1.3 & 4.6 & 3.1 & 8.3 & 12.8 & 1.4 & na & 171 & 6.5 & na & 4.7 \\
\hline Canyon, Wyo. & $3 / 12 / 1995$ & E & -5.9 & 3.3 & 5.14 & 7.2 & $<3.1$ & $<1.7$ & $<1.0$ & $<0.4$ & 4.4 & $<1.0$ & 3.3 & 5.6 & 0.4 & na & 128 & 4.1 & na & 12.8 \\
\hline $\begin{array}{l}\text { Chief Joseph } \\
\text { Pass, Mont. }\end{array}$ & 3/18/1995 & E & -4.0 & 2.5 & 5.32 & 4.8 & $<3.1$ & $<1.7$ & $<1.0$ & $<0.4$ & 3.8 & $<1.0$ & 2.9 & 3.3 & 0.6 & na & 150 & 4.1 & na & 16.0 \\
\hline $\begin{array}{c}\text { Daisy Pass, } \\
\text { Mont. }\end{array}$ & $3 / 14 / 1995$ & E & -3.5 & 2.9 & 5.29 & 5.1 & $<3.1$ & $<1.7$ & $<1.0$ & $<0.4$ & 3.7 & $<1.0$ & 4.0 & 4.3 & 0.5 & na & 250 & 4.2 & na & 3.4 \\
\hline $\begin{array}{l}\text { Deadman Pass, } \\
\text { Colo. }\end{array}$ & 4/14/1995 & E & -8.4 & 6.5 & 4.99 & 10.2 & 12.5 & 2.5 & $<1.0$ & 2.8 & 6.6 & 1.4 & 10.8 & 15.6 & 2.3 & na & 142 & 6.0 & na & 10.8 \\
\hline $\begin{array}{c}\text { Divide Peak, } \\
\text { Wyo. }\end{array}$ & 3/24/1995 & E & -8.6 & 6.2 & 5.05 & 8.9 & 13.0 & 2.5 & 1.7 & 0.5 & 6.1 & 2.0 & 10.2 & 15.9 & 0.5 & na & 139 & 6.6 & na & 7.6 \\
\hline $\begin{array}{c}\text { Dry Lake, } \\
\text { Colo. }\end{array}$ & 3/13/1995 & VWM & -21.0 & 9.4 & 4.69 & 20.6 & 6.1 & $<1.7$ & 1.6 & $<0.4$ & 6.8 & 1.2 & 12.9 & 18.9 & 0.6 & 42 & 122 & 7.2 & na & 3.2 \\
\hline $\begin{array}{l}\text { Dunckley Pass, } \\
\text { Colo. }\end{array}$ & 3/30/1995 & VWM & -0.1 & 4.2 & 5.43 & 3.8 & 15.8 & 2.9 & 1.2 & 4.5 & 4.4 & 1.2 & 8.0 & 11.7 & 2.1 & 44 & 152 & 5.3 & na & 22.0 \\
\hline Elk River, Colo. & 3/13/1995 & VWM & -12.3 & 6.8 & 4.98 & 11.0 & 8.1 & $<1.7$ & $<1.0$ & $<0.4$ & 5.5 & 1.4 & 9.8 & 13.9 & 0.7 & 45 & 130 & 7.0 & na & -0.9 \\
\hline $\begin{array}{c}\text { Elkhart Park, } \\
\text { Wyo. }\end{array}$ & 3/23/1995 & E & -7.4 & 5.0 & 5.08 & 8.3 & 4.0 & $<1.7$ & 1.7 & $<0.4$ & 4.2 & $<1.0$ & 8.5 & 7.4 & 0.7 & na & 142 & 7.3 & na & 6.7 \\
\hline $\begin{array}{l}\text { Four Mile } \\
\text { Meadow, } \\
\text { Wyo. }\end{array}$ & $3 / 21 / 1995$ & E & -4.9 & 3.5 & 5.20 & 6.3 & $<3.1$ & $<1.7$ & $<1.0$ & 0.5 & 3.6 & $<1.0$ & 3.3 & 7.0 & 0.8 & na & 120 & 5.7 & na & 0.3 \\
\hline
\end{tabular}


Table 4. Selected chemical and physical data for Rocky Mountain snowpack samples for 1995.—Continued

[E, depth-integrated environmental; VWM, volume-weighted mean of two snowpack layers; ANC, acid neutralization capacity; $\mu$ eq/L, microequivalents per liter; $\mu \mathrm{S} / \mathrm{cm}$, microsiemens per centimeter; SC, specific conductance at $25^{\circ} \mathrm{C} ; \mathrm{pH}$ values in standard units; $\mathrm{H}$, hydrogen; $\mathrm{Ca}$, calcium; $\mathrm{Mg}$, magnesium; $\mathrm{Na}$, sodium; $\mathrm{K}$, potassium; $\mathrm{NH}$, ammonium; $\mathrm{Cl}$, chloride; $\mathrm{SO}$, sulfate; $\mathrm{NO}{ }_{3}$, nitrate; $\mathrm{DOC}$, dissolved organic carbon; $\mathrm{mg} / \mathrm{L}$, milligrams per liter; SWE, snow/water equivalent; cm, centimeters; $\delta^{34} \mathrm{~S}$, stable sulfur isotope ratio $\left.{ }^{(34} \mathrm{S} /{ }^{32} \mathrm{~S}\right) ; \mathrm{Hg}$, mercury; ng/L, nanograms per liter; na, not analyzed; <, below reporting limits; \%, percent]

\begin{tabular}{|c|c|c|c|c|c|c|c|c|c|c|c|c|c|c|c|c|c|c|c|c|}
\hline Site name & $\begin{array}{l}\text { Sample } \\
\text { date }\end{array}$ & $\begin{array}{c}\text { Sample } \\
\text { type }\end{array}$ & $\begin{array}{c}\text { ANC } \\
(\mu \mathrm{eq} / \mathrm{L})\end{array}$ & $\begin{array}{c}\mathrm{SC} \\
(\mu \mathrm{S} / \mathrm{cm})\end{array}$ & $\mathrm{pH}$ & 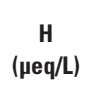 & $\underset{\text { (peq/L) }}{C a}$ & $\underset{\text { (peq/L) }}{M g}$ & $\underset{\text { (peq/L) }}{\mathrm{Na}}$ & $\begin{array}{c}K \\
(\mu e q / L)\end{array}$ & $\begin{array}{c}\mathrm{NH}_{4} \\
\text { ( } \mu \mathrm{eq} / \mathrm{L} \text { ) }\end{array}$ & $\underset{(\mu e q / L)}{C l}$ & $\begin{array}{c}\mathrm{SO}_{4} \\
(\mu \mathrm{eq} / \mathrm{L})\end{array}$ & $\begin{array}{c}\mathrm{NO}_{3} \\
(\mu \mathrm{eq} / \mathrm{L})\end{array}$ & $\begin{array}{l}\text { DOC } \\
\text { (mg/L) }\end{array}$ & $\begin{array}{l}\text { SWE } \\
\text { (cm) }\end{array}$ & $\begin{array}{l}\text { Snow } \\
\text { depth } \\
\text { (cm) }\end{array}$ & $\begin{array}{c}\delta^{34} \mathbf{S} \\
\text { (per mil) }\end{array}$ & $\underset{(\mathrm{ng} / \mathrm{L})}{\mathrm{Hg}}$ & $\begin{array}{c}\text { lonic } \\
\text { balance } \\
(\%)\end{array}$ \\
\hline $\begin{array}{l}\text { Fremont Pass, } \\
\text { Colo. }\end{array}$ & $4 / 24 / 1995$ & E & -4.4 & 3.5 & 5.21 & 6.2 & 5.5 & $<1.7$ & $<1.0$ & 0.5 & 2.7 & $<1.0$ & 3.8 & 7.4 & 0.7 & na & 175 & 3.9 & na & 14.5 \\
\hline $\begin{array}{l}\text { Gallegos Peak, } \\
\text { N. Mex. }\end{array}$ & $3 / 25 / 1995$ & E & -4.2 & 4.6 & 5.18 & 6.6 & 8.8 & 1.9 & 1.5 & 4.9 & 5.6 & 1.4 & 9.4 & 9.3 & 2.0 & na & 132 & 4.9 & na & 18.5 \\
\hline $\begin{array}{l}\text { Garnet Canyon, } \\
\text { Wyo. }\end{array}$ & 4/1/1995 & E & -6.7 & 4.0 & 5.12 & 7.6 & $<3.1$ & $<1.7$ & 1.0 & $<0.4$ & 5.4 & $<1.0$ & 5.0 & 7.8 & 0.4 & na & 200 & 5.2 & na & 4.7 \\
\hline $\begin{array}{l}\text { Grand Mesa, } \\
\text { Colo. }\end{array}$ & $3 / 29 / 1995$ & E & -4.0 & 4.1 & 5.25 & 5.6 & 9.5 & $<1.7$ & 1.3 & 0.5 & 4.7 & $<1.0$ & 7.5 & 10.4 & 0.9 & na & 248 & 5.0 & na & 9.5 \\
\hline $\begin{array}{l}\text { Granite Pass, } \\
\text { Mont. }\end{array}$ & 3/17/1995 & E & -4.5 & 2.3 & 5.26 & 5.5 & $<3.1$ & $<1.7$ & $<1.0$ & $<0.4$ & 1.6 & $<1.0$ & 2.3 & 2.4 & 0.5 & na & 200 & 4.0 & na & 20.4 \\
\hline $\begin{array}{l}\text { Gypsum Creek, } \\
\text { Wyo. }\end{array}$ & $3 / 23 / 1995$ & E & -5.9 & 4.5 & 5.13 & 7.4 & 5.0 & 3.3 & 1.3 & 3.6 & 2.9 & 1.4 & 7.7 & 7.8 & 1.8 & na & 82 & 6.3 & na & 16.2 \\
\hline $\begin{array}{l}\text { Hogan Peak, } \\
\text { Colo. }\end{array}$ & 4/8/1995 & E & -15.1 & na & 4.84 & 14.5 & 3.9 & $<1.7$ & 1.8 & 0.8 & 6.3 & $<1.0$ & 12.1 & 10.1 & 0.7 & na & 266 & na & na & 10.1 \\
\hline $\begin{array}{l}\text { Hopewell, } \\
\text { N. Mex. }\end{array}$ & $3 / 26 / 1995$ & E & -2.2 & 3.6 & 5.37 & 4.3 & 7.0 & $<1.7$ & $<1.0$ & 0.8 & 5.1 & $<1.0$ & 6.5 & 7.1 & 0.7 & na & 184 & 3.8 & na & 11.8 \\
\hline $\begin{array}{l}\text { Kings Hill, } \\
\text { Mont. }\end{array}$ & $3 / 15 / 1995$ & E & -1.8 & 3.2 & 5.39 & 4.1 & 5.0 & $<1.7$ & 1.3 & 1.0 & 7.0 & $<1.0$ & 5.6 & 6.6 & 1.1 & na & 130 & 6.4 & na & 20.2 \\
\hline $\begin{array}{l}\text { Lake Irene, } \\
\text { Colo. }\end{array}$ & $3 / 29 / 1995$ & E & -11.7 & 5.6 & 4.97 & 10.7 & 3.1 & $<1.7$ & 1.4 & $<0.4$ & 3.7 & $<1.0$ & 7.1 & 9.6 & 0.6 & na & 150 & 6.1 & na & 6.6 \\
\hline $\begin{array}{c}\text { Lewis Lake } \\
\text { Divide, } \\
\text { Wyo. }\end{array}$ & $3 / 11 / 1995$ & E & -5.5 & 4.1 & 5.13 & 7.4 & $<3.1$ & $<1.7$ & $<1.0$ & 1.0 & 5.9 & 1.1 & 4.8 & 6.6 & 1.0 & na & 216 & 4.3 & na & 6.7 \\
\hline $\begin{array}{l}\text { Lionshead, } \\
\text { Mont. }\end{array}$ & $3 / 13 / 1995$ & E & -6.7 & 4.8 & 5.12 & 7.6 & 3.5 & $<1.7$ & $<1.0$ & 0.8 & 9.7 & 1.4 & 7.5 & 8.5 & 0.7 & na & 195 & 4.5 & na & 10.6 \\
\hline $\begin{array}{l}\text { Lionshead } \\
\text { North, } \\
\text { Mont. }\end{array}$ & $3 / 15 / 1995$ & E & -7.7 & 4.6 & 5.09 & 8.1 & $<3.1$ & $<1.7$ & $<1.0$ & 0.8 & 9.1 & 1.1 & 6.3 & 9.1 & 0.7 & na & 200 & na & na & 4.2 \\
\hline $\begin{array}{l}\text { Loch Vale For- } \\
\text { est, Colo. }\end{array}$ & 4/12/1995 & E & -8.9 & 6.6 & 4.99 & 10.2 & 8.6 & 2.0 & 2.0 & 2.6 & 6.2 & 1.4 & 9.8 & 12.9 & 1.8 & na & 204 & 6.2 & na & 13.5 \\
\hline $\begin{array}{l}\text { Loveland Pass, } \\
\text { Colo. }\end{array}$ & 4/20/1995 & E & -4.2 & 3.4 & 5.25 & 5.6 & 7.5 & $<1.7$ & 2.2 & 0.8 & 3.4 & 1.7 & 5.0 & 8.4 & 1.0 & na & 188 & 4.9 & na & 12.6 \\
\hline $\begin{array}{l}\text { Lynx Pass, } \\
\text { Colo. }\end{array}$ & $3 / 14 / 1995$ & E & -11.2 & 5.5 & 4.99 & 10.2 & 3.9 & $<1.7$ & 1.4 & $<0.4$ & 3.8 & $<1.0$ & 5.8 & 11.7 & 0.7 & na & 128 & 6.3 & na & 4.9 \\
\hline $\begin{array}{l}\text { Molas Lake, } \\
\text { Colo. }\end{array}$ & 4/1/1995 & E & -4.0 & 3.4 & 5.27 & 5.4 & 8.0 & $<1.7$ & $<1.0$ & $<0.4$ & 3.0 & $<1.0$ & 5.4 & 8.9 & 0.6 & na & 213 & 4.6 & na & 6.5 \\
\hline
\end{tabular}


Table 4. Selected chemical and physical data for Rocky Mountain snowpack samples for 1995.—Continued

[E, depth-integrated environmental; VWM, volume-weighted mean of two snowpack layers; ANC, acid neutralization capacity; $\mu$ eq/L, microequivalents per liter; $\mu \mathrm{S} / \mathrm{cm}$, microsiemens per centimeter; SC, specific conductance at $25^{\circ} \mathrm{C} ; \mathrm{pH}$ values in standard units; $\mathrm{H}$, hydrogen; $\mathrm{Ca}$, calcium; $\mathrm{Mg}$, magnesium; $\mathrm{Na}$, sodium; $\mathrm{K}$, potassium; $\mathrm{NH}_{4}$, ammonium; $\mathrm{Cl}$, chloride; $\mathrm{SO}$, sulfate; $\mathrm{NO}{ }_{3}$, nitrate; $\mathrm{DOC}$, dissolved organic carbon; $\mathrm{mg} / \mathrm{L}$, milligrams per liter; SWE, snow/water equivalent; cm, centimeters; $\delta^{34} \mathrm{~S}$, stable sulfur isotope ratio $\left.{ }^{(34} \mathrm{S} /{ }^{32} \mathrm{~S}\right) ; \mathrm{Hg}$, mercury; ng/L, nanograms per liter; na, not analyzed; <, below reporting limits; \%, percent]

\begin{tabular}{|c|c|c|c|c|c|c|c|c|c|c|c|c|c|c|c|c|c|c|c|c|}
\hline Site name & $\begin{array}{l}\text { Sample } \\
\text { date }\end{array}$ & $\begin{array}{l}\text { Sample } \\
\text { type }\end{array}$ & 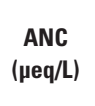 & $\begin{array}{c}\mathrm{SC} \\
(\mu \mathrm{S} / \mathrm{cm})\end{array}$ & $\mathrm{pH}$ & $\underset{\text { ( } \mu \text { eq/L) }}{H}$ & $\underset{(\mu \mathrm{eq} / \mathrm{L})}{\mathrm{Ca}}$ & $\begin{array}{c}\mathrm{Mg} \\
\text { (peq/L) }\end{array}$ & $\underset{\text { (peq/L) }}{\mathrm{Na}}$ & 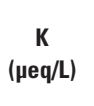 & $\begin{array}{c}\mathrm{NH}_{4} \\
\text { ( } \mu \mathrm{eq} / \mathrm{L} \text { ) }\end{array}$ & 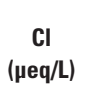 & 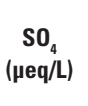 & $\begin{array}{c}\mathrm{NO}_{3} \\
(\mu \mathrm{eq} / \mathrm{L})\end{array}$ & $\begin{array}{c}\text { DOC } \\
\text { (mg/L) }\end{array}$ & $\begin{array}{l}\text { SWE } \\
\text { (cm) }\end{array}$ & $\begin{array}{l}\text { Snow } \\
\text { depth } \\
\text { (cm) }\end{array}$ & $\begin{array}{c}\delta^{34} \mathbf{S} \\
\text { (per mil) }\end{array}$ & $\underset{(\mathrm{ng} / \mathrm{L})}{\mathrm{Hg}}$ & $\begin{array}{c}\text { lonic } \\
\text { balance } \\
(\%)\end{array}$ \\
\hline $\begin{array}{l}\text { Monarch Pass, } \\
\text { Colo. }\end{array}$ & $3 / 31 / 1995$ & $\mathrm{E}$ & 1.0 & 3.5 & 5.64 & 2.3 & 16.4 & $<1.7$ & 1.3 & 0.5 & 4.4 & $<1.0$ & 6.5 & 10.1 & 0.8 & na & 170 & 5.8 & na & 17.2 \\
\hline $\begin{array}{l}\text { Ned Wilson, } \\
\text { Colo. }\end{array}$ & 6/2/1995 & VWM & -0.9 & 3.2 & 5.60 & 2.9 & 9.8 & $<1.7$ & 1.3 & 0.5 & 3.9 & $<1.0$ & 5.8 & 7.2 & 0.9 & 116 & 295 & 5.2 & na & 17.1 \\
\hline $\begin{array}{l}\text { Niwot Snotel, } \\
\text { Colo. }\end{array}$ & 4/3/1995 & E & -9.8 & 5.9 & 5.03 & 9.3 & 7.6 & 1.8 & 1.4 & 1.0 & 8.2 & 1.4 & 9.4 & 15.1 & 1.1 & na & 100 & 6.0 & na & 6.3 \\
\hline $\begin{array}{l}\text { Noisy Basin, } \\
\text { Mont. }\end{array}$ & $3 / 16 / 1995$ & $\mathrm{E}$ & -5.7 & 3.3 & 5.19 & 6.5 & $<3.1$ & $<1.7$ & 1.3 & 0.5 & 4.1 & $<1.0$ & 3.3 & 4.1 & 0.9 & na & 255 & 5.5 & na & 24.6 \\
\hline $\begin{array}{c}\text { Old Battle, } \\
\text { Wyo. }\end{array}$ & $3 / 24 / 1995$ & E & -10.6 & 6.3 & 4.94 & 11.5 & 7.0 & $<1.7$ & 1.3 & 1.5 & 6.3 & 1.4 & 10.8 & 12.1 & 1.1 & na & 210 & 6.3 & na & 6.2 \\
\hline $\begin{array}{l}\text { Old Faithful } \\
\text { Crew Cor- } \\
\text { rals, Wyo. }\end{array}$ & 3/14/1995 & E & -5.6 & 3.6 & 5.21 & 6.2 & $<3.1$ & $<1.7$ & $<1.0$ & $<0.4$ & 5.9 & 1.4 & 3.5 & 7.4 & 0.5 & na & 104 & na & na & -1.2 \\
\hline $\begin{array}{l}\text { Old Faithful } \\
\text { East Lot, } \\
\text { Wyo. }\end{array}$ & $3 / 14 / 1995$ & E & -4.5 & 3.0 & 5.31 & 4.9 & $<3.1$ & $<1.7$ & 2.1 & $<0.4$ & 5.1 & 1.4 & 3.3 & 6.1 & 0.5 & na & 106 & na & na & 5.5 \\
\hline $\begin{array}{l}\text { Old Faithful } \\
\text { Fire Road, } \\
\text { Wyo. }\end{array}$ & $3 / 14 / 1995$ & E & -5.8 & 4.1 & 5.15 & 7.1 & $<3.1$ & $<1.7$ & $<1.0$ & $<0.4$ & 6.2 & 1.4 & 4.8 & 7.3 & 1.0 & na & 114 & na & na & -0.8 \\
\hline $\begin{array}{c}\text { Phantom Val- } \\
\text { ley, Colo. }\end{array}$ & 3/28/1995 & E & -13.7 & 6.5 & 4.89 & 12.9 & 3.8 & $<1.7$ & 1.7 & 0.5 & 4.6 & 1.1 & 8.3 & 11.4 & 1.2 & na & 27 & 5.7 & na & 5.8 \\
\hline $\begin{array}{l}\text { Rabbit Ears 1, } \\
\text { Colo. }\end{array}$ & $3 / 30 / 1995$ & VWM & -13.7 & 7.5 & 4.95 & 13.8 & 5.1 & $<1.7$ & 1.7 & 1.1 & 5.9 & 1.1 & 12.1 & 10.1 & 0.8 & 67 & 210 & 7.5 & na & 8.4 \\
\hline $\begin{array}{l}\text { Rabbit Ears 2, } \\
\text { Colo. }\end{array}$ & 3/30/1995 & VWM & -13.5 & 7.4 & 4.90 & 13.5 & 5.4 & $<1.7$ & 1.1 & 0.6 & 6.4 & $<1.0$ & 12.5 & 11.8 & 0.8 & 65 & 208 & 7.4 & na & 5.2 \\
\hline $\begin{array}{l}\text { Red Mountain, } \\
\text { Mont. }\end{array}$ & $3 / 18 / 1995$ & E & -2.1 & 3.0 & 5.35 & 4.5 & 3.5 & $<1.7$ & 1.7 & $<0.4$ & 5.4 & $<1.0$ & 4.2 & 5.6 & 0.8 & na & 156 & 5.3 & na & 21.3 \\
\hline $\begin{array}{l}\text { Red Mountain } \\
\text { Pass, Colo. }\end{array}$ & 4/1/1995 & E & -2.3 & 3.4 & 5.42 & 3.8 & 7.1 & $<1.7$ & $<1.0$ & 2.1 & 3.1 & 1.1 & 5.4 & 7.9 & 0.9 & na & 228 & 5.0 & na & 5.2 \\
\hline $\begin{array}{l}\text { Rendezvous } \\
\text { Mountain, } \\
\text { Wyo. }\end{array}$ & 4/4/1995 & E & -6.0 & 3.7 & 5.15 & 7.1 & $<3.1$ & $<1.7$ & $<1.0$ & $<0.4$ & 3.6 & $<1.0$ & 4.4 & 5.6 & 0.7 & na & 249 & 5.9 & na & 3.7 \\
\hline $\begin{array}{l}\text { Slumgullion } \\
\text { Pass, Colo. }\end{array}$ & $4 / 2 / 1995$ & $\mathrm{E}$ & -3.0 & 3.2 & 5.32 & 4.8 & 8.0 & $<1.7$ & $<1.0$ & 0.8 & 2.4 & $<1.0$ & 4.6 & 6.5 & 1.0 & na & 130 & 4.4 & na & 17.8 \\
\hline $\begin{array}{l}\text { Snow Bowl, } \\
\text { Mont. }\end{array}$ & $3 / 17 / 1995$ & E & -4.8 & 2.5 & 5.21 & 6.2 & $<3.1$ & $<1.7$ & $<1.0$ & $<0.4$ & 2.9 & $<1.0$ & 3.3 & 3.5 & 0.8 & na & 170 & 4.6 & na & 13.8 \\
\hline
\end{tabular}


Table 4. Selected chemical and physical data for Rocky Mountain snowpack samples for 1995.—Continued

[E, depth-integrated environmental; VWM, volume-weighted mean of two snowpack layers; ANC, acid neutralization capacity; $\mu$ eq/L, microequivalents per liter; $\mu \mathrm{S} / \mathrm{cm}$, microsiemens per centimeter; SC, specific conductance at $25^{\circ} \mathrm{C} ; \mathrm{pH}$ values in standard units; $\mathrm{H}$, hydrogen; $\mathrm{Ca}$, calcium; $\mathrm{Mg}$, magnesium; $\mathrm{Na}$, sodium; $\mathrm{K}$, potassium; $\mathrm{NH}$, ammonium; $\mathrm{Cl}$, chloride; $\mathrm{SO}$, sulfate; $\mathrm{NO}{ }_{3}$, nitrate; $\mathrm{DOC}$, dissolved organic carbon; $\mathrm{mg} / \mathrm{L}$, milligrams per liter; SWE, snow/water equivalent; cm, centimeters; $\delta^{34} \mathrm{~S}$, stable sulfur isotope ratio $\left.{ }^{(34} \mathrm{S} /{ }^{32} \mathrm{~S}\right) ; \mathrm{Hg}$, mercury; ng/L, nanograms per liter; na, not analyzed; <, below reporting limits; \%, percent]

\begin{tabular}{|c|c|c|c|c|c|c|c|c|c|c|c|c|c|c|c|c|c|c|c|c|}
\hline Site name & $\begin{array}{c}\text { Sample } \\
\text { date }\end{array}$ & $\begin{array}{c}\text { Sample } \\
\text { type }\end{array}$ & $\begin{array}{c}\text { ANC } \\
(\mu \mathrm{eq} / \mathrm{L})\end{array}$ & $\begin{array}{c}\text { SC } \\
(\mu \mathrm{S} / \mathrm{cm})\end{array}$ & $\mathrm{pH}$ & $\begin{array}{c}H \\
(\mu e q / L)\end{array}$ & $\begin{array}{c}\text { Ca } \\
(\mu \mathrm{eq} / \mathrm{L})\end{array}$ & $\begin{array}{c}\mathrm{Mg} \\
(\mu \mathrm{eq} / \mathrm{L})\end{array}$ & $\begin{array}{c}\mathrm{Na} \\
(\mu \mathrm{eq} / \mathrm{L})\end{array}$ & $\begin{array}{c}K \\
(\mu \mathrm{eq} / L)\end{array}$ & $\begin{array}{c}\mathbf{N H}_{4} \\
(\mu \mathrm{eq} / \mathrm{L})\end{array}$ & $\begin{array}{c}C I \\
(\mu \mathrm{eq} / \mathrm{L})\end{array}$ & $\underset{(\mu \mathrm{eq} / L)}{\mathrm{SO}_{4}}$ & $\begin{array}{c}\mathrm{NO}_{3} \\
\text { ( } \mu \mathrm{eq} / \mathrm{L} \text { ) }\end{array}$ & $\begin{array}{c}\text { DOC } \\
\text { (mg/L) }\end{array}$ & $\begin{array}{l}\text { SWE } \\
\text { (cm) }\end{array}$ & $\begin{array}{l}\text { Snow } \\
\text { depth } \\
\text { (cm) }\end{array}$ & $\begin{array}{c}\delta^{34} \mathbf{S} \\
\text { (per mil) }\end{array}$ & $\begin{array}{c}\mathrm{Hg} \\
(\mathbf{n g} / \mathrm{L})\end{array}$ & $\begin{array}{c}\text { lonic } \\
\text { balance } \\
(\%)\end{array}$ \\
\hline $\begin{array}{c}\text { South Pass, } \\
\text { Wyo. }\end{array}$ & $3 / 22 / 1995$ & E & -10.2 & 6.1 & 4.98 & 10.5 & 5.5 & $<1.7$ & 2.2 & $<0.4$ & 5.1 & $<1.0$ & 10.8 & 10.9 & 0.9 & na & 149 & 7.3 & na & 3.5 \\
\hline $\begin{array}{l}\text { Sunlight Peak, } \\
\text { Colo. }\end{array}$ & $3 / 29 / 1995$ & E & -5.9 & 3.9 & 5.16 & 6.9 & 6.0 & $<1.7$ & $<1.0$ & $<0.4$ & 4.8 & $<1.0$ & 7.1 & 8.1 & 0.8 & na & 212 & 5.8 & na & 7.7 \\
\hline $\begin{array}{c}\text { Sylvan Lake, } \\
\text { Wyo. }\end{array}$ & $3 / 12 / 1995$ & E & -2.5 & 2.8 & 5.41 & 3.9 & $<3.1$ & $<1.7$ & 1.8 & $<0.4$ & 5.2 & $<1.0$ & 5.0 & 5.0 & 0.9 & na & 198 & 5.0 & na & 4.4 \\
\hline Targee, Mont. & $3 / 15 / 1995$ & E & -5.6 & 4.5 & 5.17 & 6.8 & 3.7 & $<1.7$ & 1.7 & 1.0 & 9.7 & 1.4 & 7.3 & 9.4 & 1.0 & na & 142 & na & na & 11.8 \\
\hline $\begin{array}{c}\text { Teton Pass, } \\
\text { Wyo. }\end{array}$ & $3 / 21 / 1995$ & E & 1.7 & 3.6 & 5.69 & 2.0 & 8.5 & 2.5 & 4.4 & 1.0 & 5.8 & 2.3 & 7.1 & 6.9 & 1.0 & na & 140 & 6.8 & na & 14.9 \\
\hline $\begin{array}{c}\text { Togwotee Pass, } \\
\text { Wyo. }\end{array}$ & $3 / 21 / 1995$ & E & -2.2 & 2.8 & 5.44 & 3.6 & 4.0 & $<1.7$ & 1.7 & $<0.4$ & 3.1 & $<1.0$ & 4.6 & 4.9 & 0.5 & na & 222 & 5.4 & na & 13.6 \\
\hline $\begin{array}{l}\text { Twenty-one } \\
\text { Mile, Mont. }\end{array}$ & 3/13/1995 & E & -6.9 & 4.2 & 5.14 & 7.2 & $<3.1$ & $<1.7$ & 2.6 & $<0.4$ & 5.2 & 1.4 & 5.0 & 7.3 & 0.7 & na & 155 & 4.4 & na & 4.7 \\
\hline $\begin{array}{l}\text { University } \\
\text { Camp, Colo. }\end{array}$ & 4/10/1995 & E & -6.0 & 5.1 & 5.16 & 6.9 & 9.0 & $<1.7$ & 1.3 & 0.8 & 7.6 & $<1.0$ & 9.4 & 11.7 & 1.1 & na & 128 & 5.7 & na & 9.5 \\
\hline $\begin{array}{l}\text { West Yellow- } \\
\text { stone, Mont. }\end{array}$ & $3 / 20 / 1995$ & E & -8.7 & 4.6 & 5.03 & 9.3 & $<3.1$ & $<1.7$ & $<1.0$ & $<0.4$ & 8.6 & 1.7 & 5.6 & 10.9 & 0.9 & na & 107 & 4.4 & na & -1.0 \\
\hline $\begin{array}{l}\text { West Yellow- } \\
\text { stone East, } \\
\text { Mont. }\end{array}$ & $3 / 15 / 1995$ & E & -7.0 & 4.1 & 5.16 & 6.9 & $<3.1$ & $<1.7$ & na & $<0.4$ & 7.4 & 1.7 & 4.4 & 7.6 & 0.6 & na & 104 & na & na & 2.0 \\
\hline $\begin{array}{l}\text { Wolf Creek } \\
\text { Pass, Colo. }\end{array}$ & 4/2/1995 & E & -7.2 & 4.9 & 5.11 & 7.8 & 8.5 & $<1.7$ & $<1.0$ & $<0.4$ & 4.9 & $<1.0$ & 9.2 & 9.6 & 0.8 & na & 288 & 4.5 & na & 6.1 \\
\hline
\end{tabular}


[E, depth-integrated environmental; VWM, volume-weighted mean of two snowpack layers; ANC, acid neutralization capacity; $\mu$ eq/L, microequivalents per liter; $\mu \mathrm{S} / \mathrm{cm}$, microsiemens per centimeter; SC, specific conductance at $25^{\circ} \mathrm{C} ; \mathrm{pH}$ values in standard units; $\mathrm{H}$, hydrogen; $\mathrm{Ca}$, calcium; $\mathrm{Mg}$, magnesium; $\mathrm{Na}$, sodium; $\mathrm{K}$, potassium; $\mathrm{NH}_{4}$, ammonium; $\mathrm{Cl}$, chloride; $\mathrm{SO}$, sulfate; $\mathrm{NO}{ }_{3}$, nitrate; $\mathrm{DOC}$, dissolved organic carbon; $\mathrm{mg} / \mathrm{L}$, milligrams per liter; SWE, snow/water equivalent; cm, centimeters; $\delta^{34} \mathrm{~S}$, stable sulfur isotope ratio $\left.{ }^{34} \mathrm{~S} /{ }^{32} \mathrm{~S}\right) ; \mathrm{Hg}$, mercury; ng/L, nanograms per liter; na, not analyzed; <, below reporting limits; (), suspect ion balance; \%, percent]

\begin{tabular}{|c|c|c|c|c|c|c|c|c|c|c|c|c|c|c|c|c|c|c|c|c|}
\hline Site name & $\begin{array}{c}\text { Sample } \\
\text { date }\end{array}$ & $\begin{array}{c}\text { Sample } \\
\text { type }\end{array}$ & $\begin{array}{c}\text { ANC } \\
\text { ( } \mu \mathrm{eq} / \mathrm{L})\end{array}$ & $\begin{array}{c}\text { SC } \\
(\mu \mathrm{S} / \mathrm{cm})\end{array}$ & $\mathrm{pH}$ & $\begin{array}{c}\mathrm{H} \\
(\mu \mathrm{eq} / \mathrm{L})\end{array}$ & $\begin{array}{c}\text { Ca } \\
\text { (peq/L) }\end{array}$ & $\underset{(\mu \mathrm{eq} / \mathrm{L})}{\mathbf{M g}}$ & $\begin{array}{c}\mathrm{Na} \\
(\mu \mathrm{eq} / \mathrm{L})\end{array}$ & $\begin{array}{c}K \\
\text { (peq/L) }\end{array}$ & $\begin{array}{c}\mathrm{NH}_{4} \\
(\mu \mathrm{eq} / \mathrm{L})\end{array}$ & 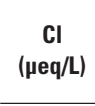 & $\begin{array}{c}\mathrm{SO}_{4} \\
(\mu \mathrm{eq} / \mathrm{L})\end{array}$ & $\begin{array}{c}\mathrm{NO}_{3} \\
\text { ( } \mu \mathrm{eq} / \mathrm{L})\end{array}$ & $\begin{array}{c}\text { DOC } \\
(\mathrm{mg} / \mathrm{L})\end{array}$ & $\begin{array}{l}\text { SWE } \\
\text { (cm) }\end{array}$ & $\begin{array}{l}\text { Snow } \\
\text { depth } \\
\text { (cm) }\end{array}$ & $\begin{array}{c}\delta^{34} \mathbf{S} \\
\text { (per mil) }\end{array}$ & $\begin{array}{c}\mathrm{Hg} \\
(\mathbf{n g} / \mathrm{L})\end{array}$ & $\begin{array}{c}\text { Ionic } \\
\text { balance } \\
(\%)\end{array}$ \\
\hline $\begin{array}{l}\text { Apgar Lookout, } \\
\text { Mont. }\end{array}$ & $3 / 19 / 1996$ & $\mathrm{E}$ & -6.3 & 2.7 & 5.24 & 5.8 & $<3.1$ & $<1.7$ & 4.1 & 3.8 & 1.9 & $<1.0$ & 2.5 & 5.2 & 1.0 & na & 160 & na & na & 33.9 \\
\hline $\begin{array}{l}\text { Berthoud Pass, } \\
\text { Colo. }\end{array}$ & 4/2/1996 & E & -5.6 & 3.8 & 5.16 & 6.9 & 3.6 & $<1.7$ & 2.2 & 0.5 & 1.5 & 1.1 & 4.4 & 6.8 & 0.5 & na & 214 & 6.3 & na & 8.9 \\
\hline $\begin{array}{l}\text { Big Mountain, } \\
\text { Mont. }\end{array}$ & $3 / 15 / 1996$ & $\mathrm{E}$ & -3.1 & 2.5 & 5.23 & 5.9 & $<3.1$ & $<1.7$ & $<1.0$ & 0.6 & 2.1 & $<1.0$ & 2.9 & 3.1 & 0.6 & na & 286 & 6.0 & na & 17.4 \\
\hline Big Sky, Mont. & $3 / 18 / 1996$ & E & 0.2 & 2.5 & 5.57 & 2.7 & 4.3 & $<1.7$ & $<1.0$ & 1.3 & 2.3 & $<1.0$ & 3.3 & 5.2 & 0.5 & na & 257 & 6.3 & na & 9.5 \\
\hline $\begin{array}{l}\text { Biscuit Basin, } \\
\text { Wyo. }\end{array}$ & $3 / 11 / 1996$ & E & 1.4 & 3.3 & 5.64 & 2.3 & 4.0 & $<1.7$ & 1.8 & 2.6 & 4.6 & 2.0 & 2.9 & 5.9 & 1.3 & na & 125 & na & na & 11.2 \\
\hline $\begin{array}{l}\text { Brooklyn Lake, } \\
\text { Wyo. }\end{array}$ & $3 / 24 / 1996$ & E & -5.1 & 4.3 & 5.17 & 6.8 & 5.5 & $<1.7$ & 1.2 & 0.7 & 3.2 & 1.1 & 6.7 & 9.2 & 0.7 & na & 218 & 7.5 & na & 1.1 \\
\hline Brumley, Colo. & $3 / 28 / 1996$ & $\mathrm{E}$ & -1.7 & 3.4 & 5.39 & 4.1 & 7.0 & $<1.7$ & $<1.0$ & 1.5 & 1.6 & $<1.0$ & 4.6 & 6.6 & 0.6 & na & 136 & 6.6 & na & 11.9 \\
\hline $\begin{array}{l}\text { Buffalo Pass, } \\
\text { Colo. }\end{array}$ & 4/4/1996 & E & -10.3 & 5.4 & 5.00 & 10.0 & 5.8 & $<1.7$ & 1.5 & 0.9 & 3.6 & $<1.0$ & 9.0 & 10.3 & 0.6 & na & 300 & 7.6 & na & 6.3 \\
\hline $\begin{array}{l}\text { Cameron Pass, } \\
\text { Colo. }\end{array}$ & 4/3/1996 & E & -4.7 & 3.3 & 5.18 & 6.6 & 4.6 & $<1.7$ & 1.3 & 1.4 & 2.6 & 1.1 & 6.0 & 6.1 & 1.3 & na & 205 & 7.3 & na & 10.9 \\
\hline Canyon, Wyo. & $3 / 10 / 1996$ & E & -1.7 & 2.9 & 5.37 & 4.3 & $<3.1$ & $<1.7$ & 1.0 & 0.8 & 3.4 & $<1.0$ & 2.9 & 4.4 & 0.8 & na & 166 & na & na & 13.0 \\
\hline $\begin{array}{l}\text { Chief Joseph } \\
\text { Pass, Mont. }\end{array}$ & $3 / 17 / 1996$ & E & -4.1 & 2.5 & 5.24 & 5.8 & $<3.1$ & $<1.7$ & $<1.0$ & 0.5 & 1.0 & $<1.0$ & 2.1 & 4.9 & 0.6 & na & 210 & na & na & 2.0 \\
\hline $\begin{array}{l}\text { Daisy Pass, } \\
\text { Mont. }\end{array}$ & $3 / 13 / 1996$ & E & -1.0 & 2.3 & 5.49 & 3.2 & $<3.1$ & $<1.7$ & $<1.0$ & 0.8 & 3.0 & $<1.0$ & 3.5 & 3.9 & 0.5 & na & 318 & 4.0 & na & -2.8 \\
\hline $\begin{array}{l}\text { Deadman Pass, } \\
\text { Colo. }\end{array}$ & 4/9/1996 & E & -3.1 & 3.8 & 5.30 & 5.0 & 8.5 & $<1.7$ & 1.4 & 2.0 & 3.6 & 1.1 & 7.9 & 9.8 & 1.9 & na & 243 & 6.7 & na & 4.2 \\
\hline $\begin{array}{l}\text { Divide Peak, } \\
\text { Wyo. }\end{array}$ & $3 / 23 / 1996$ & E & -3.8 & 4.3 & 5.19 & 6.5 & 8.0 & $<1.7$ & 1.9 & 0.6 & 3.4 & 1.4 & 6.9 & 11.7 & 0.6 & na & 128 & 7.8 & na & 0.5 \\
\hline Dry Lake, Colo. & $3 / 15 / 1996$ & E & -8.8 & 6.2 & 5.00 & 10.0 & 7.0 & $<1.7$ & 1.1 & 2.0 & 3.0 & $<1.0$ & 7.9 & 12.2 & 0.5 & na & 170 & 8.5 & na & 6.6 \\
\hline $\begin{array}{l}\text { Dunckley Pass, } \\
\text { Colo. }\end{array}$ & 4/3/1996 & E & -1.0 & 3.2 & 5.47 & 3.4 & 7.9 & $<1.7$ & 1.2 & 1.0 & 3.4 & $<1.0$ & 5.4 & 7.9 & 0.5 & na & 180 & 7.4 & na & 11.9 \\
\hline Elk River, Colo. & $3 / 15 / 1996$ & $\mathrm{E}$ & -6.2 & 4.6 & 5.14 & 7.2 & 7.5 & $<1.7$ & 1.9 & 0.9 & 1.8 & 1.1 & 6.5 & 14.4 & 0.5 & na & 155 & 8.4 & na & -6.5 \\
\hline $\begin{array}{l}\text { Elkhart Park, } \\
\text { Wyo. }\end{array}$ & 3/21/1996 & E & -2.8 & 3.2 & 5.34 & 4.6 & 3.5 & $<1.7$ & 1.3 & 0.9 & 3.2 & 1.1 & 4.4 & 5.4 & 0.6 & na & 143 & 6.9 & na & 10.1 \\
\hline $\begin{array}{l}\text { Four Mile } \\
\text { Meadow, } \\
\text { Wyo. }\end{array}$ & $3 / 20 / 1996$ & E & -3.0 & 2.4 & 5.46 & 3.5 & $<3.1$ & $<1.7$ & 1.6 & 1.2 & 3.0 & 1.4 & 3.1 & 4.3 & 0.6 & na & 127 & 8.7 & na & 2.4 \\
\hline
\end{tabular}




\section{Table 5. Selected chemical and physical data for Rocky Mountain snowpack samples for 1996.—Continued}

[E, depth-integrated environmental; VWM, volume-weighted mean of two snowpack layers; ANC, acid neutralization capacity; $\mu$ eq/L, microequivalents per liter; $\mu \mathrm{S} / \mathrm{cm}$, microsiemens per centimeter; SC, specific conductance at $25^{\circ} \mathrm{C} ; \mathrm{pH}$ values in standard units; $\mathrm{H}$, hydrogen; $\mathrm{Ca}$, calcium; $\mathrm{Mg}$, magnesium; $\mathrm{Na}$, sodium; $\mathrm{K}$, potassium; $\mathrm{NH}_{4}, \mathrm{ammonium} \mathrm{Cl}_{\text {, chloride; }} \mathrm{SO}$, sulfate; $\mathrm{NO}_{3}$, nitrate; $\mathrm{DOC}$, dissolved organic carbon; $\mathrm{mg} / \mathrm{L}$, milligrams per liter; SWE, snow/water equivalent; cm, centimeters; $\delta^{34} \mathrm{~S}$, stable sulfur isotope ratio $\left.{ }^{34} \mathrm{~S} /{ }^{32} \mathrm{~S}\right) ; \mathrm{Hg}$, mercury; ng/L, nanograms per liter; na, not analyzed; <, below reporting limits; (), suspect ion balance; \%, percent]

\begin{tabular}{|c|c|c|c|c|c|c|c|c|c|c|c|c|c|c|c|c|c|c|c|c|}
\hline Site name & $\begin{array}{c}\text { Sample } \\
\text { date }\end{array}$ & $\begin{array}{c}\text { Sample } \\
\text { type }\end{array}$ & $\begin{array}{c}\text { ANC } \\
\text { ( } \mu \mathrm{eq} / \mathrm{L})\end{array}$ & $\begin{array}{c}\text { SC } \\
(\mu \mathrm{S} / \mathrm{cm})\end{array}$ & $\mathrm{pH}$ & $\begin{array}{c}\mathrm{H} \\
(\mu \mathrm{eq} / \mathrm{L})\end{array}$ & $\begin{array}{c}\mathrm{Ca} \\
(\mu \mathrm{eq} / \mathrm{L})\end{array}$ & $\begin{array}{c}\mathrm{Mg} \\
\text { (peq/L) }\end{array}$ & $\begin{array}{c}\mathrm{Na} \\
(\mu \mathrm{eq} / \mathrm{L})\end{array}$ & 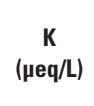 & $\begin{array}{c}\mathrm{NH}_{4} \\
\text { ( } \mu \mathrm{eq} / \mathrm{L} \text { ) }\end{array}$ & 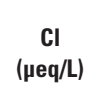 & $\begin{array}{c}\mathrm{SO}_{4} \\
(\mu \mathrm{eq} / \mathrm{L})\end{array}$ & $\begin{array}{c}\mathrm{NO}_{3} \\
(\mu \mathrm{eq} / \mathrm{L})\end{array}$ & $\begin{array}{l}\text { DOC } \\
\text { (mg/L) }\end{array}$ & $\begin{array}{l}\text { SWE } \\
\text { (cm) }\end{array}$ & $\begin{array}{l}\text { Snow } \\
\text { depth } \\
\text { (cm) }\end{array}$ & $\begin{array}{c}\delta^{34} \mathrm{~S} \\
\text { (per mil) }\end{array}$ & $\begin{array}{c}\mathrm{Hg} \\
(\mathbf{n g} / \mathrm{L})\end{array}$ & $\begin{array}{c}\text { lonic } \\
\text { balance } \\
(\%)\end{array}$ \\
\hline $\begin{array}{l}\text { Fremont Pass, } \\
\text { Colo. }\end{array}$ & $4 / 25 / 1996$ & $\mathrm{E}$ & -1.6 & 3.1 & 5.45 & 3.5 & 6.3 & $<1.7$ & 1.1 & 0.8 & 2.1 & $<1.0$ & 4.6 & 6.5 & 0.6 & na & 227 & na & na & 11.2 \\
\hline $\begin{array}{l}\text { Gallegos Peak, } \\
\text { N. Mex. }\end{array}$ & $3 / 26 / 1996$ & E & 19.6 & 5.5 & 6.33 & 0.5 & 34.4 & 6.6 & 1.9 & 2.1 & 4.2 & 1.1 & 9.4 & 10.9 & 1.2 & na & 115 & 4.6 & na & 9.7 \\
\hline $\begin{array}{l}\text { Garnet Canyon, } \\
\text { Wyo. }\end{array}$ & $3 / 14 / 1996$ & E & -4.9 & 2.4 & 5.43 & 3.7 & $<3.1$ & $<1.7$ & 1.3 & 1.9 & 1.9 & $<1.0$ & 3.8 & 3.7 & 0.4 & na & 290 & na & na & 8.7 \\
\hline $\begin{array}{l}\text { Grand Mesa, } \\
\text { Colo. }\end{array}$ & $3 / 29 / 1996$ & E & 0.4 & 3.2 & 5.57 & 2.7 & 9.8 & $<1.7$ & 1.0 & 0.8 & 3.7 & $<1.0$ & 6.5 & 7.6 & 0.7 & na & 166 & 5.4 & na & 11.0 \\
\hline $\begin{array}{l}\text { Granite Pass, } \\
\text { Mont. }\end{array}$ & $3 / 16 / 1996$ & E & -2.8 & 1.9 & 5.37 & 4.3 & $<3.1$ & $<1.7$ & $<1.0$ & 1.4 & $<1.0$ & $<1.0$ & 1.9 & 1.8 & 0.8 & na & 217 & na & na & 21.6 \\
\hline $\begin{array}{l}\text { Gypsum Creek, } \\
\text { Wyo. }\end{array}$ & $3 / 21 / 1996$ & E & -5.8 & 2.4 & 5.23 & 5.9 & $<3.1$ & $<1.7$ & $<1.0$ & $<0.4$ & 2.1 & $<1.0$ & 3.3 & 4.5 & 0.4 & na & 147 & 5.5 & na & 0.8 \\
\hline $\begin{array}{l}\text { Hogan Peak, } \\
\text { Colo. }\end{array}$ & 4/6/1996 & E & -6.8 & 4.5 & 5.18 & 6.6 & 7.0 & $<1.7$ & 1.1 & 1.5 & 5.1 & $<1.0$ & 9.8 & 8.7 & 0.8 & na & 304 & na & na & 7.0 \\
\hline $\begin{array}{l}\text { Hopewell, } \\
\text { N. Mex. }\end{array}$ & $3 / 25 / 1996$ & E & 17.2 & 6.2 & 6.25 & 0.6 & 31.4 & 4.1 & 2.4 & 3.9 & 6.3 & 1.4 & 11.0 & 20.1 & 1.8 & na & 105 & 4.8 & na & -1.1 \\
\hline $\begin{array}{l}\text { Kings Hill, } \\
\text { Mont. }\end{array}$ & $3 / 14 / 1996$ & E & -5.0 & 3.1 & 5.26 & 5.5 & $<3.1$ & $<1.7$ & $<1.0$ & $<0.4$ & 3.1 & $<1.0$ & 4.2 & 5.1 & 0.5 & na & 137 & 7.4 & na & -3.8 \\
\hline Lake Irene, Colo. & 4/11/1996 & E & -5.9 & 3.4 & 5.15 & 7.1 & 3.1 & $<1.7$ & 2.2 & 1.2 & 2.0 & $<1.0$ & 5.8 & 5.4 & 0.8 & na & 170 & 6.7 & na & 16.6 \\
\hline $\begin{array}{l}\text { Lewis Lake } \\
\text { Divide, Wyo. }\end{array}$ & 3/9/1996 & E & -0.8 & 2.5 & 5.52 & 3.0 & 3.2 & $<1.7$ & 1.4 & $<0.4$ & 4.6 & 1.1 & 3.8 & 5.0 & 0.5 & na & 352 & 5.9 & na & 10.5 \\
\hline Lionshead, Mont. & $3 / 11 / 1996$ & $\mathrm{E}$ & 0.6 & 3.9 & 5.55 & 2.8 & 5.2 & $<1.7$ & 2.1 & 4.4 & 9.8 & 2.0 & 6.9 & 9.1 & 1.7 & na & 169 & 5.8 & na & 13.1 \\
\hline $\begin{array}{l}\text { Loch Vale } \\
\text { Meadow, } \\
\text { Colo. }\end{array}$ & 4/9/1996 & E & -8.5 & 4.4 & 5.14 & 7.2 & 4.5 & $<1.7$ & 1.5 & $<0.4$ & 2.2 & 1.1 & 6.0 & 8.0 & 0.5 & 85 & 210 & 7.4 & na & 0.8 \\
\hline $\begin{array}{l}\text { Loveland Pass, } \\
\text { Colo. }\end{array}$ & $5 / 2 / 1996$ & E & -2.3 & 3.0 & 5.42 & 3.8 & 7.0 & $<1.7$ & 2.0 & $<0.4$ & 1.6 & 1.7 & 5.0 & 6.9 & 0.5 & na & 292 & 7.1 & na & 3.0 \\
\hline Lynx Pass, Colo. & $3 / 14 / 1996$ & $\mathrm{E}$ & -9.6 & 4.2 & 5.14 & 7.2 & 4.0 & $<1.7$ & $<1.0$ & 0.4 & 1.4 & $<1.0$ & 4.6 & 8.0 & 0.4 & na & 179 & 7.4 & na & 1.9 \\
\hline $\begin{array}{l}\text { Molas Lake, } \\
\text { Colo. }\end{array}$ & 3/29/1996 & E & 19.0 & 4.3 & 6.44 & 0.4 & 24.0 & 2.5 & 1.6 & 1.2 & 3.4 & $<1.0$ & 5.4 & 7.2 & 0.6 & na & 162 & 3.8 & na & 2.0 \\
\hline $\begin{array}{l}\text { Monarch Pass, } \\
\text { Colo. }\end{array}$ & $3 / 29 / 1996$ & $\mathrm{E}$ & 3.5 & 2.9 & 5.85 & 1.4 & 11.6 & 2.3 & 1.7 & na & 2.3 & $<1.0$ & 6.0 & 7.3 & 0.7 & na & 186 & 6.3 & na & 6.9 \\
\hline $\begin{array}{l}\text { Niwot Snotel, } \\
\text { Colo. }\end{array}$ & 4/9/1996 & $\mathrm{E}$ & -9.7 & 4.6 & 5.18 & 6.6 & 5.5 & $<1.7$ & 1.5 & 1.3 & 4.4 & $<1.0$ & 8.1 & 8.5 & 0.7 & na & 160 & na & na & 7.5 \\
\hline $\begin{array}{l}\text { Noisy Basin, } \\
\text { Mont. }\end{array}$ & $3 / 15 / 1996$ & E & -3.4 & 2.5 & 5.25 & 5.6 & $<3.1$ & $<1.7$ & 1.3 & 9.7 & 2.8 & $<1.0$ & 3.1 & 4.2 & 0.5 & na & 230 & 6.3 & na & 45.2 \\
\hline
\end{tabular}


[E, depth-integrated environmental; VWM, volume-weighted mean of two snowpack layers; ANC, acid neutralization capacity; $\mu$ eq/L, microequivalents per liter; $\mu \mathrm{S} / \mathrm{cm}$, microsiemens per centimeter; SC, specific conductance at $25^{\circ} \mathrm{C} ; \mathrm{pH}$ values in standard units; $\mathrm{H}$, hydrogen; $\mathrm{Ca}$, calcium; $\mathrm{Mg}$, magnesium; $\mathrm{Na}$, sodium; $\mathrm{K}$, potassium; $\mathrm{NH}_{4}$, ammonium; $\mathrm{Cl}$, chloride; $\mathrm{SO}$, sulfate; $\mathrm{NO}{ }_{3}$, nitrate; $\mathrm{DOC}$, dissolved organic carbon; $\mathrm{mg} / \mathrm{L}$, milligrams per liter; SWE, snow/water equivalent; cm, centimeters; $\delta^{34} \mathrm{~S}$, stable sulfur isotope ratio $\left.{ }^{34} \mathrm{~S} /{ }^{32} \mathrm{~S}\right) ; \mathrm{Hg}$, mercury; ng/L, nanograms per liter; na, not analyzed; <, below reporting limits; (), suspect ion balance; \%, percent]

\begin{tabular}{|c|c|c|c|c|c|c|c|c|c|c|c|c|c|c|c|c|c|c|c|c|}
\hline Site name & $\begin{array}{l}\text { Sample } \\
\text { date }\end{array}$ & $\begin{array}{l}\text { Sample } \\
\text { type }\end{array}$ & $\begin{array}{c}\text { ANC } \\
(\mu e q / L)\end{array}$ & $\begin{array}{c}\mathrm{SC} \\
(\mu \mathrm{S} / \mathrm{cm})\end{array}$ & $\mathrm{pH}$ & $\begin{array}{c}H \\
(\mu \mathrm{eq} / \mathrm{L})\end{array}$ & $\begin{array}{c}\text { Ca } \\
(\mu \mathrm{eq} / \mathrm{L})\end{array}$ & $\begin{array}{c}\mathrm{Mg} \\
(\mu \mathrm{eq} / \mathrm{L})\end{array}$ & $\begin{array}{c}\mathrm{Na} \\
(\mu \mathrm{eq} / \mathrm{L})\end{array}$ & $\begin{array}{c}K \\
(\mu \mathrm{eq} / \mathrm{L})\end{array}$ & $\begin{array}{c}\mathrm{NH}_{4} \\
(\mu \mathrm{eq} / \mathrm{L})\end{array}$ & $\begin{array}{c}\text { CI } \\
(\mu \mathrm{eq} / \mathrm{L})\end{array}$ & $\begin{array}{c}\mathrm{SO}_{4} \\
(\mu \mathrm{eq} / \mathrm{L})\end{array}$ & $\begin{array}{c}\mathrm{NO}_{3} \\
\text { ( } \mu \mathrm{eq} / \mathrm{L})\end{array}$ & $\begin{array}{l}\text { DOC } \\
\text { (mg/L) }\end{array}$ & $\begin{array}{l}\text { SWE } \\
(\mathrm{cm})\end{array}$ & $\begin{array}{l}\text { Snow } \\
\text { depth } \\
\text { (cm) }\end{array}$ & $\begin{array}{c}\delta^{34} \mathbf{S} \\
\text { (per mil) }\end{array}$ & $\begin{array}{c}\mathrm{Hg} \\
\text { (ng/L) }\end{array}$ & $\begin{array}{c}\text { lonic } \\
\text { balance } \\
(\%)\end{array}$ \\
\hline Old Battle, Wyo. & $3 / 23 / 1996$ & $\mathrm{E}$ & -4.8 & 4.2 & 5.22 & 6.0 & 7.3 & $<1.7$ & 2.0 & 0.9 & 3.3 & 1.1 & 7.3 & 9.0 & 0.8 & na & 220 & 8.1 & na & 5.6 \\
\hline $\begin{array}{l}\text { Old Faithful } \\
\text { Crew Corrals, } \\
\text { Wyo. }\end{array}$ & $3 / 11 / 1996$ & E & -1.6 & 2.9 & 5.48 & 3.3 & $<3.1$ & $<1.7$ & 10.4 & 3.8 & 4.4 & 1.4 & 3.1 & 6.5 & 0.5 & na & 140 & na & na & 32.9 \\
\hline $\begin{array}{l}\text { Old Faithful East } \\
\text { Lot, Wyo. }\end{array}$ & 3/11/1996 & E & -3.1 & 3.7 & 5.35 & 4.5 & 4.0 & $<1.7$ & 3.3 & 1.6 & 5.4 & 2.8 & 3.8 & 10.4 & 1.5 & na & 130 & na & na & 4.6 \\
\hline $\begin{array}{l}\text { Old Faithful Fire } \\
\text { Road, Wyo. }\end{array}$ & $3 / 11 / 1996$ & $\mathrm{E}$ & -1.5 & 3.4 & 5.37 & 4.3 & 4.0 & $<1.7$ & 2.3 & 1.6 & 5.2 & 2.0 & 4.0 & 8.4 & 1.3 & na & 120 & na & na & 9.6 \\
\hline $\begin{array}{l}\text { Old Faithful (in } \\
\text { road), Wyo. }\end{array}$ & $3 / 11 / 1996$ & E & 1.1 & 4.3 & 5.57 & 2.7 & 11.0 & 2.5 & 2.7 & 8.5 & 7.2 & 2.5 & 6.3 & 8.4 & 2.1 & na & 70 & na & na & $(30.8)$ \\
\hline $\begin{array}{l}\text { Phantom Valley, } \\
\text { Colo. }\end{array}$ & 4/10/1996 & E & -7.2 & 4.5 & 5.15 & 7.1 & 5.0 & $<1.7$ & 1.4 & 1.3 & 2.6 & 1.1 & 6.7 & 7.4 & 0.9 & na & 107 & 7.2 & na & 7.0 \\
\hline $\begin{array}{l}\text { Rabbit Ears 1, } \\
\text { Colo. }\end{array}$ & 4/2/1996 & E & -8.7 & 5.2 & 5.01 & 9.8 & 5.0 & $<1.7$ & 1.1 & 0.9 & 3.0 & $<1.0$ & 7.9 & 8.0 & 1.6 & na & 292 & 7.9 & na & 10.9 \\
\hline $\begin{array}{l}\text { Rabbit Ears 2, } \\
\text { Colo. }\end{array}$ & 4/2/1996 & $\mathrm{E}$ & -9.0 & 4.3 & 5.10 & 7.9 & 5.0 & $<1.7$ & 1.1 & 1.3 & 3.1 & 1.1 & 8.3 & 8.8 & 0.7 & 104 & 285 & 7.7 & na & 0.8 \\
\hline $\begin{array}{l}\text { Red Mountain, } \\
\text { Mont. }\end{array}$ & $3 / 17 / 1996$ & $\mathrm{E}$ & -2.4 & 2.3 & 5.45 & 3.5 & $<3.1$ & $<1.7$ & $<1.0$ & 0.7 & 1.6 & $<1.0$ & 2.7 & 4.1 & 0.6 & na & 108 & 5.8 & na & -7.3 \\
\hline $\begin{array}{r}\text { Red Mountain } \\
\text { Pass, Colo. }\end{array}$ & $3 / 29 / 1996$ & E & 12.0 & 4.3 & 6.27 & 0.5 & 22.0 & 2.5 & 1.7 & 0.9 & 2.9 & 1.7 & 6.0 & 7.9 & 0.8 & na & 173 & 5.2 & na & 4.8 \\
\hline $\begin{array}{l}\text { Rendezvous } \\
\text { Mountain, } \\
\text { Wyo. }\end{array}$ & $3 / 19 / 1996$ & $\mathrm{E}$ & -2.8 & 2.2 & 5.53 & 3.0 & $<3.1$ & $<1.7$ & 1.3 & 0.9 & 2.5 & $<1.0$ & 3.1 & 3.0 & 0.4 & na & 254 & 5.8 & na & 11.0 \\
\hline $\begin{array}{l}\text { Ripple Creek } \\
\text { Pass, Colo. }\end{array}$ & 4/3/1996 & $\mathrm{E}$ & -3.4 & 3.1 & 5.44 & 3.6 & 6.8 & $<1.7$ & 1.3 & 1.1 & 2.9 & $<1.0$ & 5.2 & 6.9 & 0.7 & na & 261 & 7.3 & na & 12.8 \\
\hline $\begin{array}{l}\text { Slumgullion } \\
\text { Pass, Colo. }\end{array}$ & $3 / 28 / 1996$ & $\mathrm{E}$ & 9.8 & 3.5 & 6.11 & 0.8 & 18.5 & $<1.7$ & 1.8 & 1.2 & 2.0 & 1.4 & 5.4 & 6.3 & 0.9 & na & 113 & 3.5 & na & 2.8 \\
\hline $\begin{array}{l}\text { Snow Bowl, } \\
\text { Mont. }\end{array}$ & $3 / 16 / 1996$ & $\mathrm{E}$ & -3.6 & 2.2 & 5.32 & 4.8 & $<3.1$ & $<1.7$ & $<1.0$ & 0.9 & 1.6 & $<1.0$ & 2.7 & 3.1 & 1.3 & na & 231 & 5.2 & na & 11.5 \\
\hline South Pass, Wyo. & $3 / 22 / 1996$ & $\mathrm{E}$ & 8.5 & 3.8 & 5.62 & 2.4 & 7.5 & $<1.7$ & 2.9 & 2.6 & 5.2 & 1.7 & 7.7 & 7.9 & 1.1 & na & 152 & 9.6 & na & -11.3 \\
\hline $\begin{array}{l}\text { Sunlight Peak, } \\
\text { Colo. }\end{array}$ & 4/2/1996 & E & -0.8 & 3.9 & 5.47 & 3.4 & 12.8 & 2.3 & 1.5 & 2.0 & 4.2 & 1.1 & 6.9 & 10.9 & 0.8 & na & 203 & 6.6 & na & 16.3 \\
\hline $\begin{array}{l}\text { Sylvan Lake, } \\
\text { Wyo. }\end{array}$ & $3 / 10 / 1996$ & E & -1.0 & 2.4 & 5.50 & 3.2 & $<3.1$ & $<1.7$ & 1.5 & 1.6 & 3.0 & 1.1 & 3.3 & 3.9 & 0.7 & na & 225 & 4.9 & na & 4.8 \\
\hline $\begin{array}{l}\text { Sylvan Lake (in } \\
\text { road), Wyo. }\end{array}$ & 3/10/1996 & E & -4.7 & 2.6 & 5.41 & 3.9 & $<3.1$ & $<1.7$ & 1.4 & 4.9 & 3.5 & 1.1 & 4.0 & 4.1 & 0.6 & na & 173 & na & na & 19.6 \\
\hline
\end{tabular}




\section{Table 5. Selected chemical and physical data for Rocky Mountain snowpack samples for 1996.—Continued}

[E, depth-integrated environmental; VWM, volume-weighted mean of two snowpack layers; ANC, acid neutralization capacity; ueq/L, microequivalents per liter; $u$ S/cm, microsiemens per centimeter; SC, specific conductance at $25^{\circ} \mathrm{C} ; \mathrm{pH}$ values in standard units; $\mathrm{H}$, hydrogen; $\mathrm{Ca}$, calcium; $\mathrm{Mg}$, magnesium; $\mathrm{Na}$, sodium; $\mathrm{K}$, potassium; $\mathrm{NH}_{4}$, ammonium; $\mathrm{Cl}$, chloride; $\mathrm{SO}$, sulfate; $\mathrm{NO}{ }_{3}$, nitrate; $\mathrm{DOC}$, dissolved organic carbon; mg/L, milligrams per liter; SWE, snow/water equivalent; cm, centimeters; $\delta^{34} \mathrm{~S}$, stable sulfur isotope ratio $\left({ }^{34} \mathrm{~S} /{ }^{32} \mathrm{~S}\right)$; $\mathrm{Hg}$, mercury; ng/L, nanograms per liter; na, not analyzed; <, below reporting limits; (), suspect ion balance; \%, percent]

\begin{tabular}{|c|c|c|c|c|c|c|c|c|c|c|c|c|c|c|c|c|c|c|c|c|}
\hline Site name & $\begin{array}{l}\text { Sample } \\
\text { date }\end{array}$ & $\begin{array}{l}\text { Sample } \\
\text { type }\end{array}$ & $\begin{array}{c}\text { ANC } \\
\text { ( } \mu \mathrm{eq} / \mathrm{L})\end{array}$ & $\begin{array}{c}\mathrm{SC} \\
(\mu \mathrm{S} / \mathrm{cm})\end{array}$ & $\mathrm{pH}$ & 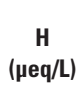 & $\begin{array}{c}\mathrm{Ca} \\
(\mu \mathrm{eq} / \mathrm{L})\end{array}$ & $\underset{(\mu \mathrm{g} / \mathrm{L})}{\mathrm{Mg}}$ & $\begin{array}{c}\mathrm{Na} \\
(\mu \mathrm{eq} / \mathrm{L})\end{array}$ & 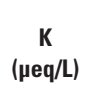 & $\begin{array}{c}\mathrm{NH}_{4} \\
(\mu \mathrm{eq} / \mathrm{L})\end{array}$ & $\underset{(\mu e q / L)}{C l}$ & $\begin{array}{c}\mathrm{SO}_{4} \\
(\mu \mathrm{eq} / \mathrm{L})\end{array}$ & $\begin{array}{c}\mathrm{NO}_{3} \\
(\mu \mathrm{eq} / \mathrm{L})\end{array}$ & $\begin{array}{l}\text { DOC } \\
(\mathrm{mg} / \mathrm{L})\end{array}$ & $\begin{array}{l}\text { SWE } \\
\text { (cm) }\end{array}$ & $\begin{array}{l}\text { Snow } \\
\text { depth } \\
\text { (cm) }\end{array}$ & $\begin{array}{c}\delta^{34} \mathbf{S} \\
\text { (per mil) }\end{array}$ & $\underset{(\mathrm{ng} / \mathrm{L})}{\mathrm{Hg}}$ & $\begin{array}{c}\text { lonic } \\
\text { balance } \\
(\%)\end{array}$ \\
\hline Teton Pass, Wyo. & 3/9/1996 & E & 6.2 & 3.2 & 5.93 & 1.2 & 8.2 & 2.7 & 2.8 & 2.5 & 4.1 & 2.3 & 4.6 & 4.9 & 0.9 & na & 189 & 6.6 & na & 9.2 \\
\hline $\begin{array}{l}\text { Togwotee Pass, } \\
\text { Wyo. }\end{array}$ & $3 / 20 / 1996$ & E & -0.1 & 2.4 & 5.54 & 2.9 & 5.3 & $<1.7$ & 1.6 & 1.3 & 2.5 & 1.1 & 3.5 & 4.2 & 0.7 & na & 255 & 7.0 & na & 21.1 \\
\hline $\begin{array}{l}\text { Trappers Lake, } \\
\text { Colo. }\end{array}$ & 4/3/1996 & E & -2.6 & 3.1 & 5.46 & 3.5 & 6.6 & $<1.7$ & 1.2 & 0.6 & 2.2 & $<1.0$ & 4.6 & 7.0 & 0.6 & na & 189 & 7.0 & na & 9.7 \\
\hline $\begin{array}{l}\text { Twenty-one } \\
\text { Mile, Mont. }\end{array}$ & $3 / 12 / 1996$ & E & 0.3 & 2.9 & 5.55 & 2.8 & 4.7 & $<1.7$ & 1.5 & 1.6 & 4.6 & 1.7 & 3.5 & 5.9 & 1.0 & na & 141 & 6.2 & na & 13.5 \\
\hline $\begin{array}{l}\text { University Camp, } \\
\text { Colo. }\end{array}$ & $4 / 12 / 1996$ & E & -4.3 & 4.0 & 5.15 & 7.1 & 5.5 & $<1.7$ & 1.0 & 3.2 & 4.0 & $<1.0$ & 7.5 & 7.6 & 0.8 & na & 200 & 7.5 & na & 16.0 \\
\hline $\begin{array}{l}\text { West Yellow- } \\
\text { stone, Mont. }\end{array}$ & $3 / 11 / 1996$ & E & -2.2 & 3.3 & 5.39 & 4.1 & 4.0 & $<1.7$ & 1.7 & 4.1 & 5.1 & 2.0 & 4.2 & 7.9 & 1.1 & na & 88 & 6.0 & na & 15.2 \\
\hline $\begin{array}{l}\text { West Yellow- } \\
\text { stone (in } \\
\text { road), Mont. }\end{array}$ & $3 / 12 / 1996$ & E & 5.9 & 5.8 & 5.71 & 1.9 & 13.5 & 2.5 & 9.7 & 5.5 & 8.9 & 11.0 & 8.8 & 7.9 & na & na & 56 & na & na & 11.1 \\
\hline $\begin{array}{l}\text { Wolf Creek Pass, } \\
\text { Colo. }\end{array}$ & $3 / 30 / 1996$ & E & 9.3 & 5.4 & 6.13 & 0.7 & 27.8 & 2.6 & 3.5 & 1.3 & 3.5 & 2.5 & 12.3 & 12.7 & 0.6 & na & 196 & 6.0 & na & 3.5 \\
\hline
\end{tabular}


[E, depth-integrated environmental; VWM, volume-weighted mean of two snowpack layers; ANC, acid neutralization capacity; $\mu$ eq/L, microequivalents per liter; $\mu \mathrm{S} / \mathrm{cm}$, microsiemens per centimeter; SC, specific conductance at $25^{\circ} \mathrm{C} ; \mathrm{pH}$ values in standard units; $\mathrm{H}$, hydrogen; $\mathrm{Ca}$, calcium; $\mathrm{Mg}$, magnesium; $\mathrm{Na}$, sodium; $\mathrm{K}$, potassium; $\mathrm{NH}_{4}$, ammonium; $\mathrm{Cl}$, chloride; $\mathrm{SO}_{4}$, sulfate; $\mathrm{NO}{ }_{3}$, nitrate; $\mathrm{DOC}$, dissolved organic carbon; $\mathrm{mg} / \mathrm{L}$, milligrams per liter; SWE, snow/water equivalent; cm, centimeters; $\delta^{34} \mathrm{~S}$, stable sulfur isotope ratio $\left.{ }^{34} \mathrm{~S} /{ }^{32} \mathrm{~S}\right) ; \mathrm{Hg}$, mercury; ng/L, nanograms per liter; na, not analyzed; <, below reporting limits; (), suspect ion balance; \%, percent]

\begin{tabular}{|c|c|c|c|c|c|c|c|c|c|c|c|c|c|c|c|c|c|c|c|c|}
\hline Site name & $\begin{array}{l}\text { Sample } \\
\text { date }\end{array}$ & $\begin{array}{l}\text { Sample } \\
\text { type }\end{array}$ & $\begin{array}{c}\text { ANC } \\
(\mu \mathrm{eq} / \mathrm{L})\end{array}$ & $\begin{array}{c}\mathrm{SC} \\
(\mu \mathrm{S} / \mathrm{cm})\end{array}$ & $\mathrm{pH}$ & $\begin{array}{c}H \\
(\mu \mathrm{eq} / \mathrm{L})\end{array}$ & $\begin{array}{c}\text { Ca } \\
(\mu \mathrm{eq} / \mathrm{L})\end{array}$ & $\underset{(\mu \mathrm{eq} / \mathrm{L})}{\mathrm{Mg}}$ & $\begin{array}{c}\mathrm{Na} \\
(\mu \mathrm{eq} / \mathrm{L})\end{array}$ & $\begin{array}{c}K \\
\text { (peq/L) }\end{array}$ & $\underset{(\mu \mathrm{eq} / \mathrm{L})}{\mathrm{NH}_{4}}$ & $\begin{array}{c}C I \\
(\mu e q / L)\end{array}$ & 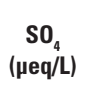 & $\begin{array}{c}\mathrm{NO}_{3} \\
(\mu \mathrm{eq} / \mathrm{L})\end{array}$ & $\begin{array}{l}\text { DOC } \\
(\mu \mathrm{g} / \mathrm{L})\end{array}$ & $\begin{array}{l}\text { SWE } \\
\text { (cm) }\end{array}$ & $\begin{array}{l}\text { Snow } \\
\text { depth } \\
\text { (cm) }\end{array}$ & $\begin{array}{l}\delta^{34} S \\
\text { (per } \\
\text { mil) }\end{array}$ & $\underset{\text { (ng/L) }}{\mathrm{Hg}}$ & $\begin{array}{c}\text { lonic } \\
\text { balance } \\
(\%)\end{array}$ \\
\hline $\begin{array}{l}\text { Apgar Lookout, } \\
\text { Mont. }\end{array}$ & 4/4/1997 & E & -8.0 & 4.3 & 5.01 & 9.8 & $<3.1$ & $<1.7$ & 1.6 & 0.9 & 2.3 & 1.4 & 5.2 & 4.9 & 1.1 & na & 255 & 6.6 & na & 11.5 \\
\hline $\begin{array}{l}\text { Beaver Mountain, } \\
\text { Utah }\end{array}$ & $3 / 25 / 1997$ & E & -4.1 & 4.1 & 5.23 & 5.9 & 7.5 & $<1.7$ & 3.6 & 0.4 & 4.3 & 3.4 & 5.8 & 7.1 & 0.9 & na & 204 & 8.3 & na & 14.0 \\
\hline $\begin{array}{l}\text { Berthoud Pass, } \\
\text { Colo. }\end{array}$ & $4 / 22 / 1997$ & E & -5.4 & 4.0 & 5.14 & 7.2 & 5.7 & $<1.7$ & 1.8 & 0.9 & 3.1 & 1.7 & 5.4 & 7.9 & 0.9 & na & 220 & 5.7 & na & 11.2 \\
\hline $\begin{array}{l}\text { Big Mountain, } \\
\text { Mont. }\end{array}$ & $3 / 16 / 1997$ & E & -9.5 & 3.8 & 5.05 & 8.9 & $<3.1$ & $<1.7$ & $<1.0$ & $<0.4$ & 2.0 & $<1.0$ & 3.7 & 4.6 & na & na & 365 & 6.7 & na & 13.1 \\
\hline Big Sky, Mont. & $3 / 20 / 1997$ & E & -3.9 & 2.2 & 5.38 & 4.2 & $<3.1$ & $<1.7$ & $<1.0$ & $<0.4$ & 2.4 & $<1.0$ & 2.1 & 3.4 & 0.6 & na & 188 & 5.9 & na & 9.0 \\
\hline $\begin{array}{l}\text { Biscuit Basin, } \\
\text { Wyo. }\end{array}$ & $3 / 11 / 1997$ & E & -5.5 & 2.9 & 5.14 & 7.2 & $<3.1$ & $<1.7$ & $<1.0$ & $<0.4$ & 2.4 & $<1.0$ & 2.3 & 4.6 & 1.0 & na & 174 & na & na & 16.7 \\
\hline $\begin{array}{l}\text { Brooklyn Lake, } \\
\text { Wyo. }\end{array}$ & $3 / 25 / 1997$ & E & -11.6 & 6.0 & 4.85 & 14.1 & 5.2 & $<1.7$ & $<1.0$ & 0.9 & 2.7 & $<1.0$ & 7.3 & 9.3 & 1.0 & na & 222 & 4.9 & na & 16.0 \\
\hline Brumley, Colo. & $3 / 28 / 1997$ & E & -8.5 & 3.9 & 5.21 & 6.2 & 3.7 & $<1.7$ & $<1.0$ & 0.6 & 1.4 & $<1.0$ & 4.0 & 7.1 & 0.8 & na & 88 & 4.9 & na & 3.2 \\
\hline $\begin{array}{l}\text { Buffalo Pass, } \\
\text { Colo. }\end{array}$ & 4/8/1997 & E & 1.2 & 2.3 & 5.86 & 1.4 & 4.7 & 1.9 & $<1.0$ & $<0.4$ & 2.6 & $<1.0$ & 9.7 & 7.1 & 0.8 & na & 432 & 6.6 & na & -26.2 \\
\hline $\begin{array}{l}\text { Cameron Pass, } \\
\text { Colo. }\end{array}$ & $3 / 26 / 1997$ & E & -13.1 & 5.7 & 4.89 & 12.9 & 3.7 & $<1.7$ & 1.4 & $<0.4$ & 2.3 & 1.4 & 6.0 & 9.3 & 0.6 & na & 190 & 6.8 & na & 9.6 \\
\hline Canyon, Wyo. & 3/13/1997 & E & -5.3 & 2.3 & 5.42 & 3.8 & $<3.1$ & $<1.7$ & $<1.0$ & $<0.4$ & 2.0 & $<1.0$ & 2.3 & 3.8 & 0.6 & na & 173 & 2.0 & na & -2.3 \\
\hline $\begin{array}{l}\text { Cement Gulch } \\
\text { Divide, Mont. }\end{array}$ & $3 / 12 / 1997$ & E & -4.1 & 4.3 & 4.91 & 12.3 & 8.9 & 2.4 & 1.2 & 4.0 & 4.9 & 1.4 & 6.9 & 7.9 & 2.7 & na & 78 & 6.6 & na & 35.2 \\
\hline $\begin{array}{l}\text { Chief Joseph } \\
\text { Pass, Mont. }\end{array}$ & $3 / 18 / 1997$ & E & -4.4 & 10.3 & 5.29 & 5.1 & $<3.1$ & $<1.7$ & $<1.0$ & $<0.4$ & 1.2 & $<1.0$ & 1.5 & 2.9 & 0.5 & na & 266 & 4.8 & na & 19.0 \\
\hline $\begin{array}{c}\text { Daisy Pass, } \\
\text { Mont. }\end{array}$ & 3/12/1997 & E & -3.9 & 2.3 & 5.41 & 3.9 & $<3.1$ & $<1.7$ & $<1.0$ & 2.1 & 1.6 & $<1.0$ & 2.3 & 3.1 & 0.6 & na & 370 & 2.9 & na & 16.9 \\
\hline $\begin{array}{l}\text { Deadman Pass, } \\
\text { Colo. }\end{array}$ & $3 / 25 / 1997$ & E & -4.8 & 4.8 & 5.29 & 5.1 & 10.3 & 2.2 & 1.2 & 2.1 & 3.4 & 1.1 & 8.3 & 11.4 & 1.3 & na & 200 & 6.9 & na & 7.8 \\
\hline $\begin{array}{l}\text { Divide Peak, } \\
\text { Wyo. }\end{array}$ & $3 / 23 / 1997$ & E & -12.2 & 3.6 & 4.95 & 11.2 & 6.1 & $<1.7$ & 1.2 & $<0.4$ & 4.3 & 1.7 & 8.5 & 13.6 & 0.8 & na & 149 & 6.2 & na & -2.0 \\
\hline Dry Lake, Colo. & $3 / 29 / 1997$ & E & -16.1 & 8.4 & 4.83 & 14.8 & 6.1 & $<1.7$ & 1.1 & $<0.4$ & 3.1 & 1.1 & 9.4 & 12.9 & 1.8 & na & 200 & 7.0 & na & 3.6 \\
\hline $\begin{array}{c}\text { Dunckley Pass, } \\
\text { Colo. }\end{array}$ & 4/2/1997 & E & -4.0 & 4.3 & 5.40 & 4.0 & 11.2 & 2.1 & 1.5 & 2.6 & 3.3 & 1.7 & 8.3 & 9.3 & 1.6 & na & 158 & 5.6 & na & 12.2 \\
\hline
\end{tabular}




\section{Table 6. Selected chemical and physical data for Rocky Mountain snowpack samples for 1997._-Continued}

[E, depth-integrated environmental; VWM, volume-weighted mean of two snowpack layers; ANC, acid neutralization capacity; $\mu$ eq/L, microequivalents per liter; $\mu \mathrm{S} / \mathrm{cm}$, microsiemens per centimeter; SC, specific conductance at $25^{\circ} \mathrm{C} ; \mathrm{pH}$ values in standard units; $\mathrm{H}$, hydrogen; $\mathrm{Ca}$, calcium; $\mathrm{Mg}$, magnesium; $\mathrm{Na}$, sodium; $\mathrm{K}$, potassium; $\mathrm{NH}_{4}$, ammonium; $\mathrm{Cl}$, chloride; $\mathrm{SO}$, sulfate; $\mathrm{NO}{ }_{3}$, nitrate; $\mathrm{DOC}$, dissolved organic carbon; mg/L, milligrams per liter; SWE, snow/water equivalent; cm, centimeters; $\delta^{34} \mathrm{~S}$, stable sulfur isotope ratio $\left.{ }^{34} \mathrm{~S} /{ }^{32} \mathrm{~S}\right) ; \mathrm{Hg}$, mercury; ng/L, nanograms per liter; na, not analyzed; <, below reporting limits; (), suspect ion balance; \%, percent]

\begin{tabular}{|c|c|c|c|c|c|c|c|c|c|c|c|c|c|c|c|c|c|c|c|c|}
\hline Site name & $\begin{array}{l}\text { Sample } \\
\text { date }\end{array}$ & $\begin{array}{l}\text { Sample } \\
\text { type }\end{array}$ & $\begin{array}{c}\text { ANC } \\
\text { ( } \mu \text { eq/L) }\end{array}$ & $\begin{array}{c}\mathrm{SC} \\
(\mu \mathrm{S} / \mathrm{cm})\end{array}$ & $\mathrm{pH}$ & $\begin{array}{c}H \\
(\mu e q / L)\end{array}$ & 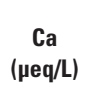 & $\begin{array}{c}\mathrm{Mg} \\
(\mu \mathrm{eq} / \mathrm{L})\end{array}$ & $\underset{(\mu \mathrm{eq} / \mathrm{L})}{\mathrm{Na}}$ & $\begin{array}{c}K \\
(\mu e q / L)\end{array}$ & $\begin{array}{c}\mathrm{NH}_{4} \\
(\mu \mathrm{eq} / \mathrm{L})\end{array}$ & $\underset{(\mu \mathrm{eq} / \mathrm{L})}{\mathrm{Cl}}$ & 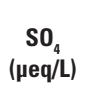 & $\begin{array}{c}\mathrm{NO}_{3} \\
\text { ( } \mu \mathrm{eq} / \mathrm{L} \text { ) }\end{array}$ & $\begin{array}{c}\text { DOC } \\
(\mu \mathrm{g} / \mathrm{L})\end{array}$ & $\begin{array}{l}\text { SWE } \\
\text { (cm) }\end{array}$ & $\begin{array}{l}\text { Snow } \\
\text { depth } \\
\text { (cm) }\end{array}$ & $\begin{array}{l}\delta^{34} \mathrm{~S} \\
\text { (per } \\
\text { mil) }\end{array}$ & $\underset{\text { (ng/L) }}{\mathrm{Hg}}$ & $\begin{array}{c}\text { lonic } \\
\text { balance } \\
(\%)\end{array}$ \\
\hline Elk River, Colo. & $3 / 29 / 1997$ & $\mathrm{E}$ & -11.6 & 6.5 & 4.96 & 11.0 & 7.2 & $<1.7$ & 1.3 & 0.9 & 2.5 & 1.4 & 7.5 & 10.7 & 1.2 & na & 144 & 6.1 & na & 7.4 \\
\hline $\begin{array}{l}\text { Elkhart Park, } \\
\text { Wyo. }\end{array}$ & $3 / 20 / 1997$ & E & -7.9 & 3.9 & 5.17 & 6.8 & $<3.1$ & $<1.7$ & $<1.0$ & 0.4 & 2.1 & 1.1 & 4.0 & 5.4 & 0.8 & na & 157 & 7.3 & na & -6.0 \\
\hline $\begin{array}{l}\text { Four Mile } \\
\text { Meadow, } \\
\text { Wyo. }\end{array}$ & $3 / 23 / 1997$ & E & -7.6 & 3.3 & 5.19 & 6.5 & 3.2 & 2.1 & $<1.0$ & 2.6 & 2.2 & 1.4 & 2.1 & 3.9 & 2.6 & na & 100 & na & na & 38.6 \\
\hline $\begin{array}{l}\text { Fremont Pass, } \\
\text { Colo. }\end{array}$ & $4 / 17 / 1997$ & $\mathrm{E}$ & -4.3 & 3.6 & 5.42 & 3.8 & 6.4 & $<1.7$ & 5.2 & 0.4 & 1.5 & 1.7 & 4.0 & 9.3 & 1.1 & na & 179 & 4.5 & na & 7.3 \\
\hline $\begin{array}{l}\text { Gallegos Peak, } \\
\text { N. Mex. }\end{array}$ & $3 / 24 / 1997$ & E & -6.1 & 4.7 & 5.26 & 5.5 & 8.1 & $<1.7$ & 1.1 & 1.4 & 4.4 & 1.1 & 9.4 & 9.3 & 0.9 & na & 134 & 3.3 & na & 1.9 \\
\hline $\begin{array}{l}\text { Garnet Canyon, } \\
\text { Wyo. }\end{array}$ & 4/20/1997 & E & -4.7 & 2.9 & 5.26 & 5.5 & $<3.1$ & $<1.7$ & $<1.0$ & 0.6 & 3.1 & 1.1 & 3.1 & 3.2 & na & na & 240 & 5.1 & na & 10.2 \\
\hline $\begin{array}{l}\text { Grand Mesa, } \\
\text { Colo. }\end{array}$ & 3/29/1997 & $\mathrm{E}$ & -9.7 & 5.3 & 5.12 & 7.6 & 7.6 & $<1.7$ & $<1.0$ & 0.4 & 3.1 & 1.1 & 8.7 & 8.6 & 1.3 & na & 205 & 4.4 & na & 0.8 \\
\hline $\begin{array}{l}\text { Granite Pass, } \\
\text { Mont. }\end{array}$ & $3 / 17 / 1997$ & E & -5.7 & 2.2 & 5.31 & 4.9 & $<3.1$ & $<1.7$ & $<1.0$ & 0.4 & $<1.0$ & $<1.0$ & 1.7 & 2.0 & 0.8 & na & 351 & 5.9 & na & 18.4 \\
\hline $\begin{array}{l}\text { Grassy Mountain, } \\
\text { Mont. }\end{array}$ & 3/11/1997 & E & 0.8 & 4.1 & 5.12 & 7.6 & 14.7 & 3.0 & 1.2 & 8.9 & 4.6 & 1.1 & 5.4 & 6.5 & 4.1 & na & 102 & na & na & $(48.5)$ \\
\hline $\begin{array}{l}\text { Gypsum Creek, } \\
\text { Wyo. }\end{array}$ & 3/20/1997 & E & -6.5 & 3.5 & 5.17 & 6.8 & $<3.1$ & $<1.7$ & $<1.0$ & $<0.4$ & 2.1 & $<1.0$ & 2.7 & 5.9 & 0.7 & na & 129 & 6.2 & na & 1.6 \\
\hline $\begin{array}{l}\text { Hogan Peak, } \\
\text { Colo. }\end{array}$ & $4 / 5 / 1997$ & E & -13.1 & 6.5 & 4.95 & 11.2 & 5.5 & $<1.7$ & 1.1 & 0.4 & 4.0 & $<1.0$ & 10.4 & 11.4 & 0.7 & na & 364 & na & na & 0.9 \\
\hline $\begin{array}{l}\text { Hopewell, } \\
\text { N. Mex. }\end{array}$ & $3 / 25 / 1997$ & E & -10.2 & 5.8 & 5.06 & 8.7 & 9.4 & $<1.7$ & 1.0 & 1.1 & 3.5 & 1.1 & 8.5 & 11.4 & 0.7 & na & 147 & 3.9 & na & 5.8 \\
\hline Kings Hill, Mont. & $3 / 14 / 1997$ & E & -6.6 & 3.6 & 5.25 & 5.6 & 3.7 & $<1.7$ & $<1.0$ & 0.4 & 3.7 & $<1.0$ & 5.2 & 5.6 & 0.7 & na & 215 & 6.2 & na & 11.1 \\
\hline Lake Irene, Colo. & 4/3/1997 & E & -6.7 & 4.7 & 5.43 & 3.7 & 3.2 & $<1.7$ & $<1.0$ & $<0.4$ & 2.9 & $<1.0$ & 5.0 & 8.6 & na & na & 149 & 5.3 & na & -15.7 \\
\hline $\begin{array}{l}\text { Lewis Lake } \\
\text { Divide, Wyo. }\end{array}$ & 3/7/1997 & E & -5.7 & 2.5 & 5.34 & 4.6 & $<3.1$ & $<1.7$ & $<1.0$ & $<0.4$ & 2.5 & $<1.0$ & 2.7 & 3.8 & 0.6 & na & 403 & 6.6 & na & 4.4 \\
\hline Lionshead, Mont. & 3/9/1997 & E & -3.8 & 3.6 & 5.38 & 4.2 & 3.2 & $<1.7$ & $<1.0$ & 1.4 & 7.1 & 1.7 & 5.0 & 7.9 & 1.2 & na & 230 & 6.0 & na & 4.5 \\
\hline $\begin{array}{l}\text { Little Brush } \\
\quad \text { Creek, Utah }\end{array}$ & $3 / 27 / 1997$ & E & -7.4 & 4.3 & 5.31 & 4.9 & 6.7 & 1.8 & 2.8 & 0.4 & 2.4 & 3.7 & 5.2 & 8.6 & na & na & 109 & 6.2 & na & 4.3 \\
\hline $\begin{array}{l}\text { Loch Vale Forest, } \\
\text { Colo. }\end{array}$ & 4/15/1997 & E & -11.6 & 5.7 & 5.03 & 9.3 & 5.6 & $<1.7$ & $<1.0$ & 0.4 & 1.9 & $<1.0$ & 7.3 & 10.0 & 0.8 & 119 & 319 & 6.0 & na & 0.1 \\
\hline $\begin{array}{l}\text { Loveland Pass, } \\
\text { Colo. }\end{array}$ & 4/18/1997 & E & -5.9 & 3.8 & 5.35 & 4.5 & 6.4 & $<1.7$ & 2.8 & $<0.4$ & 2.4 & 2.0 & 5.4 & 7.9 & na & na & 260 & na & na & 2.4 \\
\hline
\end{tabular}


[E, depth-integrated environmental; VWM, volume-weighted mean of two snowpack layers; ANC, acid neutralization capacity; $\mu$ eq/L, microequivalents per liter; $\mu \mathrm{S} / \mathrm{cm}$, microsiemens per centimeter; SC, specific conductance at $25^{\circ} \mathrm{C} ; \mathrm{pH}$ values in standard units; $\mathrm{H}$, hydrogen; $\mathrm{Ca}$, calcium; $\mathrm{Mg}$, magnesium; $\mathrm{Na}$, sodium; $\mathrm{K}$, potassium; $\mathrm{NH}_{4}$, ammonium; $\mathrm{Cl}$, chloride; $\mathrm{SO}$, sulfate; $\mathrm{NO}{ }_{3}$, nitrate; $\mathrm{DOC}$, dissolved organic carbon; $\mathrm{mg} / \mathrm{L}$, milligrams per liter; SWE, snow/water equivalent; cm, centimeters; $\delta^{34} \mathrm{~S}$, stable sulfur isotope ratio $\left.{ }^{34} \mathrm{~S} /{ }^{32} \mathrm{~S}\right) ; \mathrm{Hg}$, mercury; ng/L, nanograms per liter; na, not analyzed; <, below reporting limits; (), suspect ion balance; \%, percent]

\begin{tabular}{|c|c|c|c|c|c|c|c|c|c|c|c|c|c|c|c|c|c|c|c|c|}
\hline Site name & $\begin{array}{l}\text { Sample } \\
\text { date }\end{array}$ & $\begin{array}{l}\text { Sample } \\
\text { type }\end{array}$ & $\begin{array}{c}\text { ANC } \\
\text { ( } \mu \mathrm{eq} / \mathrm{L})\end{array}$ & $\begin{array}{c}\mathrm{SC} \\
(\mu \mathrm{S} / \mathrm{cm})\end{array}$ & pH & 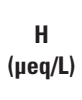 & 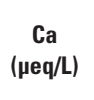 & $\underset{(\mu \mathrm{eq} / \mathrm{L})}{\mathrm{Mg}}$ & $\begin{array}{c}\mathrm{Na} \\
(\mu \mathrm{eq} / \mathrm{L})\end{array}$ & $\begin{array}{c}K \\
(\mu e q / L)\end{array}$ & $\underset{(\mu \mathrm{eq} / \mathrm{L})}{\mathrm{NH}_{4}}$ & $\begin{array}{c}C I \\
(\mu e q / L)\end{array}$ & $\begin{array}{c}\mathrm{SO}_{4} \\
(\mu \mathrm{eq} / \mathrm{L})\end{array}$ & $\begin{array}{c}\mathrm{NO}_{3} \\
(\mu \mathrm{eq} / \mathrm{L})\end{array}$ & $\begin{array}{l}\text { DOC } \\
(\mu \mathrm{g} / \mathrm{L})\end{array}$ & $\begin{array}{l}\text { SWE } \\
\text { (cm) }\end{array}$ & $\begin{array}{l}\text { Snow } \\
\text { depth } \\
\text { (cm) }\end{array}$ & $\begin{array}{l}\delta^{34} S \\
\text { (per } \\
\text { mil) }\end{array}$ & $\begin{array}{c}\mathrm{Hg} \\
\text { (ng/L) }\end{array}$ & $\begin{array}{c}\text { lonic } \\
\text { balance } \\
(\%)\end{array}$ \\
\hline Lynx Pass, Colo. & $3 / 28 / 1997$ & E & -11.7 & 5.0 & 5.04 & 9.1 & 4.8 & $<1.7$ & $<1.0$ & 0.9 & 1.7 & $<1.0$ & 5.8 & 8.6 & 1.0 & na & 136 & 5.1 & na & 6.8 \\
\hline $\begin{array}{l}\text { Molas Lake, } \\
\text { Colo. }\end{array}$ & 4/12/1997 & E & -3.6 & 3.8 & 5.54 & 2.9 & 12.3 & $<1.7$ & 1.4 & 0.4 & 2.2 & $<1.0$ & 5.6 & 9.3 & 0.9 & na & 194 & 4.6 & na & 12.6 \\
\hline $\begin{array}{l}\text { Monarch Pass, } \\
\text { Colo. }\end{array}$ & $3 / 28 / 1997$ & E & -3.1 & 2.7 & 5.55 & 2.8 & 6.6 & $<1.7$ & $<1.0$ & $<0.4$ & 1.6 & $<1.0$ & 4.0 & 5.9 & na & na & 128 & na & na & 5.2 \\
\hline $\begin{array}{l}\text { Monida Pass, } \\
\text { Mont. }\end{array}$ & $3 / 10 / 1997$ & E & -2.0 & 4.5 & 5.03 & 9.3 & 8.3 & 2.7 & 1.5 & 5.5 & 5.9 & 2.5 & 3.7 & 7.9 & na & na & 65 & na & na & 40.5 \\
\hline $\begin{array}{l}\text { Mount Belmont, } \\
\text { Mont. }\end{array}$ & 3/13/1997 & E & -8.1 & 5.1 & 4.94 & 11.5 & 3.7 & $<1.7$ & $<1.0$ & 1.4 & 3.3 & $<1.0$ & 5.2 & 4.8 & 1.6 & na & 137 & na & na & 33.2 \\
\hline $\begin{array}{l}\text { Niwot Snotel, } \\
\text { Colo. }\end{array}$ & 4/8/1997 & E & -8.4 & 4.4 & 5.18 & 6.6 & 3.8 & $<1.7$ & $<1.0$ & 0.4 & 5.2 & $<1.0$ & 6.2 & 7.9 & na & na & 145 & 5.3 & na & 6.5 \\
\hline $\begin{array}{l}\text { Noisy Basin, } \\
\text { Mont. }\end{array}$ & $3 / 15 / 1997$ & E & -7.6 & 3.6 & 5.15 & 7.1 & $<3.1$ & $<1.7$ & $<1.0$ & 0.4 & 2.1 & 1.1 & 3.7 & 4.7 & na & na & 496 & 6.8 & na & -0.1 \\
\hline Old Battle, Wyo. & 3/22/1997 & E & -12.2 & 6.5 & 4.95 & 11.2 & 5.2 & $<1.7$ & 1.0 & $<0.4$ & 3.0 & 1.1 & 8.3 & 10.7 & 0.7 & na & 270 & 6.6 & na & 0.8 \\
\hline $\begin{array}{l}\text { Old Faithful } \\
\text { Crew Corrals, } \\
\text { Wyo. }\end{array}$ & 3/11/1997 & E & -5.7 & 2.1 & 5.40 & 4.0 & $<3.1$ & $<1.7$ & $<1.0$ & $<0.4$ & 2.0 & $<1.0$ & 1.2 & 3.3 & 0.5 & na & 160 & na & na & 13.9 \\
\hline $\begin{array}{l}\text { Old Faithful East } \\
\text { Lot, Wyo. }\end{array}$ & $3 / 11 / 1997$ & E & -5.8 & 2.6 & 5.33 & 4.7 & $<3.1$ & $<1.7$ & 1.3 & $<0.4$ & 2.2 & 1.1 & 1.7 & 3.5 & 0.6 & na & 162 & na & na & 13.1 \\
\hline $\begin{array}{l}\text { Old Faithful Fire } \\
\text { Road, Wyo. }\end{array}$ & $3 / 11 / 1997$ & E & -4.8 & 2.7 & 5.34 & 4.6 & $<3.1$ & $<1.7$ & $<1.0$ & 0.4 & 1.9 & $<1.0$ & 2.1 & 4.1 & 1.0 & na & 190 & na & na & 5.3 \\
\hline $\begin{array}{l}\text { Phantom Valley, } \\
\text { Colo. }\end{array}$ & 4/2/1997 & E & -9.2 & 4.5 & 5.12 & 7.6 & 4.1 & $<1.7$ & 3.2 & 1.4 & 3.0 & 2.0 & 5.2 & 9.3 & na & na & 84 & 5.5 & na & 8.0 \\
\hline $\begin{array}{l}\text { Rabbit Ears 1, } \\
\text { Colo. }\end{array}$ & 4/1/1997 & E & -14.5 & 6.6 & 4.95 & 11.2 & 4.8 & $<1.7$ & $<1.0$ & 0.4 & 3.1 & $<1.0$ & 8.9 & 9.3 & 0.7 & na & 260 & 6.7 & na & 3.5 \\
\hline $\begin{array}{l}\text { Rabbit Ears 2, } \\
\quad \text { Colo. }\end{array}$ & 4/1/1997 & E & -15.0 & 6.9 & 4.87 & 13.5 & $<3.1$ & $<1.7$ & $<1.0$ & $<0.4$ & 2.9 & $<1.0$ & 9.6 & 7.1 & 0.7 & na & 250 & 7.0 & na & -1.1 \\
\hline $\begin{array}{l}\text { Red Mountain, } \\
\text { Mont. }\end{array}$ & $3 / 19 / 1997$ & E & -3.7 & 3.1 & 5.32 & 4.8 & 3.7 & $<1.7$ & $<1.0$ & 0.4 & 4.6 & $<1.0$ & 3.5 & 6.5 & 1.1 & na & 198 & 6.2 & na & 14.7 \\
\hline $\begin{array}{l}\text { Red Mountain } \\
\text { Pass, Colo. }\end{array}$ & 4/12/1997 & E & 7.4 & 4.6 & 6.13 & 0.7 & 24.2 & 3.9 & 2.0 & 2.6 & 2.4 & 1.4 & 7.1 & 10.0 & 0.8 & na & 362 & 3.9 & na & 16.2 \\
\hline $\begin{array}{l}\text { Rendezvous } \\
\text { Mountain, } \\
\text { Wyo. }\end{array}$ & 3/21/1997 & E & -15.6 & 7.8 & 4.90 & 12.6 & 5.2 & $<1.7$ & 1.1 & $<0.4$ & 3.6 & $<1.0$ & 3.1 & 9.3 & 0.8 & na & 366 & 5.7 & na & 28.8 \\
\hline $\begin{array}{l}\text { Ripple Creek } \\
\text { Pass, Colo. }\end{array}$ & 4/2/1997 & E & -2.9 & 3.4 & 5.43 & 3.7 & 6.6 & $<1.7$ & 1.3 & 0.4 & 2.2 & $<1.0$ & 4.6 & 6.6 & 0.7 & na & 218 & 5.5 & na & 12.2 \\
\hline
\end{tabular}




\section{Table 6. Selected chemical and physical data for Rocky Mountain snowpack samples for 1997.—Continued}

[E, depth-integrated environmental; VWM, volume-weighted mean of two snowpack layers; ANC, acid neutralization capacity; $\mu$ eq/L, microequivalents per liter; $\mu \mathrm{S} / \mathrm{cm}$, microsiemens per centimeter; SC, specific conductance at $25^{\circ} \mathrm{C} ; \mathrm{pH}$ values in standard units; $\mathrm{H}$, hydrogen; $\mathrm{Ca}$, calcium; $\mathrm{Mg}$, magnesium; $\mathrm{Na}$, sodium; $\mathrm{K}$, potassium; $\mathrm{NH}_{4}$, ammonium; $\mathrm{Cl}$, chloride; $\mathrm{SO}$, sulfate; $\mathrm{NO}{ }_{3}$, nitrate; $\mathrm{DOC}$, dissolved organic carbon; $\mathrm{mg} / \mathrm{L}$, milligrams per liter; SWE, snow/water equivalent; cm, centimeters; $\delta^{34} \mathrm{~S}$, stable sulfur isotope ratio $\left.{ }^{34} \mathrm{~S} /{ }^{32} \mathrm{~S}\right) ; \mathrm{Hg}$, mercury; ng/L, nanograms per liter; na, not analyzed; <, below reporting limits; (), suspect ion balance; \%, percent]

\begin{tabular}{|c|c|c|c|c|c|c|c|c|c|c|c|c|c|c|c|c|c|c|c|c|}
\hline Site name & $\begin{array}{l}\text { Sample } \\
\text { date }\end{array}$ & $\begin{array}{l}\text { Sample } \\
\text { type }\end{array}$ & $\begin{array}{c}\text { ANC } \\
(\mu \mathrm{eq} / \mathrm{L})\end{array}$ & $\begin{array}{c}\text { SC } \\
(\mu \mathrm{S} / \mathrm{cm})\end{array}$ & pH & $\begin{array}{c}H \\
(\mu \mathrm{eq} / \mathrm{L})\end{array}$ & $\begin{array}{c}\text { Ca } \\
(\mu \mathrm{eq} / \mathrm{L})\end{array}$ & $\begin{array}{c}\mathrm{Mg} \\
(\mu \mathrm{eq} / \mathrm{L})\end{array}$ & $\begin{array}{c}\mathrm{Na} \\
(\mu \mathrm{eq} / \mathrm{L})\end{array}$ & $\begin{array}{c}K \\
(\mu e q / L)\end{array}$ & $\begin{array}{c}\mathrm{NH}_{4} \\
(\mu \mathrm{eq} / \mathrm{L})\end{array}$ & $\begin{array}{c}C I \\
(\mu \mathrm{eq} / \mathrm{L})\end{array}$ & $\begin{array}{c}\mathrm{SO}_{4} \\
(\mu \mathrm{eq} / \mathrm{L})\end{array}$ & $\begin{array}{c}\mathrm{NO}_{3} \\
(\mu \mathrm{eq} / \mathrm{L})\end{array}$ & $\begin{array}{c}\text { DOC } \\
(\mu \mathrm{g} / \mathrm{L})\end{array}$ & $\begin{array}{l}\text { SWE } \\
\text { (cm) }\end{array}$ & $\begin{array}{l}\text { Snow } \\
\text { depth } \\
\text { (cm) }\end{array}$ & $\begin{array}{l}\delta^{34} S \\
\text { (per } \\
\text { mil) } \\
\end{array}$ & $\begin{array}{c}\mathrm{Hg} \\
(\mathbf{n g} / \mathrm{L})\end{array}$ & $\begin{array}{c}\text { Ionic } \\
\text { balance } \\
(\%) \\
\end{array}$ \\
\hline $\begin{array}{l}\text { Slumgullion Pass, } \\
\text { Colo. }\end{array}$ & 4/11/1997 & E & 0.5 & 3.6 & 5.72 & 1.9 & 12.9 & 1.7 & 1.4 & 1.1 & 2.4 & 1.1 & 5.6 & 6.6 & 0.9 & na & 198 & 4.1 & na & 21.6 \\
\hline $\begin{array}{l}\text { Snow Bowl, } \\
\text { Mont. }\end{array}$ & $3 / 17 / 1997$ & E & -7.6 & 3.6 & 5.11 & 7.8 & $<3.1$ & $<1.7$ & $<1.0$ & $<0.4$ & 1.6 & $<1.0$ & 2.7 & 4.0 & 0.7 & na & 348 & 7.4 & na & 16.4 \\
\hline South Pass, Wyo. & 3/23/1997 & E & -7.5 & 5.8 & 5.13 & 7.4 & 8.0 & 1.9 & 3.3 & 0.6 & 2.6 & 1.4 & 9.2 & 8.6 & 1.3 & na & 166 & 7.4 & na & 11.0 \\
\hline $\begin{array}{l}\text { Spring Gulch, } \\
\text { Mont. }\end{array}$ & $3 / 12 / 1997$ & E & -4.2 & 7.0 & 5.10 & 7.9 & 5.4 & $<1.7$ & 1.2 & 1.1 & 4.1 & 2.0 & 5.2 & 7.1 & na & na & 93 & 6.3 & na & 16.0 \\
\hline $\begin{array}{l}\text { Sunlight Creek, } \\
\text { Mont. }\end{array}$ & $3 / 11 / 1997$ & E & -5.4 & 3.5 & 5.20 & 6.3 & $<3.1$ & $<1.7$ & $<1.0$ & $<0.4$ & 3.6 & $<1.0$ & 4.2 & 6.0 & 0.7 & na & 172 & na & na & -1.4 \\
\hline $\begin{array}{l}\text { Sunlight Peak, } \\
\text { Colo. }\end{array}$ & 4/1/1997 & E & -7.5 & 4.0 & 5.27 & 5.4 & 6.1 & $<1.7$ & $<1.0$ & 0.4 & 2.8 & $<1.0$ & 6.0 & 8.6 & 0.7 & na & 205 & 5.2 & na & 0.2 \\
\hline $\begin{array}{l}\text { Sylvan Lake, } \\
\text { Wyo. }\end{array}$ & 3/8/1997 & E & -4.2 & 2.4 & 5.33 & 4.7 & $<3.1$ & $<1.7$ & $<1.0$ & $<0.4$ & 2.2 & $<1.0$ & 2.3 & 3.6 & 0.7 & na & 272 & 3.8 & na & 8.1 \\
\hline Teton Pass, Wyo. & $3 / 21 / 1997$ & $\mathrm{E}$ & -4.6 & 3.4 & 5.31 & 4.9 & 3.1 & $<1.7$ & $<1.0$ & 1.1 & 3.5 & $<1.0$ & 3.7 & 4.0 & 1.3 & na & 278 & 5.2 & na & 24.0 \\
\hline $\begin{array}{l}\text { Togwotee Pass, } \\
\text { Wyo. }\end{array}$ & $3 / 23 / 1997$ & E & -10.6 & 3.5 & 5.16 & 6.9 & $<3.1$ & $<1.7$ & $<1.0$ & $<0.4$ & 1.2 & $<1.0$ & 2.5 & 1.7 & 0.8 & na & 258 & 5.7 & na & 31.8 \\
\hline $\begin{array}{l}\text { Trappers Lake, } \\
\text { Colo. }\end{array}$ & 4/2/1997 & E & -1.3 & 4.0 & 5.47 & 3.4 & 8.5 & 1.7 & 1.8 & 1.6 & 2.7 & 1.7 & 6.7 & 8.6 & 1.7 & na & 200 & 5.9 & na & 7.5 \\
\hline Trial Lake, Utah & $3 / 26 / 1997$ & E & -4.3 & 3.2 & 5.31 & 4.9 & 5.5 & $<1.7$ & 1.5 & 0.6 & 2.0 & 1.7 & 3.7 & 6.5 & 1.1 & na & 231 & 5.6 & na & 9.6 \\
\hline $\begin{array}{l}\text { Twenty-one Mile, } \\
\text { Mont. }\end{array}$ & 3/10/1997 & E & -6.3 & 3.1 & 5.30 & 5.0 & $<3.1$ & $<1.7$ & $<1.0$ & $<0.4$ & 3.0 & 1.1 & 2.7 & 5.4 & 0.6 & na & 213 & 4.6 & na & -7.2 \\
\hline $\begin{array}{l}\text { University Camp, } \\
\text { Colo. }\end{array}$ & 4/9/1997 & E & -10.4 & 5.3 & 5.10 & 7.9 & 5.1 & $<1.7$ & $<1.0$ & 0.4 & 3.9 & $<1.0$ & 6.9 & 9.3 & 1.1 & na & 198 & 6.0 & na & 3.4 \\
\hline $\begin{array}{l}\text { West Yellow- } \\
\text { stone, Mont. }\end{array}$ & 3/10/1997 & E & -5.7 & 4.6 & 5.23 & 5.9 & $<3.1$ & $<1.7$ & $<1.0$ & 0.4 & 3.6 & $<1.0$ & 2.7 & 4.1 & 0.9 & na & 152 & 5.0 & na & 18.6 \\
\hline $\begin{array}{l}\text { Willow Creek, } \\
\text { Wyo. }\end{array}$ & $3 / 24 / 1997$ & E & -7.1 & 3.7 & 5.25 & 5.6 & 3.2 & $<1.7$ & 1.1 & 0.6 & 3.7 & 1.7 & 4.4 & 5.5 & 0.8 & na & 260 & 6.0 & na & 10.4 \\
\hline $\begin{array}{l}\text { Wolf Creek Pass, } \\
\text { Colo. }\end{array}$ & 4/13/1997 & E & 5.8 & 4.4 & 5.98 & 1.0 & 18.4 & 1.9 & 2.9 & 0.6 & 3.5 & 1.7 & 8.1 & 8.6 & 0.9 & na & 290 & 3.9 & na & 7.9 \\
\hline
\end{tabular}


[E, depth-integrated environmental; ANC, acid neutralization capacity; $\mu \mathrm{eq} / \mathrm{L}$, microequivalents per liter; $\mu \mathrm{S} / \mathrm{cm}$, microsiemens per centimeter; $\mathrm{SC}$, specific conductance at $25^{\circ} \mathrm{C} ; \mathrm{pH}$ values in standard units; $\mathrm{H}$, hydrogen; $\mathrm{Ca}$, calcium; $\mathrm{Mg}$, magnesium; $\mathrm{Na}$, sodium; $\mathrm{K}$, potassium; $\mathrm{NH}_{4}$, ammonium; $\mathrm{Cl}$, chloride; $\mathrm{SO}_{4}$, sulfate; $\mathrm{NO}_{3}$, nitrate; DOC, dissolved organic carbon; mg/L, milligrams per liter; $\mathrm{SWE}$, snow/water equivalent; cm, centimeters; $\delta^{34} \mathrm{~S}$, stable sulfur isotope ratio $\left({ }^{34} \mathrm{~S} /{ }^{22} \mathrm{~S}\right) ; \mathrm{Hg}$, mercury; ng/L, nanograms per liter; na, not analyzed; <, below reporting limits; NP, National Park; \%, percent]

\begin{tabular}{|c|c|c|c|c|c|c|c|c|c|c|c|c|c|c|c|c|c|c|c|c|}
\hline Site name & $\begin{array}{l}\text { Sample } \\
\text { date }\end{array}$ & $\begin{array}{l}\text { Sample } \\
\text { type }\end{array}$ & $\begin{array}{c}\text { ANC } \\
(\mu \mathrm{eq} / \mathrm{L})\end{array}$ & $\begin{array}{c}\mathrm{SC} \\
(\mu \mathrm{S} / \mathrm{cm})\end{array}$ & $\mathrm{pH}$ & $\underset{(\mu \mathrm{eq} / \mathrm{L})}{\mathrm{H}}$ & $\underset{(\mu \mathrm{eq} / \mathrm{L})}{\mathrm{Ca}}$ & $\underset{(\mu \mathrm{eq} / \mathrm{L})}{\mathrm{Mg}}$ & $\underset{(\mu \mathrm{eq} / \mathrm{L})}{\mathrm{Na}}$ & $\begin{array}{c}K \\
\text { ( } \mu \text { eq/L) }\end{array}$ & 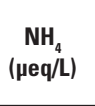 & $\underset{(\mu \mathrm{eq} / \mathrm{L})}{\mathrm{Cl}}$ & $\begin{array}{c}\mathrm{SO}_{4} \\
\text { (peq/L) }\end{array}$ & $\begin{array}{c}\mathrm{NO}_{3} \\
(\mu \mathrm{eq} / \mathrm{L})\end{array}$ & $\begin{array}{l}\text { DOC } \\
(\mu \mathrm{g} / \mathrm{L})\end{array}$ & $\begin{array}{l}\text { SWE } \\
\text { (cm) }\end{array}$ & $\begin{array}{l}\text { Snow } \\
\text { depth } \\
(\mathrm{cm})\end{array}$ & $\begin{array}{c}\delta^{34} \mathbf{S} \\
\text { (per mil) }\end{array}$ & $\underset{(\mathrm{ng} / \mathrm{L})}{\mathrm{Hg}}$ & $\begin{array}{c}\text { lonic } \\
\text { balance } \\
(\%)\end{array}$ \\
\hline $\begin{array}{l}\text { Apgar Lookout, } \\
\text { Mont. }\end{array}$ & $3 / 5 / 1998$ & E & -11.0 & 4.9 & 4.99 & 10.2 & $<3.1$ & $<1.7$ & 2.2 & $<0.4$ & 6.1 & 1.4 & 6.0 & 6.9 & 0.7 & na & 144 & 6.3 & na & 12.5 \\
\hline $\begin{array}{l}\text { Beaver Moun- } \\
\text { tain, Utah }\end{array}$ & $3 / 26 / 1998$ & E & -1.5 & 3.8 & 5.72 & 1.9 & 11.0 & $<1.7$ & 4.4 & 0.5 & 5.4 & 3.7 & 5.6 & 7.5 & 0.5 & na & 170 & 6.8 & na & 15.9 \\
\hline $\begin{array}{l}\text { Berthoud Pass, } \\
\text { Colo. }\end{array}$ & 4/15/1998 & $\mathrm{E}$ & -9.1 & 4.9 & 5.06 & 8.7 & 5.5 & $<1.7$ & 4.8 & 0.8 & 3.5 & 5.1 & 5.6 & 8.9 & 0.8 & na & 196 & 6.0 & na & 8.6 \\
\hline $\begin{array}{l}\text { Big Mountain, } \\
\text { Mont. }\end{array}$ & $3 / 13 / 1998$ & E & -7.8 & 3.1 & 5.14 & 7.2 & $<3.1$ & $<1.7$ & 1.3 & $<0.4$ & 3.1 & 1.1 & 3.5 & 5.0 & 0.5 & na & 231 & 7.5 & na & 9.0 \\
\hline Big Sky, Mont. & 3/18/1998 & E & -5.5 & 2.9 & 5.24 & 5.8 & 3.5 & $<1.7$ & $<1.0$ & $<0.4$ & 3.7 & $<1.0$ & 3.5 & 5.4 & 0.7 & na & 128 & 6.1 & na & 18.4 \\
\hline $\begin{array}{l}\text { Biscuit Basin, } \\
\text { Wyo. }\end{array}$ & $3 / 6 / 1998$ & E & -5.4 & 7.7 & 5.19 & 6.5 & 10.5 & 4.1 & 4.8 & 0.8 & 21.9 & 7.6 & 16.2 & 10.0 & 2.8 & na & 101 & na & na & 17.8 \\
\hline $\begin{array}{l}\text { Brooklyn Lake, } \\
\text { Wyo. }\end{array}$ & $3 / 25 / 1998$ & E & -12.6 & 5.9 & 4.96 & 11.0 & 6.0 & $<1.7$ & 1.3 & 1.3 & 4.4 & 1.4 & 8.1 & 10.7 & 0.7 & na & 228 & 7.3 & na & 8.5 \\
\hline Brumley, Colo. & $3 / 17 / 1998$ & E & -11.9 & 5.0 & 4.93 & 11.7 & $<3.1$ & $<1.7$ & 1.3 & $<0.4$ & 2.7 & $<1.0$ & 5.4 & 8.4 & $<0.4$ & na & 85 & 4.8 & na & 6.6 \\
\hline $\begin{array}{l}\text { Buffalo Pass, } \\
\text { Colo. }\end{array}$ & 4/1/1998 & E & -22.1 & 8.3 & 4.72 & 19.1 & 3.5 & $<1.7$ & $<1.0$ & $<0.4$ & 4.7 & $<1.0$ & 11.0 & 11.0 & 0.7 & na & 342 & 7.8 & na & 10.5 \\
\hline $\begin{array}{l}\text { Cameron Pass, } \\
\text { Colo. }\end{array}$ & 4/9/1998 & E & -12.4 & 6.4 & 4.91 & 12.3 & 5.5 & $<1.7$ & 2.2 & 0.5 & 4.0 & 2.0 & 8.7 & 10.7 & 0.8 & na & 165 & 6.2 & na & 6.6 \\
\hline Canyon, Wyo. & $3 / 8 / 1998$ & E & -7.0 & 3.8 & 5.11 & 7.8 & $<3.1$ & $<1.7$ & $<1.0$ & $<0.4$ & 4.2 & 1.4 & 3.3 & 6.8 & 0.5 & na & 110 & 4.7 & na & 1.6 \\
\hline $\begin{array}{l}\text { Cement Gulch } \\
\text { Divide, } \\
\text { Mont. }\end{array}$ & $3 / 10 / 1998$ & $\mathrm{E}$ & -6.5 & 4.3 & 5.18 & 6.6 & 4.5 & $<1.7$ & $<1.0$ & $<0.4$ & 6.7 & 1.1 & 5.6 & 9.1 & 0.7 & na & 73 & 5.2 & na & 6.0 \\
\hline $\begin{array}{l}\text { Chief Joseph } \\
\text { Pass, Mont. }\end{array}$ & $3 / 15 / 1998$ & E & -6.7 & 2.9 & 5.25 & 5.6 & $<3.1$ & $<1.7$ & $<1.0$ & $<0.4$ & 3.9 & $<1.0$ & 3.1 & 4.7 & 0.6 & na & 140 & 6.8 & na & 10.1 \\
\hline $\begin{array}{l}\text { Daisy Pass, } \\
\text { Mont. }\end{array}$ & 3/9/1998 & E & -7.1 & 3.0 & 5.19 & 6.5 & $<3.1$ & $<1.7$ & $<1.0$ & 0.5 & 2.8 & $<1.0$ & 3.5 & 5.0 & 0.9 & na & 212 & 4.4 & na & 6.8 \\
\hline $\begin{array}{l}\text { Deadman Pass, } \\
\text { Colo. }\end{array}$ & 3/23/1998 & E & -11.9 & 5.7 & 4.93 & 11.7 & 5.5 & $<1.7$ & $<1.0$ & $<0.4$ & 4.2 & $<1.0$ & 7.1 & 11.1 & 0.7 & na & 193 & 6.8 & na & 8.2 \\
\hline $\begin{array}{l}\text { Divide Peak, } \\
\text { Wyo. }\end{array}$ & $3 / 24 / 1998$ & E & -22.9 & 10.5 & 4.67 & 21.4 & 7.0 & $<1.7$ & 2.2 & $<0.4$ & 5.7 & 1.7 & 12.5 & 19.5 & 0.6 & na & 222 & 7.4 & na & 3.6 \\
\hline Dry Lake, Colo. & $3 / 10 / 1998$ & $\mathrm{E}$ & -22.9 & 10.4 & 4.65 & 22.4 & 4.0 & $<1.7$ & $<1.0$ & $<0.4$ & 6.6 & $<1.0$ & 12.9 & 15.8 & 0.5 & na & 138 & 8.6 & na & 7.0 \\
\hline $\begin{array}{l}\text { Dunckley Pass, } \\
\text { Colo. }\end{array}$ & 4/1/1998 & E & -11.1 & 5.4 & 4.94 & 11.5 & 5.5 & $<1.7$ & $<1.0$ & 1.5 & 5.0 & 1.1 & 6.9 & 11.2 & 0.6 & na & 157 & 6.0 & na & 10.1 \\
\hline Elk River, Colo. & $3 / 12 / 1998$ & E & -20.5 & 8.4 & 4.70 & 20.0 & 7.5 & $<1.7$ & 2.2 & 0.5 & 4.0 & 1.1 & 11.0 & 16.6 & 0.6 & na & 136 & 5.3 & na & 8.5 \\
\hline
\end{tabular}




\section{Table 7. Selected chemical and physical data for Rocky Mountain snowpack samples for 1998.—Continued}

[E, depth-integrated environmental; $\mathrm{ANC}$, acid neutralization capacity; $\mu \mathrm{eq} / \mathrm{L}$, microequivalents per liter; $\mu \mathrm{S} / \mathrm{cm}$, microsiemens per centimeter; $\mathrm{SC}$, specific conductance at $25^{\circ} \mathrm{C}$; $\mathrm{pH}$ values in standard units; $\mathrm{H}$, hydrogen; $\mathrm{Ca}$, calcium; $\mathrm{Mg}$, magnesium; $\mathrm{Na}$, sodium; $\mathrm{K}$, potassium; $\mathrm{NH}_{4}$, ammonium; $\mathrm{Cl}$, chloride; $\mathrm{SO}_{4}$, sulfate; $\mathrm{NO}_{3}$, nitrate; $\mathrm{DOC}$, dissolved organic carbon; mg/L, milligrams per liter; $\mathrm{SWE}$, snow/water equivalent; cm, centimeters; $\delta^{34} \mathrm{~S}$, stable sulfur isotope ratio $\left({ }^{34} \mathrm{~S} /{ }^{32} \mathrm{~S}\right) ; \mathrm{Hg}$, mercury; ng/L, nanograms per liter; na, not analyzed; <, below reporting limits; NP, National Park; \%, percent]

\begin{tabular}{|c|c|c|c|c|c|c|c|c|c|c|c|c|c|c|c|c|c|c|c|c|}
\hline Site name & $\begin{array}{l}\text { Sample } \\
\text { date }\end{array}$ & $\begin{array}{c}\text { Sample } \\
\text { type }\end{array}$ & $\begin{array}{c}\text { ANC } \\
\text { ( } \mu \text { eq/L) }\end{array}$ & $\begin{array}{c}S C \\
(\mu \mathrm{S} / \mathrm{cm})\end{array}$ & $\mathrm{pH}$ & $\begin{array}{c}\text { H } \\
(\mu \mathrm{eq} / \mathrm{L})\end{array}$ & $\begin{array}{c}\mathrm{Ca} \\
(\mu \mathrm{eq} / \mathrm{L})\end{array}$ & $\begin{array}{c}\mathrm{Mg} \\
\text { (peq/L) }\end{array}$ & $\begin{array}{c}\mathrm{Na} \\
(\mu \mathrm{eq} / \mathrm{L})\end{array}$ & $\begin{array}{c}K \\
\text { (peq/L) }\end{array}$ & $\begin{array}{c}\mathrm{NH}_{4} \\
(\mu \mathrm{eq} / \mathrm{L})\end{array}$ & $\begin{array}{c}C l \\
(\mu e q / L)\end{array}$ & $\begin{array}{c}\mathrm{SO}_{4} \\
(\mu \mathrm{eq} / \mathrm{L})\end{array}$ & $\begin{array}{c}\mathrm{NO}_{3} \\
\text { ( } \mu \mathrm{eq} / \mathrm{L})\end{array}$ & $\begin{array}{c}D O C \\
(\mu \mathrm{g} / \mathrm{L})\end{array}$ & $\begin{array}{l}\text { SWE } \\
\text { (cm) }\end{array}$ & $\begin{array}{l}\text { Snow } \\
\text { depth } \\
\text { (cm) }\end{array}$ & $\begin{array}{c}\delta^{34} \mathbf{S} \\
\text { (per mil) }\end{array}$ & $\begin{array}{c}\mathrm{Hg} \\
\text { (ng/L) }\end{array}$ & $\begin{array}{c}\text { Ionic } \\
\text { balance } \\
(\%)\end{array}$ \\
\hline $\begin{array}{c}\text { Elkhart Park, } \\
\text { Wyo. }\end{array}$ & $3 / 25 / 1998$ & $\mathrm{E}$ & -7.0 & 4.5 & 5.27 & 5.4 & 6.5 & $<1.7$ & 5.7 & 0.5 & 3.7 & 2.3 & 8.1 & 7.8 & 0.4 & na & 126 & 7.3 & na & 9.0 \\
\hline $\begin{array}{l}\text { Four Mile } \\
\text { Meadow, } \\
\text { Wyo. }\end{array}$ & $3 / 24 / 1998$ & E & -5.9 & 3.2 & 5.18 & 6.6 & 3.5 & $<1.7$ & 2.2 & $<0.4$ & 2.5 & 1.4 & 3.7 & 6.2 & 0.5 & na & 89 & 7.9 & na & 13.2 \\
\hline $\begin{array}{l}\text { Fremont Pass, } \\
\text { Colo. }\end{array}$ & $4 / 16 / 1998$ & $\mathrm{E}$ & -5.8 & 4.3 & 5.21 & 6.2 & 8.0 & $<1.7$ & 2.2 & $<0.4$ & 2.7 & 2.5 & 4.4 & 9.0 & 0.4 & na & 171 & 5.1 & na & 9.1 \\
\hline $\begin{array}{l}\text { Gallegos Peak, } \\
\text { N. Mex. }\end{array}$ & $3 / 26 / 1998$ & E & -6.2 & 4.9 & 5.34 & 4.6 & 15.0 & $<1.7$ & $<1.0$ & 1.3 & 6.6 & 1.1 & 11.9 & 9.6 & 1.2 & na & 118 & 4.6 & na & 9.5 \\
\hline $\begin{array}{l}\text { Garnet Canyon, } \\
\text { Wyo. }\end{array}$ & $3 / 16 / 1998$ & E & -9.7 & 3.4 & 5.08 & 8.3 & $<3.1$ & $<1.7$ & $<1.0$ & $<0.4$ & 3.5 & 1.4 & 3.7 & 5.7 & 0.5 & na & 178 & 6.3 & na & 4.3 \\
\hline $\begin{array}{l}\text { Grand Mesa, } \\
\text { Colo. }\end{array}$ & $3 / 30 / 1998$ & $\mathrm{E}$ & -9.1 & 5.3 & 5.10 & 7.9 & 8.0 & $<1.7$ & 1.3 & 0.5 & 5.1 & 1.1 & 10.2 & 9.2 & 0.7 & na & 195 & 3.5 & na & 5.2 \\
\hline $\begin{array}{l}\text { Granite Pass, } \\
\text { Mont. }\end{array}$ & 3/16/1998 & E & -6.8 & 2.7 & 5.23 & 5.9 & $<3.1$ & $<1.7$ & 3.0 & 0.8 & 1.0 & 2.0 & 2.9 & 3.5 & 0.8 & na & 150 & 7.1 & na & 12.3 \\
\hline $\begin{array}{l}\text { Grassy Moun- } \\
\text { tain, Mont. }\end{array}$ & 3/9/1998 & E & -7.1 & 5.4 & 5.14 & 7.2 & 12.0 & 2.5 & $<1.0$ & 4.6 & 6.9 & 2.8 & 7.9 & 11.9 & 1.3 & na & 63 & na & na & 19.0 \\
\hline $\begin{array}{l}\text { Hogan Peak, } \\
\text { Colo. }\end{array}$ & 4/11/1998 & E & -19.6 & 9.5 & 4.75 & 17.8 & 4.0 & $<1.7$ & $<1.0$ & 3.8 & 7.1 & 1.7 & 14.6 & 12.5 & 1.1 & na & 242 & na & na & 6.5 \\
\hline $\begin{array}{l}\text { Hopewell, } \\
\text { N. Mex. }\end{array}$ & $3 / 27 / 1998$ & E & -10.2 & 6.6 & 5.01 & 9.8 & 13.5 & $<1.7$ & 2.6 & 0.8 & 5.2 & 2.0 & 11.4 & 12.9 & 0.8 & na & 129 & 3.8 & na & 9.5 \\
\hline $\begin{array}{l}\text { Kings Hill, } \\
\text { Mont. }\end{array}$ & $3 / 11 / 1998$ & E & -7.4 & 4.1 & 5.19 & 6.5 & 4.5 & $<1.7$ & $<1.0$ & 0.5 & 5.2 & $<1.0$ & 5.2 & 7.4 & 0.6 & na & 115 & 6.5 & na & 13.8 \\
\hline $\begin{array}{c}\text { Lake Irene, } \\
\text { Colo. }\end{array}$ & $3 / 24 / 1998$ & E & -18.1 & 6.4 & 4.79 & 16.2 & $<3.1$ & $<1.7$ & $<1.0$ & 1.8 & 2.3 & $<1.0$ & 6.5 & 10.7 & 0.4 & na & 146 & 7.0 & na & 8.5 \\
\hline $\begin{array}{l}\text { Lewis Lake Di- } \\
\text { vide, Wyo. }\end{array}$ & 3/4/1998 & E & -8.0 & 3.3 & 5.15 & 7.1 & $<3.1$ & $<1.7$ & $<1.0$ & $<0.4$ & 5.9 & 1.1 & 3.5 & 6.3 & 0.4 & na & 221 & 7.1 & na & 8.3 \\
\hline $\begin{array}{l}\text { Lionshead, } \\
\text { Mont. }\end{array}$ & 3/7/1998 & $\mathrm{E}$ & -9.7 & 5.3 & 5.03 & 9.3 & $<3.1$ & $<1.7$ & 1.3 & $<0.4$ & 10.5 & 2.3 & 7.1 & 10.9 & 0.5 & na & 144 & 5.5 & na & 2.1 \\
\hline $\begin{array}{l}\text { Little Brush } \\
\text { Creek, Utah }\end{array}$ & $3 / 27 / 1998$ & E & 0.9 & 6.1 & 5.77 & 1.7 & 16.5 & 4.1 & 7.8 & 1.5 & 11.3 & 7.1 & 9.6 & 14.3 & 1.1 & na & 107 & 6.4 & na & 14.8 \\
\hline $\begin{array}{l}\text { Loch Vale For- } \\
\text { est, Colo. }\end{array}$ & 4/7/1998 & $\mathrm{E}$ & -9.2 & 6.5 & 5.11 & 7.8 & 4.5 & $<1.7$ & $<1.0$ & $<0.4$ & 4.3 & $<1.0$ & 8.1 & 11.9 & 0.6 & 91 & 263 & na & na & -9.2 \\
\hline $\begin{array}{l}\text { Loveland Pass, } \\
\text { Colo. }\end{array}$ & $4 / 17 / 1998$ & E & -6.6 & 3.8 & 5.17 & 6.8 & 7.5 & $<1.7$ & 2.6 & $<0.4$ & 1.8 & 2.3 & 4.8 & 8.8 & 0.5 & na & 168 & 5.5 & na & 8.3 \\
\hline
\end{tabular}


[E, depth-integrated environmental; ANC, acid neutralization capacity; $\mu \mathrm{eq} / \mathrm{L}$, microequivalents per liter; $\mu \mathrm{S} / \mathrm{cm}$, microsiemens per centimeter; $\mathrm{SC}$, specific conductance at $25^{\circ} \mathrm{C} ; \mathrm{pH}$ values in standard units; $\mathrm{H}$, hydrogen; Ca, calcium; Mg, magnesium; $\mathrm{Na}$, sodium; $\mathrm{K}$, potassium; $\mathrm{NH}_{4}$, ammonium; $\mathrm{Cl}$, chloride; $\mathrm{SO}_{4}$, sulfate; $\mathrm{NO}_{3}$, nitrate; DOC, dissolved organic carbon; mg/L, milligrams per liter; $\mathrm{SWE}$, snow/water equivalent; cm, centimeters; $\delta^{34} \mathrm{~S}$, stable sulfur isotope ratio $\left({ }^{34} \mathrm{~S} /{ }^{22} \mathrm{~S}\right) ; \mathrm{Hg}$, mercury; ng/L, nanograms per liter; na, not analyzed; <, below reporting limits; NP, National Park; \%, percent]

\begin{tabular}{|c|c|c|c|c|c|c|c|c|c|c|c|c|c|c|c|c|c|c|c|c|}
\hline Site name & $\begin{array}{l}\text { Sample } \\
\text { date }\end{array}$ & $\begin{array}{l}\text { Sample } \\
\text { type }\end{array}$ & $\begin{array}{c}\text { ANC } \\
\text { ( } \mu \mathrm{eq} / \mathrm{L})\end{array}$ & $\begin{array}{c}S C \\
(\mu \mathrm{S} / \mathrm{cm})\end{array}$ & $\mathrm{pH}$ & 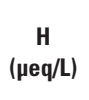 & $\begin{array}{c}\mathrm{Ca} \\
(\mu \mathrm{eq} / \mathrm{L})\end{array}$ & $\begin{array}{c}M g \\
(\mu \mathrm{eq} / \mathrm{L})\end{array}$ & $\underset{(\mu \mathrm{eq} / \mathrm{L})}{\mathrm{Na}}$ & $\begin{array}{c}K \\
\text { ( } \mu \text { eq/L) }\end{array}$ & $\begin{array}{c}\mathrm{NH}_{4} \\
(\mu \mathrm{eq} / \mathrm{L})\end{array}$ & $\underset{(\mu e q / L)}{C l}$ & $\begin{array}{c}\mathrm{SO}_{4} \\
(\mu \mathrm{eq} / \mathrm{L})\end{array}$ & $\begin{array}{c}\mathrm{NO}_{3} \\
(\mu \mathrm{eq} / \mathrm{L})\end{array}$ & $\begin{array}{l}\text { DOC } \\
(\mu \mathrm{g} / \mathrm{L})\end{array}$ & $\begin{array}{l}\text { SWE } \\
\text { (cm) }\end{array}$ & $\begin{array}{l}\text { Snow } \\
\text { depth } \\
\text { (cm) }\end{array}$ & $\begin{array}{c}\delta^{34} \mathrm{~S} \\
\text { (per mil) }\end{array}$ & $\underset{\text { (ng/L) }}{\mathrm{Hg}}$ & $\begin{array}{c}\text { Ionic } \\
\text { balance } \\
(\%)\end{array}$ \\
\hline $\begin{array}{c}\text { Lynx Pass, } \\
\text { Colo. }\end{array}$ & $3 / 11 / 1998$ & E & -20.8 & 8.3 & 4.71 & 19.5 & 3.5 & $<1.7$ & $<1.0$ & $<0.4$ & 3.0 & 1.1 & 6.9 & 15.0 & 0.5 & na & 122 & 6.7 & na & 6.1 \\
\hline $\begin{array}{c}\text { Molas Lake, } \\
\text { Colo. }\end{array}$ & $3 / 19 / 1998$ & E & -7.1 & 4.0 & 5.15 & 7.1 & 9.0 & $<1.7$ & $<1.0$ & $<0.4$ & 2.4 & 1.1 & 6.2 & 9.1 & $<0.4$ & na & 140 & 4.2 & na & 5.7 \\
\hline $\begin{array}{l}\text { Monarch Pass, } \\
\text { Colo. }\end{array}$ & 3/17/1998 & E & -10.9 & 5.3 & 4.97 & 10.7 & 5.5 & $<1.7$ & $<1.0$ & $<0.4$ & 3.1 & 1.4 & 6.9 & 10.4 & 0.7 & na & 130 & 5.4 & na & 1.6 \\
\hline $\begin{array}{l}\text { Monida Pass, } \\
\text { Mont. }\end{array}$ & $3 / 5 / 1998$ & E & -6.2 & 4.8 & 5.20 & 6.3 & 4.5 & $<1.7$ & 1.3 & 1.3 & 12.3 & 3.1 & 6.0 & 11.4 & 0.7 & na & 67 & na & na & 11.0 \\
\hline $\begin{array}{l}\text { Mount Bel- } \\
\text { mont, Mont. }\end{array}$ & $3 / 11 / 1998$ & E & -6.4 & 5.0 & 5.07 & 8.5 & 5.5 & $<1.7$ & $<1.0$ & 2.1 & 3.2 & 1.4 & 4.8 & 8.0 & 1.3 & na & 86 & na & na & 15.3 \\
\hline $\begin{array}{l}\text { Niwot Snotel, } \\
\text { Colo. }\end{array}$ & $3 / 20 / 1998$ & E & -12.0 & 6.1 & 4.94 & 11.5 & $<3.1$ & $<1.7$ & $<1.0$ & $<0.4$ & 8.4 & $<1.0$ & 7.7 & 11.2 & 0.5 & na & 151 & 6.1 & na & 2.3 \\
\hline $\begin{array}{l}\text { Noisy Basin, } \\
\text { Mont. }\end{array}$ & $3 / 12 / 1998$ & E & -6.9 & 3.5 & 5.18 & 6.6 & 3.5 & $<1.7$ & 1.3 & $<0.4$ & 3.8 & 1.1 & 4.2 & 5.7 & 0.5 & na & 307 & 6.3 & na & 15.7 \\
\hline $\begin{array}{c}\text { Old Battle, } \\
\text { Wyo. }\end{array}$ & $3 / 24 / 1998$ & E & -18.7 & 7.0 & 4.77 & 17.0 & 4.0 & $<1.7$ & 1.3 & $<0.4$ & 3.9 & 1.1 & 9.6 & 11.6 & 0.5 & na & 231 & 7.1 & na & 7.8 \\
\hline $\begin{array}{l}\text { Old Faithful } \\
\text { Crew Cor- } \\
\text { rals, Wyo. }\end{array}$ & $3 / 6 / 1998$ & E & -6.2 & 3.9 & 5.23 & 5.9 & $<3.1$ & $<1.7$ & 1.3 & $<0.4$ & 9.2 & 1.7 & 4.0 & 9.7 & 0.5 & na & 85 & na & na & 3.1 \\
\hline $\begin{array}{l}\text { Old Faithful } \\
\text { East Lot, } \\
\text { Wyo. }\end{array}$ & $3 / 6 / 1998$ & E & -9.1 & 4.7 & 5.06 & 8.7 & $<3.1$ & $<1.7$ & 3.5 & $<0.4$ & 5.7 & 3.4 & 4.2 & 9.5 & 0.5 & na & 86 & na & na & 2.3 \\
\hline $\begin{array}{l}\text { Old Faithful } \\
\text { Fire Road, } \\
\text { Wyo. }\end{array}$ & $3 / 6 / 1998$ & E & -8.6 & 4.2 & 5.07 & 8.5 & $<3.1$ & $<1.7$ & 1.7 & $<0.4$ & 6.2 & 2.0 & 4.4 & 9.4 & 0.6 & na & 86 & na & na & 2.3 \\
\hline $\begin{array}{l}\text { Old Faithful (in } \\
\text { road), Wyo. }\end{array}$ & 3/6/1998 & E & -9.7 & 4.5 & 4.94 & 11.5 & 13.0 & 2.5 & 5.7 & 1.8 & 21.1 & 7.3 & 19.6 & 10.6 & 5.9 & na & 12 & na & na & 19.3 \\
\hline $\begin{array}{l}\text { Biscuit Basin } \\
\text { (in road), } \\
\text { Wyo. }\end{array}$ & $3 / 6 / 1998$ & E & -6.8 & 4.4 & 5.15 & 7.1 & $<3.1$ & $<1.7$ & 1.7 & $<0.4$ & 7.7 & 1.7 & 4.4 & 8.6 & 0.5 & na & 29 & na & na & 5.8 \\
\hline $\begin{array}{l}\text { Jackson Hole } \\
\text { Airport, } \\
\text { Wyo. }\end{array}$ & $3 / 19 / 1998$ & E & -9.6 & 4.9 & 5.00 & 10.0 & 5.0 & $<1.7$ & $<1.0$ & 0.5 & 2.3 & 2.0 & 3.5 & 8.4 & 3.6 & na & 70 & na & na & 12.2 \\
\hline $\begin{array}{l}\text { Lewis Lake } \\
\text { Divide (in } \\
\text { road), Wyo. }\end{array}$ & $3 / 4 / 1998$ & E & -5.6 & 3.4 & 5.17 & 6.8 & $<3.1$ & $<1.7$ & $<1.0$ & $<0.4$ & 7.8 & 1.1 & 4.6 & 5.3 & 0.8 & na & 132 & na & na & 13.6 \\
\hline
\end{tabular}




\section{Table 7. Selected chemical and physical data for Rocky Mountain snowpack samples for 1998.—Continued}

[E, depth-integrated environmental; $\mathrm{ANC}$, acid neutralization capacity; $\mu \mathrm{eq} / \mathrm{L}$, microequivalents per liter; $\mu \mathrm{S} / \mathrm{cm}$, microsiemens per centimeter; $\mathrm{SC}$, specific conductance at $25^{\circ} \mathrm{C}$; $\mathrm{pH}$ values in standard units; $\mathrm{H}$, hydrogen; $\mathrm{Ca}$, calcium; $\mathrm{Mg}$, magnesium; $\mathrm{Na}$, sodium; $\mathrm{K}$, potassium; $\mathrm{NH}_{4}$, ammonium; $\mathrm{Cl}$, chloride; $\mathrm{SO}_{4}$, sulfate; $\mathrm{NO}_{3}$, nitrate; $\mathrm{DOC}$, dissolved organic carbon; mg/L, milligrams per liter; $\mathrm{SWE}$, snow/water equivalent; cm, centimeters; $\delta^{34} \mathrm{~S}$, stable sulfur isotope ratio $\left({ }^{34} \mathrm{~S} /{ }^{32} \mathrm{~S}\right) ; \mathrm{Hg}$, mercury; ng/L, nanograms per liter; na, not analyzed; <, below reporting limits; NP, National Park; \%, percent]

\begin{tabular}{|c|c|c|c|c|c|c|c|c|c|c|c|c|c|c|c|c|c|c|c|c|}
\hline Site name & $\begin{array}{l}\text { Sample } \\
\text { date }\end{array}$ & $\begin{array}{l}\text { Sample } \\
\text { type }\end{array}$ & $\begin{array}{c}\text { ANC } \\
\text { (peq/L) }\end{array}$ & $\begin{array}{c}S C \\
(\mu \mathrm{S} / \mathrm{cm})\end{array}$ & $\mathrm{pH}$ & $\begin{array}{c}H \\
(\mu e q / L)\end{array}$ & $\begin{array}{c}\mathrm{Ca} \\
(\mu \mathrm{eq} / \mathrm{L})\end{array}$ & $\begin{array}{c}\mathrm{Mg} \\
(\mu \mathrm{eq} / \mathrm{L})\end{array}$ & $\underset{(\mu \mathrm{Na} / \mathrm{L})}{\mathrm{Na}}$ & 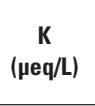 & $\begin{array}{c}\mathrm{NH}_{4} \\
(\mu \mathrm{eq} / \mathrm{L})\end{array}$ & $\begin{array}{c}C l \\
(\mu e q / L)\end{array}$ & $\begin{array}{c}\mathrm{SO}_{4} \\
(\mu \mathrm{eq} / \mathrm{L})\end{array}$ & $\begin{array}{c}\mathrm{NO}_{3} \\
(\mu \mathrm{eq} / \mathrm{L})\end{array}$ & $\begin{array}{c}\text { DOC } \\
(\mu g / L)\end{array}$ & $\begin{array}{l}\text { SWE } \\
(\mathrm{cm})\end{array}$ & $\begin{array}{l}\text { Snow } \\
\text { depth } \\
(\mathrm{cm})\end{array}$ & $\begin{array}{c}\delta^{34} \mathbf{S} \\
\text { (per mil) }\end{array}$ & $\underset{(\mathbf{n g} / \mathbf{L})}{\mathrm{Hg}}$ & $\begin{array}{c}\begin{array}{c}\text { lonic } \\
\text { balance }\end{array} \\
(\%)\end{array}$ \\
\hline $\begin{array}{l}\text { Old Faithful } \\
\text { 1000m, } \\
\text { Wyo. }\end{array}$ & 3/6/1998 & E & -8.3 & 4.4 & 5.09 & 8.1 & $<3.1$ & $<1.7$ & $<1.0$ & $<0.4$ & 6.4 & 1.7 & 4.0 & 8.4 & 0.4 & na & 118 & na & na & 2.0 \\
\hline $\begin{array}{l}\text { Phantom } \\
\text { Valley, Colo. }\end{array}$ & $3 / 23 / 1998$ & E & -21.4 & 7.7 & 4.68 & 20.9 & $<3.1$ & $<1.7$ & $<1.0$ & $<0.4$ & 2.6 & $<1.0$ & 6.9 & 11.9 & 0.7 & na & 71 & 7.2 & na & 11.3 \\
\hline $\begin{array}{l}\text { Rabbit Ears 1, } \\
\text { Colo. }\end{array}$ & 4/1/1998 & E & -19.1 & 8.3 & 4.71 & 19.5 & 3.5 & $<1.7$ & $<1.0$ & $<0.4$ & 5.1 & $<1.0$ & 9.6 & 11.9 & 0.6 & 70 & 226 & 7.7 & na & 13.4 \\
\hline $\begin{array}{l}\text { Rabbit Ears 2, } \\
\text { Colo. }\end{array}$ & 4/1/1998 & E & -20.9 & 8.7 & 4.69 & 20.4 & $<3.1$ & $<1.7$ & $<1.0$ & $<0.4$ & 4.5 & $<1.0$ & 10.0 & 11.7 & 0.5 & na & 238 & 8.1 & na & 6.8 \\
\hline $\begin{array}{l}\text { Red Mountain, } \\
\text { Mont. }\end{array}$ & 3/17/1998 & E & -7.1 & 3.4 & 5.10 & 7.9 & $<3.1$ & $<1.7$ & 2.2 & $<0.4$ & 3.8 & $<1.0$ & 3.5 & 5.5 & 0.6 & na & 217 & 7.0 & na & 21.1 \\
\hline $\begin{array}{l}\text { Red Mountain } \\
\text { Pass, Colo. }\end{array}$ & $3 / 19 / 1998$ & E & -7.0 & 4.3 & 5.12 & 7.6 & 9.5 & $<1.7$ & 2.6 & $<0.4$ & 2.1 & $<1.0$ & 6.2 & 9.6 & 0.5 & na & 190 & 4.7 & na & 15.9 \\
\hline $\begin{array}{l}\text { Rendezvous } \\
\text { Mountain, } \\
\text { Wyo. }\end{array}$ & $3 / 19 / 1998$ & E & -3.5 & 2.6 & 5.52 & 3.0 & 4.5 & 3.3 & 1.7 & 0.8 & 3.0 & $<1.0$ & 4.4 & 4.9 & 0.7 & na & 172 & 7.2 & na & 27.7 \\
\hline $\begin{array}{l}\text { Ripple Creek } \\
\text { Pass, Colo. }\end{array}$ & $3 / 31 / 1998$ & E & -12.5 & 6.3 & 4.88 & 13.2 & 5.5 & $<1.7$ & 1.7 & $<0.4$ & 3.0 & 1.1 & 7.7 & 10.0 & 0.5 & na & 189 & 5.4 & na & 10.8 \\
\hline $\begin{array}{l}\text { Slumgullion } \\
\text { Pass, Colo. }\end{array}$ & $3 / 18 / 1998$ & E & -8.5 & 4.8 & 5.06 & 8.7 & 7.5 & $<1.7$ & 3.5 & 0.8 & 1.9 & 2.5 & 6.7 & 8.2 & 0.6 & na & 92 & 3.7 & na & 12.6 \\
\hline $\begin{array}{l}\text { Snow Bowl, } \\
\text { Mont. }\end{array}$ & 3/14/1998 & E & -6.9 & 3.0 & 5.12 & 7.6 & $<3.1$ & $<1.7$ & 3.9 & $<0.4$ & 2.0 & $<1.0$ & 3.1 & 4.2 & 0.6 & na & 212 & 6.1 & na & 29.8 \\
\hline $\begin{array}{l}\text { South Pass, } \\
\text { Wyo. }\end{array}$ & $3 / 23 / 1998$ & E & -13.2 & 6.3 & 4.87 & 13.5 & 4.0 & $<1.7$ & 2.2 & $<0.4$ & 3.0 & $<1.0$ & 7.5 & 9.2 & 0.5 & na & 105 & 10.2 & na & 15.1 \\
\hline $\begin{array}{l}\text { Spring Gulch, } \\
\text { Mont. }\end{array}$ & $3 / 10 / 1998$ & E & -4.7 & 3.5 & 5.22 & 6.0 & 4.0 & $<1.7$ & 1.3 & $<0.4$ & 6.9 & $<1.0$ & 5.4 & 8.0 & 0.6 & na & 80 & 5.5 & na & 15.1 \\
\hline $\begin{array}{l}\text { Sunlight Creek, } \\
\text { Mont. }\end{array}$ & 3/9/1998 & E & -6.9 & 3.3 & 5.13 & 7.4 & $<3.1$ & $<1.7$ & 1.3 & $<0.4$ & 3.9 & $<1.0$ & 3.5 & 6.2 & 0.4 & na & 106 & na & na & 12.9 \\
\hline $\begin{array}{l}\text { Sunlight Peak, } \\
\text { Colo. }\end{array}$ & 3/30/1998 & E & -11.3 & 5.3 & 4.96 & 11.0 & 4.5 & $<1.7$ & 1.7 & $<0.4$ & 4.3 & 1.1 & 7.3 & 9.2 & 0.4 & na & 175 & 5.5 & na & 9.9 \\
\hline $\begin{array}{l}\text { Sylvan Lake, } \\
\text { Wyo. }\end{array}$ & $3 / 3 / 1998$ & E & -8.8 & 3.6 & 5.20 & 6.3 & $<3.1$ & $<1.7$ & 1.7 & $<0.4$ & 4.4 & 1.1 & 3.3 & 5.7 & 0.4 & na & 147 & 5.5 & na & 9.9 \\
\hline $\begin{array}{l}\text { Sylvan Lake (in } \\
\text { road), Wyo. }\end{array}$ & 3/3/1998 & E & -8.8 & 4.0 & 5.19 & 6.5 & $<3.1$ & $<1.7$ & 2.6 & $<0.4$ & 4.0 & 1.4 & 4.8 & 6.2 & 0.6 & na & 96 & na & na & 2.7 \\
\hline $\begin{array}{l}\text { Teton Pass, } \\
\text { Wyo. }\end{array}$ & 3/17/1998 & E & -6.0 & 3.8 & 5.45 & 3.5 & 7.0 & 2.5 & 2.6 & 0.5 & 5.3 & 2.3 & 4.8 & 8.1 & 0.4 & na & 124 & 5.9 & na & 17.1 \\
\hline
\end{tabular}


[E, depth-integrated environmental; ANC, acid neutralization capacity; $\mu \mathrm{eq} / \mathrm{L}$, microequivalents per liter; $\mu \mathrm{S} / \mathrm{cm}$, microsiemens per centimeter; $\mathrm{SC}$, specific conductance at $25^{\circ} \mathrm{C} ; \mathrm{pH}$ values in standard units; $\mathrm{H}$, hydrogen; Ca, calcium; Mg, magnesium; $\mathrm{Na}$, sodium; $\mathrm{K}$, potassium; $\mathrm{NH}_{4}$, ammonium; $\mathrm{Cl}$, chloride; $\mathrm{SO}_{4}$, sulfate; $\mathrm{NO}_{3}$, nitrate; DOC, dissolved organic carbon; mg/L, milligrams per liter; $\mathrm{SWE}$, snow/water equivalent; cm, centimeters; $\delta^{34} \mathrm{~S}$, stable sulfur isotope ratio $\left({ }^{34} \mathrm{~S} /{ }^{22} \mathrm{~S}\right) ; \mathrm{Hg}$, mercury; ng/L, nanograms per liter; na, not analyzed; <, below reporting limits; NP, National Park; \%, percent]

\begin{tabular}{|c|c|c|c|c|c|c|c|c|c|c|c|c|c|c|c|c|c|c|c|c|}
\hline Site name & $\begin{array}{l}\text { Sample } \\
\text { date }\end{array}$ & $\begin{array}{l}\text { Sample } \\
\text { type }\end{array}$ & 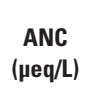 & $\begin{array}{c}\mathrm{SC} \\
(\mu \mathrm{S} / \mathrm{cm})\end{array}$ & pH & 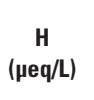 & 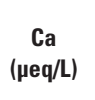 & $\underset{\text { (peq/L) }}{M g}$ & 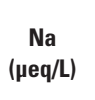 & $\begin{array}{c}K \\
\text { ( } \mu \mathrm{eq} / L \text { ) }\end{array}$ & $\begin{array}{c}\mathrm{NH}_{4} \\
\text { ( } \mu \mathrm{eq} / \mathrm{L} \text { ) }\end{array}$ & 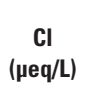 & $\begin{array}{c}\mathrm{SO}_{4} \\
(\mu \mathrm{eq} / \mathrm{L})\end{array}$ & $\begin{array}{c}\mathrm{NO}_{3} \\
(\mu \mathrm{eq} / \mathrm{L})\end{array}$ & $\begin{array}{l}\text { DOC } \\
(\mu \mathrm{g} / \mathrm{L})\end{array}$ & $\begin{array}{l}\text { SWE } \\
\text { (cm) }\end{array}$ & $\begin{array}{l}\text { Snow } \\
\text { depth } \\
(\mathrm{cm})\end{array}$ & $\begin{array}{c}\delta^{34} \mathbf{S} \\
\text { (per mil) }\end{array}$ & $\underset{(\mathrm{ng} / \mathrm{L})}{\mathrm{Hg}}$ & $\begin{array}{c}\text { lonic } \\
\text { balance } \\
(\%)\end{array}$ \\
\hline $\begin{array}{l}\text { Togwotee Pass, } \\
\text { Wyo. }\end{array}$ & $3 / 24 / 1998$ & $\mathrm{E}$ & -8.7 & 2.9 & 5.26 & 5.5 & $<3.1$ & $<1.7$ & 3.0 & $<0.4$ & 2.3 & 1.1 & 3.5 & 4.7 & 0.4 & na & 179 & 6.9 & na & 7.3 \\
\hline $\begin{array}{l}\text { Tower Falls, } \\
\text { Wyo. }\end{array}$ & $3 / 10 / 1998$ & $\mathrm{E}$ & -10.4 & 3.7 & 5.10 & 7.9 & $<3.1$ & $<1.7$ & $<1.0$ & $<0.4$ & 4.3 & 1.4 & 2.7 & 7.4 & 0.5 & na & 63 & 4.3 & na & 3.2 \\
\hline $\begin{array}{l}\text { Trappers Lake, } \\
\text { Colo. }\end{array}$ & $3 / 31 / 1998$ & $\mathrm{E}$ & -13.4 & 4.7 & 4.97 & 10.7 & 3.5 & $<1.7$ & 1.7 & $<0.4$ & 2.3 & $<1.0$ & 4.8 & 9.2 & 0.4 & na & 174 & 4.9 & na & 13.3 \\
\hline $\begin{array}{l}\text { Trial Lake, } \\
\text { Utah }\end{array}$ & $3 / 26 / 1998$ & $\mathrm{E}$ & -4.7 & 2.9 & 5.46 & 3.5 & 4.5 & $<1.7$ & 4.4 & $<0.4$ & 1.8 & 2.0 & 5.0 & 6.0 & $<0.4$ & na & 145 & 10.9 & na & 4.2 \\
\hline $\begin{array}{l}\text { Twenty-one } \\
\text { Mile, Mont. }\end{array}$ & 3/7/1998 & E & -11.2 & 5.1 & 5.06 & 8.7 & $<3.1$ & $<1.7$ & 2.6 & $<0.4$ & 7.0 & 2.3 & 5.4 & 9.7 & 0.5 & na & 130 & 4.8 & na & 2.6 \\
\hline $\begin{array}{l}\text { University } \\
\text { Camp, Colo. }\end{array}$ & 3/13/1998 & E & -14.4 & 5.9 & 4.95 & 11.2 & 5.0 & $<1.7$ & 2.2 & 1.0 & 6.0 & 1.1 & 8.5 & 11.2 & 0.7 & na & 208 & 6.3 & na & 9.8 \\
\hline $\begin{array}{l}\text { West Yellow- } \\
\text { stone, Mont. }\end{array}$ & $3 / 5 / 1998$ & E & -12.3 & 5.5 & 5.00 & 10.0 & $<3.1$ & $<1.7$ & 3.0 & $<0.4$ & 9.1 & 2.5 & 6.0 & 13.1 & 0.8 & na & 73 & 5.8 & na & 1.1 \\
\hline $\begin{array}{l}\text { West Yellow- } \\
\text { stone } \\
1,000 \mathrm{~m} \\
\text { Mont. }\end{array}$ & $3 / 5 / 1998$ & E & -13.6 & 6.3 & 4.85 & 14.1 & $<3.1$ & $<1.7$ & 3.0 & $<0.4$ & 8.0 & 2.5 & 6.0 & 12.4 & $<0.4$ & na & 85 & na & na & 9.2 \\
\hline $\begin{array}{l}\text { West Yellow- } \\
\text { stone 8km, } \\
\text { Wyo. }\end{array}$ & $3 / 6 / 1998$ & E & -11.5 & 5.1 & 4.94 & 11.5 & $<3.1$ & $<1.7$ & 1.7 & $<0.4$ & 6.2 & 1.7 & 4.2 & 11.2 & 0.4 & na & 64 & na & na & 6.5 \\
\hline $\begin{array}{l}\text { West Yellow- } \\
\text { stone 8km } \\
\text { (in road), } \\
\text { Wyo. }\end{array}$ & $3 / 6 / 1998$ & E & -7.3 & 9.2 & 5.01 & 9.8 & 11.0 & 2.5 & 9.1 & 1.3 & 24.5 & 8.7 & 17.5 & 14.7 & 3.7 & na & 20 & na & na & 17.4 \\
\hline $\begin{array}{l}\text { Willow Creek, } \\
\text { Wyo. }\end{array}$ & $3 / 25 / 1998$ & E & -10.3 & 4.0 & 4.97 & 10.7 & 3.5 & $<1.7$ & 2.6 & $<0.4$ & 5.4 & 1.7 & 5.8 & 7.8 & $<0.4$ & na & 188 & 5.7 & na & 18.3 \\
\hline $\begin{array}{l}\text { Wolf Creek } \\
\text { Pass, Colo. }\end{array}$ & 3/18/1998 & E & -6.5 & 4.4 & 5.22 & 6.0 & 12.5 & 2.5 & 3.5 & 0.5 & 3.8 & 1.7 & 9.2 & 9.5 & 0.4 & na & 175 & 3.5 & na & 17.1 \\
\hline $\begin{array}{l}\text { West Yellow- } \\
\text { stone (in } \\
\text { road), Mont. }\end{array}$ & $3 / 5 / 1998$ & E & -7.6 & 10.5 & 4.99 & 10.2 & 13.0 & 3.3 & 13.9 & 2.1 & 23.0 & 12.4 & 20.6 & 15.3 & 3.7 & na & 46 & 5.2 & na & 15.0 \\
\hline
\end{tabular}




\section{Table 8. Selected chemical and physical data for Rocky Mountain snowpack samples for 1999.}

[E, depth-integrated environmental; ANC, acid neutralization capacity; $\mu \mathrm{eq} / \mathrm{L}$, microequivalents per liter; $\mu \mathrm{S} / \mathrm{cm}$, microsiemens per centimeter; $\mathrm{SC}$, specific conductance at $25^{\circ} \mathrm{C} ; \mathrm{pH}$ values in standard units; $\mathrm{H}$, hydrogen; $\mathrm{Ca}$, calcium; $\mathrm{Mg}$, magnesium; $\mathrm{Na}$, sodium; $\mathrm{K}$, potassium; $\mathrm{NH}_{4}$, ammonium; $\mathrm{Cl}$, chloride; $\mathrm{SO}_{4}$, sulfate; $\mathrm{NO}_{3}$, nitrate; $\mathrm{DOC}$, dissolved organic carbon; mg/L, milligrams per liter; $\mathrm{SWE}$, snow/water equivalent; cm, centimeters; $\delta^{34} \mathrm{~S}$, stable sulfur isotope ratio $\left({ }^{34} \mathrm{~S} /{ }^{22} \mathrm{~S}\right) ; \mathrm{Hg}$, mercury; ng/L, nanograms per liter; na, not analyzed; <, below reporting limits; NP, National Park; \%, percent]

\begin{tabular}{|c|c|c|c|c|c|c|c|c|c|c|c|c|c|c|c|c|c|c|c|c|}
\hline Site name & $\begin{array}{l}\text { Sample } \\
\text { date }\end{array}$ & $\begin{array}{c}\text { Sample } \\
\text { type }\end{array}$ & $\begin{array}{c}\text { ANC } \\
(\mu e q / L)\end{array}$ & $\begin{array}{c}S C \\
(\mu \mathrm{S} / \mathrm{cm})\end{array}$ & $\mathrm{pH}$ & 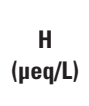 & $\begin{array}{c}\mathrm{Ca} \\
(\mu \mathrm{eq} / \mathrm{L})\end{array}$ & $\begin{array}{c}\mathrm{Mg} \\
(\mu \mathrm{eq} / \mathrm{L})\end{array}$ & $\underset{\text { (peq/L) }}{\mathrm{Na}}$ & $\begin{array}{c}K \\
(\mu e q / L)\end{array}$ & $\begin{array}{c}\mathrm{NH}_{4} \\
\text { (peq/L) }\end{array}$ & 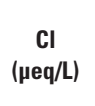 & $\begin{array}{c}\mathrm{SO}_{4} \\
(\mu \mathrm{eq} / \mathrm{L})\end{array}$ & $\begin{array}{c}\mathrm{NO}_{3} \\
(\mu \mathrm{eq} / \mathrm{L})\end{array}$ & $\begin{array}{l}\text { DOC } \\
(\mu \mathrm{g} / \mathrm{L})\end{array}$ & $\begin{array}{l}\text { SWE } \\
\text { (cm) }\end{array}$ & $\begin{array}{l}\text { Snow } \\
\text { depth } \\
\text { (cm) }\end{array}$ & $\begin{array}{c}\delta^{34} \mathbf{S} \\
\text { (per mil) }\end{array}$ & $\underset{(\mathrm{ng} / \mathrm{L})}{\mathrm{Hg}}$ & $\begin{array}{c}\text { Ionic } \\
\text { balance } \\
(\%)\end{array}$ \\
\hline $\begin{array}{l}\text { Apgar Look- } \\
\text { out, Mont. }\end{array}$ & $3 / 25 / 1999$ & $\mathrm{E}$ & -6.5 & 3.4 & 5.28 & 5.2 & $<3.1$ & $<1.7$ & 2.1 & 0.6 & 5.5 & 1.4 & 4.7 & 5.2 & 0.4 & 64 & 182 & 5.9 & na & 8.5 \\
\hline $\begin{array}{c}\text { Bear Lake, } \\
\text { Colo. }\end{array}$ & 4/14/1999 & E & -2.5 & 5.4 & 5.34 & 4.6 & 14.5 & 2.5 & 1.8 & 1.2 & 7.0 & 1.6 & 10.2 & 13.4 & 1.2 & na & 123 & na & na & 11.2 \\
\hline $\begin{array}{l}\text { Beaver Moun- } \\
\text { tain, Utah }\end{array}$ & 3/22/1999 & E & 5.3 & 4.8 & 5.96 & 1.1 & 13.5 & 2.5 & 8.2 & 0.8 & 8.9 & 5.3 & 8.0 & 9.2 & 0.5 & na & 204 & 8.3 & na & 11.5 \\
\hline $\begin{array}{l}\text { Berthoud Pass, } \\
\text { Colo. }\end{array}$ & 4/8/1999 & E & -4.8 & 5.4 & 5.27 & 5.4 & 11.0 & 2.5 & 3.9 & 2.4 & 4.4 & 3.4 & 5.7 & 10.4 & 1.8 & na & 170 & 6.1 & na & 20.3 \\
\hline $\begin{array}{l}\text { Big Mountain, } \\
\text { Mont. }\end{array}$ & 3/12/1999 & E & -7.4 & 4.4 & 5.18 & 6.6 & $<3.1$ & $<1.7$ & $<1.0$ & $<0.4$ & 4.0 & $<1.0$ & 3.2 & 4.0 & $<0.4$ & na & 365 & 5.7 & na & 18.5 \\
\hline $\begin{array}{l}\text { Big Sky, } \\
\text { Mont. }\end{array}$ & 3/17/1999 & E & -3.5 & 3.1 & 5.46 & 3.5 & 5.5 & $<1.7$ & $<1.0$ & $<0.4$ & 4.2 & 1.4 & 3.3 & 5.4 & $<0.4$ & na & 240 & 5.8 & na & 13.1 \\
\hline $\begin{array}{l}\text { Biscuit Basin, } \\
\text { Wyo. }\end{array}$ & 3/4/1999 & E & -2.0 & 4.1 & 5.47 & 3.4 & 4.5 & $<1.7$ & 2.7 & 0.5 & 9.4 & 1.6 & 5.5 & 6.2 & 0.6 & na & 159 & na & na & 21.1 \\
\hline $\begin{array}{l}\text { Brooklyn } \\
\text { Lake, Wyo. }\end{array}$ & 3/24/1999 & E & -7.7 & 5.2 & 5.20 & 6.3 & 8.0 & $<1.7$ & 1.8 & 0.5 & 4.9 & 1.6 & 7.4 & 9.8 & 0.4 & na & 240 & 7.0 & na & 6.5 \\
\hline $\begin{array}{c}\text { Brumley, } \\
\text { Colo. }\end{array}$ & 3/16/1999 & E & -4.4 & 4.8 & 5.35 & 4.5 & 10.5 & $<1.7$ & 1.5 & 0.9 & 3.7 & 1.3 & 5.5 & 9.6 & 0.5 & na & 96 & na & na & 12.3 \\
\hline $\begin{array}{l}\text { Buffalo Pass, } \\
\text { Colo. }\end{array}$ & 4/7/1999 & E & -6.5 & 6.0 & 5.21 & 6.2 & 13.0 & $<1.7$ & 2.5 & 0.5 & 5.5 & 1.7 & 12.1 & 12.8 & $<0.4$ & na & 340 & 7.3 & na & 2.1 \\
\hline $\begin{array}{l}\text { Cameron Pass, } \\
\text { Colo. }\end{array}$ & 4/8/1999 & E & -3.1 & 5.5 & 5.39 & 4.1 & 14.5 & 2.5 & 3.9 & 1.3 & 4.9 & 3.1 & 9.4 & 11.2 & 0.7 & na & 174 & 6.3 & na & 13.6 \\
\hline Canyon, Wyo. & $3 / 5 / 1999$ & E & -3.8 & 3.6 & 5.35 & 4.5 & $<3.1$ & $<1.7$ & $<1.0$ & $<0.4$ & 6.4 & 1.3 & 3.3 & 5.8 & $<0.4$ & na & 147 & na & na & 2.3 \\
\hline $\begin{array}{l}\text { Cement Gulch } \\
\text { Divide, } \\
\text { Mont. }\end{array}$ & 3/9/1999 & E & 1.0 & 3.8 & 5.75 & 1.8 & 11.0 & $<1.7$ & $<1.0$ & 0.5 & 6.0 & 1.2 & 5.6 & 7.0 & 0.5 & na & 73 & 6.9 & na & 13.0 \\
\hline $\begin{array}{l}\text { Chief Joseph } \\
\text { Pass, Mont. }\end{array}$ & 3/13/1999 & E & -4.6 & 3.1 & 5.34 & 4.6 & $<3.1$ & $<1.7$ & 1.2 & 0.5 & 3.4 & $<1.0$ & 2.1 & 2.9 & $<0.4$ & na & 179 & 5.1 & na & 32.0 \\
\hline $\begin{array}{c}\text { Daisy Pass, } \\
\text { Mont. }\end{array}$ & $3 / 8 / 1999$ & E & -6.1 & 3.6 & 5.30 & 5.0 & $<3.1$ & $<1.7$ & $<1.0$ & $<0.4$ & 4.6 & $<1.0$ & 2.9 & 5.0 & $<0.4$ & na & 328 & na & na & 9.9 \\
\hline $\begin{array}{l}\text { Deadman } \\
\text { Pass, Colo. }\end{array}$ & 3/23/1999 & E & -4.6 & 5.8 & 5.29 & 5.1 & 14.5 & 3.3 & 2.3 & 1.6 & 5.2 & 1.8 & 9.5 & 13.7 & 1.2 & na & 189 & 6.8 & na & 12.4 \\
\hline $\begin{array}{c}\text { Divide Peak, } \\
\text { Wyo. }\end{array}$ & 3/25/1999 & E & -6.7 & 6.3 & 5.20 & 6.3 & 14.0 & 2.5 & 3.5 & 0.7 & 6.3 & 2.0 & 10.9 & 15.8 & 0.6 & na & 198 & na & na & 7.2 \\
\hline $\begin{array}{c}\text { Dry Lake, } \\
\text { Colo. }\end{array}$ & 3/11/1999 & E & -10.2 & 7.6 & 4.99 & 10.2 & 13.0 & 2.5 & 4.1 & 0.6 & 6.3 & 1.9 & 13.2 & 16.7 & $<0.4$ & na & 127 & 8.4 & na & 7.0 \\
\hline
\end{tabular}


[E, depth-integrated environmental; $\mathrm{ANC}$, acid neutralization capacity; $\mu \mathrm{eq} / \mathrm{L}$, microequivalents per liter; $\mu \mathrm{S} / \mathrm{cm}$, microsiemens per centimeter; $\mathrm{SC}$, specific conductance at $25^{\circ} \mathrm{C} ; \mathrm{pH}$ values in standard units; $\mathrm{H}$, hydrogen; Ca, calcium; Mg, magnesium; $\mathrm{Na}$, sodium; $\mathrm{K}$, potassium; $\mathrm{NH}_{4}$, ammonium; $\mathrm{Cl}$, chloride; $\mathrm{SO}_{4}$, sulfate; $\mathrm{NO}_{3}$, nitrate; DOC, dissolved organic carbon; mg/L, milligrams per liter; $\mathrm{SWE}$, snow/water equivalent; cm, centimeters; $\delta^{34} \mathrm{~S}$, stable sulfur isotope ratio $\left({ }^{34} \mathrm{~S} /{ }^{22} \mathrm{~S}\right) ; \mathrm{Hg}$, mercury; ng/L, nanograms per liter; na, not analyzed; <, below reporting limits; NP, National Park; \%, percent]

\begin{tabular}{|c|c|c|c|c|c|c|c|c|c|c|c|c|c|c|c|c|c|c|c|c|}
\hline Site name & $\begin{array}{l}\text { Sample } \\
\text { date }\end{array}$ & $\begin{array}{c}\text { Sample } \\
\text { type }\end{array}$ & $\begin{array}{c}\text { ANC } \\
(\mu \mathrm{eq} / \mathrm{L})\end{array}$ & $\begin{array}{c}S C \\
(\mu \mathrm{S} / \mathrm{cm})\end{array}$ & pH & $\begin{array}{c}H \\
(\mu \mathrm{eq} / \mathrm{L})\end{array}$ & $\begin{array}{c}\mathrm{Ca} \\
(\mu \mathrm{eq} / \mathrm{L})\end{array}$ & $\begin{array}{c}\mathrm{Mg} \\
(\mu \mathrm{eq} / \mathrm{L})\end{array}$ & $\begin{array}{c}\mathrm{Na} \\
(\mu \mathrm{eq} / \mathrm{L})\end{array}$ & $\begin{array}{c}\mathbf{K} \\
(\mu \mathrm{eq} / L)\end{array}$ & $\underset{(\mu \mathrm{eq} / \mathrm{L})}{\mathrm{NH}_{4}}$ & $\begin{array}{c}C I \\
\text { (peq/L) }\end{array}$ & $\begin{array}{c}\mathrm{SO}_{4} \\
(\mu \mathrm{eq} / \mathrm{L})\end{array}$ & $\begin{array}{c}\mathrm{NO}_{3} \\
(\mu \mathrm{eq} / \mathrm{L})\end{array}$ & $\begin{array}{c}\text { DOC } \\
(\mu \mathrm{g} / \mathrm{L})\end{array}$ & $\begin{array}{l}\text { SWE } \\
\text { (cm) }\end{array}$ & $\begin{array}{l}\text { Snow } \\
\text { depth } \\
\text { (cm) }\end{array}$ & $\begin{array}{c}\delta^{34} \mathrm{~S} \\
\text { (per mil) }\end{array}$ & $\underset{\text { (ng/L) }}{\mathrm{Hg}}$ & $\begin{array}{c}\text { lonic } \\
\text { balance } \\
(\%)\end{array}$ \\
\hline $\begin{array}{l}\text { Dunckley } \\
\text { Pass, Colo. }\end{array}$ & 4/8/1999 & E & 31.2 & 7.1 & 6.57 & 0.3 & 45.4 & 4.1 & 2.4 & 3.0 & 5.8 & 1.8 & 8.1 & 13.7 & 1.3 & na & 145 & 4.6 & na & 5.3 \\
\hline $\begin{array}{l}\text { E1 (Glacier } \\
\text { NP), Mont. }\end{array}$ & $3 / 24 / 1999$ & E & -1.9 & 3.1 & 5.44 & 3.6 & 5.0 & $<1.7$ & 2.3 & 0.8 & 4.6 & 1.4 & 3.7 & 4.8 & 0.8 & 39 & 96 & 7.3 & na & 24.8 \\
\hline $\begin{array}{l}\text { E2 (Glacier } \\
\text { NP), Mont. }\end{array}$ & 4/1/1999 & E & -4.6 & 2.8 & 5.28 & 5.2 & $<3.1$ & $<1.7$ & $<1.0$ & $<0.4$ & 3.7 & $<1.0$ & 2.2 & 3.3 & 0.8 & 34 & 106 & na & na & 23.5 \\
\hline $\begin{array}{l}\text { E3 (Glacier } \\
\text { NP), Mont. }\end{array}$ & $3 / 28 / 1999$ & E & -6.7 & 3.5 & 5.19 & 6.5 & $<3.1$ & $<1.7$ & 1.2 & 0.5 & 3.8 & 1.0 & 4.0 & 4.1 & 0.7 & 66 & 174 & na & na & 12.8 \\
\hline $\begin{array}{l}\text { E4 (Glacier } \\
\text { NP), Mont. }\end{array}$ & 3/29/1999 & E & -5.7 & 3.6 & 5.22 & 6.0 & $<3.1$ & $<1.7$ & 3.1 & 1.8 & 4.1 & 1.3 & 4.2 & 4.6 & 1.1 & 99 & 256 & na & na & 19.4 \\
\hline $\begin{array}{l}\text { E5 (Glacier } \\
\text { NP), Mont. }\end{array}$ & $3 / 30 / 1999$ & E & -6.4 & 3.4 & 5.19 & 6.5 & $<3.1$ & $<1.7$ & $<1.0$ & 0.5 & 3.2 & $<1.0$ & 3.5 & 3.1 & 0.8 & 123 & 332 & na & na & 21.0 \\
\hline $\begin{array}{l}\text { E6 (Glacier } \\
\text { NP), Mont. }\end{array}$ & $3 / 31 / 1999$ & E & -7.5 & 3.9 & 5.16 & 6.9 & $<3.1$ & $<1.7$ & 1.2 & 0.8 & 3.8 & 1.2 & 4.1 & 4.3 & 1.2 & 112 & 288 & na & na & 14.3 \\
\hline $\begin{array}{l}\text { E7 (Glacier } \\
\text { NP), Mont. }\end{array}$ & 3/31/1999 & E & -1.4 & 3.7 & 5.39 & 4.1 & $<3.1$ & $<1.7$ & 1.9 & 3.8 & 3.1 & 1.6 & 4.1 & 3.7 & 2.8 & 83 & 229 & na & na & 14.9 \\
\hline $\begin{array}{c}\text { Elk River, } \\
\text { Colo. }\end{array}$ & $3 / 11 / 1999$ & E & -7.3 & 5.7 & 5.14 & 7.2 & 11.0 & $<1.7$ & 3.0 & 0.8 & 4.8 & 1.6 & 7.3 & 14.0 & $<0.4$ & na & 120 & 7.0 & na & 8.0 \\
\hline $\begin{array}{c}\text { Elkhart Park, } \\
\text { Wyo. }\end{array}$ & $3 / 23 / 1999$ & E & -6.8 & 4.4 & 5.23 & 5.9 & 5.0 & $<1.7$ & 1.3 & 0.9 & 4.9 & 1.5 & 5.7 & 7.0 & 0.4 & na & 126 & 8.2 & na & 12.0 \\
\hline $\begin{array}{l}\text { Four Mile } \\
\text { Meadow, } \\
\text { Wyo. }\end{array}$ & 3/19/1999 & E & -5.2 & 3.7 & 5.33 & 4.7 & 4.0 & $<1.7$ & 1.3 & 0.5 & 4.5 & 1.2 & 3.2 & 6.1 & 0.4 & na & 105 & na & na & 17.9 \\
\hline $\begin{array}{l}\text { Fremont Pass, } \\
\text { Colo. }\end{array}$ & 4/12/1999 & E & 3.1 & 4.0 & 5.79 & 1.6 & 17.0 & 2.5 & 1.7 & 1.1 & 3.2 & 1.4 & 5.2 & 9.3 & 0.6 & na & 129 & 5.2 & na & 17.6 \\
\hline $\begin{array}{l}\text { Gallegos Peak, } \\
\text { N. Mex. }\end{array}$ & 3/27/1999 & E & 5.0 & 5.6 & 5.88 & 1.3 & 22.5 & 2.5 & 2.1 & 2.3 & 6.6 & 1.5 & 10.9 & 12.7 & 1.1 & na & 118 & 5.4 & na & 10.5 \\
\hline $\begin{array}{l}\text { Garnet Can- } \\
\text { yon, Wyo. }\end{array}$ & $3 / 27 / 1999$ & E & -4.6 & 3.6 & 5.30 & 5.0 & $<3.1$ & $<1.7$ & 1.7 & $<0.4$ & 5.6 & 1.2 & 5.6 & 5.2 & $<0.4$ & na & 175 & 7.4 & na & 1.3 \\
\hline $\begin{array}{c}\text { Grand Mesa, } \\
\text { Colo. }\end{array}$ & 4/6/1999 & E & 28.4 & 7.5 & 6.46 & 0.3 & 44.4 & 4.1 & 3.4 & 2.3 & 7.8 & 2.0 & 12.3 & 11.5 & 1.3 & na & 167 & na & na & 7.1 \\
\hline $\begin{array}{l}\text { Granite Park, } \\
\text { Mont. }\end{array}$ & $3 / 31 / 1999$ & E & -6.7 & 3.7 & 5.20 & 6.3 & $<3.1$ & $<1.7$ & 1.4 & $<0.4$ & 4.1 & 1.4 & 3.9 & 4.9 & 0.7 & 170 & 404 & na & na & 7.6 \\
\hline $\begin{array}{l}\text { Granite Pass, } \\
\text { Mont. }\end{array}$ & 3/15/1999 & E & -5.3 & 2.6 & 5.32 & 4.8 & $<3.1$ & $<1.7$ & $<1.0$ & 0.6 & 1.2 & $<1.0$ & 1.7 & 1.5 & 0.4 & na & 292 & 7.7 & na & 34.8 \\
\hline
\end{tabular}




\section{Table 8. Selected chemical and physical data for Rocky Mountain snowpack samples for 1999.—Continued}

[E, depth-integrated environmental; $\mathrm{ANC}$, acid neutralization capacity; $\mu \mathrm{eq} / \mathrm{L}$, microequivalents per liter; $\mu \mathrm{S} / \mathrm{cm}$, microsiemens per centimeter; $\mathrm{SC}$, specific conductance at $25^{\circ} \mathrm{C}$; $\mathrm{pH}$ values in standard units; $\mathrm{H}$, hydrogen; $\mathrm{Ca}$, calcium; $\mathrm{Mg}$, magnesium; $\mathrm{Na}$, sodium; $\mathrm{K}$, potassium; $\mathrm{NH}_{4}$, ammonium; $\mathrm{Cl}$, chloride; $\mathrm{SO}_{4}$, sulfate; $\mathrm{NO}_{3}$, nitrate; DOC, dissolved organic carbon; mg/L, milligrams per liter; $\mathrm{SWE}$, snow/water equivalent; cm, centimeters; $\delta^{34} \mathrm{~S}$, stable sulfur isotope ratio $\left({ }^{34} \mathrm{~S} /{ }^{22} \mathrm{~S}\right) ; \mathrm{Hg}$, mercury; ng/L, nanograms per liter; na, not analyzed; <, below reporting limits; NP, National Park; \%, percent]

\begin{tabular}{|c|c|c|c|c|c|c|c|c|c|c|c|c|c|c|c|c|c|c|c|c|}
\hline Site name & $\begin{array}{l}\text { Sample } \\
\text { date }\end{array}$ & $\begin{array}{c}\text { Sample } \\
\text { type }\end{array}$ & $\begin{array}{c}\text { ANC } \\
\text { ( } \mu \text { eq/L) }\end{array}$ & $\begin{array}{c}\text { SC } \\
(\mu \mathrm{S} / \mathrm{cm})\end{array}$ & $\mathrm{pH}$ & 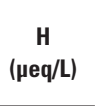 & $\underset{(\mu \mathrm{eq} / \mathrm{L})}{\mathrm{Ca}}$ & $\underset{\text { (peq/L) }}{M g}$ & $\begin{array}{c}\mathrm{Na} \\
(\mu \mathrm{eq} / \mathrm{L})\end{array}$ & $\underset{\text { (peq/L) }}{K}$ & $\begin{array}{c}\mathrm{NH}_{4} \\
(\mu \mathrm{eq} / \mathrm{L})\end{array}$ & $\underset{(\mu \mathrm{eq} / \mathrm{L})}{C I}$ & 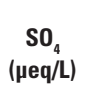 & 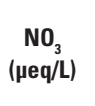 & $\begin{array}{c}\text { DOC } \\
(\mu \mathrm{g} / \mathrm{L})\end{array}$ & $\begin{array}{l}\text { SWE } \\
\text { (cm) }\end{array}$ & $\begin{array}{l}\text { Snow } \\
\text { depth } \\
\text { (cm) }\end{array}$ & $\begin{array}{c}\delta^{34} \mathbf{S} \\
\text { (per mil) }\end{array}$ & $\underset{(\mathrm{ng} / \mathrm{L})}{\mathrm{Hg}}$ & $\begin{array}{c}\text { lonic } \\
\text { balance } \\
(\%)\end{array}$ \\
\hline $\begin{array}{l}\text { Grassy Moun- } \\
\text { tain, Mont. }\end{array}$ & 3/8/1999 & E & 3.0 & 4.0 & 5.87 & 1.3 & 12.0 & $<1.7$ & $<1.0$ & 1.0 & 7.7 & 1.3 & 5.1 & 6.9 & 0.6 & na & 68 & na & na & 15.0 \\
\hline $\begin{array}{l}\text { Gypsum } \\
\text { Creek, } \\
\text { Wyo. }\end{array}$ & 3/23/1999 & E & -5.2 & 4.2 & 5.28 & 5.2 & 5.0 & $<1.7$ & 1.3 & 1.7 & 5.1 & 1.5 & 5.0 & 7.3 & 1.0 & na & 104 & na & na & 14.3 \\
\hline $\begin{array}{l}\text { Hogan Peak, } \\
\text { Colo. }\end{array}$ & 3/27/1999 & E & -11.2 & 7.0 & 5.00 & 10.0 & 9.0 & $<1.7$ & 2.4 & 0.8 & 7.9 & 1.6 & 12.8 & 12.4 & 0.6 & na & 234 & na & na & 5.6 \\
\hline $\begin{array}{l}\text { Hopewell, } \\
\text { N. Mex. }\end{array}$ & $3 / 26 / 1999$ & E & 0.9 & 6.5 & 5.69 & 2.0 & 27.0 & 3.3 & 3.1 & 1.2 & 7.7 & 1.9 & 13.5 & 17.3 & 0.9 & na & 145 & 4.3 & na & 13.6 \\
\hline $\begin{array}{c}\text { Kings Hill, } \\
\text { Mont. }\end{array}$ & 3/10/1999 & E & -3.5 & 4.0 & 5.38 & 4.2 & 5.5 & $<1.7$ & $<1.0$ & 0.8 & 6.8 & 1.1 & 5.0 & 6.7 & 0.9 & na & 183 & 7.2 & na & 14.7 \\
\hline $\begin{array}{c}\text { Lake Irene, } \\
\text { Colo. }\end{array}$ & 4/6/1999 & E & -2.4 & 4.0 & 5.48 & 3.3 & 13.0 & 2.5 & 1.5 & 1.1 & 4.9 & 1.3 & 6.4 & 9.5 & 0.7 & na & 183 & 6.3 & na & 20.9 \\
\hline $\begin{array}{l}\text { Lewis Lake } \\
\text { Divide, } \\
\text { Wyo. }\end{array}$ & 3/3/1999 & E & -3.1 & 6.8 & 5.17 & 6.8 & $<3.1$ & $<1.7$ & 1.9 & 0.4 & 7.4 & 1.5 & 4.3 & 5.1 & 0.4 & na & 332 & 7.0 & na & 20.4 \\
\hline $\begin{array}{c}\text { Lionshead, } \\
\text { Mont. }\end{array}$ & $3 / 6 / 1999$ & E & -4.4 & 5.2 & 5.35 & 4.5 & 3.5 & $<1.7$ & 1.5 & 0.5 & 18.1 & 2.0 & 7.4 & 11.3 & 0.4 & na & 223 & 6.3 & na & 14.9 \\
\hline $\begin{array}{l}\text { Little Brush } \\
\text { Creek, } \\
\text { Utah }\end{array}$ & 3/20/1999 & E & 11.5 & 7.1 & 6.22 & 0.6 & 26.5 & 4.9 & 8.8 & 1.1 & 9.6 & 6.7 & 11.7 & 16.1 & 0.6 & na & 120 & 6.3 & na & 5.7 \\
\hline $\begin{array}{l}\text { Loch Vale For- } \\
\text { est, Colo. }\end{array}$ & 4/13/1999 & E & -2.5 & 6.1 & 5.41 & 3.8 & 17.5 & 3.3 & 2.3 & 1.6 & 6.7 & 1.8 & 10.1 & 14.0 & 1.3 & 90 & 232 & 5.7 & na & 15.2 \\
\hline $\begin{array}{l}\text { Loch Vale } \\
\text { Meadow, } \\
\text { Colo. }\end{array}$ & 4/14/1999 & E & -4.8 & 4.2 & 5.30 & 5.0 & 9.0 & $<1.7$ & 1.4 & 0.5 & 4.4 & 1.0 & 6.5 & 9.1 & $<0.4$ & na & 173 & na & na & 9.9 \\
\hline $\begin{array}{l}\text { Loveland 2, } \\
\text { Colo. }\end{array}$ & 4/26/1999 & E & 2.8 & 5.6 & 5.76 & 1.7 & 20.0 & 3.3 & 4.9 & 1.9 & 5.2 & 3.7 & 8.3 & 13.9 & 1.2 & na & 192 & 4.4 & na & 12.3 \\
\hline $\begin{array}{l}\text { Loveland Pass, } \\
\text { Colo. }\end{array}$ & 4/26/1999 & E & -6.7 & 5.0 & 5.18 & 6.6 & 10.0 & $<1.7$ & 2.3 & 1.0 & 4.1 & 2.3 & 5.3 & 12.3 & 0.5 & na & 238 & 6.0 & na & 9.4 \\
\hline $\begin{array}{c}\text { Lynx Pass, } \\
\text { Colo. }\end{array}$ & 3/10/1999 & E & 4.3 & 4.6 & 5.85 & 1.4 & 19.0 & 2.5 & 2.0 & 0.7 & 3.7 & 1.5 & 7.5 & 13.4 & 0.5 & na & 121 & 5.6 & na & 4.5 \\
\hline $\begin{array}{l}\text { Molas Lake, } \\
\text { Colo. }\end{array}$ & 3/18/1999 & E & 19.9 & 5.8 & 6.44 & 0.4 & 34.4 & 2.5 & 3.5 & 0.7 & 4.2 & 2.3 & 8.0 & 10.0 & $<0.4$ & na & 120 & 3.2 & na & 6.4 \\
\hline $\begin{array}{l}\text { Monarch Pass, } \\
\text { Colo. }\end{array}$ & 3/16/1999 & E & 1.5 & 4.4 & 5.73 & 1.9 & 17.0 & 2.5 & 1.8 & 0.6 & 5.3 & 1.7 & 5.8 & 11.6 & 0.5 & na & 130 & 5.5 & na & 16.8 \\
\hline
\end{tabular}


[E, depth-integrated environmental; ANC, acid neutralization capacity; $\mu \mathrm{eq} / \mathrm{L}$, microequivalents per liter; $\mu \mathrm{S} / \mathrm{cm}$, microsiemens per centimeter; $\mathrm{SC}$, specific conductance at $25^{\circ} \mathrm{C} ; \mathrm{pH}$ values in standard units; $\mathrm{H}$, hydrogen; Ca, calcium; Mg, magnesium; $\mathrm{Na}$, sodium; $\mathrm{K}$, potassium; $\mathrm{NH}_{4}$, ammonium; $\mathrm{Cl}$, chloride; $\mathrm{SO}_{4}$, sulfate; $\mathrm{NO}_{3}$, nitrate; DOC, dissolved organic carbon; mg/L, milligrams per liter; $\mathrm{SWE}$, snow/water equivalent; cm, centimeters; $\delta^{34} \mathrm{~S}$, stable sulfur isotope ratio $\left({ }^{34} \mathrm{~S} /{ }^{22} \mathrm{~S}\right) ; \mathrm{Hg}$, mercury; ng/L, nanograms per liter; na, not analyzed; <, below reporting limits; NP, National Park; \%, percent]

\begin{tabular}{|c|c|c|c|c|c|c|c|c|c|c|c|c|c|c|c|c|c|c|c|c|}
\hline Site name & $\begin{array}{l}\text { Sample } \\
\text { date }\end{array}$ & $\begin{array}{c}\text { Sample } \\
\text { type }\end{array}$ & $\begin{array}{c}\text { ANC } \\
\text { ( } \mu \text { eq/L) }\end{array}$ & $\underset{(\mu \mathrm{S} / \mathrm{cm})}{\mathrm{SC}}$ & $\mathrm{pH}$ & $\begin{array}{c}H \\
(\mu e q / L)\end{array}$ & $\underset{(\mu \mathrm{eq} / \mathrm{L})}{\mathrm{Ca}}$ & $\begin{array}{c}\mathrm{Mg} \\
(\mu \mathrm{eq} / \mathrm{L})\end{array}$ & $\underset{(\mu \mathrm{Na} / \mathrm{L})}{\mathrm{Na}}$ & 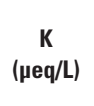 & $\begin{array}{c}\mathrm{NH}_{4} \\
(\mu \mathrm{eq} / \mathrm{L})\end{array}$ & $\underset{\text { (peq/L) }}{C I}$ & $\begin{array}{c}\mathrm{SO}_{4} \\
(\mu \mathrm{eq} / \mathrm{L})\end{array}$ & 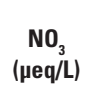 & $\begin{array}{l}\text { DOC } \\
(\mu \mathrm{g} / \mathrm{L})\end{array}$ & $\begin{array}{l}\text { SWE } \\
\text { (cm) }\end{array}$ & $\begin{array}{l}\text { Snow } \\
\text { depth } \\
\text { (cm) }\end{array}$ & $\begin{array}{c}\delta^{34} \mathrm{~S} \\
\text { (per mil) }\end{array}$ & $\underset{(\mathrm{ng} / \mathrm{L})}{\mathrm{Hg}}$ & $\begin{array}{c}\text { Ionic } \\
\text { balance } \\
(\%)\end{array}$ \\
\hline $\begin{array}{l}\text { Monida Pass, } \\
\text { Mont. }\end{array}$ & 3/5/1999 & E & -1.1 & 5.3 & 5.54 & 2.9 & 6.0 & $<1.7$ & 1.7 & 0.5 & 15.6 & 3.1 & 5.7 & 10.9 & 0.4 & na & 117 & na & na & 14.7 \\
\hline $\begin{array}{l}\text { Mount } \\
\text { Belmont, } \\
\text { Mont. }\end{array}$ & 3/6/1999 & E & -5.4 & 3.8 & 5.31 & 4.9 & 5.5 & $<1.7$ & $<1.0$ & 1.0 & 3.3 & 1.1 & 5.5 & 4.6 & 0.8 & na & 125 & na & na & 13.4 \\
\hline $\begin{array}{l}\text { Niwot Snotel, } \\
\text { Colo. }\end{array}$ & $3 / 30 / 1999$ & E & -6.3 & 6.8 & 5.19 & 6.5 & 13.5 & 2.5 & 2.8 & 1.6 & 9.3 & 1.7 & 12.9 & 15.4 & 1.4 & na & 80 & 6.7 & na & 9.2 \\
\hline $\begin{array}{l}\text { Noisy Basin, } \\
\text { Mont. }\end{array}$ & $3 / 11 / 1999$ & E & -7.4 & 3.9 & 5.20 & 6.3 & $<3.1$ & $<1.7$ & $<1.0$ & $<0.4$ & 4.3 & $<1.0$ & 3.4 & 4.9 & 0.4 & na & 371 & na & na & 12.4 \\
\hline $\begin{array}{c}\text { Old Battle, } \\
\text { Wyo. }\end{array}$ & $3 / 25 / 1999$ & E & -9.2 & 5.7 & 5.07 & 8.5 & 8.0 & $<1.7$ & 1.8 & 0.8 & 5.6 & 1.6 & 9.0 & 11.8 & 0.5 & na & 195 & 6.9 & na & 4.8 \\
\hline $\begin{array}{l}\text { Old Faithful } \\
\text { (in road), } \\
\text { Wyo. }\end{array}$ & $3 / 4 / 1999$ & E & -3.7 & 6.9 & 5.22 & 6.0 & 7.5 & $<1.7$ & 4.7 & 0.9 & 15.0 & 2.9 & 12.9 & 9.0 & 2.3 & na & 72 & na & na & 15.8 \\
\hline $\begin{array}{l}\text { Old Faithful } \\
\text { Crew Cor- } \\
\text { rals, Wyo. }\end{array}$ & 3/4/1999 & E & -1.7 & 5.3 & 5.44 & 3.6 & 6.0 & $<1.7$ & 4.3 & $<0.4$ & 8.9 & 1.8 & 7.2 & 7.7 & 0.4 & na & 140 & na & na & 15.9 \\
\hline $\begin{array}{l}\text { Old Faithful } \\
\text { East Lot, } \\
\text { Wyo. }\end{array}$ & $3 / 4 / 1999$ & E & -1.1 & 9.2 & 5.18 & 6.6 & 6.5 & $<1.7$ & 4.7 & $<0.4$ & 10.3 & 2.2 & 6.8 & 8.5 & 0.5 & na & 144 & na & na & 23.2 \\
\hline $\begin{array}{l}\text { Old Faithful } \\
\text { Fire Road, } \\
\text { Wyo. }\end{array}$ & 3/4/1999 & E & -3.6 & 4.5 & 5.31 & 4.9 & 3.5 & $<1.7$ & 2.5 & $<0.4$ & 10.5 & 1.4 & 5.1 & 6.7 & 0.4 & na & 160 & na & na & 23.9 \\
\hline $\begin{array}{l}\text { Phantom Val- } \\
\text { ley, Colo. }\end{array}$ & 4/2/1999 & E & -0.7 & 6.5 & 5.41 & 3.9 & 19.5 & 4.1 & 2.0 & 4.4 & 6.3 & 1.9 & 12.6 & 12.0 & 2.6 & na & 75 & 6.5 & na & 20.6 \\
\hline $\begin{array}{l}\text { Rabbit Ears 1, } \\
\text { Colo. }\end{array}$ & $3 / 30 / 1999$ & E & -11.3 & 6.5 & 4.98 & 10.5 & 7.0 & 2.5 & 1.8 & 1.0 & 7.1 & 1.4 & 9.7 & 11.0 & 0.8 & na & 189 & 7.6 & na & 15.2 \\
\hline $\begin{array}{l}\text { Rabbit Ears 2, } \\
\text { Colo. }\end{array}$ & $3 / 30 / 1999$ & E & -10.5 & 6.3 & 5.02 & 9.5 & 8.0 & $<1.7$ & 1.7 & 1.2 & 6.2 & 1.5 & 10.1 & 12.4 & 1.0 & na & 195 & 7.8 & na & 5.4 \\
\hline $\begin{array}{l}\text { Red Mountain } \\
\text { Pass, Colo. }\end{array}$ & $3 / 18 / 1999$ & E & 21.0 & 6.7 & 6.38 & 0.4 & 38.4 & 4.1 & 4.8 & 4.5 & 5.5 & 2.9 & 9.8 & 13.0 & 1.4 & na & 205 & na & na & 10.5 \\
\hline $\begin{array}{l}\text { Red Mountain, } \\
\text { Mont. }\end{array}$ & $3 / 16 / 1999$ & E & -2.9 & 3.6 & 5.45 & 3.5 & 4.5 & $<1.7$ & 1.1 & 0.6 & 6.7 & $<1.0$ & 3.1 & 5.5 & 0.6 & na & 160 & na & na & 31.2 \\
\hline $\begin{array}{l}\text { Rendezvous } \\
\text { Mountain, } \\
\text { Wyo. }\end{array}$ & $3 / 18 / 1999$ & E & -5.1 & 3.6 & 5.36 & 4.4 & 4.0 & $<1.7$ & 1.7 & $<0.4$ & 4.7 & 1.7 & 4.6 & 3.8 & $<0.4$ & na & 271 & na & na & 18.9 \\
\hline $\begin{array}{l}\text { Ripple Creek } \\
\text { Pass, Colo. }\end{array}$ & 4/9/1999 & E & 75.8 & 11.8 & 6.96 & 0.1 & 77.4 & 5.8 & 5.0 & 4.2 & 5.9 & 2.4 & 12.0 & 12.9 & 1.5 & na & 138 & na & na & -2.4 \\
\hline
\end{tabular}




\section{Table 8. Selected chemical and physical data for Rocky Mountain snowpack samples for 1999.—Continued}

[E, depth-integrated environmental; $\mathrm{ANC}$, acid neutralization capacity; $\mu \mathrm{eq} / \mathrm{L}$, microequivalents per liter; $\mu \mathrm{S} / \mathrm{cm}$, microsiemens per centimeter; $\mathrm{SC}$, specific conductance at $25^{\circ} \mathrm{C}$; $\mathrm{pH}$ values in standard units; $\mathrm{H}$, hydrogen; $\mathrm{Ca}$, calcium; $\mathrm{Mg}$, magnesium; $\mathrm{Na}$, sodium; $\mathrm{K}$, potassium; $\mathrm{NH}_{4}$, ammonium; $\mathrm{Cl}$, chloride; $\mathrm{SO}_{4}$, sulfate; $\mathrm{NO}_{3}$, nitrate; DOC, dissolved organic carbon; mg/L, milligrams per liter; $\mathrm{SWE}$, snow/water equivalent; cm, centimeters; $\delta^{34} \mathrm{~S}$, stable sulfur isotope ratio $\left({ }^{34} \mathrm{~S} /{ }^{22} \mathrm{~S}\right) ; \mathrm{Hg}$, mercury; ng/L, nanograms per liter; na, not analyzed; <, below reporting limits; NP, National Park; \%, percent]

\begin{tabular}{|c|c|c|c|c|c|c|c|c|c|c|c|c|c|c|c|c|c|c|c|c|}
\hline Site name & $\begin{array}{l}\text { Sample } \\
\text { date }\end{array}$ & $\begin{array}{c}\text { Sample } \\
\text { type }\end{array}$ & $\begin{array}{c}\text { ANC } \\
\text { ( } \mu \mathrm{eq} / \mathrm{L})\end{array}$ & $\begin{array}{c}\mathrm{SC} \\
(\mu \mathrm{S} / \mathrm{cm})\end{array}$ & $\mathrm{pH}$ & 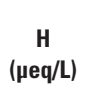 & $\underset{(\mu \mathrm{eq} / \mathrm{L})}{\mathrm{Ca}}$ & $\underset{(\mu \mathrm{eq} / \mathrm{L})}{\mathrm{Mg}}$ & $\underset{\text { (peq/L) }}{\mathrm{Na}}$ & 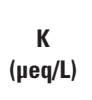 & $\underset{(\mu \mathrm{eq} / \mathrm{L})}{\mathrm{NH}_{4}}$ & 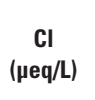 & $\begin{array}{c}\mathrm{SO}_{4} \\
(\mu \mathrm{eq} / \mathrm{L})\end{array}$ & $\begin{array}{c}\mathrm{NO}_{3} \\
(\mu \mathrm{eq} / \mathrm{L})\end{array}$ & $\begin{array}{l}\text { DOC } \\
(\mu \mathrm{g} / \mathrm{L})\end{array}$ & $\begin{array}{l}\text { SWE } \\
(\mathrm{cm})\end{array}$ & $\begin{array}{l}\text { Snow } \\
\text { depth } \\
(\mathrm{cm})\end{array}$ & $\begin{array}{c}\delta^{34} \mathbf{S} \\
\text { (per mil) }\end{array}$ & $\underset{\text { (ng/L) }}{\mathrm{Hg}}$ & $\begin{array}{c}\text { lonic } \\
\text { balance } \\
(\%)\end{array}$ \\
\hline $\begin{array}{l}\text { Slumgullion } \\
\text { Pass, Colo. }\end{array}$ & $3 / 17 / 1999$ & $\mathrm{E}$ & 6.4 & 5.0 & 5.91 & 1.2 & 19.5 & 2.5 & 3.2 & 4.5 & 4.3 & 2.7 & 7.3 & 9.4 & 1.1 & na & 98 & na & na & 15.1 \\
\hline $\begin{array}{c}\text { Snow Bowl, } \\
\text { Mont. }\end{array}$ & $3 / 14 / 1999$ & E & -6.4 & 2.8 & 5.28 & 5.2 & $<3.1$ & $<1.7$ & $<1.0$ & $<0.4$ & 2.3 & $<1.0$ & 2.6 & 2.5 & 0.4 & na & 241 & na & na & 19.0 \\
\hline $\begin{array}{l}\text { South Pass, } \\
\text { Wyo. }\end{array}$ & 3/23/1999 & E & -4.4 & 4.3 & 5.30 & 5.0 & 8.0 & $<1.7$ & 2.6 & 0.4 & 5.2 & 1.4 & 7.6 & 7.7 & 0.5 & na & 103 & na & na & 12.0 \\
\hline $\begin{array}{l}\text { Spring Gulch, } \\
\text { Mont. }\end{array}$ & 3/9/1999 & E & -1.4 & 3.6 & 5.58 & 2.6 & 7.0 & $<1.7$ & $<1.0$ & 1.2 & 7.1 & 1.0 & 4.8 & 6.6 & 0.6 & na & 65 & na & na & 18.2 \\
\hline $\begin{array}{l}\text { Sunlight } \\
\text { Creek, } \\
\text { Mont. }\end{array}$ & 3/8/1999 & E & -3.1 & 3.4 & 5.48 & 3.3 & 5.5 & $<1.7$ & $<1.0$ & 0.5 & 7.1 & 1.0 & 4.2 & 6.1 & 0.6 & na & 120 & na & na & 18.0 \\
\hline $\begin{array}{l}\text { Sunlight Peak, } \\
\text { Colo. }\end{array}$ & 4/6/1999 & E & 29.4 & 5.8 & 6.49 & 0.3 & 36.9 & 2.5 & 2.1 & 1.4 & 4.3 & 1.3 & 6.3 & 8.8 & 0.6 & na & 140 & 4.1 & na & 1.9 \\
\hline $\begin{array}{l}\text { Sylvan Lake } \\
\text { (in road), } \\
\text { Wyo. }\end{array}$ & $3 / 5 / 1999$ & E & -7.7 & 5.7 & 5.06 & 8.7 & $<3.1$ & $<1.7$ & 1.2 & $<0.4$ & 6.2 & $<1.0$ & 4.8 & 6.2 & 0.4 & na & 210 & na & na & 19.1 \\
\hline $\begin{array}{l}\text { Sylvan Lake, } \\
\text { Wyo. }\end{array}$ & 3/5/1999 & E & -5.1 & 3.5 & 5.29 & 5.1 & $<3.1$ & $<1.7$ & $<1.0$ & $<0.4$ & 6.7 & $<1.0$ & 4.1 & 5.2 & 0.5 & na & 233 & na & na & 11.7 \\
\hline $\begin{array}{l}\text { Teton Pass, } \\
\text { Wyo. }\end{array}$ & 3/18/1999 & E & 14.7 & 4.9 & 6.38 & 0.4 & 11.5 & 4.1 & 4.6 & 0.6 & 7.3 & 2.3 & 8.3 & 7.5 & 0.5 & na & 160 & na & na & -7.1 \\
\hline $\begin{array}{l}\text { Togwotee } \\
\text { Pass, Wyo. }\end{array}$ & $3 / 19 / 1999$ & E & -5.3 & 3.2 & 5.36 & 4.4 & $<3.1$ & $<1.7$ & $<1.0$ & 0.7 & 4.2 & 1.0 & 3.5 & 4.9 & 0.5 & na & 175 & na & na & -0.3 \\
\hline $\begin{array}{l}\text { Trappers Lake, } \\
\text { Colo. }\end{array}$ & 4/9/1999 & E & 80.2 & 12.1 & 6.98 & 0.1 & 92.3 & 6.6 & 4.8 & 3.4 & 5.4 & 2.3 & 10.5 & 12.4 & 1.4 & na & 143 & na & na & 3.3 \\
\hline $\begin{array}{c}\text { Trial Lake, } \\
\text { Utah }\end{array}$ & $3 / 21 / 1999$ & E & 15.1 & 5.8 & 6.29 & 0.5 & 24.0 & 3.3 & 10.1 & 0.6 & 7.0 & 5.4 & 9.9 & 9.8 & 0.6 & na & 164 & na & na & 6.1 \\
\hline $\begin{array}{l}\text { Twenty-one } \\
\text { Mile, } \\
\text { Mont. }\end{array}$ & 3/7/1999 & E & -6.5 & 6.2 & 5.20 & 6.3 & 3.5 & $<1.7$ & 4.8 & 2.4 & 10.0 & 2.1 & 5.2 & 8.3 & 0.7 & na & 168 & na & na & 26.7 \\
\hline $\begin{array}{l}\text { Univer- } \\
\text { sity Camp, } \\
\text { Colo. }\end{array}$ & 4/1/1999 & E & -7.6 & 6.3 & 5.14 & 7.2 & 10.0 & $<1.7$ & 1.8 & 1.6 & 6.5 & 1.5 & 9.2 & 13.3 & 1.0 & na & 178 & na & na & 5.9 \\
\hline $\begin{array}{l}\text { W1 (Glacier } \\
\text { NP), Mont. }\end{array}$ & $3 / 22 / 1999$ & E & -3.8 & 3.9 & 5.26 & 5.5 & $<3.1$ & $<1.7$ & 1.6 & 0.6 & 7.7 & 1.3 & 4.5 & 5.1 & 0.8 & 31 & 87 & 6.4 & na & 16.7 \\
\hline $\begin{array}{l}\text { W2 (Glacier } \\
\text { NP), Mont. }\end{array}$ & 3/28/1999 & E & -5.6 & 3.5 & 5.22 & 6.0 & $<3.1$ & $<1.7$ & 1.2 & 0.7 & 5.1 & $<1.0$ & 4.2 & 6.0 & 0.9 & 47 & 126 & na & na & 11.8 \\
\hline
\end{tabular}


[E, depth-integrated environmental; ANC, acid neutralization capacity; $\mu \mathrm{eq} / \mathrm{L}$, microequivalents per liter; $\mathrm{uS} / \mathrm{cm}$, microsiemens per centimeter; $\mathrm{SC}$, specific conductance at $25^{\circ} \mathrm{C} ; \mathrm{pH}$ values in standard units; $\mathrm{H}$, hydrogen; Ca, calcium; Mg, magnesium; $\mathrm{Na}$, sodium; $\mathrm{K}$, potassium; $\mathrm{NH}_{4}$, ammonium; $\mathrm{Cl}$, chloride; $\mathrm{SO}_{4}$, sulfate; $\mathrm{NO}_{3}$, nitrate; DOC, dissolved organic carbon; mg/L, milligrams per liter; $\mathrm{SWE}$, snow/water equivalent; cm, centimeters; $\delta^{34} \mathrm{~S}$, stable sulfur isotope ratio $\left({ }^{34} \mathrm{~S} /{ }^{22} \mathrm{~S}\right) ; \mathrm{Hg}$, mercury; ng/L, nanograms per liter; na, not analyzed; <, below reporting limits; NP, National Park; \%, percent]

\begin{tabular}{|c|c|c|c|c|c|c|c|c|c|c|c|c|c|c|c|c|c|c|c|c|}
\hline Site name & $\begin{array}{l}\text { Sample } \\
\text { date }\end{array}$ & $\begin{array}{c}\text { Sample } \\
\text { type }\end{array}$ & $\begin{array}{c}\text { ANC } \\
(\mu \mathrm{eq} / \mathrm{L})\end{array}$ & $\begin{array}{c}\text { SC } \\
(\mu \mathrm{S} / \mathrm{cm})\end{array}$ & pH & 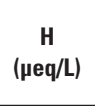 & $\begin{array}{c}\mathrm{Ca} \\
(\mu \mathrm{eq} / \mathrm{L})\end{array}$ & $\begin{array}{c}\mathrm{Mg} \\
(\mu \mathrm{eq} / \mathrm{L})\end{array}$ & $\begin{array}{c}\mathrm{Na} \\
(\mu \mathrm{eq} / \mathrm{L})\end{array}$ & $\begin{array}{c}K \\
(\mu \mathrm{eq} / L)\end{array}$ & $\begin{array}{c}\mathbf{N H}_{4} \\
(\mu \mathrm{eq} / \mathrm{L})\end{array}$ & $\begin{array}{c}\text { CI } \\
(\mu \mathrm{eq} / \mathrm{L})\end{array}$ & $\begin{array}{c}\mathrm{SO}_{4} \\
(\mu \mathrm{eq} / \mathrm{L})\end{array}$ & $\begin{array}{c}\mathrm{NO}_{3} \\
(\mu \mathrm{eq} / \mathrm{L})\end{array}$ & $\begin{array}{l}\text { DOC } \\
(\mu \mathrm{g} / \mathrm{L})\end{array}$ & $\begin{array}{l}\text { SWE } \\
\text { (cm) }\end{array}$ & $\begin{array}{l}\text { Snow } \\
\text { depth } \\
(\mathrm{cm})\end{array}$ & $\begin{array}{c}\delta^{34} \mathbf{S} \\
\text { (per mil) }\end{array}$ & $\begin{array}{c}\mathrm{Hg} \\
\text { (ng/L) }\end{array}$ & $\begin{array}{c}\text { lonic } \\
\text { balance } \\
(\%)\end{array}$ \\
\hline $\begin{array}{l}\text { W3 (Glacier } \\
\text { NP), Mont. }\end{array}$ & 4/1/1999 & E & -6.6 & 3.0 & 5.19 & 6.5 & $<3.1$ & $<1.7$ & 1.4 & $<0.4$ & 2.4 & 1.1 & 3.6 & 2.7 & 0.4 & 129 & 332 & na & na & 16.4 \\
\hline $\begin{array}{l}\text { West Yellow- } \\
\text { stone (in } \\
\text { road), } \\
\text { Mont. }\end{array}$ & 3/6/1999 & E & 9.2 & 8.8 & 5.80 & 1.6 & 17.0 & $<1.7$ & 4.4 & 0.5 & 26.3 & 4.9 & 13.6 & 8.4 & 3.0 & na & 40 & na & na & 15.9 \\
\hline $\begin{array}{l}\text { West Yellow- } \\
\text { stone, } \\
\text { Mont. }\end{array}$ & 3/6/1999 & E & -3.6 & 4.5 & 5.43 & 3.7 & 4.0 & $<1.7$ & 1.8 & 0.6 & 10.9 & 1.8 & 5.0 & 8.5 & 0.8 & na & 126 & na & na & 15.9 \\
\hline $\begin{array}{c}\text { Willow Creek } \\
\text { Pass, Colo. }\end{array}$ & 3/31/1999 & E & 0.7 & 4.6 & 5.65 & 2.2 & 15.5 & 2.5 & 1.6 & 1.6 & 4.3 & 1.3 & 7.4 & 10.8 & 1.1 & na & 108 & 5.9 & na & 15.5 \\
\hline $\begin{array}{l}\text { Willow Creek, } \\
\text { Wyo. }\end{array}$ & $3 / 22 / 1999$ & E & -3.0 & 5.2 & 5.48 & 3.3 & 7.5 & $<1.7$ & 4.3 & 0.6 & 10.3 & 2.5 & 11.3 & 8.3 & 0.5 & na & 237 & 6.9 & na & 8.3 \\
\hline $\begin{array}{l}\text { Wolf Creek } \\
\text { Pass, Colo. }\end{array}$ & 3/17/1999 & E & -0.6 & 5.3 & 5.59 & 2.6 & 17.5 & 2.5 & 2.7 & 3.2 & 5.9 & 1.9 & 11.5 & 10.3 & 1.2 & na & 178 & 4.3 & na & 18.2 \\
\hline
\end{tabular}


Table 9. Selected chemical and physical data for Rocky Mountain snowpack samples for 2000.

[E, depth-integrated environmental; ANC, acid neutralization capacity; $\mu \mathrm{eq} / \mathrm{L}$, microequivalents per liter; $\mu \mathrm{S} / \mathrm{cm}$, microsiemens per centimeter; $\mathrm{SC}$, specific conductance at $25^{\circ} \mathrm{C}$; $\mathrm{pH}$ values in standard units; $\mathrm{H}$, hydrogen; Ca, calcium; $\mathrm{Mg}$, magnesium; $\mathrm{Na}$, sodium; $\mathrm{K}$, potassium; $\mathrm{NH}_{4}$, ammonium; $\mathrm{Cl}$, chloride; $\mathrm{SO}_{4}$, sulfate; $\mathrm{NO}_{3}$, nitrate; DOC, dissolved organic carbon; mg/L, milligrams per liter; $\mathrm{SWE}$, snow/water equivalent; cm, centimeters; $\delta^{34} \mathrm{~S}$, stable sulfur isotope ratio $\left({ }^{34} \mathrm{~S} /{ }^{32} \mathrm{~S}\right) ; \mathrm{Hg}$, mercury; ng/L, nanograms per liter; na, not analyzed; <, below reporting limits; NP, National Park; \%, percent]

\begin{tabular}{|c|c|c|c|c|c|c|c|c|c|c|c|c|c|c|c|c|c|c|c|c|}
\hline Site name & $\begin{array}{l}\text { Sample } \\
\text { date }\end{array}$ & $\begin{array}{l}\text { Sample } \\
\text { type }\end{array}$ & $\begin{array}{c}\text { ANC } \\
(\mu \mathrm{eq} / \mathrm{L})\end{array}$ & $\begin{array}{c}\mathrm{SC} \\
(\mu \mathrm{S} / \mathrm{cm})\end{array}$ & $\mathrm{pH}$ & $\begin{array}{c}\text { H } \\
(\mu \mathrm{eq} / \mathrm{L})\end{array}$ & $\begin{array}{c}\mathrm{Ca} \\
(\mu \mathrm{eq} / \mathrm{L})\end{array}$ & $\underset{(\mu \mathrm{eq} / \mathrm{L})}{\mathrm{Mg}}$ & 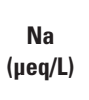 & $\begin{array}{c}K \\
(\mu e q / L)\end{array}$ & $\begin{array}{c}\mathrm{NH}_{4} \\
(\mu \mathrm{eq} / \mathrm{L})\end{array}$ & 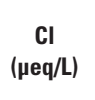 & 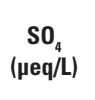 & $\begin{array}{c}\mathrm{NO}_{3} \\
(\mu \mathrm{eq} / \mathrm{L})\end{array}$ & $\begin{array}{c}\text { DOC } \\
(\mu \mathrm{g} / \mathrm{L})\end{array}$ & $\begin{array}{l}\text { SWE } \\
\text { (cm) }\end{array}$ & $\begin{array}{l}\text { Snow } \\
\text { depth } \\
\text { (cm) }\end{array}$ & $\begin{array}{c}\delta^{34} \mathbf{S} \\
\text { (per mil) }\end{array}$ & $\begin{array}{c}\mathrm{Hg} \\
(\mathrm{ng} / \mathrm{L})\end{array}$ & $\begin{array}{c}\text { Ionic } \\
\text { balance } \\
(\%)\end{array}$ \\
\hline $\begin{array}{l}\text { Ajax Moun- } \\
\text { tain, Colo. }\end{array}$ & $3 / 29 / 2000$ & E & -5.4 & 3.7 & 5.15 & 7.1 & 6.5 & 2.5 & $<1.0$ & 2.9 & 4.7 & $<1.0$ & 6.4 & 8.9 & 1.2 & na & 185 & na & na & 21.0 \\
\hline $\begin{array}{l}\text { Apgar Look- } \\
\text { out, Mont. }\end{array}$ & $3 / 6 / 2000$ & E & -5.3 & 4.0 & 5.09 & 8.1 & $<3.1$ & $<1.7$ & $<1.0$ & $<0.4$ & 4.4 & $<1.0$ & 4.9 & 6.1 & 0.4 & 59 & 181 & 7.3 & na & 6.9 \\
\hline $\begin{array}{l}\text { Beaver Moun- } \\
\text { tain, Utah }\end{array}$ & $3 / 23 / 2000$ & E & 10.7 & 4.0 & 5.69 & 2.0 & 13.0 & 2.5 & 7.1 & 1.4 & 6.0 & 3.6 & 7.8 & 3.5 & 0.6 & na & 190 & 8.1 & na & 11.2 \\
\hline $\begin{array}{l}\text { Berthoud Pass, } \\
\text { Colo. }\end{array}$ & $4 / 3 / 2000$ & E & -3.1 & 4.9 & 5.06 & 8.7 & 5.5 & $<1.7$ & 8.3 & 1.2 & 4.5 & 7.8 & 5.3 & 7.8 & 0.7 & na & 193 & 5.2 & na & 14.8 \\
\hline $\begin{array}{l}\text { Big Mountain, } \\
\text { Mont. }\end{array}$ & $3 / 10 / 2000$ & E & -4.3 & 3.3 & 5.19 & 6.5 & $<3.1$ & $<1.7$ & $<1.0$ & $<0.4$ & 2.4 & $<1.0$ & 3.2 & 4.4 & 0.5 & na & 266 & 6.8 & na & 7.2 \\
\hline Big Sky, Mont. & $3 / 15 / 2000$ & E & 3.7 & 2.6 & 5.55 & 2.8 & 5.0 & $<1.7$ & $<1.0$ & 0.8 & 4.0 & $<1.0$ & 2.5 & 4.1 & 0.6 & na & 120 & na & na & 10.1 \\
\hline $\begin{array}{l}\text { Brooklyn Lake, } \\
\text { Wyo. }\end{array}$ & $4 / 14 / 2000$ & E & -6.5 & 3.9 & 4.92 & 12.0 & 5.0 & $<1.7$ & 1.1 & $<0.4$ & 3.9 & $<1.0$ & 5.7 & 7.6 & 0.4 & na & 224 & 6.5 & na & 24.6 \\
\hline Brumley, Colo. & $3 / 24 / 2000$ & E & -3.1 & 4.1 & 5.09 & 8.1 & 5.5 & $<1.7$ & $<1.0$ & 2.0 & 3.9 & $<1.0$ & 5.2 & 7.5 & 0.8 & na & 88 & na & na & 21.1 \\
\hline $\begin{array}{l}\text { Buffalo Pass, } \\
\text { Colo. }\end{array}$ & $4 / 5 / 2000$ & E & -5.6 & 4.7 & 4.93 & 11.7 & 7.0 & $<1.7$ & 1.2 & 0.9 & 5.6 & $<1.0$ & 9.8 & 10.7 & $<0.4$ & na & 339 & 5.3 & na & 12.7 \\
\hline $\begin{array}{l}\text { Burnt Moun- } \\
\text { tain, Colo. }\end{array}$ & $3 / 28 / 2000$ & E & -2.9 & 3.7 & 5.20 & 6.3 & 8.0 & $<1.7$ & 1.1 & 1.6 & 4.7 & $<1.0$ & 6.1 & 9.7 & 1.0 & na & 130 & na & na & 15.8 \\
\hline $\begin{array}{l}\text { Cameron Pass, } \\
\text { Colo. }\end{array}$ & $4 / 4 / 2000$ & E & -3.4 & 4.1 & 5.05 & 8.9 & 5.0 & $<1.7$ & 1.0 & 0.9 & 5.2 & $<1.0$ & 6.4 & 7.7 & 0.7 & na & 201 & na & na & 19.6 \\
\hline Canyon, Wyo. & $3 / 4 / 2000$ & E & -0.6 & 2.9 & 5.36 & 4.4 & 4.0 & $<1.7$ & 1.7 & $<0.4$ & 5.5 & $<1.0$ & 3.4 & 7.7 & na & na & 101 & 5.8 & na & 16.7 \\
\hline $\begin{array}{l}\text { Chief Joseph } \\
\text { Pass, Mont. }\end{array}$ & $3 / 12 / 2000$ & E & -5.3 & 2.5 & 5.26 & 5.5 & $<3.1$ & $<1.7$ & $<1.0$ & $<0.4$ & 2.9 & $<1.0$ & 1.7 & 2.8 & 0.5 & na & 156 & na & na & 30.0 \\
\hline Culebra, Colo. & $2 / 27 / 2000$ & E & -0.7 & 4.6 & 5.47 & 3.4 & 12.2 & 1.8 & 1.6 & 1.4 & 4.3 & $<1.0$ & 5.6 & 9.2 & 0.6 & na & 70 & 5.0 & na & 25.2 \\
\hline $\begin{array}{c}\text { Daisy Pass, } \\
\text { Mont. }\end{array}$ & $3 / 7 / 2000$ & E & -0.8 & 2.4 & 5.48 & 3.3 & 4.0 & $<1.7$ & 1.4 & 0.6 & 3.8 & $<1.0$ & 2.7 & 3.9 & 0.4 & na & 215 & na & na & 33.1 \\
\hline $\begin{array}{l}\text { Deadman Pass, } \\
\text { Colo. }\end{array}$ & $3 / 31 / 2000$ & E & -2.2 & 4.8 & 5.08 & 8.3 & 10.5 & 2.5 & 1.7 & 0.9 & 7.3 & 1.2 & 9.8 & 11.8 & 0.4 & na & 205 & na & na & 15.5 \\
\hline $\begin{array}{c}\text { Divide Peak, } \\
\text { Wyo. }\end{array}$ & $3 / 25 / 2000$ & E & -3.7 & 5.2 & 5.00 & 10.0 & 9.5 & 2.5 & 2.3 & 0.6 & 7.9 & 1.3 & 9.7 & 13.7 & 0.4 & na & 180 & na & na & 13.9 \\
\hline $\begin{array}{c}\text { Dry Lake, } \\
\text { Colo. }\end{array}$ & $3 / 19 / 2000$ & E & -12.6 & 7.1 & 4.86 & 13.8 & 5.5 & $<1.7$ & $<1.0$ & 0.8 & 5.5 & $<1.0$ & 10.1 & 11.9 & 0.6 & na & 160 & 6.0 & na & 7.5 \\
\hline $\begin{array}{l}\text { Dunckley Pass, } \\
\text { Colo. }\end{array}$ & $4 / 6 / 2000$ & E & -0.4 & 3.9 & 5.10 & 7.9 & 8.5 & 2.5 & $<1.0$ & 1.2 & 4.4 & $<1.0$ & 6.0 & 9.7 & 0.4 & na & 158 & 5.9 & na & 21.9 \\
\hline $\begin{array}{l}\text { E2 (Glacier } \\
\text { NP), Mont. }\end{array}$ & $3 / 20 / 2000$ & E & -1.7 & 2.4 & 5.23 & 5.9 & $<3.1$ & $<1.7$ & $<1.0$ & $<0.4$ & 2.7 & $<1.0$ & 2.5 & 2.4 & 0.5 & 38 & 103 & na & na & 27.0 \\
\hline
\end{tabular}


[E, depth-integrated environmental; ANC, acid neutralization capacity; $\mu \mathrm{eq} / \mathrm{L}$, microequivalents per liter; $\mu \mathrm{S} / \mathrm{cm}$, microsiemens per centimeter; $\mathrm{SC}$, specific conductance at $25^{\circ} \mathrm{C} ; \mathrm{pH}$ values in standard units; $\mathrm{H}$, hydrogen; $\mathrm{Ca}$, calcium; $\mathrm{Mg}$, magnesium; $\mathrm{Na}$, sodium; $\mathrm{K}$, potassium; $\mathrm{NH}_{4}$, ammonium; $\mathrm{Cl}$, chloride; $\mathrm{SO}_{4}$, sulfate; $\mathrm{NO}_{3}$, nitrate; DOC, dissolved organic carbon; mg/L, milligrams per liter; $\mathrm{SWE}$, snow/water equivalent; cm, centimeters; $\delta^{34} \mathrm{~S}$, stable sulfur isotope ratio $\left({ }^{34} \mathrm{~S} /{ }^{22} \mathrm{~S}\right) ; \mathrm{Hg}$, mercury; ng/L, nanograms per liter; na, not analyzed; <, below reporting limits; NP, National Park; \%, percent]

\begin{tabular}{|c|c|c|c|c|c|c|c|c|c|c|c|c|c|c|c|c|c|c|c|c|}
\hline Site name & $\begin{array}{l}\text { Sample } \\
\text { date }\end{array}$ & $\begin{array}{l}\text { Sample } \\
\text { type }\end{array}$ & 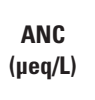 & $\begin{array}{c}\mathrm{SC} \\
(\mu \mathrm{S} / \mathrm{cm})\end{array}$ & $\mathrm{pH}$ & $\begin{array}{c}H \\
(\mu \mathrm{eq} / \mathrm{L})\end{array}$ & $\begin{array}{c}\text { Ca } \\
(\mu \mathrm{eq} / \mathrm{L})\end{array}$ & $\underset{(\mu \mathrm{eq} / \mathrm{L})}{\mathrm{Mg}}$ & $\begin{array}{c}\mathrm{Na} \\
(\mu \mathrm{eq} / \mathrm{L})\end{array}$ & $\begin{array}{c}K \\
(\mu e q / L)\end{array}$ & $\begin{array}{c}\mathrm{NH}_{4} \\
(\mu \mathrm{eq} / \mathrm{L})\end{array}$ & 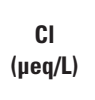 & 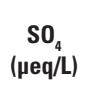 & $\begin{array}{c}\mathrm{NO}_{3} \\
(\mu \mathrm{eq} / \mathrm{L})\end{array}$ & $\begin{array}{l}\text { DOC } \\
(\mu \mathrm{g} / \mathrm{L})\end{array}$ & $\begin{array}{l}\text { SWE } \\
\text { (cm) }\end{array}$ & $\begin{array}{l}\text { Snow } \\
\text { depth } \\
\text { (cm) }\end{array}$ & $\begin{array}{c}\delta^{34} \mathrm{~S} \\
\text { (per mil) }\end{array}$ & $\begin{array}{c}\mathrm{Hg} \\
(\mathbf{n g} / \mathrm{L})\end{array}$ & $\begin{array}{c}\text { lonic } \\
\text { balance } \\
(\%)\end{array}$ \\
\hline $\begin{array}{l}\text { E3 (Glacier } \\
\text { NP), Mont. }\end{array}$ & $3 / 22 / 2000$ & E & -5.6 & 2.2 & 5.17 & 6.8 & $<3.1$ & $<1.7$ & $<1.0$ & 0.4 & 3.8 & $<1.0$ & 2.4 & 3.9 & 0.6 & 49 & 130 & na & na & 27.1 \\
\hline $\begin{array}{l}\text { E4 (Glacier } \\
\text { NP), Mont. }\end{array}$ & $3 / 21 / 2000$ & E & -2.3 & 3.2 & 5.26 & 5.5 & $<3.1$ & $<1.7$ & 5.9 & 1.2 & 3.7 & $<1.0$ & 5.0 & 6.2 & 1.4 & 73 & 190 & na & na & 18.5 \\
\hline $\begin{array}{l}\text { E5 (Glacier } \\
\text { NP), Mont. }\end{array}$ & $3 / 21 / 2000$ & E & -7.3 & 3.9 & 5.02 & 9.5 & $<3.1$ & $<1.7$ & $<1.0$ & 0.4 & 2.4 & $<1.0$ & 3.9 & 3.4 & 0.8 & 93 & 245 & na & na & 26.1 \\
\hline $\begin{array}{l}\text { E6 (Glacier } \\
\text { NP), Mont. }\end{array}$ & $3 / 21 / 2000$ & E & -8.5 & 3.7 & 5.03 & 9.3 & $<3.1$ & $<1.7$ & $<1.0$ & 0.7 & 2.7 & $<1.0$ & 4.4 & 4.2 & 0.7 & 80 & 225 & na & na & 19.8 \\
\hline $\begin{array}{l}\text { E7 (Glacier } \\
\text { NP), Mont. }\end{array}$ & $3 / 22 / 2000$ & E & -6.5 & 3.0 & 5.07 & 8.5 & $<3.1$ & $<1.7$ & 1.1 & 1.8 & 3.8 & $<1.0$ & 4.5 & 4.8 & 1.6 & 76 & 220 & na & na & 23.8 \\
\hline $\begin{array}{c}\text { Elk River, } \\
\text { Colo. }\end{array}$ & $3 / 19 / 2000$ & E & -5.4 & 4.6 & 5.10 & 7.9 & 7.5 & $<1.7$ & 1.5 & 0.5 & 3.9 & $<1.0$ & 6.9 & 11.4 & 0.6 & na & 145 & na & na & 7.8 \\
\hline $\begin{array}{c}\text { Elkhart Park, } \\
\text { Wyo. }\end{array}$ & $3 / 8 / 2000$ & E & -3.5 & 3.2 & 5.28 & 5.2 & 4.0 & $<1.7$ & 1.8 & 0.4 & 4.3 & $<1.0$ & 4.4 & 6.3 & 0.4 & na & 127 & na & na & 19.3 \\
\hline $\begin{array}{l}\text { Four Mile } \\
\text { Meadow, } \\
\text { Wyo. }\end{array}$ & $3 / 7 / 2000$ & E & -0.8 & 2.9 & 5.46 & 3.5 & 5.5 & $<1.7$ & 2.3 & 0.6 & 3.9 & $<1.0$ & 3.4 & 5.9 & 0.6 & na & 94 & na & na & 26.4 \\
\hline $\begin{array}{l}\text { Fremont Pass, } \\
\text { Colo. }\end{array}$ & $4 / 17 / 2000$ & E & -2.6 & 3.8 & 5.14 & 7.2 & 8.0 & $<1.7$ & 1.3 & 0.9 & 3.9 & 1.0 & 5.6 & 8.1 & $<0.4$ & na & 140 & 4.6 & na & 18.8 \\
\hline $\begin{array}{l}\text { Garnet Can- } \\
\text { yon, Wyo. }\end{array}$ & $3 / 22 / 2000$ & E & 6.0 & 3.3 & 5.73 & 1.9 & 8.0 & 2.5 & 3.4 & 0.8 & 5.8 & $<1.0$ & 5.8 & 5.7 & 0.7 & na & 175 & 6.6 & na & 12.2 \\
\hline $\begin{array}{l}\text { Grand Mesa, } \\
\text { Colo. }\end{array}$ & $3 / 22 / 2000$ & E & -1.6 & 5.1 & 5.18 & 6.6 & 11.0 & 2.5 & 1.6 & 1.4 & 6.7 & 1.2 & 8.8 & 10.5 & 0.7 & na & 160 & na & na & 18.3 \\
\hline $\begin{array}{l}\text { Granite Park, } \\
\text { Mont. }\end{array}$ & $3 / 22 / 2000$ & E & -8.3 & 2.8 & 5.03 & 9.3 & $<3.1$ & $<1.7$ & $<1.0$ & $<0.4$ & 4.1 & $<1.0$ & 4.6 & 4.9 & na & 105 & 275 & na & na & 17.5 \\
\hline $\begin{array}{l}\text { Granite Pass, } \\
\text { Mont. }\end{array}$ & $3 / 13 / 2000$ & E & -1.4 & 2.2 & 5.24 & 5.8 & $<3.1$ & $<1.7$ & $<1.0$ & $<0.4$ & 1.6 & $<1.0$ & 2.1 & 2.0 & 0.8 & na & 211 & 6.8 & na & 28.3 \\
\hline $\begin{array}{l}\text { Gypsum Creek, } \\
\text { Wyo. }\end{array}$ & $3 / 8 / 2000$ & E & -5.1 & 3.1 & 5.28 & 5.2 & 4.0 & $<1.7$ & 3.4 & 1.6 & 3.4 & $<1.0$ & 4.0 & 5.5 & $<0.4$ & na & 133 & na & na & 30.3 \\
\hline $\begin{array}{l}\text { Hopewell, } \\
\text { N. Mex. }\end{array}$ & $3 / 12 / 2000$ & E & 1.4 & 5.0 & 5.42 & 3.8 & 22.0 & 3.3 & 2.6 & 1.1 & 4.3 & 1.0 & 8.6 & 12.6 & 0.8 & na & 102 & 5.3 & na & 22.3 \\
\hline $\begin{array}{l}\text { Independence } \\
\text { Pass , Colo. }\end{array}$ & $4 / 10 / 2000$ & E & -1.5 & 3.8 & 5.14 & 7.2 & 9.5 & 2.5 & 1.3 & 1.6 & 3.7 & 1.3 & 5.2 & 8.5 & 0.9 & na & 140 & na & na & 26.5 \\
\hline $\begin{array}{l}\text { Independence } \\
\text { Pass (in } \\
\text { road), Colo. }\end{array}$ & $4 / 10 / 2000$ & E & -6.6 & 4.1 & 4.90 & 12.6 & 4.0 & $<1.7$ & $<1.0$ & $<0.4$ & 3.4 & $<1.0$ & 4.2 & 7.4 & 0.5 & na & 130 & na & na & 26.6 \\
\hline
\end{tabular}




\section{Table 9. Selected chemical and physical data for Rocky Mountain snowpack samples for 2000.—Continued}

[E, depth-integrated environmental; ANC, acid neutralization capacity; $\mu \mathrm{eq} / \mathrm{L}$, microequivalents per liter; $\mu \mathrm{S} / \mathrm{cm}$, microsiemens per centimeter; $\mathrm{SC}$, specific conductance at $25^{\circ} \mathrm{C} ; \mathrm{pH}$ values in standard units; $\mathrm{H}$, hydrogen; $\mathrm{Ca}$, calcium; $\mathrm{Mg}$, magnesium; $\mathrm{Na}$, sodium; $\mathrm{K}$, potassium; $\mathrm{NH}_{4}$, ammonium; $\mathrm{Cl}$, chloride; $\mathrm{SO}_{4}$, sulfate; $\mathrm{NO}_{3}$, nitrate; $\mathrm{DOC}$, dissolved organic carbon; mg/L, milligrams per liter; $\mathrm{SWE}$, snow/water equivalent; cm, centimeters; $\delta^{34} \mathrm{~S}$, stable sulfur isotope ratio $\left({ }^{34} \mathrm{~S} /{ }^{22} \mathrm{~S}\right) ; \mathrm{Hg}$, mercury; ng/L, nanograms per liter; na, not analyzed; <, below reporting limits; NP, National Park; \%, percent]

\begin{tabular}{|c|c|c|c|c|c|c|c|c|c|c|c|c|c|c|c|c|c|c|c|c|}
\hline Site name & $\begin{array}{l}\text { Sample } \\
\text { date }\end{array}$ & $\begin{array}{l}\text { Sample } \\
\text { type }\end{array}$ & 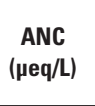 & $\begin{array}{c}\mathrm{SC} \\
(\mu \mathrm{S} / \mathrm{cm})\end{array}$ & $\mathrm{pH}$ & 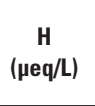 & $\underset{(\mu \mathrm{eq} / \mathrm{L})}{\mathrm{Ca}}$ & $\begin{array}{c}M g \\
(\mu e q / L)\end{array}$ & 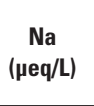 & $\begin{array}{c}K \\
(\mu e q / L)\end{array}$ & 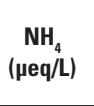 & 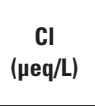 & 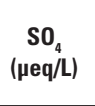 & $\begin{array}{c}\mathrm{NO}_{3} \\
(\mu \mathrm{eq} / \mathrm{L})\end{array}$ & $\begin{array}{l}\text { DOC } \\
(\mu \mathrm{g} / \mathrm{L})\end{array}$ & $\begin{array}{l}\text { SWE } \\
\text { (cm) }\end{array}$ & $\begin{array}{l}\text { Snow } \\
\text { depth } \\
(\mathrm{cm})\end{array}$ & $\begin{array}{c}\delta^{34} \mathrm{~S} \\
\text { (per mil) }\end{array}$ & $\begin{array}{c}\mathrm{Hg} \\
\text { (ng/L) }\end{array}$ & $\begin{array}{c}\text { lonic } \\
\text { balance } \\
(\%)\end{array}$ \\
\hline $\begin{array}{l}\text { Kings Hill, } \\
\text { Mont. }\end{array}$ & $3 / 8 / 2000$ & E & -2.6 & 3.0 & 5.36 & 4.4 & 3.5 & $<1.7$ & $<1.0$ & 0.6 & 6.0 & $<1.0$ & 5.1 & 6.2 & 0.7 & na & 105 & 8.5 & na & 12.7 \\
\hline $\begin{array}{c}\text { Lake Irene, } \\
\text { Colo. }\end{array}$ & $3 / 27 / 2000$ & E & -6.3 & 4.4 & 5.01 & 9.8 & 4.5 & $<1.7$ & $<1.0$ & 0.6 & 4.1 & $<1.0$ & 5.5 & 8.6 & 0.6 & na & 188 & 5.8 & na & 14.7 \\
\hline $\begin{array}{c}\text { Lewis Lake } \\
\text { Divide, } \\
\text { Wyo. }\end{array}$ & $3 / 3 / 2000$ & E & 4.9 & 3.0 & 5.73 & 1.9 & 6.0 & $<1.7$ & 3.6 & 0.4 & 8.2 & $<1.0$ & 5.1 & 8.5 & na & na & 227 & 6.9 & na & 4.2 \\
\hline $\begin{array}{l}\text { Lionshead, } \\
\text { Mont. }\end{array}$ & $3 / 6 / 2000$ & E & 0.9 & 4.6 & 5.37 & 4.3 & 6.5 & 2.5 & 3.7 & 6.0 & 14.4 & 1.7 & 9.7 & 13.9 & 1.1 & na & 136 & 5.6 & na & 17.6 \\
\hline $\begin{array}{l}\text { Little Brush } \\
\text { Creek, Utah }\end{array}$ & $3 / 23 / 2000$ & E & 4.7 & 4.2 & 5.42 & 3.8 & 12.5 & 4.1 & 4.2 & 0.6 & 8.2 & 4.0 & 7.3 & 9.9 & 0.6 & na & 80 & na & na & 12.7 \\
\hline $\begin{array}{l}\text { Lizard Head } \\
\text { Pass, Colo. }\end{array}$ & $2 / 26 / 2000$ & E & 1.1 & 4.7 & 5.45 & 3.5 & 9.5 & $<1.7$ & $<1.0$ & 0.6 & 2.3 & $<1.0$ & 4.2 & 11.1 & 0.4 & na & 128 & 4.8 & na & -1.3 \\
\hline $\begin{array}{l}\text { Loch Vale For- } \\
\text { est, Colo. }\end{array}$ & $4 / 11 / 2000$ & E & -3.4 & 4.9 & 5.03 & 9.3 & 8.0 & $<1.7$ & 1.5 & 0.6 & 6.7 & $<1.0$ & 8.1 & 10.9 & 0.4 & 92 & 230 & 5.1 & na & 16.0 \\
\hline $\begin{array}{l}\text { Loch Vale } \\
\text { Meadow, } \\
\text { Colo. }\end{array}$ & $4 / 11 / 2000$ & E & -5.4 & 4.4 & 4.99 & 10.2 & 5.0 & $<1.7$ & 1.2 & $<0.4$ & 4.6 & $<1.0$ & 6.9 & 6.4 & $<0.4$ & 71 & 173 & 4.9 & na & 22.6 \\
\hline $\begin{array}{l}\text { Loveland 2, } \\
\text { Colo. }\end{array}$ & $4 / 21 / 2000$ & E & -1.8 & 3.8 & 5.19 & 6.5 & 9.0 & 2.5 & 2.9 & 2.3 & 4.2 & 3.1 & 5.7 & 6.5 & 0.9 & na & 188 & na & na & 28.4 \\
\hline $\begin{array}{l}\text { Loveland 3, } \\
\text { Colo. }\end{array}$ & $4 / 21 / 2000$ & E & 39.9 & 8.9 & 6.54 & 0.3 & 40.9 & 11.5 & 22.7 & 4.2 & 3.2 & 21.6 & 10.0 & 4.8 & 2.7 & na & 186 & na & na & 4.1 \\
\hline $\begin{array}{l}\text { Loveland Pass, } \\
\text { Colo. }\end{array}$ & $4 / 19 / 2000$ & E & -2.2 & 3.1 & 5.15 & 7.1 & 7.0 & 2.5 & 2.2 & 0.5 & 2.9 & 2.1 & 4.9 & 4.6 & $<0.4$ & na & 188 & 4.0 & na & 31.3 \\
\hline $\begin{array}{l}\text { Lynx Pass, } \\
\text { Colo. }\end{array}$ & $3 / 18 / 2000$ & E & -7.9 & 5.4 & 5.03 & 9.3 & 6.5 & $<1.7$ & 1.1 & 0.6 & 3.3 & $<1.0$ & 6.4 & 10.5 & 0.5 & na & 143 & 6.5 & na & 10.5 \\
\hline $\begin{array}{l}\text { Molas Lake, } \\
\text { Colo. }\end{array}$ & $3 / 26 / 2000$ & E & -3.7 & 3.6 & 5.20 & 6.3 & 7.5 & $<1.7$ & 1.2 & 0.6 & 2.9 & $<1.0$ & 4.0 & 8.0 & 0.6 & na & 138 & 5.1 & na & 20.9 \\
\hline $\begin{array}{l}\text { Monarch Pass, } \\
\text { Colo. }\end{array}$ & $3 / 24 / 2000$ & E & 9.4 & 4.3 & 5.88 & 1.3 & 19.0 & 4.1 & 2.7 & 0.8 & 5.4 & 1.2 & 8.1 & 10.2 & 0.9 & na & 126 & 5.3 & na & 7.0 \\
\hline $\begin{array}{l}\text { Monida Pass, } \\
\text { Mont. }\end{array}$ & $3 / 2 / 2000$ & E & -2.7 & 5.2 & 5.20 & 6.3 & 4.5 & $<1.7$ & 2.5 & 0.8 & 19.1 & 2.1 & 7.5 & 15.3 & na & na & 44 & na & na & 14.2 \\
\hline $\begin{array}{l}\text { Mount } \\
\text { Belmont, } \\
\text { Mont. }\end{array}$ & $3 / 3 / 2000$ & E & -3.9 & 3.3 & 5.01 & 9.8 & $<3.1$ & $<1.7$ & $<1.0$ & 0.8 & 3.9 & $<1.0$ & 4.1 & 5.2 & 0.7 & na & 62 & na & na & 21.3 \\
\hline $\begin{array}{l}\text { Niwot Snotel, } \\
\text { Colo. }\end{array}$ & $3 / 30 / 2000$ & E & -3.1 & 4.6 & 5.04 & 9.1 & 6.5 & $<1.7$ & 1.3 & 1.0 & 8.4 & $<1.0$ & 8.9 & 9.3 & 1.1 & na & 106 & 6.2 & na & 18.1 \\
\hline
\end{tabular}


[E, depth-integrated environmental; ANC, acid neutralization capacity; $\mu \mathrm{eq} / \mathrm{L}$, microequivalents per liter; $\mu \mathrm{S} / \mathrm{cm}$, microsiemens per centimeter; $\mathrm{SC}$, specific conductance at $25^{\circ} \mathrm{C} ; \mathrm{pH}$ values in standard units; $\mathrm{H}$, hydrogen; Ca, calcium; Mg, magnesium; $\mathrm{Na}$, sodium; $\mathrm{K}$, potassium; $\mathrm{NH}_{4}$, ammonium; $\mathrm{Cl}$, chloride; $\mathrm{SO}_{4}$, sulfate; $\mathrm{NO}_{3}$, nitrate; DOC, dissolved organic carbon; mg/L, milligrams per liter; $\mathrm{SWE}$, snow/water equivalent; cm, centimeters; $\delta^{34} \mathrm{~S}$, stable sulfur isotope ratio $\left({ }^{34} \mathrm{~S} /{ }^{22} \mathrm{~S}\right) ; \mathrm{Hg}$, mercury; ng/L, nanograms per liter; na, not analyzed; <, below reporting limits; NP, National Park; \%, percent]

\begin{tabular}{|c|c|c|c|c|c|c|c|c|c|c|c|c|c|c|c|c|c|c|c|c|}
\hline Site name & $\begin{array}{l}\text { Sample } \\
\text { date }\end{array}$ & $\begin{array}{l}\text { Sample } \\
\text { type }\end{array}$ & $\begin{array}{c}\text { ANC } \\
\text { ( } \mu \mathrm{eq} / \mathrm{L})\end{array}$ & $\begin{array}{c}\mathrm{SC} \\
(\mu \mathrm{S} / \mathrm{cm})\end{array}$ & $\mathrm{pH}$ & $\begin{array}{c}H \\
(\mu e q / L)\end{array}$ & $\begin{array}{c}\mathrm{Ca} \\
(\mu \mathrm{eq} / \mathrm{L})\end{array}$ & $\underset{(\mu \mathrm{eq} / \mathrm{L})}{\mathrm{Mg}}$ & $\begin{array}{c}\mathrm{Na} \\
(\mu \mathrm{eq} / \mathrm{L})\end{array}$ & $\begin{array}{c}K \\
\text { (peq/L) }\end{array}$ & $\begin{array}{c}\mathrm{NH}_{4} \\
(\mu \mathrm{eq} / \mathrm{L})\end{array}$ & 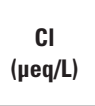 & 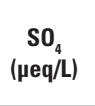 & $\begin{array}{c}\mathrm{NO}_{3} \\
(\mu \mathrm{eq} / \mathrm{L})\end{array}$ & $\begin{array}{l}\text { DOC } \\
(\mu \mathrm{g} / \mathrm{L})\end{array}$ & $\begin{array}{l}\text { SWE } \\
\text { (cm) }\end{array}$ & $\begin{array}{l}\text { Snow } \\
\text { depth } \\
\text { (cm) }\end{array}$ & $\begin{array}{c}\delta^{34} \mathbf{S} \\
\text { (per mil) }\end{array}$ & $\underset{\text { (ng/L) }}{\mathrm{Hg}}$ & $\begin{array}{c}\text { lonic } \\
\text { balance } \\
(\%)\end{array}$ \\
\hline $\begin{array}{l}\text { Noisy Basin, } \\
\text { Mont. }\end{array}$ & 3/9/2000 & E & -6.1 & 3.5 & 5.12 & 7.6 & $<3.1$ & $<1.7$ & $<1.0$ & $<0.4$ & 4.1 & $<1.0$ & 3.6 & 5.4 & 0.5 & na & 302 & na & na & 13.4 \\
\hline $\begin{array}{c}\text { Old Battle, } \\
\text { Wyo. }\end{array}$ & $3 / 25 / 2000$ & E & -7.5 & 5.1 & 4.93 & 11.7 & 5.0 & $<1.7$ & 1.2 & 0.5 & 5.0 & $<1.0$ & 7.5 & 9.1 & 0.4 & na & 240 & 5.7 & na & 17.1 \\
\hline $\begin{array}{l}\text { Old Faithful } \\
\text { Fire Road, } \\
\text { Wyo. }\end{array}$ & $3 / 3 / 2000$ & E & 11.0 & 4.3 & 6.07 & 0.9 & 14.0 & 2.5 & 7.3 & 0.8 & 9.8 & 1.9 & 7.1 & 7.6 & na & na & 96 & na & na & 12.1 \\
\hline $\begin{array}{l}\text { Old Faithful } \\
\text { (in road), } \\
\text { Wyo. }\end{array}$ & $3 / 3 / 2000$ & E & 6.9 & 5.2 & 5.61 & 2.5 & 15.0 & 3.3 & 3.0 & 2.5 & 14.4 & 2.9 & 10.7 & 9.6 & na & na & 45 & na & na & 14.8 \\
\hline $\begin{array}{l}\text { Phantom Val- } \\
\text { ley, Colo. }\end{array}$ & $3 / 19 / 2000$ & E & -7.7 & 4.8 & 5.00 & 10.0 & 5.5 & $<1.7$ & $<1.0$ & 0.7 & 4.2 & $<1.0$ & 6.3 & 9.7 & 0.8 & na & 103 & na & na & 11.9 \\
\hline $\begin{array}{l}\text { Rabbit Ears 1, } \\
\text { Colo. }\end{array}$ & $4 / 3 / 2000$ & E & -7.8 & 5.6 & 4.99 & 10.2 & 6.0 & $<1.7$ & 1.2 & 0.6 & 5.4 & $<1.0$ & 8.5 & 10.4 & $<0.4$ & na & 258 & 6.4 & na & 10.5 \\
\hline $\begin{array}{l}\text { Rabbit Ears 2, } \\
\text { Colo. }\end{array}$ & $4 / 3 / 2000$ & E & -9.6 & 5.6 & 4.88 & 13.2 & 6.0 & $<1.7$ & 1.8 & 0.8 & 5.6 & $<1.0$ & 9.4 & 11.4 & 0.5 & na & 278 & 4.3 & na & 13.6 \\
\hline $\begin{array}{l}\text { Red Mountain, } \\
\text { Mont. }\end{array}$ & $3 / 14 / 2000$ & E & -0.7 & 3.3 & 5.27 & 5.4 & $<3.1$ & $<1.7$ & $<1.0$ & $<0.4$ & 4.0 & $<1.0$ & 3.0 & 4.2 & 0.8 & na & 118 & na & na & 12.6 \\
\hline $\begin{array}{l}\text { Red Mountain } \\
\text { Pass, Colo. }\end{array}$ & $3 / 26 / 2000$ & E & -3.3 & 3.6 & 5.20 & 6.3 & 8.5 & $<1.7$ & 1.3 & 0.8 & 2.4 & $<1.0$ & 4.6 & 8.3 & 0.7 & na & 170 & na & na & 20.0 \\
\hline $\begin{array}{l}\text { Rendezvous } \\
\text { Mountain, } \\
\text { Wyo. }\end{array}$ & $3 / 16 / 2000$ & E & 1.1 & 2.9 & 5.45 & 3.5 & 5.0 & $<1.7$ & 1.8 & $<0.4$ & 4.8 & $<1.0$ & 4.3 & 4.4 & 0.4 & na & 212 & na & na & 21.1 \\
\hline $\begin{array}{l}\text { Slumgullion } \\
\text { Pass, Colo. }\end{array}$ & $3 / 25 / 2000$ & E & -0.3 & 3.5 & 5.28 & 5.2 & 10.0 & $<1.7$ & 1.9 & 2.4 & 2.9 & 1.3 & 5.1 & 6.8 & 1.0 & na & 102 & na & na & 25.9 \\
\hline $\begin{array}{c}\text { Snow Bowl, } \\
\text { Mont. }\end{array}$ & $3 / 11 / 2000$ & E & -4.6 & 2.8 & 5.23 & 5.9 & $<3.1$ & $<1.7$ & $<1.0$ & $<0.4$ & 2.0 & $<1.0$ & 3.0 & 3.4 & 0.5 & na & 145 & na & na & 11.4 \\
\hline $\begin{array}{c}\text { South Pass, } \\
\text { Wyo. }\end{array}$ & $3 / 24 / 2000$ & E & -3.2 & 3.7 & 5.12 & 7.6 & 6.0 & $<1.7$ & $<1.0$ & $<0.4$ & 3.9 & 1.0 & 7.5 & 6.0 & 0.4 & na & 120 & na & na & 9.1 \\
\hline $\begin{array}{l}\text { Spring Gulch, } \\
\text { Mont. }\end{array}$ & $3 / 7 / 2000$ & E & 4.6 & 3.4 & 5.41 & 3.9 & 9.0 & 3.3 & $<1.0$ & 8.2 & 7.0 & $<1.0$ & 6.8 & 7.0 & 2.1 & na & 52 & na & na & 26.2 \\
\hline $\begin{array}{l}\text { Sunlight Creek, } \\
\text { Mont. }\end{array}$ & $3 / 6 / 2000$ & E & 0.9 & 3.1 & 5.46 & 3.5 & 5.0 & $<1.7$ & $<1.0$ & 1.5 & 5.4 & $<1.0$ & 3.7 & 6.7 & na & na & 73 & na & na & 15.3 \\
\hline $\begin{array}{l}\text { Sunlight Peak, } \\
\text { Colo. }\end{array}$ & $3 / 27 / 2000$ & E & -4.0 & 4.0 & 4.99 & 10.2 & 7.5 & $<1.7$ & 1.4 & 0.5 & 4.8 & $<1.0$ & 6.2 & 8.3 & 0.4 & na & 160 & 6.2 & na & 25.4 \\
\hline $\begin{array}{c}\text { Sylvan Lake, } \\
\text { Wyo. }\end{array}$ & $3 / 4 / 2000$ & E & -0.3 & 2.7 & 5.42 & 3.8 & 4.0 & $<1.7$ & 1.6 & 0.5 & 5.8 & $<1.0$ & 4.0 & 7.5 & na & na & 150 & na & na & 15.2 \\
\hline
\end{tabular}




\section{Table 9. Selected chemical and physical data for Rocky Mountain snowpack samples for 2000.—Continued}

[E, depth-integrated environmental; ANC, acid neutralization capacity; $\mu \mathrm{eq} / \mathrm{L}$, microequivalents per liter; $\mu \mathrm{S} / \mathrm{cm}$, microsiemens per centimeter; $\mathrm{SC}$, specific conductance at $25^{\circ} \mathrm{C} ; \mathrm{pH}$ values in standard units; $\mathrm{H}$, hydrogen; $\mathrm{Ca}$, calcium; $\mathrm{Mg}$, magnesium; $\mathrm{Na}$, sodium; $\mathrm{K}$, potassium; $\mathrm{NH}_{4}$, ammonium; $\mathrm{Cl}$, chloride; $\mathrm{SO}_{4}$, sulfate; $\mathrm{NO}_{3}$, nitrate; DOC, dissolved organic carbon; mg/L, milligrams per liter; $\mathrm{SWE}$, snow/water equivalent; cm, centimeters; $\delta^{34} \mathrm{~S}$, stable sulfur isotope ratio $\left({ }^{34} \mathrm{~S} /{ }^{22} \mathrm{~S}\right) ; \mathrm{Hg}$, mercury; ng/L, nanograms per liter; na, not analyzed; <, below reporting limits; NP, National Park; \%, percent]

\begin{tabular}{|c|c|c|c|c|c|c|c|c|c|c|c|c|c|c|c|c|c|c|c|c|}
\hline Site name & $\begin{array}{l}\text { Sample } \\
\text { date }\end{array}$ & $\begin{array}{l}\text { Sample } \\
\text { type }\end{array}$ & 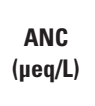 & $\begin{array}{c}\text { SC } \\
(\mu \mathrm{S} / \mathrm{cm})\end{array}$ & $\mathrm{pH}$ & $\begin{array}{c}\text { H } \\
(\mu \mathrm{eq} / \mathrm{L})\end{array}$ & $\begin{array}{c}\mathrm{Ca} \\
(\mu \mathrm{eq} / \mathrm{L})\end{array}$ & $\underset{(\mu \mathrm{g} q / \mathrm{L})}{\mathrm{Mg}}$ & $\begin{array}{c}\mathrm{Na} \\
(\mu \mathrm{eq} / \mathrm{L})\end{array}$ & $\begin{array}{c}K \\
(\mu e q / L)\end{array}$ & $\begin{array}{c}\mathrm{NH}_{4} \\
(\mu \mathrm{eq} / \mathrm{L})\end{array}$ & 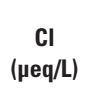 & 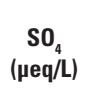 & $\begin{array}{c}\mathrm{NO}_{3} \\
(\mu \mathrm{eq} / \mathrm{L})\end{array}$ & $\begin{array}{l}\text { DOC } \\
(\mu \mathrm{g} / \mathrm{L})\end{array}$ & $\begin{array}{l}\text { SWE } \\
\text { (cm) }\end{array}$ & $\begin{array}{l}\text { Snow } \\
\text { depth } \\
(\mathrm{cm})\end{array}$ & $\begin{array}{c}\delta^{34} \mathrm{~S} \\
\text { (per mil) }\end{array}$ & $\begin{array}{c}\mathrm{Hg} \\
(\mathbf{n g} / \mathrm{L})\end{array}$ & $\begin{array}{c}\text { lonic } \\
\text { balance } \\
(\%)\end{array}$ \\
\hline $\begin{array}{c}\text { Sylvan Lake } \\
\text { (in road), } \\
\text { Wyo. }\end{array}$ & $3 / 4 / 2000$ & E & -1.8 & 2.9 & 5.34 & 4.6 & 3.5 & $<1.7$ & 1.2 & $<0.4$ & 6.2 & $<1.0$ & 5.1 & 6.1 & na & na & 125 & na & na & 16.5 \\
\hline $\begin{array}{l}\text { Taos Ski Val- } \\
\text { ley, N. Mex. }\end{array}$ & $3 / 11 / 2000$ & E & -1.0 & 4.6 & 5.40 & 4.0 & 15.5 & 2.5 & 1.4 & 1.0 & 5.4 & $<1.0$ & 8.1 & 10.3 & 1.0 & na & 152 & 5.0 & na & 23.4 \\
\hline $\begin{array}{c}\text { Teton Pass, } \\
\text { Wyo. }\end{array}$ & $3 / 23 / 2000$ & E & 21.2 & 4.6 & 6.21 & 0.6 & 21.0 & 6.6 & 4.2 & 1.2 & 8.8 & 2.1 & 6.6 & 7.4 & 1.2 & na & 157 & na & na & 6.3 \\
\hline $\begin{array}{l}\text { Togwotee Pass, } \\
\text { Wyo. }\end{array}$ & $3 / 7 / 2000$ & E & 1.0 & 2.3 & 5.45 & 3.5 & 3.5 & $<1.7$ & 1.0 & $<0.4$ & 3.2 & $<1.0$ & 2.5 & 3.7 & 0.4 & na & 162 & na & na & 22.3 \\
\hline $\begin{array}{l}\text { Twenty-one } \\
\text { Mile, Mont. }\end{array}$ & 3/5/2000 & E & -3.1 & 4.0 & 5.17 & 6.8 & $<3.1$ & $<1.7$ & $<1.0$ & $<0.4$ & 7.5 & $<1.0$ & 4.6 & 8.6 & na & na & 122 & na & na & 3.6 \\
\hline $\begin{array}{l}\text { Univer- } \\
\text { sity Camp, } \\
\text { Colo. }\end{array}$ & 4/7/2000 & E & -2.8 & 3.6 & 5.01 & 9.8 & 9.5 & 2.5 & 1.5 & 1.2 & 7.5 & 1.1 & 9.4 & 10.6 & 0.7 & na & 215 & na & na & 20.3 \\
\hline $\begin{array}{l}\text { W5 (Glacier } \\
\text { NP), Mont. }\end{array}$ & $3 / 21 / 2000$ & E & -5.5 & 2.3 & 5.01 & 9.8 & $<3.1$ & $<1.7$ & 1.1 & 0.4 & 3.1 & $<1.0$ & 3.0 & 4.2 & 0.6 & 47 & 126 & na & na & 33.3 \\
\hline $\begin{array}{l}\text { W6 (Glacier } \\
\text { NP), Mont. }\end{array}$ & $3 / 22 / 2000$ & E & 2.0 & 2.5 & 5.15 & 7.1 & $<3.1$ & $<1.7$ & $<1.0$ & $<0.4$ & 4.6 & $<1.0$ & 4.0 & 5.0 & 0.8 & 87 & 215 & na & na & 3.1 \\
\hline $\begin{array}{l}\text { W7 (Glacier } \\
\text { NP), Mont. }\end{array}$ & $3 / 22 / 2000$ & E & -6.9 & 3.5 & 5.07 & 8.5 & $<3.1$ & $<1.7$ & 1.2 & $<0.4$ & 3.0 & $<1.0$ & 3.5 & 4.0 & 0.6 & 96 & 223 & na & na & 25.5 \\
\hline $\begin{array}{l}\text { W8 (Glacier } \\
\text { NP), Mont. }\end{array}$ & $3 / 22 / 2000$ & E & -9.6 & 3.2 & 4.98 & 10.5 & $<3.1$ & $<1.7$ & 1.0 & $<0.4$ & 3.1 & $<1.0$ & 3.7 & 5.0 & 0.5 & 110 & 297 & na & na & 25.1 \\
\hline $\begin{array}{l}\text { W9 (Glacier } \\
\text { NP), Mont. }\end{array}$ & $3 / 23 / 2000$ & E & -7.5 & 4.6 & 5.02 & 9.5 & $<3.1$ & $<1.7$ & 1.2 & $<0.4$ & 3.3 & $<1.0$ & 4.6 & 5.7 & 0.9 & 87 & 242 & na & na & 15.4 \\
\hline $\begin{array}{l}\text { W10 (Glacier } \\
\text { NP), Mont. }\end{array}$ & $3 / 23 / 2000$ & E & -9.1 & 3.3 & 4.97 & 10.7 & $<3.1$ & $<1.7$ & $<1.0$ & $<0.4$ & 4.0 & $<1.0$ & 3.9 & 5.2 & na & 74 & 219 & na & na & 23.6 \\
\hline $\begin{array}{l}\text { Wescott Falls, } \\
\text { Colo. }\end{array}$ & $3 / 28 / 2000$ & E & -7.3 & 5.3 & 4.88 & 13.2 & 5.5 & $<1.7$ & $<1.0$ & 0.5 & 5.6 & $<1.0$ & 7.9 & 10.8 & 0.4 & na & 73 & na & na & 14.0 \\
\hline $\begin{array}{l}\text { West Yellow- } \\
\text { stone, } \\
\text { Mont. }\end{array}$ & $3 / 5 / 2000$ & E & -0.7 & 5.1 & 5.27 & 5.4 & 7.5 & 2.5 & 1.4 & 0.9 & 9.0 & 1.1 & 5.7 & 10.5 & na & na & 64 & na & na & 21.1 \\
\hline $\begin{array}{l}\text { West Yellow- } \\
\text { stone (in } \\
\text { road), } \\
\text { Mont. }\end{array}$ & $3 / 5 / 2000$ & E & 4.5 & 3.5 & 5.59 & 2.6 & 5.5 & $<1.7$ & 2.5 & 0.7 & 11.7 & 4.7 & 2.1 & 6.7 & na & na & 20 & na & na & 12.0 \\
\hline $\begin{array}{l}\text { Wolf Creek } \\
\text { Pass, Colo. }\end{array}$ & $3 / 25 / 2000$ & E & -0.8 & 5.2 & 5.18 & 6.6 & 12.0 & 2.5 & 2.8 & 1.2 & 4.9 & 1.7 & 9.0 & 11.9 & 0.9 & na & 143 & 5.7 & na & 14.0 \\
\hline
\end{tabular}


[E, depth-integrated environmental; $\mathrm{ANC}$, acid neutralization capacity; $\mu \mathrm{eq} / \mathrm{L}$, microequivalents per liter; $\mu \mathrm{S} / \mathrm{cm}$, microsiemens per centimeter; $\mathrm{SC}$, specific conductance at $25^{\circ} \mathrm{C}$; $\mathrm{pH}$ values in standard units; $\mathrm{H}$, hydrogen; $\mathrm{Ca}$, calcium; $\mathrm{Mg}$, magnesium; $\mathrm{Na}$, sodium; $\mathrm{K}$, potassium; $\mathrm{NH}_{4}$, ammonium; $\mathrm{Cl}$, chloride; $\mathrm{SO}_{4}$, sulfate; $\mathrm{NO}_{3}$, nitrate; DOC, dissolved organic carbon; mg/L, milligrams per liter; $\mathrm{SWE}$, snow/water equivalent; cm, centimeters; $\delta^{34} \mathrm{~S}$, stable sulfur isotope ratio $\left({ }^{34} \mathrm{~S} /{ }^{22} \mathrm{~S}\right)$; Hg, mercury; ng/L, nanograms per liter; na, not analyzed; <, below reporting limits; NP, National Park; YNP, Yellowstone National Park; $\%$, percent]

\begin{tabular}{|c|c|c|c|c|c|c|c|c|c|c|c|c|c|c|c|c|c|c|c|c|}
\hline Site name & $\begin{array}{l}\text { Sample } \\
\text { date }\end{array}$ & $\begin{array}{c}\text { Sample } \\
\text { type }\end{array}$ & $\begin{array}{c}\text { ANC } \\
(\mu \mathrm{eq} / \mathrm{L})\end{array}$ & $\begin{array}{c}\mathrm{SC} \\
(\mu \mathrm{S} / \mathrm{cm})\end{array}$ & $\mathrm{pH}$ & $\begin{array}{c}H \\
(\mu e q / L)\end{array}$ & $\underset{(\mu \mathrm{eq} / \mathrm{L})}{\mathrm{Ca}}$ & $\begin{array}{c}\mathrm{Mg} \\
(\mu \mathrm{eq} / \mathrm{L})\end{array}$ & 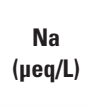 & $\begin{array}{c}K \\
(\mu \mathrm{eq} / \mathrm{L})\end{array}$ & $\begin{array}{c}\mathrm{NH}_{4} \\
(\mu \mathrm{eq} / \mathrm{L})\end{array}$ & $\underset{(\mu \mathrm{eq} / \mathrm{L})}{\mathrm{Cl}}$ & $\begin{array}{c}\mathrm{SO}_{4} \\
(\mu \mathrm{eq} / \mathrm{L})\end{array}$ & $\begin{array}{c}\mathrm{NO}_{3} \\
\text { ( } \mu \mathrm{eq} / \mathrm{L})\end{array}$ & $\begin{array}{l}\text { DOC } \\
\text { (mg/L) }\end{array}$ & $\begin{array}{l}\text { SWE } \\
\text { (cm) }\end{array}$ & $\begin{array}{l}\text { Snow } \\
\text { depth } \\
\text { (cm) }\end{array}$ & $\begin{array}{l}\delta^{34} S \\
\text { (per } \\
\text { mil) }\end{array}$ & $\begin{array}{c}\mathrm{Hg} \\
\text { (ng/L) }\end{array}$ & $\begin{array}{c}\text { lonic } \\
\text { balance } \\
(\%)\end{array}$ \\
\hline $\begin{array}{l}\text { Apgar Lookout } \\
\text { (A), Mont. }\end{array}$ & $3 / 14 / 2001$ & $\mathrm{E}$ & -9.2 & 5.8 & 5.03 & 9.3 & 4.6 & $<1.7$ & 2.2 & 0.8 & 12.3 & $<1.0$ & 10.4 & 14.2 & 1.0 & na & 119 & na & 1.3 & 8.5 \\
\hline $\begin{array}{l}\text { Apgar Lookout } \\
\text { (B), Mont. }\end{array}$ & $3 / 14 / 2001$ & E & -4.7 & 5.8 & 5.19 & 6.5 & 5.5 & $<1.7$ & 2.3 & 0.8 & 12.7 & $<1.0$ & 10.9 & 13.2 & 0.9 & 35 & 119 & 7.0 & 2.2 & 7.1 \\
\hline $\begin{array}{l}\text { Arapaho Creek, } \\
\text { Colo. }\end{array}$ & $3 / 13 / 2001$ & E & -18.9 & 9.5 & 4.85 & 14.1 & 5.8 & $<1.7$ & 1.8 & 0.4 & 5.1 & $<1.0$ & 7.3 & 21.7 & 0.4 & na & 89 & na & na & -3.2 \\
\hline Bear Lake, Colo. & $4 / 11 / 2001$ & E & -5.5 & 7.1 & 5.15 & 7.1 & 11.0 & 2.5 & 2.4 & 1.4 & 12.6 & $<1.0$ & 12.0 & 17.2 & 1.4 & na & 100 & na & na & 11.5 \\
\hline $\begin{array}{l}\text { Berthoud Pass, } \\
\text { Colo. }\end{array}$ & 4/3/2001 & E & -7.9 & 3.6 & 5.07 & 8.5 & 5.7 & $<1.7$ & 3.1 & 1.6 & 4.0 & 2.4 & 6.1 & 12.2 & 0.9 & na & 191 & 6.1 & na & 5.1 \\
\hline $\begin{array}{l}\text { Big Mountain, } \\
\text { Mont. }\end{array}$ & $3 / 9 / 2001$ & E & -9.3 & 4.8 & 5.05 & 8.9 & $<3.1$ & $<1.7$ & 1.5 & $<0.4$ & 4.3 & $<1.0$ & 6.0 & 8.5 & 0.5 & na & 132 & 5.6 & 1.2 & 0.9 \\
\hline Big Sky, Mont. & $3 / 17 / 2001$ & E & -3.0 & 3.5 & 5.35 & 4.5 & 4.0 & $<1.7$ & $<1.0$ & 0.5 & 4.2 & $<1.0$ & 4.3 & 6.6 & 0.5 & na & 124 & 5.6 & na & 9.3 \\
\hline $\begin{array}{l}\text { Bowman } 1 \\
\quad \text { (Glacier NP), } \\
\text { Mont. }\end{array}$ & $3 / 8 / 2001$ & E & -10.6 & 5.1 & 5.04 & 9.1 & $<3.1$ & $<1.7$ & 1.1 & $<0.4$ & 2.1 & $<1.0$ & 4.1 & 8.5 & 0.4 & 23 & 76 & na & na & -0.6 \\
\hline $\begin{array}{l}\text { Bowman } 2 \\
\quad \text { (Glacier NP), } \\
\text { Mont. }\end{array}$ & $3 / 8 / 2001$ & E & -10.2 & 4.6 & 5.10 & 7.9 & $<3.1$ & $<1.7$ & 1.9 & $<0.4$ & 2.4 & $<1.0$ & 6.1 & 7.3 & 0.6 & 41 & 122 & na & na & -4.4 \\
\hline $\begin{array}{l}\text { Brooklyn Lake, } \\
\text { Wyo. }\end{array}$ & $3 / 27 / 2001$ & E & -1.9 & 4.0 & 5.48 & 3.3 & 7.3 & $<1.7$ & 1.6 & 1.1 & 4.6 & $<1.0$ & 6.1 & 10.8 & 0.9 & na & 165 & 7.1 & na & 2.9 \\
\hline Brumley, Colo. & $3 / 26 / 2001$ & E & -4.5 & 6.2 & 5.18 & 6.6 & 12.0 & 2.5 & 5.2 & 1.4 & 14.5 & 1.2 & 13.7 & 15.2 & 1.0 & na & 110 & na & na & 16.7 \\
\hline $\begin{array}{l}\text { Buffalo Pass, } \\
\text { Colo. }\end{array}$ & $4 / 4 / 2001$ & E & -11.6 & 6.1 & 4.98 & 10.5 & 7.0 & $<1.7$ & 2.7 & 0.5 & 6.5 & $<1.0$ & 11.1 & 13.1 & na & na & 309 & 6.1 & 2.0 & 5.7 \\
\hline $\begin{array}{l}\text { Cameron Pass, } \\
\text { Colo. }\end{array}$ & $4 / 5 / 2001$ & E & -6.1 & 6.1 & 5.21 & 6.2 & 6.0 & $<1.7$ & 1.9 & 0.6 & 6.5 & $<1.0$ & 8.1 & 11.8 & 0.5 & na & 147 & na & 1.5 & 2.9 \\
\hline Canyon, Wyo. & $3 / 1 / 2001$ & E & -5.1 & 4.1 & 5.23 & 5.9 & $<3.1$ & $<1.7$ & 1.0 & $<0.4$ & 6.1 & $<1.0$ & 4.4 & 9.3 & 0.4 & na & 88 & 3.1 & 0.7 & -2.6 \\
\hline $\begin{array}{l}\text { Chief Joseph Pass, } \\
\text { Mont. }\end{array}$ & $3 / 16 / 2001$ & E & -5.6 & 3.4 & 5.26 & 5.5 & 4.5 & $<1.7$ & 1.1 & 1.3 & 2.8 & $<1.0$ & 4.1 & 5.5 & 1.2 & na & 112 & 5.3 & 3.4 & 22.2 \\
\hline Daisy Pass, Mont. & $3 / 6 / 2001$ & E & -4.0 & 3.7 & 5.25 & 5.6 & 3.5 & $<1.7$ & $<1.0$ & 0.9 & 4.9 & $<1.0$ & 4.9 & 7.5 & 0.8 & na & 135 & 4.9 & 1.8 & 9.2 \\
\hline $\begin{array}{l}\text { Deadman Pass, } \\
\text { Colo. }\end{array}$ & $3 / 28 / 2001$ & E & -4.9 & 6.8 & 5.21 & 6.2 & 8.8 & 1.7 & 1.9 & 1.4 & 6.8 & $<1.0$ & 9.0 & 14.6 & 0.9 & na & 145 & na & na & 6.4 \\
\hline $\begin{array}{l}\text { Dinah Shore } 1 \\
\text { (Glacier NP), } \\
\text { Mont. }\end{array}$ & $3 / 9 / 2001$ & E & -10.7 & 4.4 & 5.17 & 6.8 & 3.1 & $<1.7$ & 2.0 & 0.4 & 3.5 & $<1.0$ & 4.9 & 8.2 & 0.5 & 24 & 86 & na & na & 9.4 \\
\hline
\end{tabular}


Table 10. Selected chemical and physical data for Rocky Mountain snowpack samples for 2001.—Continued

[E, depth-integrated environmental; ANC, acid neutralization capacity; $\mu \mathrm{eq} / \mathrm{L}$, microequivalents per liter; $\mu \mathrm{S} / \mathrm{cm}$, microsiemens per centimeter; $\mathrm{SC}$, specific conductance at $25^{\circ} \mathrm{C} ; \mathrm{pH}$ values in standard units; $\mathrm{H}$, hydrogen; Ca, calcium; $\mathrm{Mg}$, magnesium; $\mathrm{Na}$, sodium; $\mathrm{K}$, potassium; $\mathrm{NH}_{4}$, ammonium; $\mathrm{Cl}$, chloride; $\mathrm{SO}_{4}$, sulfate; $\mathrm{NO}_{3}$, nitrate; DOC, dissolved organic carbon; mg/L, milligrams per liter; $\mathrm{SWE}$, snow/water equivalent; cm, centimeters; $\delta^{34} \mathrm{~S}$, stable sulfur isotope ratio $\left({ }^{34} \mathrm{~S} /{ }^{2} \mathrm{~S}\right) ; \mathrm{Hg}$, mercury; ng/L, nanograms per liter; na, not analyzed; <, below reporting limits; NP, National Park; YNP, Yellowstone National Park; $\%$, percent]

\begin{tabular}{|c|c|c|c|c|c|c|c|c|c|c|c|c|c|c|c|c|c|c|c|c|}
\hline Site name & $\begin{array}{l}\text { Sample } \\
\text { date }\end{array}$ & $\begin{array}{l}\text { Sample } \\
\text { type }\end{array}$ & $\begin{array}{c}\text { ANC } \\
(\mu \mathrm{eq} / \mathrm{L})\end{array}$ & $\begin{array}{c}S C \\
(\mu \mathrm{S} / \mathrm{cm})\end{array}$ & $\mathrm{pH}$ & $\begin{array}{c}\text { H } \\
(\mu \mathrm{eq} / \mathrm{L})\end{array}$ & $\begin{array}{c}\text { Ca } \\
(\mu \mathrm{eq} / \mathrm{L})\end{array}$ & $\underset{(\mu \mathrm{eq} / \mathrm{L})}{\mathrm{Mg}}$ & $\underset{(\mu \mathrm{eq} / \mathrm{L})}{\mathrm{Na}}$ & $\begin{array}{c}K \\
(\mu \mathrm{eq} / L)\end{array}$ & 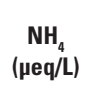 & $\begin{array}{c}\text { CI } \\
(\mu \mathrm{eq} / \mathrm{L})\end{array}$ & $\begin{array}{c}\mathrm{SO}_{4} \\
(\mu \mathrm{eq} / \mathrm{L})\end{array}$ & $\begin{array}{c}\mathrm{NO}_{3} \\
(\mu \mathrm{eq} / \mathrm{L})\end{array}$ & $\begin{array}{l}\text { DOC } \\
(\mathrm{mg} / \mathrm{L})\end{array}$ & $\begin{array}{l}\text { SWE } \\
\text { (cm) }\end{array}$ & $\begin{array}{l}\text { Snow } \\
\text { depth } \\
(\mathrm{cm})\end{array}$ & $\begin{array}{l}\delta^{34} S \\
\text { (per } \\
\text { mil) }\end{array}$ & $\begin{array}{c}\mathrm{Hg} \\
\text { (ng/L) }\end{array}$ & $\begin{array}{c}\text { lonic } \\
\text { balance } \\
(\%)\end{array}$ \\
\hline $\begin{array}{l}\text { Dinah Shore } 2 \\
\quad \text { (Glacier NP), } \\
\text { Mont. }\end{array}$ & 3/9/2001 & E & -10.5 & 3.5 & 5.12 & 7.6 & $<3.1$ & $<1.7$ & 1.0 & 0.6 & 2.1 & $<1.0$ & 4.1 & 5.4 & 0.8 & 68 & 210 & na & na & 8.3 \\
\hline $\begin{array}{l}\text { Divide Peak, } \\
\text { Wyo. }\end{array}$ & $3 / 14 / 2001$ & E & -7.3 & 6.2 & 5.22 & 6.0 & 15.0 & 3.3 & 7.0 & 0.6 & 6.4 & 1.6 & 12.5 & 20.1 & 0.7 & na & 105 & na & 1.8 & 5.5 \\
\hline Dry Lake, Colo. & $3 / 15 / 2001$ & E & -20.8 & 9.4 & 4.78 & 16.6 & 7.5 & $<1.7$ & 1.8 & 0.8 & 6.6 & $<1.0$ & 10.3 & 20.7 & $<0.4$ & na & 137 & 5.6 & 0.9 & 3.5 \\
\hline $\begin{array}{l}\text { Dunckley Pass, } \\
\text { Colo. }\end{array}$ & 4/3/2001 & E & 2.4 & 4.4 & 5.66 & 2.2 & 12.5 & 2.5 & 2.0 & 2.4 & 5.7 & $<1.0$ & 8.1 & 12.6 & 0.7 & na & 170 & na & 2.0 & 8.2 \\
\hline $\begin{array}{l}\text { East Inlet Grand } \\
\text { Lake, Colo. }\end{array}$ & $3 / 13 / 2001$ & E & -10.4 & 7.6 & 5.01 & 9.8 & 11.5 & 3.3 & 3.5 & 4.4 & 6.4 & 1.2 & 10.5 & 20.1 & 1.6 & na & 49 & na & na & 10.0 \\
\hline Elk River, Colo. & $3 / 15 / 2001$ & E & -11.0 & 7.7 & 5.00 & 10.0 & 11.5 & $<1.7$ & 4.0 & 1.0 & 5.1 & 1.1 & 10.4 & 17.9 & 0.6 & na & 136 & na & na & 3.6 \\
\hline $\begin{array}{l}\text { Elkhart Park, } \\
\text { Wyo. }\end{array}$ & $3 / 11 / 2001$ & E & -8.7 & 5.2 & 5.07 & 8.5 & 4.0 & $<1.7$ & 1.3 & $<0.4$ & 3.6 & $<1.0$ & 5.9 & 10.3 & 0.7 & na & 111 & 6.4 & na & 3.4 \\
\hline $\begin{array}{l}\text { Four Mile } \\
\text { Meadow, Wyo. }\end{array}$ & $3 / 12 / 2001$ & E & -7.2 & 3.8 & 5.25 & 5.6 & 6.5 & 2.5 & 1.9 & 0.6 & 5.9 & $<1.0$ & 4.9 & 10.1 & 0.6 & na & 63 & na & na & 21.0 \\
\hline $\begin{array}{l}\text { Fremont Pass, } \\
\text { Colo. }\end{array}$ & 4/9/2001 & E & -5.1 & 3.9 & 5.31 & 4.9 & 5.5 & $<1.7$ & 1.4 & 0.6 & 2.9 & $<1.0$ & 4.6 & 8.6 & 0.5 & na & 120 & 5.4 & 5.9 & 7.1 \\
\hline $\begin{array}{l}\text { Garnet Canyon, } \\
\text { Wyo. }\end{array}$ & $3 / 19 / 2001$ & $\mathrm{E}$ & -5.6 & 4.3 & 5.33 & 4.7 & 6.0 & $<1.7$ & 2.1 & 1.2 & 8.4 & $<1.0$ & 7.2 & 10.1 & 0.7 & na & 136 & 5.4 & na & 12.8 \\
\hline $\begin{array}{l}\text { Grand Mesa, } \\
\text { Colo. }\end{array}$ & $3 / 10 / 2001$ & E & -4.7 & 4.9 & 5.39 & 4.1 & 13.0 & 2.5 & 2.2 & 1.2 & 7.4 & $<1.0$ & 9.8 & 14.8 & 0.7 & na & 126 & na & 5.1 & 10.2 \\
\hline $\begin{array}{l}\text { Granite Park, } \\
\text { Mont. }\end{array}$ & $3 / 10 / 2001$ & E & -11.6 & 5.0 & 5.06 & 8.7 & $<3.1$ & $<1.7$ & 1.5 & $<0.4$ & 4.0 & $<1.0$ & 10.5 & 7.7 & 0.4 & 44 & 127 & na & na & -12.2 \\
\hline $\begin{array}{l}\text { Granite Pass, } \\
\text { Mont. }\end{array}$ & $3 / 16 / 2001$ & E & -2.8 & 3.6 & 5.39 & 4.1 & 5.5 & $<1.7$ & 1.7 & 1.1 & 3.7 & $<1.0$ & 5.0 & 5.4 & 0.9 & na & 121 & 5.2 & 4.4 & 21.8 \\
\hline $\begin{array}{l}\text { Green Ridge, } \\
\text { Colo. }\end{array}$ & $3 / 12 / 2001$ & E & -19.0 & 7.3 & 4.89 & 12.9 & 7.1 & $<1.7$ & 1.8 & 0.9 & 4.0 & 1.0 & 7.2 & 19.5 & 1.1 & na & 53 & na & na & -1.9 \\
\hline $\begin{array}{l}\text { Gypsum Creek, } \\
\text { Wyo. }\end{array}$ & $3 / 11 / 2001$ & E & -5.9 & 5.0 & 5.17 & 6.8 & 7.5 & 2.5 & 1.9 & 1.6 & 3.7 & $<1.0$ & 6.2 & 11.7 & 1.2 & na & 65 & na & na & 14.4 \\
\hline $\begin{array}{l}\text { Halfmoon Creek, } \\
\text { Colo. }\end{array}$ & $4 / 5 / 2001$ & E & -5.2 & 4.9 & 5.24 & 5.8 & 10.0 & 2.1 & 1.5 & 1.8 & 5.7 & $<1.0$ & 7.1 & 10.0 & 1.8 & na & 69 & na & na & 22.0 \\
\hline $\begin{array}{l}\text { Hopewell, } \\
\text { N. Mex. }\end{array}$ & $3 / 16 / 2001$ & E & -12.0 & 6.9 & 5.02 & 9.5 & 8.0 & $<1.7$ & 2.4 & 0.8 & 6.3 & 1.1 & 8.1 & 14.3 & 0.5 & na & 150 & 4.3 & 2.9 & 6.9 \\
\hline Kings Hill, Mont. & 3/7/2001 & E & -6.9 & 5.5 & 5.15 & 7.1 & 7.0 & $<1.7$ & 1.4 & 2.3 & 7.4 & $<1.0$ & 9.7 & 10.0 & 1.4 & na & 106 & 8.7 & na & 12.0 \\
\hline Lake Irene, Colo. & $4 / 2 / 2001$ & E & -9.7 & 5.5 & 5.08 & 8.3 & 6.5 & $<1.7$ & 2.3 & 0.5 & 5.5 & $<1.0$ & 8.4 & 13.0 & 0.5 & na & 183 & 6.0 & 2.8 & 3.8 \\
\hline
\end{tabular}


[E, depth-integrated environmental; ANC, acid neutralization capacity; $\mu \mathrm{eq} / \mathrm{L}$, microequivalents per liter; $\mu \mathrm{S} / \mathrm{cm}$, microsiemens per centimeter; $\mathrm{SC}$, specific conductance at $25^{\circ} \mathrm{C} ; \mathrm{pH}$ values in standard units; $\mathrm{H}$, hydrogen; $\mathrm{Ca}$, calcium; $\mathrm{Mg}$, magnesium; $\mathrm{Na}$, sodium; $\mathrm{K}$, potassium; $\mathrm{NH}_{4}$, ammonium; $\mathrm{Cl}$, chloride; $\mathrm{SO}_{4}$, sulfate; $\mathrm{NO}_{3}$, nitrate; DOC, dissolved organic carbon; mg/L, milligrams per liter; $\mathrm{SWE}$, snow/water equivalent; cm, centimeters; $\delta^{34} \mathrm{~S}$, stable sulfur isotope ratio $\left({ }^{34} \mathrm{~S} /{ }^{22} \mathrm{~S}\right)$; Hg, mercury; ng/L, nanograms per liter; na, not analyzed; <, below reporting limits; NP, National Park; YNP, Yellowstone National Park; $\%$, percent]

\begin{tabular}{|c|c|c|c|c|c|c|c|c|c|c|c|c|c|c|c|c|c|c|c|c|}
\hline Site name & $\begin{array}{c}\text { Sample } \\
\text { date }\end{array}$ & $\begin{array}{c}\text { Sample } \\
\text { type }\end{array}$ & $\begin{array}{c}\text { ANC } \\
(\mu \mathrm{eq} / \mathrm{L})\end{array}$ & $\begin{array}{c}\text { SC } \\
(\mu \mathrm{S} / \mathrm{cm})\end{array}$ & pH & 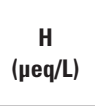 & $\begin{array}{c}\text { Ca } \\
(\mu \mathrm{eq} / \mathrm{L})\end{array}$ & $\begin{array}{c}\mathrm{Mg} \\
(\mu \mathrm{eq} / \mathrm{L})\end{array}$ & $\begin{array}{c}\mathrm{Na} \\
(\mu \mathrm{eq} / \mathrm{L})\end{array}$ & $\begin{array}{c}K \\
(\mu e q / L)\end{array}$ & $\begin{array}{c}\mathrm{NH}_{4} \\
(\mu \mathrm{eq} / \mathrm{L})\end{array}$ & $\begin{array}{c}\mathrm{Cl} \\
(\mu \mathrm{eq} / \mathrm{L})\end{array}$ & $\underset{(\mu \mathrm{eq} / \mathrm{L})}{\mathbf{S O}_{4}}$ & $\begin{array}{c}\mathrm{NO}_{3} \\
(\mu \mathrm{eq} / \mathrm{L})\end{array}$ & $\begin{array}{l}\text { DOC } \\
\text { (mg/L) }\end{array}$ & $\begin{array}{l}\text { SWE } \\
\text { (cm) }\end{array}$ & $\begin{array}{l}\text { Snow } \\
\text { depth } \\
\text { (cm) }\end{array}$ & $\begin{array}{l}\delta^{34} S \\
\text { (per } \\
\text { mil) }\end{array}$ & $\begin{array}{c}\mathrm{Hg} \\
\text { (ng/L) }\end{array}$ & $\begin{array}{c}\text { lonic } \\
\text { balance } \\
(\%)\end{array}$ \\
\hline $\begin{array}{l}\text { Lewis Lake } \\
\text { Divide, Wyo. }\end{array}$ & $3 / 1 / 2001$ & E & -4.0 & 4.2 & 5.35 & 4.5 & $<3.1$ & $<1.7$ & 1.1 & $<0.4$ & 10.6 & $<1.0$ & 5.4 & 9.8 & 0.4 & na & 174 & na & 1.0 & 3.0 \\
\hline Lionshead, Mont. & $3 / 4 / 2001$ & E & -6.9 & 5.6 & 5.17 & 6.8 & 4.5 & $<1.7$ & 1.2 & 0.9 & 14.3 & 1.0 & 7.7 & 15.7 & 0.8 & na & 112 & 5.0 & na & 6.1 \\
\hline $\begin{array}{l}\text { Loch Vale Forest, } \\
\text { Colo. }\end{array}$ & $4 / 10 / 2001$ & E & -7.1 & 5.4 & 5.17 & 6.8 & 11.0 & 2.5 & 2.4 & 2.8 & 6.0 & 1.0 & 10.0 & 14.6 & 1.9 & na & 194 & 5.8 & 5.2 & 10.1 \\
\hline $\begin{array}{l}\text { Loch Vale } \\
\quad \text { Meadow, Colo. }\end{array}$ & $4 / 10 / 2001$ & E & -6.9 & 5.2 & 5.18 & 6.6 & 8.5 & $<1.7$ & 1.8 & 0.5 & 7.3 & 3.7 & 9.3 & 13.4 & 0.5 & na & 141 & na & na & -3.3 \\
\hline $\begin{array}{l}\text { Loveland Pass, } \\
\text { Colo. }\end{array}$ & $4 / 27 / 2001$ & E & 3.3 & 3.6 & 5.83 & 1.5 & 13.5 & 3.3 & 2.4 & 0.5 & 5.9 & 2.1 & 6.1 & 9.2 & $<0.4$ & na & 190 & 5.6 & na & 13.2 \\
\hline Lynx Pass, Colo. & $3 / 14 / 2001$ & E & -6.2 & 5.6 & 5.22 & 6.0 & 8.5 & $<1.7$ & 1.8 & 0.9 & 8.4 & $<1.0$ & 6.2 & 15.9 & 0.7 & na & 111 & 6.2 & na & 7.5 \\
\hline $\begin{array}{l}\text { Marion Lake } 1 \\
\text { (Glacier NP), } \\
\text { Mont. }\end{array}$ & $3 / 13 / 2001$ & E & -4.9 & 5.0 & 5.29 & 5.1 & $<3.1$ & $<1.7$ & 1.6 & 0.7 & 4.8 & $<1.0$ & 6.9 & 8.1 & 0.7 & 42 & 159 & na & na & -10.2 \\
\hline $\begin{array}{l}\text { Marion Lake } 2 \\
\quad \text { (Glacier NP), } \\
\text { Mont. }\end{array}$ & $3 / 13 / 2001$ & E & -8.2 & 4.8 & 5.11 & 7.8 & 3.5 & $<1.7$ & 3.1 & 1.8 & 3.3 & 1.2 & 5.8 & 6.7 & 1.3 & 56 & 169 & na & na & 17.1 \\
\hline Molas Lake, Colo. & $3 / 28 / 2001$ & E & -4.0 & 4.3 & 5.30 & 5.0 & 8.0 & $<1.7$ & 1.3 & 0.5 & 2.7 & $<1.0$ & 5.5 & 10.6 & 0.5 & na & 165 & 4.9 & na & 4.2 \\
\hline $\begin{array}{l}\text { Monarch Pass, } \\
\text { Colo. }\end{array}$ & $3 / 26 / 2001$ & E & -2.2 & 3.9 & 5.40 & 4.0 & 7.0 & $<1.7$ & 1.0 & 0.6 & 5.7 & $<1.0$ & 5.4 & 9.7 & 0.9 & na & 113 & 4.7 & 4.0 & 9.5 \\
\hline $\begin{array}{l}\text { Monida Pass, } \\
\text { Mont. }\end{array}$ & $3 / 5 / 2001$ & E & -9.1 & 7.0 & 5.11 & 7.8 & 6.5 & $<1.7$ & 2.8 & 0.5 & 13.7 & 2.5 & 5.5 & 23.1 & 0.6 & na & 104 & na & na & 0.1 \\
\hline $\begin{array}{l}\text { Mount Belmont, } \\
\text { Mont. }\end{array}$ & $3 / 8 / 2001$ & E & -6.9 & 6.6 & 5.13 & 7.4 & 9.5 & 2.5 & 2.6 & 2.9 & 4.6 & 1.6 & 11.3 & 12.6 & 1.9 & na & 59 & na & na & 7.2 \\
\hline $\begin{array}{l}\text { Napa } 1 \text { (Glacier } \\
\text { NP), Mont. }\end{array}$ & $3 / 8 / 2001$ & E & -8.6 & 5.5 & 5.21 & 6.2 & 3.1 & $<1.7$ & 1.3 & 1.2 & 3.2 & $<1.0$ & 5.0 & 9.0 & 1.1 & 17 & 65 & na & 1.9 & 3.2 \\
\hline $\begin{array}{l}\text { Napa } 2 \text { (Glacier } \\
\text { NP), Mont. }\end{array}$ & $3 / 8 / 2001$ & E & -8.5 & 4.3 & 5.24 & 5.8 & 3.5 & $<1.7$ & 1.5 & $<0.4$ & 2.6 & $<1.0$ & 3.9 & 6.6 & 0.4 & 40 & 132 & na & 0.9 & 11.9 \\
\hline $\begin{array}{l}\text { Niwot Snotel, } \\
\text { Colo. }\end{array}$ & $3 / 26 / 2001$ & E & -6.7 & 7.8 & 5.11 & 7.8 & 10.0 & 2.5 & 3.0 & 1.1 & 16.9 & 1.4 & 14.4 & 20.0 & 1.0 & na & 97 & 5.9 & na & 7.0 \\
\hline $\begin{array}{l}\text { Noisy Basin, } \\
\text { Mont. }\end{array}$ & $3 / 8 / 2001$ & E & -10.5 & 5.6 & 4.99 & 10.2 & $<3.1$ & $<1.7$ & 1.4 & 0.6 & 4.2 & $<1.0$ & 6.6 & 8.9 & 0.7 & na & 203 & 5.6 & na & 3.1 \\
\hline $\begin{array}{l}\text { North Inlet Grand } \\
\text { Lake, Colo. }\end{array}$ & $4 / 4 / 2001$ & E & -10.4 & 7.2 & 5.08 & 8.3 & 7.1 & $<1.7$ & 2.0 & 1.2 & 5.6 & $<1.0$ & 7.6 & 15.7 & 1.0 & na & 82 & na & na & 1.7 \\
\hline Old Battle, Wyo. & $3 / 14 / 2001$ & E & -10.2 & 6.5 & 5.06 & 8.7 & 9.0 & 2.5 & 4.9 & 0.8 & 7.0 & 1.6 & 12.1 & 16.1 & 0.7 & na & 202 & 7.2 & 2.9 & 4.8 \\
\hline $\begin{array}{l}\text { Old Faithful (in } \\
\text { road), Wyo. }\end{array}$ & $3 / 3 / 2001$ & E & 32.3 & 14.7 & 6.14 & 0.7 & 58.4 & 9.1 & 10.3 & 6.0 & 26.9 & 12.5 & 29.9 & 14.8 & 6.2 & na & 15 & na & na & 10.9 \\
\hline
\end{tabular}


Table 10. Selected chemical and physical data for Rocky Mountain snowpack samples for 2001.—Continued

[E, depth-integrated environmental; ANC, acid neutralization capacity; $\mu \mathrm{eq} / \mathrm{L}$, microequivalents per liter; $\mu \mathrm{S} / \mathrm{cm}$, microsiemens per centimeter; $\mathrm{SC}$, specific conductance at $25^{\circ} \mathrm{C} ; \mathrm{pH}$ values in standard units; $\mathrm{H}$, hydrogen; Ca, calcium; $\mathrm{Mg}$, magnesium; $\mathrm{Na}$, sodium; $\mathrm{K}$, potassium; $\mathrm{NH}_{4}$, ammonium; $\mathrm{Cl}$, chloride; $\mathrm{SO}_{4}$, sulfate; $\mathrm{NO}_{3}$, nitrate; DOC, dissolved organic carbon; mg/L, milligrams per liter; $\mathrm{SWE}$, snow/water equivalent; cm, centimeters; $\delta^{34} \mathrm{~S}$, stable sulfur isotope ratio $\left({ }^{34} \mathrm{~S} /{ }^{2} \mathrm{~S}\right) ; \mathrm{Hg}$, mercury; ng/L, nanograms per liter; na, not analyzed; <, below reporting limits; NP, National Park; YNP, Yellowstone National Park; $\%$, percent]

\begin{tabular}{|c|c|c|c|c|c|c|c|c|c|c|c|c|c|c|c|c|c|c|c|c|}
\hline Site name & $\begin{array}{l}\text { Sample } \\
\text { date }\end{array}$ & $\begin{array}{l}\text { Sample } \\
\text { type }\end{array}$ & $\begin{array}{c}\text { ANC } \\
(\mu \mathrm{eq} / \mathrm{L})\end{array}$ & $\begin{array}{c}\mathrm{SC} \\
(\mu \mathrm{S} / \mathrm{cm})\end{array}$ & $\mathrm{pH}$ & $\begin{array}{c}H \\
(\mu \mathrm{eq} / \mathrm{L})\end{array}$ & $\begin{array}{c}\mathrm{Ca} \\
(\mu \mathrm{eq} / \mathrm{L})\end{array}$ & $\underset{(\mu \mathrm{eq} / \mathrm{L})}{\mathrm{Mg}}$ & $\begin{array}{c}\mathrm{Na} \\
(\mu \mathrm{eq} / \mathrm{L})\end{array}$ & $\begin{array}{c}K \\
(\mu e q / L)\end{array}$ & $\begin{array}{c}\mathrm{NH}_{4} \\
(\mu \mathrm{eq} / \mathrm{L})\end{array}$ & $\begin{array}{c}\text { CI } \\
(\mu \mathrm{eq} / \mathrm{L})\end{array}$ & $\begin{array}{c}\mathrm{SO}_{4} \\
(\mu \mathrm{eq} / \mathrm{L})\end{array}$ & $\begin{array}{c}\mathrm{NO}_{3} \\
(\mu \mathrm{eq} / \mathrm{L})\end{array}$ & $\begin{array}{c}\text { DOC } \\
\text { (mg/L) }\end{array}$ & $\begin{array}{l}\text { SWE } \\
\text { (cm) }\end{array}$ & $\begin{array}{l}\text { Snow } \\
\text { depth } \\
(\mathrm{cm})\end{array}$ & $\begin{array}{l}\delta^{34} S \\
\text { (per } \\
\text { mil) }\end{array}$ & $\underset{(n g / L)}{\mathrm{Hg}}$ & $\begin{array}{c}\text { lonic } \\
\text { balance } \\
(\%)\end{array}$ \\
\hline $\begin{array}{l}\text { Old Faithful Fire } \\
\text { Road, Wyo. }\end{array}$ & $3 / 3 / 2001$ & $\mathrm{E}$ & -8.0 & 4.5 & 5.19 & 6.5 & 4.0 & $<1.7$ & 1.5 & 0.4 & 10.6 & $<1.0$ & 6.1 & 12.7 & 0.7 & na & 70 & na & na & 10.0 \\
\hline $\begin{array}{l}\text { Old Faithful West } \\
\text { Lot, Wyo. }\end{array}$ & $3 / 3 / 2001$ & E & 26.1 & 21.8 & 6.25 & 0.6 & 44.1 & 6.0 & 67.4 & 10.6 & 26.2 & 75.9 & 24.4 & 15.2 & 4.8 & na & 33 & na & 5.8 & 4.4 \\
\hline $\begin{array}{l}\text { Phantom Valley, } \\
\text { Colo. }\end{array}$ & $3 / 13 / 2001$ & E & -13.1 & 9.1 & 4.90 & 12.6 & 9.0 & $<1.7$ & 2.6 & 0.8 & 6.4 & $<1.0$ & 10.8 & 18.5 & 0.8 & na & 83 & na & na & 3.3 \\
\hline $\begin{array}{l}\text { Rabbit Ears 1, } \\
\text { Colo. }\end{array}$ & 4/3/2001 & E & -7.8 & 5.8 & 5.16 & 6.9 & 7.0 & $<1.7$ & 2.8 & $<0.4$ & 6.2 & $<1.0$ & 10.2 & 13.7 & 0.4 & na & 225 & 6.8 & 1.5 & -2.2 \\
\hline $\begin{array}{l}\text { Rabbit Ears 2, } \\
\text { Colo. }\end{array}$ & 4/3/2001 & E & -11.0 & 5.9 & 5.02 & 9.5 & 6.5 & $<1.7$ & 2.7 & $<0.4$ & 5.3 & $<1.0$ & 9.5 & 13.9 & 0.4 & na & 215 & 6.6 & na & 1.3 \\
\hline $\begin{array}{l}\text { Red Mountain } \\
\text { Pass, Colo. }\end{array}$ & $3 / 28 / 2001$ & E & -1.4 & 4.0 & 5.43 & 3.7 & 9.0 & $<1.7$ & 1.4 & 1.3 & 1.9 & $<1.0$ & 5.1 & 9.2 & 0.9 & na & 174 & na & 1.1 & 9.7 \\
\hline $\begin{array}{l}\text { Red Mountain, } \\
\text { Mont. }\end{array}$ & $3 / 16 / 2001$ & E & -2.0 & 3.8 & 5.50 & 3.2 & 8.5 & 2.5 & 1.3 & 0.9 & 5.5 & $<1.0$ & 6.0 & 9.5 & 1.1 & na & 125 & na & na & 17.0 \\
\hline $\begin{array}{l}\text { Rendezvous } \\
\text { Mountain, } \\
\text { Wyo. }\end{array}$ & $3 / 20 / 2001$ & E & -3.5 & 3.3 & 5.39 & 4.1 & $<3.1$ & $<1.7$ & $<1.0$ & 0.5 & 6.8 & $<1.0$ & 5.9 & 7.0 & 0.5 & na & 201 & na & 1.2 & -6.5 \\
\hline $\begin{array}{l}\text { Slumgullion Pass, } \\
\text { Colo. }\end{array}$ & $3 / 27 / 2001$ & E & 3.5 & 4.8 & 5.68 & 2.1 & 11.5 & 3.3 & 2.3 & 1.9 & 2.2 & 1.0 & 5.2 & 7.3 & 1.0 & na & 120 & na & na & 15.5 \\
\hline $\begin{array}{l}\text { Smith Creek } 1 \\
\text { (Glacier NP), } \\
\text { Mont. }\end{array}$ & $3 / 10 / 2001$ & E & -7.4 & 4.0 & 5.27 & 5.4 & 4.4 & $<1.7$ & 1.4 & 1.8 & 2.6 & $<1.0$ & 4.9 & 7.5 & 1.3 & 22 & 74 & na & 2.2 & 11.2 \\
\hline $\begin{array}{l}\text { Smith Creek } 2 \\
\quad \text { (Glacier NP), } \\
\text { Mont. }\end{array}$ & $3 / 10 / 2001$ & $\mathrm{E}$ & -2.6 & 3.4 & 5.44 & 3.6 & 5.9 & $<1.7$ & $<1.0$ & 0.8 & 2.8 & $<1.0$ & 3.5 & 6.1 & 0.6 & 42 & 142 & na & 0.8 & 15.3 \\
\hline $\begin{array}{l}\text { Snow Bowl, } \\
\text { Mont. }\end{array}$ & $3 / 10 / 2001$ & E & -6.5 & 4.4 & 5.12 & 7.6 & $<3.1$ & $<1.7$ & 1.1 & 1.1 & 2.7 & $<1.0$ & 5.2 & 5.6 & 1.0 & na & 159 & na & na & 6.7 \\
\hline $\begin{array}{l}\text { South Entrance } \\
\text { YNP (in road), } \\
\text { Wyo. }\end{array}$ & $3 / 2 / 2001$ & E & -3.2 & 5.5 & 5.36 & 4.4 & 5.6 & $<1.7$ & 1.7 & 2.5 & 14.0 & 1.0 & 8.4 & 12.0 & 1.3 & na & 68 & na & na & 13.6 \\
\hline $\begin{array}{l}\text { South Entrance } \\
\text { YNP, Wyo. }\end{array}$ & $3 / 1 / 2001$ & E & -1.7 & 5.2 & 5.45 & 3.5 & 3.7 & $<1.7$ & 1.8 & 1.2 & 12.2 & $<1.0$ & 6.3 & 12.5 & 0.7 & na & 104 & na & na & 8.9 \\
\hline South Pass, Wyo. & $3 / 13 / 2001$ & $\mathrm{E}$ & -12.4 & 7.2 & 4.99 & 10.2 & 6.0 & $<1.7$ & 1.8 & 0.4 & 5.1 & $<1.0$ & 9.9 & 14.3 & 0.7 & na & 77 & na & na & -1.5 \\
\hline $\begin{array}{l}\text { Spring Gulch, } \\
\text { Mont. }\end{array}$ & $3 / 8 / 2001$ & E & -5.8 & 5.6 & 5.17 & 6.8 & 7.5 & $<1.7$ & 1.3 & $<0.4$ & 5.9 & 3.9 & 9.1 & 13.8 & 0.9 & na & 48 & 4.8 & na & -11.2 \\
\hline $\begin{array}{l}\text { Sunlight Peak, } \\
\text { Colo. }\end{array}$ & $3 / 29 / 2001$ & E & -1.7 & 4.6 & 5.37 & 4.3 & 9.0 & $<1.7$ & 1.8 & 0.6 & 6.5 & $<1.0$ & 7.3 & 12.3 & 0.4 & na & 179 & 5.4 & na & 6.0 \\
\hline
\end{tabular}


[E, depth-integrated environmental; ANC, acid neutralization capacity; $\mu \mathrm{eq} / \mathrm{L}$, microequivalents per liter; $\mu \mathrm{S} / \mathrm{cm}$, microsiemens per centimeter; $\mathrm{SC}$, specific conductance at $25^{\circ} \mathrm{C}$; $\mathrm{pH}$ values in standard units; $\mathrm{H}$, hydrogen; $\mathrm{Ca}$, calcium; $\mathrm{Mg}$, magnesium; $\mathrm{Na}$, sodium; $\mathrm{K}$, potassium; $\mathrm{NH}_{4}$, ammonium; $\mathrm{Cl}$, chloride; $\mathrm{SO}_{4}$, sulfate; $\mathrm{NO}_{3}$, nitrate; DOC, dissolved organic carbon; mg/L, milligrams per liter; $\mathrm{SWE}$, snow/water equivalent; cm, centimeters; $\delta^{34} \mathrm{~S}$, stable sulfur isotope ratio $\left({ }^{34} \mathrm{~S} /{ }^{22} \mathrm{~S}\right)$; Hg, mercury; ng/L, nanograms per liter; na, not analyzed; <, below reporting limits; NP, National Park; YNP, Yellowstone National Park; $\%$, percent]

\begin{tabular}{|c|c|c|c|c|c|c|c|c|c|c|c|c|c|c|c|c|c|c|c|c|}
\hline Site name & $\begin{array}{l}\text { Sample } \\
\text { date }\end{array}$ & $\begin{array}{l}\text { Sample } \\
\text { type }\end{array}$ & $\begin{array}{l}\text { ANC } \\
\text { ( } \mu \mathrm{eq} / \mathrm{L})\end{array}$ & $\begin{array}{c}\text { SC } \\
(\mu \mathrm{S} / \mathrm{cm})\end{array}$ & $\mathrm{pH}$ & $\begin{array}{c}H \\
(\mu e q / L)\end{array}$ & $\begin{array}{c}\mathrm{Ca} \\
(\mu \mathrm{eq} / \mathrm{L})\end{array}$ & $\underset{(\mu \mathrm{eq} / \mathrm{L})}{\mathrm{Mg}}$ & $\begin{array}{c}\mathrm{Na} \\
(\mu \mathrm{eq} / \mathrm{L})\end{array}$ & $\begin{array}{c}K \\
(\mu \mathrm{eq} / L)\end{array}$ & $\begin{array}{c}\mathrm{NH}_{4} \\
(\mu \mathrm{eq} / \mathrm{L})\end{array}$ & $\begin{array}{c}C l \\
(\mu e q / L)\end{array}$ & $\begin{array}{c}\mathrm{SO}_{4} \\
(\mu \mathrm{eq} / \mathrm{L})\end{array}$ & $\begin{array}{c}\mathrm{NO}_{3} \\
(\mu \mathrm{eq} / \mathrm{L})\end{array}$ & $\begin{array}{l}\text { DOC } \\
(\mathrm{mg} / \mathrm{L})\end{array}$ & $\begin{array}{l}\text { SWE } \\
\text { (cm) }\end{array}$ & $\begin{array}{l}\text { Snow } \\
\text { depth } \\
\text { (cm) }\end{array}$ & $\begin{array}{l}\delta^{34} S \\
\text { (per } \\
\text { mil) }\end{array}$ & $\underset{(\mathrm{ng} / \mathrm{L})}{\mathrm{Hg}}$ & $\begin{array}{c}\text { Ionic } \\
\text { balance } \\
(\%)\end{array}$ \\
\hline $\begin{array}{l}\text { Swiftcurrent 1, } \\
\text { Mont. }\end{array}$ & 3/13/2001 & E & -9.0 & 5.8 & 5.06 & 8.7 & 5.0 & $<1.7$ & 1.5 & 0.8 & 6.3 & $<1.0$ & 7.8 & 10.7 & 0.7 & 32 & 101 & na & na & 9.3 \\
\hline $\begin{array}{l}\text { Swiftcurrent 2(A), } \\
\text { Mont. }\end{array}$ & $3 / 13 / 2001$ & E & -5.1 & 6.1 & 5.07 & 8.5 & 7.0 & 2.5 & 1.7 & 0.5 & 6.9 & 1.2 & 5.7 & 7.3 & 0.6 & 43 & 132 & na & na & 31.7 \\
\hline $\begin{array}{l}\text { Swiftcurrent 2(B), } \\
\text { Mont. }\end{array}$ & $3 / 13 / 2001$ & E & -7.7 & 5.8 & 5.15 & 7.1 & 4.8 & $<1.7$ & 1.7 & 0.8 & 5.9 & $<1.0$ & 8.6 & 10.9 & 0.9 & na & 116 & na & na & 1.9 \\
\hline $\begin{array}{l}\text { Sylvan Lake (in } \\
\text { road), Wyo. }\end{array}$ & $3 / 2 / 2001$ & E & -6.9 & 5.0 & 5.10 & 7.9 & $<3.1$ & $<1.7$ & $<1.0$ & $<0.4$ & 8.7 & $<1.0$ & 7.4 & 8.3 & 0.9 & na & 60 & na & $<0.4$ & 2.8 \\
\hline $\begin{array}{l}\text { Sylvan Lake, } \\
\text { Wyo. }\end{array}$ & $3 / 2 / 2001$ & E & -6.7 & 4.0 & 5.12 & 7.6 & $<3.1$ & $<1.7$ & $<1.0$ & $<0.4$ & 7.1 & $<1.0$ & 5.0 & 8.7 & 0.4 & na & 131 & na & na & 3.5 \\
\hline $\begin{array}{l}\text { Taos Ski Valley, } \\
\text { N. Mex. }\end{array}$ & $3 / 17 / 2001$ & $\mathrm{E}$ & -6.3 & 5.9 & 5.15 & 7.1 & 7.7 & $<1.7$ & 1.7 & 1.0 & 7.3 & $<1.0$ & 10.7 & 12.8 & 0.8 & na & 260 & na & 4.0 & 2.7 \\
\hline Teton Pass, Wyo. & $3 / 19 / 2001$ & $\mathrm{E}$ & 27.6 & 8.3 & 6.17 & 0.7 & 21.0 & 10.7 & 3.9 & 3.0 & 11.8 & 3.2 & 9.4 & 12.5 & 1.4 & na & 129 & na & na & -1.6 \\
\hline $\begin{array}{l}\text { Togwotee Pass, } \\
\text { Wyo. }\end{array}$ & $3 / 12 / 2001$ & E & -4.1 & 3.2 & 5.38 & 4.2 & 4.0 & $<1.7$ & 1.0 & 0.5 & 3.5 & $<1.0$ & 3.9 & 7.1 & 0.5 & na & 122 & na & na & 8.6 \\
\hline $\begin{array}{l}\text { Twenty-one Mile, } \\
\text { Mont. }\end{array}$ & $3 / 5 / 2001$ & $\mathrm{E}$ & -6.2 & 3.8 & 5.18 & 6.6 & 3.5 & $<1.7$ & 1.1 & 0.4 & 8.2 & $<1.0$ & 4.4 & 12.4 & 0.4 & na & 110 & na & na & 8.5 \\
\hline $\begin{array}{l}\text { University Camp, } \\
\text { Colo. }\end{array}$ & $3 / 26 / 2001$ & E & -9.5 & 7.3 & 5.01 & 9.8 & 8.0 & 2.5 & 2.0 & 1.8 & 9.2 & $<1.0$ & 11.3 & 15.1 & 1.5 & na & 134 & na & na & 11.3 \\
\hline $\begin{array}{l}\text { Upper Andrews } \\
\text { Creek, Colo. }\end{array}$ & 4/25/2001 & E & 1.5 & 4.0 & 5.77 & 1.7 & 11.0 & 2.5 & 1.5 & 0.5 & 11.4 & $<1.0$ & 9.2 & 10.2 & 0.4 & na & 323 & na & na & 15.4 \\
\hline $\begin{array}{l}\text { Upper Andrews } \\
\text { Creek, Colo. }\end{array}$ & $4 / 10 / 2001$ & E & -4.2 & 5.4 & 5.27 & 5.4 & 10.5 & $<1.7$ & 2.2 & $<0.4$ & 7.2 & $<1.0$ & 8.8 & 17.0 & 0.5 & na & 170 & na & 1.5 & -1.0 \\
\hline $\begin{array}{l}\text { Upper Andrews } \\
\text { Creek, Colo. }\end{array}$ & $4 / 25 / 2001$ & $\mathrm{E}$ & -6.9 & 4.8 & 5.17 & 6.8 & 7.0 & $<1.7$ & 1.2 & 0.5 & 6.2 & $<1.0$ & 6.6 & 11.6 & 0.5 & na & 323 & na & na & 8.5 \\
\hline $\begin{array}{l}\text { W10 (Glacier } \\
\text { NP), Mont. }\end{array}$ & 3/9/2001 & E & -13.9 & 5.0 & 4.90 & 12.6 & 3.5 & $<1.7$ & 1.8 & $<0.4$ & 4.7 & $<1.0$ & 7.1 & 9.1 & 0.7 & 37 & 107 & na & 1.6 & 16.8 \\
\hline $\begin{array}{l}\text { W2 (Glacier NP), } \\
\text { Mont. }\end{array}$ & $3 / 8 / 2001$ & $\mathrm{E}$ & -14.5 & 4.8 & 5.12 & 7.6 & 4.0 & $<1.7$ & 1.9 & 1.9 & 4.9 & 1.6 & 9.9 & 10.0 & 1.0 & 30 & 92 & na & na & -2.9 \\
\hline $\begin{array}{l}\text { W5 (Glacier NP), } \\
\text { Mont. }\end{array}$ & 3/9/2001 & $\mathrm{E}$ & -8.2 & 5.6 & 5.11 & 7.8 & $<3.1$ & $<1.7$ & 1.8 & 0.8 & 5.2 & $<1.0$ & 8.1 & 10.8 & 0.7 & 34 & 103 & na & na & -9.7 \\
\hline $\begin{array}{l}\text { W6 (Glacier NP), } \\
\text { Mont. }\end{array}$ & $3 / 10 / 2001$ & E & -10.5 & 5.0 & 5.06 & 8.7 & $<3.1$ & $<1.7$ & 1.2 & $<0.4$ & 4.6 & $<1.0$ & 6.4 & 8.7 & 0.5 & 69 & 186 & na & na & -2.2 \\
\hline $\begin{array}{l}\text { W7 (Glacier NP), } \\
\text { Mont. }\end{array}$ & $3 / 10 / 2001$ & E & -11.0 & 4.4 & 5.13 & 7.4 & $<3.1$ & $<1.7$ & 2.3 & 0.9 & 3.9 & 1.7 & 6.0 & 6.9 & 0.8 & 59 & 165 & na & na & -0.4 \\
\hline
\end{tabular}


Table 10. Selected chemical and physical data for Rocky Mountain snowpack samples for 2001.—Continued

[E, depth-integrated environmental; ANC, acid neutralization capacity; $\mu$ eq/L, microequivalents per liter; $\mu \mathrm{S} / \mathrm{cm}$, microsiemens per centimeter; $\mathrm{SC}$, specific conductance at $25^{\circ} \mathrm{C} ; \mathrm{pH}$ values in standard units; $\mathrm{H}$, hydrogen; $\mathrm{Ca}$, calcium; $\mathrm{Mg}$, magnesium; $\mathrm{Na}$, sodium; $\mathrm{K}$, potassium; $\mathrm{NH}_{4}$, ammonium; $\mathrm{Cl}$, chloride; $\mathrm{SO}_{4}$, sulfate; $\mathrm{NO}_{3}$, nitrate; DOC, dissolved organic carbon; mg/L, milligrams per liter; $\mathrm{SWE}$, snow/water equivalent; cm, centimeters; $\delta^{34} \mathrm{~S}$, stable sulfur isotope ratio $\left({ }^{34} \mathrm{~S} /{ }^{22} \mathrm{~S}\right) ; \mathrm{Hg}$, mercury; ng/L, nanograms per liter; na, not analyzed; <, below reporting limits; NP, National Park; YNP, Yellowstone National Park; $\%$, percent]

\begin{tabular}{|c|c|c|c|c|c|c|c|c|c|c|c|c|c|c|c|c|c|c|c|c|}
\hline Site name & $\begin{array}{l}\text { Sample } \\
\text { date }\end{array}$ & $\begin{array}{l}\text { Sample } \\
\text { type }\end{array}$ & $\begin{array}{c}\text { ANC } \\
\text { (peq/L) }\end{array}$ & $\begin{array}{c}\text { SC } \\
(\mu \mathrm{S} / \mathrm{cm})\end{array}$ & $\mathrm{pH}$ & $\begin{array}{c}H \\
(\mu \mathrm{eq} / \mathrm{L})\end{array}$ & $\begin{array}{c}\mathrm{Ca} \\
(\mu \mathrm{eq} / \mathrm{L})\end{array}$ & $\begin{array}{c}\mathrm{Mg} \\
(\mu \mathrm{eq} / \mathrm{L})\end{array}$ & $\begin{array}{c}\mathrm{Na} \\
(\mu \mathrm{eq} / \mathrm{L})\end{array}$ & $\begin{array}{c}K \\
(\mu \mathrm{eq} / L)\end{array}$ & $\begin{array}{c}\mathrm{NH}_{4} \\
(\mu \mathrm{eq} / \mathrm{L})\end{array}$ & $\begin{array}{c}\text { CI } \\
(\mu \mathrm{eq} / \mathrm{L})\end{array}$ & $\begin{array}{c}\mathrm{SO}_{4} \\
(\mu \mathrm{eq} / \mathrm{L})\end{array}$ & $\begin{array}{c}\mathrm{NO}_{3} \\
(\mu \mathrm{eq} / \mathrm{L})\end{array}$ & $\begin{array}{c}\text { DOC } \\
\text { (mg/L) }\end{array}$ & $\begin{array}{l}\text { SWE } \\
\text { (cm) }\end{array}$ & $\begin{array}{l}\text { Snow } \\
\text { depth } \\
\text { (cm) }\end{array}$ & $\begin{array}{l}\delta^{34} S \\
\text { (per } \\
\text { mil) }\end{array}$ & $\underset{(\mathrm{ng} / \mathrm{L})}{\mathrm{Hg}}$ & $\begin{array}{c}\text { lonic } \\
\text { balance } \\
(\%)\end{array}$ \\
\hline $\begin{array}{l}\text { W8 (Glacier NP), } \\
\text { Mont. }\end{array}$ & $3 / 10 / 2001$ & E & -15.3 & 4.5 & 5.07 & 8.5 & $<3.1$ & $<1.7$ & 1.6 & $<0.4$ & 3.5 & $<1.0$ & 6.3 & 7.9 & 0.7 & 49 & 136 & na & na & -1.8 \\
\hline $\begin{array}{l}\text { W9 (Glacier NP), } \\
\text { Mont. }\end{array}$ & 3/9/2001 & E & 0.2 & 4.6 & 5.11 & 7.8 & 4.0 & $<1.7$ & 2.2 & 1.1 & 3.1 & 2.0 & 6.7 & 8.6 & 0.8 & 42 & 125 & na & na & 2.2 \\
\hline $\begin{array}{l}\text { West Yellowstone, } \\
\text { Mont. }\end{array}$ & $3 / 5 / 2001$ & E & -9.8 & 6.5 & 5.06 & 8.7 & 4.5 & $<1.7$ & 1.4 & 0.6 & 13.6 & 1.1 & 7.6 & 18.6 & 0.9 & na & 55 & na & 1.8 & 2.5 \\
\hline $\begin{array}{l}\text { Wolf Creek Pass, } \\
\text { Colo. }\end{array}$ & $3 / 27 / 2001$ & E & -5.9 & 5.2 & 5.17 & 6.8 & 7.5 & $<1.7$ & 1.4 & 2.0 & 5.3 & $<1.0$ & 9.0 & 11.1 & 0.9 & na & 240 & na & 5.8 & 6.8 \\
\hline $\begin{array}{l}\text { Zirkels South } \\
\text { Boundary, } \\
\text { Colo. }\end{array}$ & 4/4/2001 & E & -13.2 & 5.6 & 4.97 & 10.7 & 7.5 & $<1.7$ & 2.8 & 0.4 & 6.7 & $<1.0$ & 11.0 & 13.0 & 0.5 & na & 277 & 6.2 & 0.9 & 8.0 \\
\hline
\end{tabular}


[E, depth-integrated environmental; ANC, acid neutralization capacity; $\mu \mathrm{eq} / \mathrm{L}$, microequivalents per liter; $\mu \mathrm{S} / \mathrm{cm}$, microsiemens per centimeter; $\mathrm{SC}$, specific conductance at $25^{\circ} \mathrm{C}$; $\mathrm{pH}$ values in standard units; $\mathrm{H}$, hydrogen; $\mathrm{Ca}$, calcium; $\mathrm{Mg}$, magnesium; $\mathrm{Na}$, sodium; $\mathrm{K}$, potassium; $\mathrm{NH}_{4}$, ammonium; $\mathrm{Cl}$, chloride; $\mathrm{SO}_{4}$, sulfate; $\mathrm{NO}_{3}$, nitrate; DOC, dissolved organic carbon; mg/L, milligrams per liter; $\mathrm{SWE}$, snow/ water equivalent; cm, centimeters; $\delta^{34} \mathrm{~S}$, stable sulfur isotope ratio $\left({ }^{34} \mathrm{~S} /{ }^{22} \mathrm{~S}\right) ; \mathrm{Hg}$, mercury; ng/L, nanograms per liter; na, not analyzed; <, below reporting limits; NP, National Park; (), suspect ionic balance; $\%$, percent]

\begin{tabular}{|c|c|c|c|c|c|c|c|c|c|c|c|c|c|c|c|c|c|c|c|c|}
\hline Site name & $\begin{array}{l}\text { Sample } \\
\text { date }\end{array}$ & $\begin{array}{c}\text { Sample } \\
\text { type }\end{array}$ & $\begin{array}{c}\text { ANC } \\
\text { ( } \mu \mathrm{eq} / \mathrm{L})\end{array}$ & $\begin{array}{c}\mathrm{SC} \\
(\mu \mathrm{S} / \mathrm{cm})\end{array}$ & $\mathrm{pH}$ & $\begin{array}{c}H \\
(\mu \mathrm{eq} / L)\end{array}$ & $\begin{array}{c}\text { Ca } \\
(\mu \mathrm{eq} / \mathrm{L})\end{array}$ & $\begin{array}{c}\mathrm{Mg} \\
(\mu \mathrm{eq} / \mathrm{L})\end{array}$ & $\underset{(\mu \mathrm{eq} / \mathrm{L})}{\mathrm{Na}}$ & $\begin{array}{c}K \\
(\mu e q / L)\end{array}$ & $\begin{array}{c}\mathrm{NH}_{4} \\
(\mu \mathrm{eq} / \mathrm{L})\end{array}$ & $\begin{array}{c}\text { CI } \\
(\mu \mathrm{eq} / \mathrm{L})\end{array}$ & $\begin{array}{c}\mathrm{SO}_{4} \\
(\mu \mathrm{eq} / \mathrm{L})\end{array}$ & $\begin{array}{c}\mathrm{NO}_{3} \\
(\mu \mathrm{eq} / \mathrm{L})\end{array}$ & $\begin{array}{l}\text { DOC } \\
\text { (mg/L) }\end{array}$ & $\begin{array}{l}\text { SWE } \\
\text { (cm) }\end{array}$ & $\begin{array}{l}\text { Snow } \\
\text { depth } \\
\text { (cm) }\end{array}$ & $\begin{array}{c}\delta^{34} \mathbf{S} \\
\text { (per mil) }\end{array}$ & $\begin{array}{c}\mathrm{Hg} \\
\text { (ng/L) }\end{array}$ & $\begin{array}{c}\text { Ionic } \\
\text { balance } \\
(\%)\end{array}$ \\
\hline $\begin{array}{l}\text { Andrews But- } \\
\text { tress, Colo. }\end{array}$ & 4/1/2002 & E & -9.8 & 6.2 & 5.10 & 7.9 & 10.5 & 2.5 & 2.1 & 0.9 & 4.1 & 1.6 & 5.5 & 17.1 & 0.5 & 127 & 327 & na & 1.5 & 7.2 \\
\hline $\begin{array}{l}\text { Apgar Lookout, } \\
\text { Mont. }\end{array}$ & $3 / 4 / 2002$ & $\mathrm{E}$ & -9.7 & 4.2 & 5.22 & 6.0 & $<3.1$ & $<1.7$ & $<1.0$ & $<0.4$ & 5.5 & $<1.0$ & 3.4 & 6.8 & 0.5 & 50 & 143 & na & 1.0 & 5.8 \\
\hline $\begin{array}{l}\text { Arapaho Creek, } \\
\text { Colo. }\end{array}$ & 3/8/2002 & E & -18.5 & 8.8 & 4.82 & 15.1 & 7.5 & $<1.7$ & 2.4 & 0.7 & 2.2 & 1.3 & 5.0 & 21.2 & 0.6 & 21 & 89 & na & 1.4 & 0.7 \\
\hline $\begin{array}{l}\text { Banner Summit, } \\
\text { Idaho }\end{array}$ & $3 / 15 / 2002$ & E & -3.6 & 2.9 & 5.48 & 3.3 & $<3.1$ & $<1.7$ & 1.4 & $<0.4$ & 3.6 & $<1.0$ & 2.5 & 3.4 & $<0.4$ & 63 & 201 & 6.5 & 1.5 & 16.4 \\
\hline Bear Lake, Colo. & 4/3/2002 & E & -5.1 & 5.7 & 5.36 & 4.4 & 13.5 & 2.5 & 1.9 & 1.4 & 7.4 & 1.3 & 8.2 & 16.1 & 0.8 & 23 & 95 & na & 3.2 & 9.5 \\
\hline $\begin{array}{l}\text { Berthoud Pass, } \\
\text { Colo. }\end{array}$ & 4/4/2002 & E & -6.3 & 5.2 & 5.23 & 5.9 & 10.5 & $<1.7$ & 2.4 & 0.8 & 3.7 & 2.8 & 4.9 & 13.4 & 0.6 & 37 & 130 & na & 3.1 & 4.7 \\
\hline $\begin{array}{l}\text { Big Mountain, } \\
\text { Mont. }\end{array}$ & $3 / 10 / 2002$ & $\mathrm{E}$ & -7.5 & 3.5 & 5.29 & 5.1 & $<3.1$ & $<1.7$ & 5.4 & $<0.4$ & 4.9 & $<1.0$ & 2.6 & 4.9 & $<0.4$ & 99 & 298 & 5.9 & 1.1 & 34.1 \\
\hline Big Sky, Mont. & $3 / 17 / 2002$ & $\mathrm{E}$ & -2.4 & 3.0 & 5.54 & 2.9 & 5.0 & $<1.7$ & $<1.0$ & 0.5 & 4.5 & $<1.0$ & 2.9 & 4.7 & 0.6 & 38 & 120 & na & 2.0 & 25.7 \\
\hline $\begin{array}{l}\text { Brooklyn Lake, } \\
\text { Wyo. }\end{array}$ & 3/19/2002 & E & 10.1 & 6.1 & 6.00 & 1.0 & 24.5 & 4.9 & 5.5 & 2.3 & 4.3 & 1.5 & 11.9 & 13.5 & 1.5 & 41 & 149 & 6.6 & 5.9 & 6.9 \\
\hline Brumley, Colo. & $3 / 25 / 2002$ & E & 21.5 & 5.7 & 6.37 & 0.4 & 31.9 & 9.9 & 1.2 & 7.2 & $<1.0$ & 1.8 & 5.9 & 10.5 & 3.4 & 21 & 67 & na & 6.4 & 12.0 \\
\hline $\begin{array}{l}\text { Buffalo Pass, } \\
\text { Colo. }\end{array}$ & $4 / 4 / 2002$ & E & -8.9 & 6.3 & 5.17 & 6.8 & 11.5 & 2.5 & 1.8 & 0.7 & 5.8 & 1.2 & 9.3 & 15.2 & 0.5 & 81 & 260 & 7.0 & 2.0 & 5.9 \\
\hline $\begin{array}{l}\text { Cameron Pass, } \\
\text { Colo. }\end{array}$ & 4/4/2002 & E & -6.1 & 5.3 & 5.29 & 5.1 & 11.0 & 2.5 & 3.6 & 0.8 & 4.0 & 1.7 & 8.0 & 12.7 & 0.6 & 36 & 122 & na & 2.6 & 9.4 \\
\hline Canyon, Wyo. & $2 / 27 / 2002$ & E & -7.8 & 3.5 & 5.34 & 4.6 & $<3.1$ & $<1.7$ & 1.3 & $<0.4$ & 6.7 & 1.2 & 3.1 & 7.3 & $<0.4$ & 27 & 108 & na & 0.7 & 4.1 \\
\hline $\begin{array}{l}\text { Chief Joseph } \\
\text { Pass, Mont. }\end{array}$ & 3/7/2002 & E & -4.1 & 2.8 & 5.44 & 3.6 & $<3.1$ & $<1.7$ & $<1.0$ & $<0.4$ & 3.5 & $<1.0$ & 2.1 & 3.8 & $<0.4$ & 40 & 145 & 6.3 & 1.4 & 9.7 \\
\hline Culebra, Colo. & $3 / 20 / 2002$ & $\mathrm{E}$ & 6.0 & 5.7 & 6.00 & 1.0 & 21.5 & 2.5 & 2.9 & 2.2 & 6.3 & 1.5 & 8.1 & 15.9 & 0.7 & 24 & 103 & 5.2 & 3.9 & 6.9 \\
\hline $\begin{array}{l}\text { Daisy Pass, } \\
\text { Mont. }\end{array}$ & $3 / 5 / 2002$ & E & -4.0 & 3.7 & 5.43 & 3.7 & 3.5 & $<1.7$ & $<1.0$ & 0.7 & 4.7 & $<1.0$ & 3.5 & 6.0 & 0.4 & 65 & 207 & na & 1.6 & 14.4 \\
\hline $\begin{array}{l}\text { Deadman Pass, } \\
\text { Colo. }\end{array}$ & $3 / 19 / 2002$ & E & -6.6 & 5.7 & 5.38 & 4.2 & 14.0 & 2.5 & 2.8 & 1.4 & 6.6 & 1.4 & 9.5 & 16.0 & 1.0 & 36 & 130 & na & 5.8 & 7.7 \\
\hline $\begin{array}{l}\text { Divide Peak, } \\
\text { Wyo. }\end{array}$ & $3 / 20 / 2002$ & $\mathrm{E}$ & -1.3 & 6.1 & 5.51 & 3.1 & 15.5 & 3.3 & 4.9 & 0.5 & 6.3 & 1.8 & 9.7 & 17.0 & 0.7 & 34 & 126 & na & 4.5 & 8.2 \\
\hline Dry Lake, Colo. & $3 / 13 / 2002$ & $\mathrm{E}$ & -16.8 & 9.1 & 4.85 & 14.1 & 9.0 & 2.5 & 3.2 & 0.7 & 6.4 & 2.3 & 8.8 & 21.5 & $<0.4$ & 43 & 143 & na & 0.9 & 4.8 \\
\hline $\begin{array}{l}\text { Dunckley Pass, } \\
\text { Colo. }\end{array}$ & 4/3/2002 & E & -2.1 & 5.4 & 5.58 & 2.6 & 13.0 & 2.5 & 1.2 & 1.9 & 3.8 & 1.1 & 6.9 & 14.1 & 0.7 & 35 & 135 & na & 4.0 & 6.3 \\
\hline
\end{tabular}


Table 11. Selected chemical and physical data for Rocky Mountain snowpack samples for 2002.—Continued

[[E, depth-integrated environmental; ANC, acid neutralization capacity; $\mu \mathrm{eq} / \mathrm{L}$, microequivalents per liter; $\mu \mathrm{S} / \mathrm{cm}$, microsiemens per centimeter; $\mathrm{SC}$, specific conductance at $25^{\circ} \mathrm{C}$; $\mathrm{pH}$ values in standard units; $\mathrm{H}$, hydrogen; Ca, calcium; Mg, magnesium; $\mathrm{Na}$, sodium; $\mathrm{K}$, potassium; $\mathrm{NH}_{4}$, ammonium; $\mathrm{Cl}$, chloride; $\mathrm{SO}_{4}$, sulfate; $\mathrm{NO}_{3}$, nitrate; DOC, dissolved organic carbon; mg/L, milligrams per liter; $\mathrm{SWE}$, snow/ water equivalent; cm, centimeters; $\delta^{34} \mathrm{~S}$, stable sulfur isotope ratio $\left({ }^{34} \mathrm{~S} /{ }^{2} \mathrm{~S}\right) ; \mathrm{Hg}$, mercury; ng/L, nanograms per liter; na, not analyzed; <, below reporting limits; NP, National Park; (), suspect ionic balance; $\%$, percent]

\begin{tabular}{|c|c|c|c|c|c|c|c|c|c|c|c|c|c|c|c|c|c|c|c|c|}
\hline Site name & $\begin{array}{l}\text { Sample } \\
\text { date }\end{array}$ & $\begin{array}{l}\text { Sample } \\
\text { type }\end{array}$ & $\begin{array}{c}\text { ANC } \\
\text { ( } \mu \mathrm{eq} / \mathrm{L})\end{array}$ & $\begin{array}{c}\text { SC } \\
(\mu \mathrm{S} / \mathrm{cm})\end{array}$ & $\mathrm{pH}$ & $\underset{\text { (peq/L) }}{H}$ & 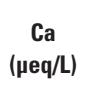 & $\underset{(\mu \mathrm{gq} / \mathrm{L})}{\mathrm{Mg}}$ & $\begin{array}{c}\mathrm{Na} \\
(\mu \mathrm{eq} / \mathrm{L})\end{array}$ & 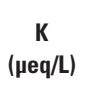 & $\begin{array}{c}\mathrm{NH}_{4} \\
(\mu \mathrm{eq} / \mathrm{L})\end{array}$ & 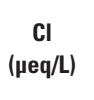 & $\begin{array}{c}\mathrm{SO}_{4} \\
(\mu \mathrm{eq} / \mathrm{L})\end{array}$ & 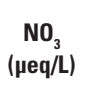 & $\begin{array}{l}\text { DOC } \\
(\mathrm{mg} / \mathrm{L})\end{array}$ & $\begin{array}{l}\text { SWE } \\
(\mathrm{cm})\end{array}$ & $\begin{array}{l}\text { Snow } \\
\text { depth } \\
\text { (cm) }\end{array}$ & $\begin{array}{c}\delta^{34} \mathrm{~S} \\
\text { (per mil) }\end{array}$ & $\underset{(\mathrm{ng} / \mathrm{L})}{\mathrm{Hg}}$ & $\begin{array}{c}\text { Ionic } \\
\text { balance } \\
(\%)\end{array}$ \\
\hline $\begin{array}{l}\text { E3 (Glacier NP), } \\
\text { Mont. }\end{array}$ & $3 / 12 / 2002$ & E & -8.4 & 3.7 & 5.23 & 5.9 & $<3.1$ & $<1.7$ & 1.1 & 0.7 & 3.9 & $<1.0$ & 3.6 & 6.9 & $<0.4$ & 55 & 184 & na & 2.1 & 5.7 \\
\hline $\begin{array}{l}\text { E5 (Glacier NP), } \\
\text { Mont. }\end{array}$ & $3 / 13 / 2002$ & E & -6.2 & 3.0 & 5.27 & 5.4 & $<3.1$ & $<1.7$ & $<1.0$ & $<0.4$ & 2.4 & $<1.0$ & 2.2 & 4.2 & $<0.4$ & 113 & 333 & na & 1.4 & 9.8 \\
\hline $\begin{array}{l}\text { E6 (Glacier NP), } \\
\text { Mont. }\end{array}$ & $3 / 13 / 2002$ & $\mathrm{E}$ & -7.0 & 3.3 & 5.27 & 5.4 & $<3.1$ & $<1.7$ & $<1.0$ & 0.6 & 2.2 & $<1.0$ & 2.5 & 4.5 & $<0.4$ & 88 & 280 & na & 7.8 & 7.3 \\
\hline Elk River, Colo. & $3 / 12 / 2002$ & E & -7.3 & 6.1 & 5.27 & 5.4 & 12.5 & 2.5 & 6.6 & 0.5 & 5.6 & 2.4 & 9.9 & 16.2 & $<0.4$ & 31 & 122 & na & 1.5 & 7.4 \\
\hline $\begin{array}{l}\text { Elkhart Park, } \\
\text { Wyo. }\end{array}$ & $3 / 26 / 2002$ & E & -8.3 & 5.0 & 5.27 & 5.4 & 6.0 & $<1.7$ & 1.7 & 0.8 & 5.5 & 1.2 & 5.2 & 7.5 & 0.5 & 33 & 120 & 7.6 & 1.1 & 16.2 \\
\hline $\begin{array}{c}\text { Forest Canyon } \\
\text { Pass, Colo. }\end{array}$ & $3 / 29 / 2002$ & $\mathrm{E}$ & -6.4 & 4.7 & 5.23 & 5.9 & 9.5 & $<1.7$ & $<1.0$ & 0.9 & 2.9 & 1.2 & 5.9 & 12.3 & 0.8 & 56 & 200 & na & 6.3 & -0.5 \\
\hline $\begin{array}{l}\text { Four Mile } \\
\text { Meadow, } \\
\text { Wyo. }\end{array}$ & $3 / 27 / 2002$ & $\mathrm{E}$ & -4.6 & 4.5 & 5.49 & 3.2 & 7.0 & $<1.7$ & 2.9 & 1.1 & 8.5 & 1.5 & 5.0 & 10.0 & 0.8 & 20 & 84 & 6.9 & 1.2 & 15.6 \\
\hline $\begin{array}{l}\text { Fremont Pass, } \\
\text { Colo. }\end{array}$ & $4 / 5 / 2002$ & E & -1.9 & 4.5 & 5.58 & 2.6 & 10.5 & $<1.7$ & $<1.0$ & 1.5 & 1.9 & 1.1 & 3.3 & 9.9 & 0.7 & 29 & 122 & na & 3.0 & 7.3 \\
\hline $\begin{array}{l}\text { Galena Summit, } \\
\text { Idaho }\end{array}$ & $3 / 15 / 2002$ & E & -4.6 & 3.0 & 5.38 & 4.2 & $<3.1$ & $<1.7$ & 8.2 & $<0.4$ & 1.3 & 5.7 & 2.7 & 3.8 & 0.5 & 37 & 135 & 6.1 & 0.4 & 5.8 \\
\hline $\begin{array}{l}\text { Garnet Canyon, } \\
\text { Wyo. }\end{array}$ & $4 / 4 / 2002$ & E & -4.9 & 4.3 & 5.39 & 4.1 & 5.5 & $<1.7$ & 1.6 & 1.0 & 6.2 & 1.5 & 6.9 & 8.5 & 0.4 & 51 & 137 & na & 3.4 & 4.0 \\
\hline $\begin{array}{l}\text { Grand Mesa, } \\
\text { Colo. }\end{array}$ & $3 / 22 / 2002$ & E & 25.5 & 6.8 & 6.40 & 0.4 & 34.4 & 5.8 & 2.0 & 3.8 & 3.7 & 1.9 & 7.4 & 15.8 & 1.6 & 30 & 102 & na & 9.4 & -0.5 \\
\hline $\begin{array}{l}\text { Granite Park, } \\
\text { Mont. }\end{array}$ & $3 / 13 / 2002$ & E & -7.0 & 3.6 & 5.25 & 5.6 & $<3.1$ & $<1.7$ & $<1.0$ & $<0.4$ & 3.4 & $<1.0$ & 2.6 & 4.8 & $<0.4$ & 134 & 370 & na & 0.6 & 9.7 \\
\hline $\begin{array}{l}\text { Granite Pass, } \\
\text { Mont. }\end{array}$ & $3 / 7 / 2002$ & E & -3.2 & 2.9 & 5.49 & 3.2 & $<3.1$ & $<1.7$ & $<1.0$ & 0.6 & 1.4 & $<1.0$ & 1.9 & 2.8 & 0.4 & 75 & 240 & na & 11.1 & 5.3 \\
\hline $\begin{array}{l}\text { Green Ridge, } \\
\text { Colo. }\end{array}$ & $3 / 7 / 2002$ & E & 32.7 & 9.0 & 6.64 & 0.2 & 41.9 & 14.8 & 2.8 & 6.9 & 4.6 & 1.8 & 8.3 & 23.2 & 0.7 & 14 & 59 & na & 1.8 & 3.8 \\
\hline $\begin{array}{l}\text { Gypsum Creek, } \\
\text { Wyo. }\end{array}$ & $3 / 26 / 2002$ & E & -8.6 & 5.1 & 5.29 & 5.1 & 6.5 & $<1.7$ & 3.1 & 0.5 & 7.3 & 1.5 & 6.8 & 10.9 & $<0.4$ & 29 & 105 & na & 1.0 & 7.9 \\
\hline $\begin{array}{l}\text { Halfmoon Creek, } \\
\text { Colo. }\end{array}$ & $4 / 5 / 2002$ & E & 2.9 & 5.6 & 5.78 & 1.7 & 20.0 & 4.1 & 1.7 & 2.0 & 3.3 & 1.6 & 6.9 & 15.1 & 1.9 & 12 & 53 & na & 3.6 & 10.6 \\
\hline $\begin{array}{l}\text { Hopewell, } \\
\text { N. Mex. }\end{array}$ & $3 / 15 / 2002$ & E & 2.8 & 5.7 & 5.81 & 1.5 & 24.5 & 4.1 & 3.0 & 1.5 & 5.0 & 2.4 & 8.4 & 17.5 & 0.5 & 22 & 88 & 5.4 & 3.6 & 12.0 \\
\hline Kings Hill, Mont. & $3 / 2 / 2002$ & $\mathrm{E}$ & -1.7 & 3.9 & 5.60 & 2.5 & 10.0 & $<1.7$ & $<1.0$ & 1.5 & 7.8 & $<1.0$ & 4.5 & 8.9 & 0.8 & 33 & 113 & na & 5.0 & 24.0 \\
\hline
\end{tabular}


[[E, depth-integrated environmental; ANC, acid neutralization capacity; $\mu \mathrm{eq} / \mathrm{L}$, microequivalents per liter; $\mu \mathrm{S} / \mathrm{cm}$, microsiemens per centimeter; $\mathrm{SC}$, specific conductance at $25^{\circ} \mathrm{C}$; $\mathrm{pH}$ values in standard units; $\mathrm{H}$, hydrogen; Ca, calcium; $\mathrm{Mg}$, magnesium; $\mathrm{Na}$, sodium; $\mathrm{K}$, potassium; $\mathrm{NH}_{4}$, ammonium; $\mathrm{Cl}$, chloride; $\mathrm{SO}_{4}$, sulfate; $\mathrm{NO}_{3}$, nitrate; DOC, dissolved organic carbon; mg/L, milligrams per liter; $\mathrm{SWE}$, snow/ water equivalent; cm, centimeters; $\delta^{34} \mathrm{~S}$, stable sulfur isotope ratio $\left({ }^{34} \mathrm{~S} /{ }^{32} \mathrm{~S}\right) ; \mathrm{Hg}$, mercury; ng/L, nanograms per liter; na, not analyzed; <, below reporting limits; NP, National Park; (), suspect ionic balance; $\%$, percent]

\begin{tabular}{|c|c|c|c|c|c|c|c|c|c|c|c|c|c|c|c|c|c|c|c|c|}
\hline Site name & $\begin{array}{l}\text { Sample } \\
\text { date }\end{array}$ & $\begin{array}{l}\text { Sample } \\
\text { type }\end{array}$ & $\begin{array}{c}\text { ANC } \\
(\mu \mathrm{eq} / \mathrm{L})\end{array}$ & $\begin{array}{c}\text { SC } \\
(\mu \mathrm{S} / \mathrm{cm})\end{array}$ & $\mathrm{pH}$ & $\underset{(\mu e q / L)}{H}$ & $\begin{array}{c}\mathrm{Ca} \\
(\mu \mathrm{eq} / \mathrm{L})\end{array}$ & $\underset{(\mu \mathrm{gg} / \mathrm{L})}{\mathrm{Mg}}$ & $\begin{array}{c}\mathrm{Na} \\
(\mu \mathrm{eq} / \mathrm{L})\end{array}$ & $\begin{array}{c}K \\
(\mu e q / L)\end{array}$ & 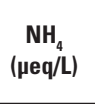 & 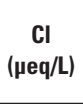 & $\begin{array}{c}\mathrm{SO}_{4} \\
(\mu \mathrm{eq} / \mathrm{L})\end{array}$ & $\begin{array}{c}\mathrm{NO}_{3} \\
(\mu \mathrm{eq} / \mathrm{L})\end{array}$ & $\begin{array}{l}\text { DOC } \\
\text { (mg/L) }\end{array}$ & $\begin{array}{l}\text { SWE } \\
(\mathrm{cm})\end{array}$ & $\begin{array}{l}\text { Snow } \\
\text { depth } \\
\text { (cm) }\end{array}$ & $\begin{array}{c}\delta^{34} S \\
\text { (per mil) }\end{array}$ & $\begin{array}{c}\mathrm{Hg} \\
\text { (ng/L) }\end{array}$ & $\begin{array}{c}\text { lonic } \\
\text { balance } \\
(\%)\end{array}$ \\
\hline $\begin{array}{l}\text { Lake Irene For- } \\
\text { est, Colo. }\end{array}$ & $3 / 29 / 2002$ & E & -5.4 & 5.1 & 5.42 & 3.8 & 15.0 & 2.5 & 1.6 & 1.1 & 4.7 & 1.2 & 7.0 & 14.3 & 0.6 & 35 & 132 & na & 3.0 & 12.1 \\
\hline $\begin{array}{l}\text { Lake Irene Mead- } \\
\text { ow, Colo. }\end{array}$ & $3 / 29 / 2002$ & E & -8.7 & 5.4 & 5.14 & 7.2 & 10.5 & $<1.7$ & 1.3 & 0.6 & 3.5 & 1.1 & 5.8 & 14.7 & $<0.4$ & 43 & 135 & na & 1.3 & 3.4 \\
\hline $\begin{array}{l}\text { Lewis Lake Di- } \\
\text { vide, Wyo. }\end{array}$ & $3 / 1 / 2002$ & E & -1.7 & 3.9 & 5.53 & 3.0 & 4.0 & $<1.7$ & 1.6 & $<0.4$ & 5.7 & $<1.0$ & 4.3 & 6.0 & $<0.4$ & 66 & 209 & na & 1.2 & 16.1 \\
\hline Lionshead, Mont. & $3 / 4 / 2002$ & E & -3.4 & 4.9 & 5.40 & 4.0 & 3.5 & $<1.7$ & $<1.0$ & 1.1 & 13.9 & $<1.0$ & 5.9 & 10.8 & 0.4 & 46 & 149 & na & 8.2 & 14.7 \\
\hline $\begin{array}{l}\text { Lizard Head } \\
\text { Pass, Colo. }\end{array}$ & $3 / 21 / 2002$ & E & 51.5 & 7.7 & 6.74 & 0.2 & 41.4 & 3.3 & 2.2 & 2.4 & 3.8 & 1.5 & 5.6 & 13.5 & 0.7 & 26 & 106 & 4.3 & 3.8 & $(-15.1)$ \\
\hline $\begin{array}{l}\text { Loch Vale Forest, } \\
\text { Colo. }\end{array}$ & 4/1/2002 & E & -7.1 & 7.0 & 5.21 & 6.2 & 13.5 & 2.5 & 2.1 & 1.0 & 5.7 & 1.6 & 7.8 & 19.3 & 0.7 & 51 & 153 & na & 3.8 & 3.7 \\
\hline $\begin{array}{l}\text { Loch Vale } \\
\text { Meadow, } \\
\text { Colo. }\end{array}$ & $4 / 1 / 2002$ & $\mathrm{E}$ & -6.4 & 3.6 & 5.22 & 6.0 & $<3.1$ & $<1.7$ & $<1.0$ & $<0.4$ & 3.1 & $<1.0$ & 1.6 & 8.2 & $<0.4$ & 37 & 110 & na & 2.5 & -3.5 \\
\hline $\begin{array}{l}\text { Loveland Pass, } \\
\text { Colo. }\end{array}$ & $4 / 6 / 2002$ & E & 0.0 & 7.7 & 5.72 & 1.9 & 25.0 & 7.4 & 4.8 & 0.7 & 6.1 & 7.0 & 7.4 & 24.5 & 0.7 & 47 & 130 & na & 2.8 & 8.3 \\
\hline Lynx Pass, Colo. & $3 / 13 / 2002$ & E & -11.8 & 6.4 & 5.04 & 9.1 & 10.0 & $<1.7$ & 1.6 & 0.5 & 3.5 & 1.0 & 5.6 & 16.7 & 0.4 & 28 & 108 & na & 1.0 & 2.9 \\
\hline $\begin{array}{l}\text { Molas Lake, } \\
\text { Colo. }\end{array}$ & $3 / 27 / 2002$ & E & 15.8 & 5.4 & 6.33 & 0.5 & 25.4 & 2.5 & 3.7 & 1.3 & 2.6 & 3.6 & 4.3 & 10.8 & $<0.4$ & 25 & 89 & 4.7 & 3.6 & 2.0 \\
\hline $\begin{array}{l}\text { Monarch Pass, } \\
\text { Colo. }\end{array}$ & $3 / 25 / 2002$ & E & 30.4 & 9.2 & 6.47 & 0.3 & 51.4 & 6.6 & 2.9 & 3.9 & 5.7 & 3.1 & 8.2 & 22.9 & 1.8 & 20 & 67 & 5.8 & 9.6 & 4.6 \\
\hline $\begin{array}{l}\text { Monida Pass, } \\
\text { Mont. }\end{array}$ & $2 / 28 / 2002$ & E & -4.4 & 5.3 & 5.55 & 2.8 & 11.0 & 4.1 & 1.5 & 0.7 & 12.6 & 1.9 & 5.5 & 17.4 & 0.6 & 5 & 20 & na & 0.6 & 13.7 \\
\hline $\begin{array}{l}\text { Mount Belmont, } \\
\text { Mont. }\end{array}$ & $3 / 1 / 2002$ & E & -8.2 & 5.3 & 5.15 & 7.1 & 5.0 & $<1.7$ & $<1.0$ & 4.3 & 6.5 & 1.0 & 5.1 & 9.5 & 1.4 & 18 & 63 & na & 1.7 & 18.9 \\
\hline $\begin{array}{l}\text { Niwot Snotel, } \\
\text { Colo. }\end{array}$ & 4/2/2002 & E & -5.9 & 7.3 & 5.22 & 6.0 & 15.0 & 3.3 & 1.9 & 2.5 & 10.9 & 1.7 & 9.7 & 20.5 & 1.8 & 18 & 71 & na & 3.3 & 10.7 \\
\hline $\begin{array}{l}\text { Noisy Basin, } \\
\text { Mont. }\end{array}$ & $3 / 8 / 2002$ & E & -5.9 & 3.9 & 5.24 & 5.8 & $<3.1$ & $<1.7$ & $<1.0$ & 0.7 & 5.0 & $<1.0$ & 3.2 & 6.2 & 0.4 & 83 & 275 & na & 2.3 & 10.2 \\
\hline Old Battle, Wyo. & $3 / 20 / 2002$ & E & -5.3 & 4.9 & 5.25 & 5.6 & 9.0 & $<1.7$ & 2.8 & $<0.4$ & 5.2 & 1.2 & 7.4 & 13.1 & $<0.4$ & 61 & 202 & na & 2.3 & 1.9 \\
\hline $\begin{array}{l}\text { Old Faithful Fire } \\
\text { Road, Wyo. }\end{array}$ & $2 / 27 / 2002$ & E & -4.5 & 4.6 & 5.41 & 3.9 & 4.5 & $<1.7$ & 2.1 & 0.7 & 8.0 & 1.3 & 6.0 & 9.1 & $<0.4$ & 22 & 88 & na & 0.9 & 7.9 \\
\hline $\begin{array}{l}\text { Old Faithful (in } \\
\text { road), Wyo. }\end{array}$ & $2 / 27 / 2002$ & E & -1.4 & 8.2 & 5.44 & 3.6 & na & na & 7.5 & 4.2 & 15.0 & 10.6 & 13.4 & 10.3 & 3.3 & na & 42 & na & 4.2 & -6.2 \\
\hline $\begin{array}{l}\text { Phantom Valley, } \\
\text { Colo. }\end{array}$ & 3/7/2002 & E & -15.9 & 7.8 & 4.90 & 12.6 & 7.5 & $<1.7$ & 1.8 & 0.7 & 5.2 & 1.1 & 7.4 & 19.0 & 0.4 & 16 & 70 & na & 1.1 & 0.2 \\
\hline
\end{tabular}


Table 11. Selected chemical and physical data for Rocky Mountain snowpack samples for 2002.—Continued

[E, depth-integrated environmental; ANC, acid neutralization capacity; $\mu \mathrm{eq} / \mathrm{L}$, microequivalents per liter; $\mu \mathrm{S} / \mathrm{cm}$, microsiemens per centimeter; $\mathrm{SC}$, specific conductance at $25^{\circ} \mathrm{C} ; \mathrm{pH}$ values in standard units; $\mathrm{H}$, hydrogen; Ca, calcium; Mg, magnesium; $\mathrm{Na}$, sodium; $\mathrm{K}$, potassium; $\mathrm{NH}_{4}$, ammonium; $\mathrm{Cl}$, chloride; $\mathrm{SO}_{4}$, sulfate; $\mathrm{NO}_{3}$, nitrate; DOC, dissolved organic carbon; mg/L, milligrams per liter; $\mathrm{SWE}$, snow/ water equivalent; cm, centimeters; $\delta^{34} \mathrm{~S}$, stable sulfur isotope ratio $\left({ }^{34} \mathrm{~S} /{ }^{22} \mathrm{~S}\right) ; \mathrm{Hg}$, mercury; ng/L, nanograms per liter; na, not analyzed; <, below reporting limits; NP, National Park; (), suspect ionic balance; $\%$, percent]

\begin{tabular}{|c|c|c|c|c|c|c|c|c|c|c|c|c|c|c|c|c|c|c|c|c|}
\hline Site name & $\begin{array}{l}\text { Sample } \\
\text { date }\end{array}$ & $\begin{array}{l}\text { Sample } \\
\text { type }\end{array}$ & $\begin{array}{c}\text { ANC } \\
\text { ( } \mu \mathrm{eq} / \mathrm{L})\end{array}$ & $\begin{array}{c}\mathrm{SC} \\
(\mu \mathrm{S} / \mathrm{cm})\end{array}$ & $\mathrm{pH}$ & $\begin{array}{c}H \\
(\mu e q / L)\end{array}$ & $\begin{array}{c}\text { Ca } \\
(\mu e q / L)\end{array}$ & $\underset{(\mu \mathrm{eq} / \mathrm{L})}{\mathrm{Mg}}$ & $\begin{array}{c}\mathrm{Na} \\
(\mu \mathrm{eq} / \mathrm{L})\end{array}$ & $\begin{array}{c}K \\
\text { (peq/L) }\end{array}$ & 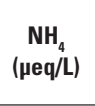 & $\begin{array}{c}\text { Cl } \\
(\mu \mathrm{eq} / \mathrm{L})\end{array}$ & $\begin{array}{c}\mathrm{SO}_{4} \\
(\mu \mathrm{eq} / \mathrm{L})\end{array}$ & $\begin{array}{c}\mathrm{NO}_{3} \\
(\mu \mathrm{eq} / \mathrm{L})\end{array}$ & $\begin{array}{l}\text { DOC } \\
\text { (mg/L) }\end{array}$ & $\begin{array}{l}\text { SWE } \\
\text { (cm) }\end{array}$ & $\begin{array}{l}\text { Snow } \\
\text { depth } \\
\text { (cm) }\end{array}$ & $\begin{array}{c}\delta^{34} \mathbf{S} \\
\text { (per mil) }\end{array}$ & $\begin{array}{c}\mathrm{Hg} \\
\text { (ng/L) }\end{array}$ & $\begin{array}{c}\text { Ionic } \\
\text { balance } \\
(\%)\end{array}$ \\
\hline $\begin{array}{l}\text { Rabbit Ears 1, } \\
\text { Colo. }\end{array}$ & $3 / 23 / 2002$ & E & -12.5 & 6.3 & 5.01 & 9.8 & 8.0 & $<1.7$ & 1.4 & 0.8 & 4.9 & 1.3 & 8.5 & 14.8 & 0.8 & 57 & 170 & 8.0 & 2.4 & 0.6 \\
\hline $\begin{array}{l}\text { Rabbit Ears 2, } \\
\text { Colo. }\end{array}$ & $3 / 23 / 2002$ & E & -13.9 & 6.5 & 5.02 & 9.5 & 7.0 & 2.5 & 1.1 & $<0.4$ & 5.3 & 1.0 & 7.8 & 13.8 & 0.4 & 63 & 184 & na & 1.1 & 5.6 \\
\hline $\begin{array}{l}\text { Red Mountain, } \\
\text { Mont. }\end{array}$ & $3 / 6 / 2002$ & E & -4.6 & 3.8 & 5.27 & 5.4 & 4.5 & $<1.7$ & $<1.0$ & 0.6 & 4.7 & $<1.0$ & 2.4 & 6.1 & 0.7 & 33 & 121 & na & 3.7 & 27.8 \\
\hline $\begin{array}{l}\text { Red Mountain } \\
\text { Pass, Colo. }\end{array}$ & $3 / 27 / 2002$ & E & 36.8 & 7.3 & 6.51 & 0.3 & 37.9 & 3.3 & 3.0 & 1.4 & 3.5 & 2.7 & 5.2 & 13.7 & 0.5 & 36 & 123 & 4.9 & 4.6 & -8.3 \\
\hline $\begin{array}{l}\text { Rendezvous } \\
\text { Mountain, } \\
\text { Wyo. }\end{array}$ & $3 / 18 / 2002$ & E & -7.1 & 3.5 & 5.47 & 3.4 & 4.0 & $<1.7$ & 1.6 & $<0.4$ & 6.6 & $<1.0$ & 4.9 & 5.9 & $<0.4$ & 72 & 240 & na & 0.6 & 18.2 \\
\hline $\begin{array}{l}\text { Slumgullion } \\
\text { Pass, Colo. }\end{array}$ & $3 / 26 / 2002$ & E & 42.0 & 7.9 & 6.52 & 0.3 & 45.4 & 4.9 & 2.6 & 3.4 & 2.5 & 2.3 & 6.6 & 12.8 & 1.2 & 16 & 66 & 4.3 & 6.5 & -3.7 \\
\hline $\begin{array}{l}\text { Snow Bowl, } \\
\text { Mont. }\end{array}$ & $3 / 9 / 2002$ & E & -6.4 & 3.7 & 5.27 & 5.4 & $<3.1$ & $<1.7$ & $<1.0$ & 0.7 & 3.1 & $<1.0$ & 3.3 & 4.4 & 0.5 & 82 & 267 & na & 11.0 & 8.0 \\
\hline South Pass, Wyo. & $3 / 25 / 2002$ & E & -4.7 & 7.4 & 5.33 & 4.7 & 15.0 & 3.3 & 5.0 & 2.7 & 7.1 & 1.9 & 15.5 & 12.5 & 1.1 & 27 & 101 & na & 11.5 & 11.6 \\
\hline $\begin{array}{l}\text { Spring Gulch, } \\
\text { Mont. }\end{array}$ & $3 / 1 / 2002$ & E & -0.2 & 4.3 & 5.62 & 2.4 & 13.5 & $<1.7$ & $<1.0$ & 1.2 & 7.7 & $<1.0$ & 3.2 & 12.0 & 0.6 & 14 & 60 & na & 5.3 & 24.0 \\
\hline $\begin{array}{l}\text { Sunlight Peak, } \\
\text { Colo. }\end{array}$ & $3 / 22 / 2002$ & $\mathrm{E}$ & 38.7 & 8.1 & 6.47 & 0.3 & 49.4 & 4.1 & 1.6 & 1.2 & 5.3 & 1.3 & 6.7 & 14.3 & 0.5 & 41 & 147 & 5.7 & 3.7 & 0.8 \\
\hline $\begin{array}{l}\text { Swiftcurrent } 1 \\
\quad \text { (Glacier NP), } \\
\text { Mont. }\end{array}$ & $3 / 19 / 2002$ & E & -7.7 & 3.9 & 5.18 & 6.6 & $<3.1$ & $<1.7$ & $<1.0$ & 0.5 & 4.9 & $<1.0$ & 4.1 & 7.2 & $<0.4$ & 63 & 201 & na & 3.1 & 3.4 \\
\hline $\begin{array}{l}\text { Sylvan Lake, } \\
\text { Wyo. }\end{array}$ & $2 / 26 / 2002$ & E & -3.6 & 3.5 & 5.44 & 3.6 & $<3.1$ & $<1.7$ & 1.1 & $<0.4$ & 5.4 & $<1.0$ & 3.4 & 6.3 & $<0.4$ & 48 & 160 & na & 1.8 & 2.6 \\
\hline $\begin{array}{l}\text { Sylvan Lake (in } \\
\text { road), Wyo. }\end{array}$ & $2 / 26 / 2002$ & E & -3.4 & 3.8 & 5.57 & 2.7 & 6.0 & $<1.7$ & 4.1 & $<0.4$ & 4.5 & 1.8 & 5.9 & 6.9 & 0.5 & 38 & 95 & na & 1.7 & 8.3 \\
\hline $\begin{array}{l}\text { Taos Ski Valley, } \\
\text { N. Mex. }\end{array}$ & $3 / 16 / 2002$ & $\mathrm{E}$ & 8.7 & 7.9 & 5.95 & 1.1 & 29.4 & 4.1 & 4.1 & 4.4 & 7.2 & 3.2 & 12.8 & 20.1 & 1.8 & 35 & 125 & 5.2 & 11.9 & 5.8 \\
\hline Teton Pass, Wyo. & $3 / 26 / 2002$ & E & -0.6 & 4.6 & 5.70 & 2.0 & 8.5 & 2.5 & 2.5 & 1.7 & 9.3 & 1.4 & 6.8 & 9.2 & 0.8 & 37 & 154 & na & 4.8 & 20.6 \\
\hline $\begin{array}{l}\text { Togwotee Pass, } \\
\text { Wyo. }\end{array}$ & $3 / 27 / 2002$ & E & -2.8 & 3.6 & 5.64 & 2.3 & 6.5 & $<1.7$ & 2.1 & 0.9 & 5.4 & 1.3 & 4.7 & 6.8 & 0.5 & 52 & 203 & na & 3.8 & 14.6 \\
\hline $\begin{array}{l}\text { Twenty-one Mile, } \\
\text { Mont. }\end{array}$ & $3 / 4 / 2002$ & E & -5.5 & 3.9 & 5.33 & 4.7 & $<3.1$ & $<1.7$ & $<1.0$ & 0.4 & 6.8 & 1.3 & 3.0 & 8.0 & $<0.4$ & 30 & 110 & na & 2.6 & -1.4 \\
\hline $\begin{array}{l}\text { University Camp, } \\
\text { Colo. }\end{array}$ & $4 / 2 / 2002$ & E & 0.5 & 5.4 & 5.71 & 1.9 & 15.5 & 2.5 & 1.0 & 1.6 & 11.3 & 1.1 & 7.7 & 16.2 & 0.9 & 13 & 51 & na & 2.1 & 14.0 \\
\hline
\end{tabular}


[E, depth-integrated environmental; ANC, acid neutralization capacity; $\mu \mathrm{eq} / \mathrm{L}$, microequivalents per liter; $\mathrm{uS} / \mathrm{cm}$, microsiemens per centimeter; $\mathrm{SC}$, specific conductance at $25^{\circ} \mathrm{C} ; \mathrm{pH}$ values in standard units; $\mathrm{H}$, hydrogen; Ca, calcium; Mg, magnesium; $\mathrm{Na}$, sodium; $\mathrm{K}$, potassium; $\mathrm{NH}_{4}$, ammonium; $\mathrm{Cl}$, chloride; $\mathrm{SO}_{4}$, sulfate; $\mathrm{NO}_{3}$, nitrate; DOC, dissolved organic carbon; mg/L, milligrams per liter; $\mathrm{SWE}$, snow/ water equivalent; cm, centimeters; $\delta^{34} \mathrm{~S}$, stable sulfur isotope ratio $\left({ }^{34} \mathrm{~S} /{ }^{32} \mathrm{~S}\right) ; \mathrm{Hg}$, mercury; ng/L, nanograms per liter; na, not analyzed; <, below reporting limits; NP, National Park; (), suspect ionic balance; $\%$, percent]

\begin{tabular}{|c|c|c|c|c|c|c|c|c|c|c|c|c|c|c|c|c|c|c|c|c|}
\hline Site name & $\begin{array}{l}\text { Sample } \\
\text { date }\end{array}$ & $\begin{array}{l}\text { Sample } \\
\text { type }\end{array}$ & 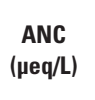 & $\begin{array}{c}\mathrm{SC} \\
(\mu \mathrm{S} / \mathrm{cm})\end{array}$ & $\mathrm{pH}$ & $\begin{array}{c}H \\
(\mu e q / L)\end{array}$ & $\underset{(\mu \mathrm{eq} / \mathrm{L})}{\mathrm{Ca}}$ & $\underset{(\mu \mathrm{eq} / \mathrm{L})}{\mathrm{Mg}}$ & 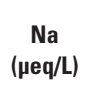 & $\begin{array}{c}K \\
\text { (peq/L) }\end{array}$ & $\begin{array}{c}\mathrm{NH}_{4} \\
(\mu \mathrm{eq} / \mathrm{L})\end{array}$ & $\begin{array}{c}C l \\
(\mu \mathrm{eq} / \mathrm{L})\end{array}$ & $\begin{array}{c}\mathrm{SO}_{4} \\
(\mu \mathrm{eq} / \mathrm{L})\end{array}$ & $\begin{array}{c}\mathrm{NO}_{3} \\
(\mu \mathrm{eq} / \mathrm{L})\end{array}$ & $\begin{array}{l}\text { DOC } \\
(\mathrm{mg} / \mathrm{L})\end{array}$ & $\begin{array}{l}\text { SWE } \\
\text { (cm) }\end{array}$ & $\begin{array}{l}\text { Snow } \\
\text { depth } \\
\text { (cm) }\end{array}$ & $\begin{array}{c}\delta^{34} \mathrm{~S} \\
\text { (per mil) }\end{array}$ & $\underset{\text { (ng/L) }}{\mathrm{Hg}}$ & $\begin{array}{c}\text { lonic } \\
\text { balance } \\
(\%)\end{array}$ \\
\hline $\begin{array}{l}\text { Vallecito Snotel, } \\
\text { Colo. }\end{array}$ & $3 / 22 / 2002$ & E & 14.1 & 5.9 & 6.35 & 0.4 & 27.4 & 4.9 & 1.7 & 5.4 & $<1.0$ & 2.1 & 7.9 & 14.4 & 1.6 & 17 & 73 & 4.7 & 6.2 & 1.8 \\
\hline $\begin{array}{l}\text { W10 (Glacier } \\
\text { NP), Mont. }\end{array}$ & $3 / 12 / 2002$ & E & -7.2 & 3.9 & 5.22 & 6.0 & $<3.1$ & $<1.7$ & $<1.0$ & $<0.4$ & 3.5 & $<1.0$ & 3.4 & 6.5 & 0.4 & 69 & 273 & na & 2.0 & -1.9 \\
\hline $\begin{array}{l}\text { West Yellow- } \\
\text { stone, Mont. }\end{array}$ & $2 / 28 / 2002$ & E & -4.1 & 4.6 & 5.40 & 4.0 & 3.5 & $<1.7$ & 1.7 & 0.6 & 10.3 & $<1.0$ & 5.5 & 10.7 & $<0.4$ & 24 & 91 & na & 2.2 & 10.8 \\
\hline $\begin{array}{l}\text { West Yellowstone } \\
\text { (in road), } \\
\text { Mont. }\end{array}$ & $2 / 28 / 2002$ & E & -1.4 & 10.9 & 5.29 & 5.1 & 15.0 & 3.3 & 8.8 & 2.8 & 31.0 & 6.8 & 18.2 & 13.1 & 4.4 & na & 27 & na & 2.4 & $(26.9)$ \\
\hline $\begin{array}{l}\text { Wolf Creek Pass, } \\
\text { Colo. }\end{array}$ & $3 / 26 / 2002$ & E & 3.7 & 5.6 & 5.91 & 1.2 & 23.0 & 3.3 & 2.6 & 3.3 & 3.7 & 2.4 & 8.2 & 15.4 & 1.2 & 28 & 117 & 4.8 & 6.0 & 11.1 \\
\hline
\end{tabular}


Table 12. Selected chemical and physical data for Rocky Mountain snowpack samples for 2003.

[E, depth-integrated environmental; ANC, acid neutralization capacity; $\mu \mathrm{eq} / \mathrm{L}$, microequivalents per liter; $\mu \mathrm{S} / \mathrm{cm}$, microsiemens per centimeter; $\mathrm{SC}$, specific conductance at $25^{\circ} \mathrm{C} ; \mathrm{pH}$ values in standard units; $\mathrm{H}$, hydrogen; $\mathrm{Ca}$, calcium; $\mathrm{Mg}$, magnesium; $\mathrm{Na}$, sodium; $\mathrm{K}$, potassium; $\mathrm{NH}_{4}$, ammonium; $\mathrm{Cl}$, chloride; $\mathrm{SO}_{4}$, sulfate; $\mathrm{NO}_{3}$, nitrate; DOC, dissolved organic carbon; mg/L, milligrams per liter; $\mathrm{SWE}$, snow/ water equivalent; cm, centimeters; $\delta^{34} \mathrm{~S}$, stable sulfur isotope ratio $\left({ }^{34} \mathrm{~S} /{ }^{22} \mathrm{~S}\right) ; \mathrm{Hg}$, mercury; ng/L, nanograms per liter; na, not analyzed; <, below reporting limits; NP, National Park; (), suspect ionic balance; $\%$, percent]

\begin{tabular}{|c|c|c|c|c|c|c|c|c|c|c|c|c|c|c|c|c|c|c|c|c|}
\hline Site name & $\begin{array}{l}\text { Sample } \\
\text { date }\end{array}$ & $\begin{array}{c}\text { Sample } \\
\text { type }\end{array}$ & $\begin{array}{c}\text { ANC } \\
\text { ( } \mu \mathrm{eq} / \mathrm{L})\end{array}$ & $\begin{array}{c}S C \\
(\mu \mathrm{S} / \mathrm{cm})\end{array}$ & $\mathrm{pH}$ & $\begin{array}{c}H \\
(\mu e q / L)\end{array}$ & $\begin{array}{c}\mathrm{Ca} \\
(\mu \mathrm{eq} / \mathrm{L})\end{array}$ & $\begin{array}{c}\mathrm{Mg} \\
(\mu \mathrm{eq} / \mathrm{L})\end{array}$ & $\underset{(\mu \mathrm{Na} / \mathrm{L})}{\mathrm{Na}}$ & $\begin{array}{c}K \\
(\mu \mathrm{eq} / L)\end{array}$ & $\underset{\text { (peq/L) }}{\mathrm{NH}_{4}}$ & $\begin{array}{c}C I \\
(\mu \mathrm{eq} / \mathrm{L})\end{array}$ & $\begin{array}{c}\mathrm{SO}_{4} \\
(\mu \mathrm{eq} / \mathrm{L})\end{array}$ & $\begin{array}{c}\mathrm{NO}_{3} \\
(\mu \mathrm{eq} / \mathrm{L})\end{array}$ & $\begin{array}{c}\mathrm{DOC} \\
(\mathrm{mg} / \mathrm{L})\end{array}$ & $\begin{array}{l}\text { SWE } \\
(\mathrm{cm})\end{array}$ & $\begin{array}{l}\text { Snow } \\
\text { depth } \\
\text { (cm) }\end{array}$ & $\begin{array}{c}\delta^{34} \mathrm{~S} \\
\text { (per mil) }\end{array}$ & $\begin{array}{c}\mathrm{Hg} \\
(\mathbf{n g} / \mathrm{L})\end{array}$ & $\begin{array}{c}\text { Ionic } \\
\text { balance } \\
(\%)\end{array}$ \\
\hline $\begin{array}{l}\text { Apgar Lookout, } \\
\text { Mont. }\end{array}$ & 3/12/2003 & E & -8.0 & 5.8 & 5.01 & 9.8 & $<3.1$ & $<1.7$ & 1.2 & 0.4 & 6.9 & 1.3 & 4.4 & 6.9 & 0.5 & 50 & 174 & na & 1.3 & 18.6 \\
\hline $\begin{array}{l}\text { Arapaho Creek, } \\
\text { Colo. }\end{array}$ & $3 / 25 / 2003$ & $\mathrm{E}$ & -12.2 & 5.9 & 5.04 & 9.1 & 5.0 & 2.5 & $<1.0$ & 1.2 & 4.8 & $<1.0$ & 4.6 & 12.7 & 0.8 & 25 & 97 & na & 2.4 & 13.4 \\
\hline $\begin{array}{l}\text { Banner Summit, } \\
\text { Idaho }\end{array}$ & 3/10/2003 & E & 6.6 & 3.5 & 5.64 & 2.3 & 7.5 & $<1.7$ & 6.7 & 0.6 & 3.4 & 1.5 & 3.3 & 3.1 & $<0.4$ & 67 & 226 & na & 3.3 & 16.8 \\
\hline Bear Lake, Colo. & 4/8/2003 & E & 1.0 & 4.8 & 5.42 & 3.8 & 11.5 & 2.5 & 1.4 & 1.8 & 8.0 & $<1.0$ & 6.1 & 9.1 & 1.2 & 55 & 194 & na & 4.7 & 28.1 \\
\hline $\begin{array}{l}\text { Berthoud Pass, } \\
\text { Colo. }\end{array}$ & 4/10/2003 & E & -4.6 & 5.3 & 5.28 & 5.2 & 9.0 & 4.9 & 4.6 & 3.1 & 6.1 & 4.2 & 4.2 & 10.7 & 1.6 & 64 & 198 & na & 3.5 & 26.7 \\
\hline $\begin{array}{l}\text { Big Mountain, } \\
\text { Mont. }\end{array}$ & $3 / 7 / 2003$ & $\mathrm{E}$ & -5.8 & 3.3 & 5.24 & 5.8 & $<3.1$ & $<1.7$ & 1.2 & $<0.4$ & 4.0 & $<1.0$ & 2.7 & 4.6 & 0.4 & 68 & 258 & na & 1.3 & 20.3 \\
\hline Big Sky, Mont. & $3 / 18 / 2003$ & $\mathrm{E}$ & 2.7 & 3.2 & 5.74 & 1.8 & 9.5 & 2.5 & 4.8 & 0.8 & 4.6 & 3.0 & 3.8 & 4.9 & 0.5 & 56 & 177 & na & 1.8 & 24.9 \\
\hline $\begin{array}{l}\text { Brooklyn Lake, } \\
\text { Wyo. }\end{array}$ & $3 / 27 / 2003$ & $\mathrm{E}$ & 1.5 & 5.5 & 5.56 & 2.8 & 16.0 & 4.1 & 4.0 & 1.8 & 7.9 & 2.1 & 10.2 & 11.3 & 1.4 & 44 & 157 & 7.3 & 16.3 & 18.5 \\
\hline Brumley, Colo. & $3 / 27 / 2003$ & E & -7.0 & 4.5 & 5.19 & 6.5 & 8.0 & 2.5 & 1.0 & 2.9 & 4.3 & 1.4 & 4.2 & 9.5 & 2.1 & 36 & 115 & na & 2.6 & 24.9 \\
\hline $\begin{array}{l}\text { Buffalo Pass, } \\
\text { Colo. }\end{array}$ & $4 / 11 / 2003$ & E & 1.6 & 5.2 & 5.83 & 1.5 & 19.5 & 4.1 & 2.9 & 1.0 & 7.5 & 1.3 & 11.0 & 13.1 & 0.5 & 127 & 375 & 3.6 & 2.7 & 15.0 \\
\hline $\begin{array}{l}\text { Cameron Pass, } \\
\text { Colo. }\end{array}$ & 3/26/2003 & E & -10.3 & 5.1 & 5.07 & 8.5 & 5.5 & 2.5 & 1.3 & 0.8 & 4.2 & $<1.0$ & 6.0 & 8.9 & 0.5 & 67 & 232 & na & 2.2 & 21.1 \\
\hline Canyon, Wyo. & $2 / 26 / 2003$ & $\mathrm{E}$ & -1.6 & 3.4 & 5.57 & 2.7 & 5.5 & 2.5 & 1.7 & 0.6 & 7.7 & $<1.0$ & 4.6 & 7.1 & $<0.4$ & 27 & 108 & na & 1.3 & 27.5 \\
\hline $\begin{array}{l}\text { Chief Joseph Pass, } \\
\text { Mont. }\end{array}$ & $3 / 9 / 2003$ & $\mathrm{E}$ & -5.4 & 3.3 & 5.24 & 5.8 & $<3.1$ & $<1.7$ & $<1.0$ & $<0.4$ & 2.1 & $<1.0$ & 1.6 & 2.3 & $<0.4$ & 60 & 223 & na & 1.8 & 33.9 \\
\hline Daisy Pass, Mont. & $3 / 4 / 2003$ & E & -3.3 & 3.2 & 5.41 & 3.9 & $<3.1$ & $<1.7$ & $<1.0$ & $<0.4$ & 7.2 & $<1.0$ & 3.6 & 5.4 & $<0.4$ & 72 & 250 & na & 1.4 & 10.7 \\
\hline $\begin{array}{l}\text { Deadman Pass, } \\
\text { Colo. }\end{array}$ & $3 / 28 / 2003$ & E & -6.4 & 4.4 & 5.25 & 5.6 & 7.0 & 2.5 & 1.3 & 2.5 & 5.4 & $<1.0$ & 5.8 & 8.3 & 1.5 & 41 & 166 & na & 1.2 & 26.6 \\
\hline Divide Peak, Wyo. & $3 / 26 / 2003$ & E & -7.8 & 6.7 & 5.16 & 6.9 & 10.5 & 2.5 & 3.6 & 1.6 & 8.5 & 1.2 & 11.3 & 16.2 & 1.1 & 62 & 178 & na & 1.9 & 7.8 \\
\hline Dry Lake, Colo. & $3 / 25 / 2003$ & E & -9.4 & 7.1 & 5.06 & 8.7 & 11.0 & 3.3 & 2.1 & 5.3 & 6.8 & 1.5 & 10.0 & 16.9 & 1.0 & 51 & 162 & 6.6 & 2.1 & 13.5 \\
\hline $\begin{array}{l}\text { Dunckley Pass, } \\
\text { Colo. }\end{array}$ & $4 / 3 / 2003$ & E & 13.1 & 4.8 & 6.13 & 0.7 & 23.0 & 4.9 & 2.0 & 1.9 & 6.9 & $<1.0$ & 6.5 & 10.5 & 0.7 & 62 & 207 & na & 1.9 & 13.3 \\
\hline $\begin{array}{l}\text { E5 (Glacier NP), } \\
\quad \text { Mont. }\end{array}$ & 3/27/2003 & E & -1.9 & 3.0 & 5.40 & 4.0 & 4.0 & $<1.7$ & 2.4 & 1.6 & 3.8 & 1.2 & 3.6 & 3.9 & 0.8 & 112 & 357 & na & 3.1 & 29.0 \\
\hline Elk River, Colo. & $3 / 24 / 2003$ & E & -6.5 & 5.5 & 5.26 & 5.5 & 13.0 & 3.3 & 3.2 & 2.0 & 7.0 & 1.0 & 7.6 & 13.6 & 1.0 & 40 & 135 & na & 3.1 & 21.1 \\
\hline $\begin{array}{c}\text { Elkhart Park, } \\
\text { Wyo. }\end{array}$ & 3/25/2003 & E & 0.9 & 3.9 & 5.59 & 2.6 & 8.5 & 4.1 & 3.2 & 0.9 & 8.7 & 1.3 & 6.9 & 7.7 & 0.6 & 32 & 125 & na & 1.1 & 25.0 \\
\hline
\end{tabular}


[E, depth-integrated environmental; ANC, acid neutralization capacity; $\mu \mathrm{eq} / \mathrm{L}$, microequivalents per liter; $\mu \mathrm{S} / \mathrm{cm}$, microsiemens per centimeter; $\mathrm{SC}$, specific conductance at $25^{\circ} \mathrm{C}$; $\mathrm{pH}$ values in standard units; $\mathrm{H}$, hydrogen; Ca, calcium; Mg, magnesium; $\mathrm{Na}$, sodium; $\mathrm{K}$, potassium; $\mathrm{NH}_{4}$, ammonium; $\mathrm{Cl}$, chloride; $\mathrm{SO}_{4}$, sulfate; $\mathrm{NO}_{3}$, nitrate; DOC, dissolved organic carbon; mg/L, milligrams per liter; $\mathrm{SWE}$, snow/ water equivalent; cm, centimeters; $\delta^{34} \mathrm{~S}$, stable sulfur isotope ratio $\left({ }^{34} \mathrm{~S} /{ }^{22} \mathrm{~S}\right) ; \mathrm{Hg}$, mercury; ng/L, nanograms per liter; na, not analyzed; <, below reporting limits; NP, National Park; (), suspect ionic balance; $\%$, percent]

\begin{tabular}{|c|c|c|c|c|c|c|c|c|c|c|c|c|c|c|c|c|c|c|c|c|}
\hline Site name & $\begin{array}{l}\text { Sample } \\
\text { date }\end{array}$ & $\begin{array}{l}\text { Sample } \\
\text { type }\end{array}$ & $\begin{array}{c}\text { ANC } \\
\text { ( } \mu \mathrm{eq} / \mathrm{L})\end{array}$ & $\begin{array}{c}\mathrm{SC} \\
(\mu \mathrm{S} / \mathrm{cm})\end{array}$ & $\mathrm{pH}$ & 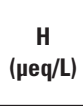 & $\begin{array}{c}\mathrm{Ca} \\
(\mu \mathrm{eq} / \mathrm{L})\end{array}$ & $\begin{array}{c}M g \\
(\mu e q / L)\end{array}$ & 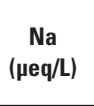 & 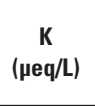 & $\begin{array}{c}\mathrm{NH}_{4} \\
(\mu \mathrm{eq} / \mathrm{L})\end{array}$ & 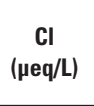 & $\underset{(\mu \mathrm{eq} / \mathrm{L})}{\mathrm{SO}_{4}}$ & $\begin{array}{c}\mathrm{NO}_{3} \\
(\mu \mathrm{eq} / \mathrm{L})\end{array}$ & $\begin{array}{l}\text { DOC } \\
\text { (mg/L) }\end{array}$ & $\begin{array}{l}\text { SWE } \\
(\mathrm{cm})\end{array}$ & $\begin{array}{l}\text { Snow } \\
\text { depth } \\
\text { (cm) }\end{array}$ & $\begin{array}{c}\delta^{34} \mathbf{S} \\
\text { (per mil) }\end{array}$ & $\underset{\text { (ng/L) }}{\mathrm{Hg}}$ & $\begin{array}{c}\text { lonic } \\
\text { balance } \\
(\%)\end{array}$ \\
\hline $\begin{array}{l}\text { Four Mile } \\
\quad \text { Meadow, Wyo. }\end{array}$ & $3 / 26 / 2003$ & E & 5.7 & 3.7 & 5.78 & 1.7 & 10.0 & 2.5 & 2.4 & 1.3 & 7.2 & $<1.0$ & 4.1 & 6.8 & 0.9 & 26 & 107 & na & 2.9 & 20.4 \\
\hline $\begin{array}{l}\text { Fremont Pass, } \\
\text { Colo. }\end{array}$ & $4 / 17 / 2003$ & E & -0.3 & 3.5 & 5.66 & 2.2 & 12.5 & 3.3 & 1.1 & 1.5 & 3.5 & 1.1 & 4.2 & 8.5 & 0.6 & 37 & 147 & na & 3.5 & 26.9 \\
\hline $\begin{array}{l}\text { Galena Summit, } \\
\text { Idaho }\end{array}$ & $3 / 10 / 2003$ & E & -0.8 & 2.6 & 5.69 & 2.0 & 8.5 & 5.8 & 3.0 & 0.8 & 3.6 & 1.2 & 3.2 & 3.7 & 0.6 & 53 & 186 & 6.4 & 2.4 & 49.1 \\
\hline $\begin{array}{l}\text { Garnet Canyon, } \\
\text { Wyo. }\end{array}$ & $3 / 28 / 2003$ & E & 18.0 & 4.9 & 6.14 & 0.7 & 21.0 & 4.9 & 4.0 & 3.1 & 8.6 & 1.1 & 6.8 & 6.2 & 0.7 & 60 & 225 & na & 4.3 & 13.6 \\
\hline $\begin{array}{l}\text { Grand Mesa, } \\
\text { Colo. }\end{array}$ & $3 / 24 / 2003$ & E & 1.5 & 5.4 & 5.61 & 2.5 & 20.0 & 6.6 & 2.9 & 5.5 & 8.1 & 1.7 & 7.5 & 13.3 & 2.1 & 40 & 143 & na & 5.2 & $(31.0)$ \\
\hline $\begin{array}{l}\text { Granite Park, } \\
\text { Mont. }\end{array}$ & $4 / 8 / 2003$ & E & -2.2 & 2.5 & 5.52 & 3.0 & $<3.1$ & $<1.7$ & 1.3 & $<0.4$ & 3.6 & $<1.0$ & 1.7 & 3.4 & $<0.4$ & 129 & 345 & na & 3.5 & 21.7 \\
\hline $\begin{array}{l}\text { Granite Pass, } \\
\text { Mont. }\end{array}$ & $3 / 9 / 2003$ & E & -2.5 & 3.7 & 5.17 & 6.8 & $<3.1$ & $<1.7$ & $<1.0$ & 0.9 & 2.3 & $<1.0$ & 1.6 & 2.4 & 0.5 & 68 & 252 & na & 3.3 & 43.1 \\
\hline $\begin{array}{l}\text { Green Ridge, } \\
\text { Colo. }\end{array}$ & $3 / 25 / 2003$ & E & -1.3 & 3.8 & 5.58 & 2.6 & 10.0 & 3.3 & 1.2 & 1.8 & 4.1 & 1.0 & 4.0 & 10.7 & 0.7 & 24 & 85 & na & 1.6 & 18.9 \\
\hline $\begin{array}{l}\text { Gypsum Creek, } \\
\text { Wyo. }\end{array}$ & $3 / 26 / 2003$ & E & 13.2 & 5.4 & 6.20 & 0.6 & 21.0 & 4.1 & 8.8 & 0.8 & 7.2 & 2.9 & 10.0 & 6.7 & 0.6 & 32 & 116 & na & 2.2 & 12.9 \\
\hline $\begin{array}{l}\text { Hopewell, } \\
\text { N. Mex. }\end{array}$ & $3 / 28 / 2003$ & E & 12.5 & 4.9 & 6.22 & 0.6 & 26.4 & 3.3 & 2.0 & 1.1 & 5.4 & 1.3 & 7.3 & 12.1 & 0.6 & 34 & 124 & na & 2.6 & 7.9 \\
\hline Kings Hill, Mont. & $3 / 1 / 2003$ & E & -0.5 & 3.9 & 5.51 & 3.1 & 7.0 & 2.5 & 2.3 & 1.2 & 7.1 & 1.1 & 5.9 & 8.5 & 0.8 & 30 & 102 & 9.5 & 5.4 & 19.8 \\
\hline Lake, Wyo. & $2 / 26 / 2003$ & E & -3.9 & 3.3 & 5.43 & 3.7 & 4.0 & $<1.7$ & 1.3 & 0.4 & 5.9 & $<1.0$ & 3.7 & 6.4 & 0.5 & 25 & 102 & na & 2.1 & 21.0 \\
\hline $\begin{array}{l}\text { Lake Irene Forest, } \\
\text { Colo. }\end{array}$ & $4 / 1 / 2003$ & E & -5.5 & 5.2 & 5.23 & 5.9 & 9.0 & 4.9 & 1.2 & 5.3 & 4.6 & 2.1 & 6.9 & 10.2 & 1.6 & 65 & 231 & na & 4.1 & 23.2 \\
\hline $\begin{array}{l}\text { Lake Irene } \\
\quad \text { Meadow, Colo. }\end{array}$ & $4 / 1 / 2003$ & E & -10.0 & 5.4 & 5.07 & 8.5 & 4.0 & $<1.7$ & $<1.0$ & $<0.4$ & 4.9 & $<1.0$ & 5.5 & 9.8 & 0.4 & 76 & 226 & na & 1.7 & 6.0 \\
\hline $\begin{array}{l}\text { Lewis Lake } \\
\text { Divide, Wyo. }\end{array}$ & $2 / 28 / 2003$ & E & -2.5 & 3.8 & 5.46 & 3.5 & 3.5 & $<1.7$ & 1.3 & $<0.4$ & 8.5 & $<1.0$ & 3.8 & 6.2 & $<0.4$ & 74 & 227 & na & 1.3 & 25.4 \\
\hline Lionshead, Mont. & $3 / 3 / 2003$ & E & 2.4 & 6.3 & 5.73 & 1.9 & 9.0 & 2.5 & 2.3 & 1.6 & 22.6 & 1.7 & 9.7 & 18.3 & 0.6 & 45 & 146 & na & 4.4 & 10.6 \\
\hline $\begin{array}{l}\text { Loch Vale Forest, } \\
\text { Colo. }\end{array}$ & $4 / 8 / 2003$ & E & 0.3 & 6.5 & 5.21 & 6.2 & 12.5 & 3.3 & 2.5 & 2.0 & 6.9 & 2.4 & 8.5 & 10.4 & 1.0 & 112 & 324 & 7.3 & 2.9 & 21.3 \\
\hline $\begin{array}{l}\text { Loch Vale } \\
\quad \text { Meadow, Colo. }\end{array}$ & $4 / 9 / 2003$ & E & 6.4 & 3.9 & 5.82 & 1.5 & 14.5 & 2.5 & 1.1 & $<0.4$ & 6.1 & $<1.0$ & 5.9 & 9.5 & $<0.4$ & 82 & 235 & 5.9 & 0.9 & 8.0 \\
\hline Lone Pine, Colo. & $4 / 2 / 2003$ & $\mathrm{E}$ & -11.9 & 5.5 & 5.09 & 8.1 & 6.0 & $<1.7$ & $<1.0$ & 0.5 & 4.8 & $<1.0$ & 5.4 & 11.1 & $<0.4$ & 40 & 157 & na & 2.7 & 8.3 \\
\hline
\end{tabular}


Table 12. Selected chemical and physical data for Rocky Mountain snowpack samples for 2003.—Continued

[E, depth-integrated environmental; ANC, acid neutralization capacity; $\mu \mathrm{eq} / \mathrm{L}$, microequivalents per liter; $\mu \mathrm{S} / \mathrm{cm}$, microsiemens per centimeter; $\mathrm{SC}$, specific conductance at $25^{\circ} \mathrm{C} ; \mathrm{pH}$ values in standard units; $\mathrm{H}$, hydrogen; Ca, calcium; Mg, magnesium; $\mathrm{Na}$, sodium; $\mathrm{K}$, potassium; $\mathrm{NH}_{4}$, ammonium; $\mathrm{Cl}$, chloride; $\mathrm{SO}_{4}$, sulfate; $\mathrm{NO}_{3}$, nitrate; DOC, dissolved organic carbon; mg/L, milligrams per liter; $\mathrm{SWE}$, snow/ water equivalent; cm, centimeters; $\delta^{34} \mathrm{~S}$, stable sulfur isotope ratio $\left({ }^{34} \mathrm{~S} /{ }^{22} \mathrm{~S}\right) ; \mathrm{Hg}$, mercury; ng/L, nanograms per liter; na, not analyzed; <, below reporting limits; NP, National Park; (), suspect ionic balance; $\%$, percent]

\begin{tabular}{|c|c|c|c|c|c|c|c|c|c|c|c|c|c|c|c|c|c|c|c|c|}
\hline Site name & $\begin{array}{l}\text { Sample } \\
\text { date }\end{array}$ & $\begin{array}{l}\text { Sample } \\
\text { type }\end{array}$ & $\begin{array}{c}\text { ANC } \\
(\mu e q / L)\end{array}$ & $\begin{array}{c}\text { SC } \\
(\mu \mathrm{S} / \mathrm{cm})\end{array}$ & $\mathrm{pH}$ & 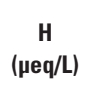 & $\begin{array}{c}\mathrm{Ca} \\
(\mu \mathrm{eq} / \mathrm{L})\end{array}$ & $\underset{(\mu \mathrm{eq} / \mathrm{L})}{\mathrm{Mg}}$ & $\begin{array}{c}\mathrm{Na} \\
(\mu \mathrm{eq} / \mathrm{L})\end{array}$ & $\begin{array}{c}K \\
(\mu e q / L)\end{array}$ & $\begin{array}{c}\mathrm{NH}_{4} \\
\text { ( } \mu \mathrm{eq} / \mathrm{L} \text { ) }\end{array}$ & $\begin{array}{c}C l \\
(\mu \mathrm{eq} / \mathrm{L})\end{array}$ & $\begin{array}{c}\mathrm{SO}_{4} \\
(\mu \mathrm{eq} / \mathrm{L})\end{array}$ & 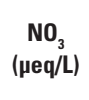 & $\begin{array}{c}\text { DOC } \\
\text { (mg/L) }\end{array}$ & $\begin{array}{l}\text { SWE } \\
\text { (cm) }\end{array}$ & $\begin{array}{l}\text { Snow } \\
\text { depth } \\
\text { (cm) }\end{array}$ & $\begin{array}{c}\delta^{34} \mathbf{S} \\
\text { (per mil) }\end{array}$ & $\begin{array}{c}\mathrm{Hg} \\
\text { (ng/L) }\end{array}$ & $\begin{array}{c}\text { lonic } \\
\text { balance } \\
(\%)\end{array}$ \\
\hline $\begin{array}{l}\text { Loveland Pass, } \\
\text { Colo. }\end{array}$ & $4 / 23 / 2003$ & E & -0.3 & 3.9 & 5.67 & 2.1 & 12.5 & 3.3 & 2.6 & 0.9 & 5.0 & 2.5 & 5.2 & 9.4 & 0.6 & 63 & 201 & na & 3.6 & 21.7 \\
\hline Lynx Pass, Colo. & $3 / 25 / 2003$ & E & -11.4 & 5.9 & 4.99 & 10.2 & 5.5 & $<1.7$ & $<1.0$ & 0.5 & 4.6 & $<1.0$ & 4.9 & 12.5 & $<0.4$ & 38 & 129 & na & 0.8 & 8.8 \\
\hline Mills Lake, Colo. & 4/8/2003 & E & 3.9 & 6.0 & 5.55 & 2.8 & 20.0 & 4.1 & 2.7 & 1.9 & 8.5 & 1.5 & 10.3 & 14.8 & 1.1 & 91 & 265 & na & 4.8 & 13.5 \\
\hline Molas Lake, Colo. & $3 / 29 / 2003$ & $\mathrm{E}$ & 9.6 & 6.1 & 5.77 & 1.7 & 20.5 & 4.1 & 10.1 & 6.5 & 4.0 & 10.3 & 5.7 & 10.6 & 1.2 & 29 & 88 & 6.0 & 3.1 & 12.9 \\
\hline $\begin{array}{l}\text { Monarch Pass, } \\
\text { Colo. }\end{array}$ & $3 / 27 / 2003$ & $\mathrm{E}$ & -2.4 & 5.1 & 5.30 & 5.0 & 12.0 & 4.1 & 1.2 & 1.7 & 5.1 & 1.4 & 5.0 & 10.5 & 0.7 & 46 & 134 & 5.7 & 3.0 & 26.5 \\
\hline $\begin{array}{l}\text { Monida Pass, } \\
\text { Mont. }\end{array}$ & $2 / 27 / 2003$ & E & 18.5 & 4.5 & 6.31 & 0.5 & 16.0 & 3.3 & 3.2 & 0.8 & 9.1 & 2.3 & 3.6 & 7.6 & 0.4 & 25 & 90 & na & 0.9 & 1.6 \\
\hline $\begin{array}{l}\text { Mount Belmont, } \\
\text { Mont. }\end{array}$ & $3 / 3 / 2003$ & E & -1.2 & 4.4 & 5.37 & 4.3 & 7.5 & 2.5 & 1.4 & 2.3 & 8.9 & $<1.0$ & 5.7 & 7.4 & 1.8 & 29 & 91 & na & 4.7 & 34.4 \\
\hline Ned Wilson, Colo. & $5 / 13 / 2003$ & E & 9.6 & 4.5 & 6.20 & 0.6 & 21.5 & 3.3 & 1.6 & 0.4 & 5.4 & $<1.0$ & 6.1 & 9.3 & 0.4 & 98 & 270 & na & 4.8 & 13.6 \\
\hline $\begin{array}{l}\text { Niwot Snotel, } \\
\text { Colo. }\end{array}$ & $3 / 28 / 2003$ & E & -6.4 & 5.3 & 5.22 & 6.0 & 7.0 & $<1.7$ & 1.1 & 0.8 & 10.5 & $<1.0$ & 8.1 & 12.0 & 0.8 & 41 & 180 & na & 1.5 & 11.8 \\
\hline $\begin{array}{l}\text { Noisy Basin, } \\
\text { Mont. }\end{array}$ & 3/6/2003 & E & -4.4 & 3.6 & 5.22 & 6.0 & $<3.1$ & $<1.7$ & 1.0 & 0.6 & 5.3 & $<1.0$ & 3.4 & 6.0 & 0.4 & 53 & 238 & na & 1.3 & 15.6 \\
\hline Old Battle, Wyo. & $3 / 26 / 2003$ & $\mathrm{E}$ & -1.9 & 5.9 & 5.29 & 5.1 & 10.0 & 2.5 & 2.5 & 2.3 & 7.9 & 1.1 & 8.7 & 11.6 & 0.7 & 81 & 253 & na & 2.1 & 17.4 \\
\hline $\begin{array}{l}\text { Old Faithful Fire } \\
\text { Road, Wyo. }\end{array}$ & $2 / 27 / 2003$ & E & 8.7 & 4.7 & 5.91 & 1.2 & 13.5 & 3.3 & 7.2 & 0.7 & 10.7 & 3.2 & 7.9 & 8.9 & 0.4 & 26 & 100 & na & 1.8 & 12.2 \\
\hline $\begin{array}{l}\text { Old Faithful (in } \\
\text { road), Wyo. }\end{array}$ & $2 / 27 / 2003$ & E & 13.2 & 5.5 & 5.56 & 2.8 & 14.5 & 4.1 & 4.3 & 2.6 & 8.9 & 5.4 & 4.0 & 4.5 & 2.4 & na & 22 & na & 10.5 & 15.6 \\
\hline $\begin{array}{l}\text { Oldman Lake, } \\
\text { Mont. }\end{array}$ & $3 / 17 / 2003$ & E & -3.5 & 4.7 & 5.14 & 7.2 & 3.5 & $<1.7$ & 1.3 & 0.8 & 3.6 & 1.2 & 2.8 & 3.8 & 0.4 & 73 & 203 & na & 1.8 & 35.4 \\
\hline $\begin{array}{l}\text { Phantom Valley, } \\
\text { Colo. }\end{array}$ & $3 / 27 / 2003$ & E & -9.1 & 5.7 & 5.10 & 7.9 & 8.5 & $<1.7$ & 1.2 & 1.2 & 6.6 & $<1.0$ & 7.5 & 12.8 & 0.8 & 29 & 117 & na & 2.0 & 11.1 \\
\hline $\begin{array}{l}\text { Rabbit Ears 1, } \\
\text { Colo. }\end{array}$ & $3 / 25 / 2003$ & E & -8.2 & 6.5 & 5.10 & 7.9 & 8.0 & 3.3 & 1.9 & 3.1 & 8.2 & 1.1 & 8.6 & 12.3 & 1.0 & 67 & 192 & 7.4 & 2.8 & 19.3 \\
\hline $\begin{array}{l}\text { Rabbit Ears 2, } \\
\text { Colo. }\end{array}$ & $3 / 25 / 2003$ & E & -12.9 & 7.9 & 4.97 & 10.7 & 7.5 & 2.5 & 1.9 & 1.4 & 6.4 & $<1.0$ & 8.1 & 12.0 & 1.1 & 65 & 194 & 7.3 & 1.7 & 20.3 \\
\hline $\begin{array}{l}\text { Red Mountain, } \\
\text { Mont. }\end{array}$ & $3 / 5 / 2003$ & E & -0.5 & 3.2 & 5.54 & 2.9 & 6.5 & $<1.7$ & 2.5 & 0.8 & 5.2 & $<1.0$ & 3.8 & 5.3 & 0.6 & 33 & 125 & na & 4.3 & 32.5 \\
\hline $\begin{array}{l}\text { Red Mountain } \\
\text { Pass, Colo. }\end{array}$ & $3 / 29 / 2003$ & E & 0.7 & 2.8 & 5.77 & 1.7 & 11.5 & 2.5 & 1.1 & 0.7 & 2.5 & 1.0 & 3.2 & 6.0 & 0.4 & 60 & 180 & 4.7 & 0.7 & 29.0 \\
\hline
\end{tabular}


[E, depth-integrated environmental; ANC, acid neutralization capacity; $\mu \mathrm{eq} / \mathrm{L}$, microequivalents per liter; $\mu \mathrm{S} / \mathrm{cm}$, microsiemens per centimeter; $\mathrm{SC}$, specific conductance at $25^{\circ} \mathrm{C}$; $\mathrm{pH}$ values in standard units; $\mathrm{H}$, hydrogen; Ca, calcium; Mg, magnesium; $\mathrm{Na}$, sodium; $\mathrm{K}$, potassium; $\mathrm{NH}_{4}$, ammonium; $\mathrm{Cl}$, chloride; $\mathrm{SO}_{4}$, sulfate; $\mathrm{NO}_{3}$, nitrate; DOC, dissolved organic carbon; mg/L, milligrams per liter; $\mathrm{SWE}$, snow/ water equivalent; cm, centimeters; $\delta^{34} \mathrm{~S}$, stable sulfur isotope ratio $\left({ }^{34} \mathrm{~S} /{ }^{22} \mathrm{~S}\right) ; \mathrm{Hg}$, mercury; ng/L, nanograms per liter; na, not analyzed; <, below reporting limits; NP, National Park; (), suspect ionic balance; $\%$, percent]

\begin{tabular}{|c|c|c|c|c|c|c|c|c|c|c|c|c|c|c|c|c|c|c|c|c|}
\hline Site name & $\begin{array}{l}\text { Sample } \\
\text { date }\end{array}$ & $\begin{array}{c}\text { Sample } \\
\text { type }\end{array}$ & $\begin{array}{c}\text { ANC } \\
(\mu \mathrm{eq} / \mathrm{L})\end{array}$ & $\begin{array}{c}\text { SC } \\
(\mu \mathrm{S} / \mathrm{cm})\end{array}$ & $\mathrm{pH}$ & $\begin{array}{c}H \\
\text { (peq/L) }\end{array}$ & $\begin{array}{c}\mathrm{Ca} \\
(\mu \mathrm{eq} / \mathrm{L})\end{array}$ & $\underset{(\mu \mathrm{eq} / \mathrm{L})}{\mathrm{Mg}}$ & $\begin{array}{c}\mathrm{Na} \\
(\mu \mathrm{eq} / \mathrm{L})\end{array}$ & $\begin{array}{c}K \\
(\mu e q / L)\end{array}$ & $\begin{array}{c}\mathrm{NH}_{4} \\
(\mu \mathrm{eq} / \mathrm{L})\end{array}$ & $\underset{(\mu \mathrm{eq} / \mathrm{L})}{\mathrm{Cl}}$ & $\begin{array}{c}\mathrm{SO}_{4} \\
(\mu \mathrm{eq} / \mathrm{L})\end{array}$ & $\begin{array}{c}\mathrm{NO}_{3} \\
(\mu \mathrm{eq} / \mathrm{L})\end{array}$ & $\begin{array}{c}\text { DOC } \\
\text { (mg/L) }\end{array}$ & $\begin{array}{l}\text { SWE } \\
\text { (cm) }\end{array}$ & $\begin{array}{l}\text { Snow } \\
\text { depth } \\
(\mathrm{cm})\end{array}$ & $\begin{array}{c}\delta^{34} \mathbf{S} \\
\text { (per mil) }\end{array}$ & $\underset{\text { (ng/L) }}{\mathrm{Hg}}$ & $\begin{array}{c}\text { lonic } \\
\text { balance } \\
(\%)\end{array}$ \\
\hline $\begin{array}{l}\text { Rendezvous } \\
\text { Mountain, } \\
\text { Wyo. }\end{array}$ & $3 / 27 / 2003$ & $\mathrm{E}$ & 7.0 & 4.0 & 5.64 & 2.3 & 11.0 & 4.9 & 3.3 & 0.5 & 7.3 & 1.4 & 5.3 & 5.1 & $<0.4$ & 90 & 280 & na & 1.6 & 21.8 \\
\hline $\begin{array}{l}\text { Ripple Creek } \\
\text { NADP, Colo. }\end{array}$ & 4/4/2003 & $\mathrm{E}$ & 17.6 & 5.1 & 6.39 & 0.4 & 26.4 & 4.1 & 1.9 & 0.5 & 4.6 & 1.0 & 5.7 & 11.0 & 0.4 & 66 & 208 & na & 1.7 & 3.6 \\
\hline $\begin{array}{l}\text { Slumgullion Pass, } \\
\text { Colo. }\end{array}$ & $3 / 28 / 2003$ & $\mathrm{E}$ & 15.0 & 5.1 & 5.80 & 1.6 & 16.5 & 5.8 & 3.7 & 13.4 & $<1.0$ & 5.7 & 4.7 & 6.8 & 4.0 & 21 & 74 & 5.1 & 11.9 & 11.8 \\
\hline $\begin{array}{c}\text { Snow Bowl, } \\
\text { Mont. }\end{array}$ & $3 / 8 / 2003$ & E & -5.8 & 3.2 & 5.15 & 7.1 & $<3.1$ & $<1.7$ & $<1.0$ & 0.6 & 2.7 & $<1.0$ & 2.4 & 3.3 & 0.4 & 57 & 221 & na & 2.4 & 28.8 \\
\hline $\begin{array}{l}\text { Snyder Lake, } \\
\text { Mont. }\end{array}$ & $3 / 15 / 2003$ & E & -5.9 & 4.1 & 5.21 & 6.2 & $<3.1$ & $<1.7$ & 1.6 & $<0.4$ & 4.8 & $<1.0$ & 2.5 & 4.9 & $<0.4$ & 36 & 170 & na & 2.8 & 26.4 \\
\hline South Pass, Wyo. & 4/1/2003 & E & 1.5 & 3.1 & 5.76 & 1.7 & 8.5 & 2.5 & 2.5 & 1.0 & 6.2 & $<1.0$ & 5.3 & 4.6 & 0.7 & 46 & 144 & na & 2.9 & 32.6 \\
\hline $\begin{array}{l}\text { Spring Gulch, } \\
\text { Mont. }\end{array}$ & $3 / 3 / 2003$ & $\mathrm{E}$ & -1.0 & 3.8 & 5.47 & 3.4 & 8.0 & 4.1 & $<1.0$ & 10.1 & 3.9 & 1.4 & 4.0 & 7.1 & 3.1 & 19 & 67 & 7.4 & 3.3 & 40.3 \\
\hline $\begin{array}{l}\text { Sunlight Peak, } \\
\text { Colo. }\end{array}$ & $3 / 25 / 2003$ & E & -6.3 & 5.3 & 5.21 & 6.2 & 10.0 & 4.9 & 1.3 & 2.0 & 6.3 & 1.2 & 6.4 & 12.6 & 1.6 & 48 & 161 & 5.7 & 2.9 & 20.7 \\
\hline $\begin{array}{l}\text { Sylvan Lake, } \\
\text { Wyo. }\end{array}$ & $2 / 25 / 2003$ & E & -3.8 & 3.4 & 5.41 & 3.9 & $<3.1$ & $<1.7$ & $<1.0$ & 0.6 & 5.8 & $<1.0$ & 3.2 & 5.3 & 0.4 & 50 & 165 & na & 1.3 & 9.8 \\
\hline $\begin{array}{l}\text { Sylvan Lake (in } \\
\text { road), Wyo. }\end{array}$ & $2 / 25 / 2003$ & $\mathrm{E}$ & -5.3 & 4.3 & 5.27 & 5.4 & $<3.1$ & $<1.7$ & $<1.0$ & $<0.4$ & 8.1 & $<1.0$ & 4.6 & 6.7 & 0.4 & 43 & 100 & na & 1.3 & 8.8 \\
\hline $\begin{array}{l}\text { Taos Ski Valley, } \\
\text { N. Mex. }\end{array}$ & $3 / 29 / 2003$ & $\mathrm{E}$ & -3.4 & 3.9 & 5.44 & 3.6 & 9.5 & 2.5 & 1.5 & 0.6 & 5.5 & 1.0 & 6.3 & 9.0 & 0.6 & 79 & 231 & 4.2 & 2.0 & 17.4 \\
\hline Teton Pass, Wyo. & $3 / 26 / 2003$ & E & 24.0 & 6.1 & 6.20 & 0.6 & 24.0 & 5.8 & 5.0 & 3.0 & 9.2 & 2.5 & 7.4 & 8.5 & 1.6 & 54 & 203 & na & 7.8 & 5.8 \\
\hline $\begin{array}{l}\text { Togwotee Pass, } \\
\text { Wyo. }\end{array}$ & $3 / 26 / 2003$ & E & 2.8 & 3.1 & 5.74 & 1.8 & 9.0 & 2.5 & 2.2 & 1.0 & 5.7 & 1.0 & 4.2 & 5.8 & 0.6 & 53 & 218 & na & 2.5 & 23.2 \\
\hline Tower, Colo. & $4 / 11 / 2003$ & E & -2.1 & 4.6 & 5.57 & 2.7 & 14.0 & 3.3 & 1.6 & 0.8 & 7.4 & 1.0 & 9.5 & 11.2 & 0.7 & 102 & 297 & na & 2.5 & 15.7 \\
\hline $\begin{array}{l}\text { Twenty-one Mile, } \\
\text { Mont. }\end{array}$ & $3 / 3 / 2003$ & $\mathrm{E}$ & 0.1 & 4.1 & 5.59 & 2.6 & 7.0 & $<1.7$ & 2.4 & 0.8 & 8.9 & 1.6 & 5.3 & 9.0 & 0.5 & 24 & 94 & na & 2.4 & 14.9 \\
\hline $\begin{array}{l}\text { University Camp, } \\
\text { Colo. }\end{array}$ & 4/7/2003 & E & -1.4 & 5.5 & 5.22 & 6.0 & 10.5 & 2.5 & 1.0 & 1.1 & 7.6 & $<1.0$ & 6.7 & 10.0 & 0.7 & 52 & 179 & na & 2.1 & 26.7 \\
\hline $\begin{array}{l}\text { West Yellowstone, } \\
\text { Mont. }\end{array}$ & 2/28/2003 & E & 0.4 & 4.9 & 5.61 & 2.5 & 9.0 & 2.5 & 2.9 & 1.5 & 14.0 & 2.0 & 7.4 & 12.3 & 0.6 & 21 & 81 & na & 2.1 & 18.6 \\
\hline $\begin{array}{l}\text { West Yellowstone } \\
\text { (in road), } \\
\text { Mont. }\end{array}$ & $2 / 27 / 2003$ & $\mathrm{E}$ & 6.2 & 6.8 & 5.92 & 1.2 & 15.5 & 4.9 & 5.1 & 1.2 & 21.4 & 4.8 & 12.3 & 12.4 & 1.4 & na & 38 & na & 4.3 & 16.0 \\
\hline $\begin{array}{l}\text { Wolf Creek Pass, } \\
\text { Colo. }\end{array}$ & $3 / 28 / 2003$ & E & 2.2 & 4.8 & 5.70 & 2.0 & 18.5 & 3.3 & 4.0 & 3.8 & 4.4 & 1.9 & 9.9 & 10.9 & 1.1 & 68 & 197 & na & 4.0 & 18.2 \\
\hline
\end{tabular}


Table 13. Selected chemical and physical data for Rocky Mountain snowpack samples for 2004.

[E, depth-integrated environmental; ANC, acid neutralization capacity; $\mu$ eq/L, microequivalents per liter; $\mu \mathrm{S} / \mathrm{cm}$, microsiemens per centimeter; $\mathrm{SC}$, specific conductance at $25^{\circ} \mathrm{C} ; \mathrm{pH}$ values in standard units; $\mathrm{H}$, hydrogen; $\mathrm{Ca}$, calcium; $\mathrm{Mg}$, magnesium; $\mathrm{Na}$, sodium; $\mathrm{K}$, potassium; $\mathrm{NH}_{4}$, ammonium; $\mathrm{Cl}$, chloride; $\mathrm{SO}_{4}$, sulfate; $\mathrm{NO}_{3}$, nitrate; DOC, dissolved organic carbon; mg/L, milligrams per liter; $\mathrm{SWE}$, snow/water equivalent; cm, centimeters; NADP, National Atmospheric Deposition Program; $\delta^{34} \mathrm{~S}$, stable sulfur isotope ratio $\left.{ }^{34} \mathrm{~S} /{ }^{2} \mathrm{~S}\right) ; \mathrm{Hg}$, mercury; ng/L, nanograms per liter; na, not analyzed; <, below reporting limits; (), suspect ionic balance\%, percent]

\begin{tabular}{|c|c|c|c|c|c|c|c|c|c|c|c|c|c|c|c|c|c|c|c|c|}
\hline Site name & $\begin{array}{l}\text { Sample } \\
\text { date }\end{array}$ & $\begin{array}{c}\text { Sample } \\
\text { type }\end{array}$ & $\begin{array}{c}\text { ANC } \\
(\mu \mathrm{eq} / \mathrm{L})\end{array}$ & $\begin{array}{c}\text { SC } \\
(\mu \mathrm{S} / \mathrm{cm})\end{array}$ & $\mathrm{pH}$ & $\begin{array}{c}H \\
(\mu \mathrm{eq} / L)\end{array}$ & $\begin{array}{c}\text { Ca } \\
(\mu \mathrm{eq} / \mathrm{L})\end{array}$ & $\begin{array}{c}\mathrm{Mg} \\
(\mu \mathrm{eq} / \mathrm{L})\end{array}$ & $\underset{(\mu \mathrm{eq} / \mathrm{L})}{\mathrm{Na}}$ & $\begin{array}{c}K \\
(\mu e q / L)\end{array}$ & $\begin{array}{c}\mathrm{NH}_{4} \\
(\mu \mathrm{eq} / \mathrm{L})\end{array}$ & $\begin{array}{c}\text { Cl } \\
(\mu \mathrm{eq} / \mathrm{L})\end{array}$ & $\begin{array}{c}\mathrm{SO}_{4} \\
(\mu \mathrm{eq} / \mathrm{L})\end{array}$ & $\begin{array}{c}\mathrm{NO}_{3} \\
(\mu \mathrm{eq} / \mathrm{L})\end{array}$ & $\begin{array}{c}\text { DOC } \\
\text { (mg/L) }\end{array}$ & $\begin{array}{l}\text { SWE } \\
\text { (cm) }\end{array}$ & $\begin{array}{l}\text { Snow } \\
\text { depth } \\
\text { (cm) }\end{array}$ & $\begin{array}{c}\delta^{34} \mathbf{S} \\
\text { (per mil) }\end{array}$ & $\begin{array}{c}\mathrm{Hg} \\
\text { (ng/L) }\end{array}$ & $\begin{array}{c}\text { lonic } \\
\text { balance } \\
(\%)\end{array}$ \\
\hline $\begin{array}{l}\text { Apgar Lookout, } \\
\text { Mont. }\end{array}$ & 3/9/2004 & E & -10.2 & 5.3 & 5.09 & 8.1 & $<3.1$ & $<1.7$ & 1.5 & $<0.4$ & 5.0 & 2.0 & 4.3 & 7.7 & 0.5 & 61 & 177 & na & 1.7 & 2.2 \\
\hline $\begin{array}{l}\text { Banner Sum- } \\
\text { mit, Idaho }\end{array}$ & $3 / 15 / 2004$ & $\mathrm{E}$ & 12.5 & 7.6 & 6.12 & 0.8 & 15.4 & 2.3 & 32.7 & 0.5 & $<1.0$ & 12.5 & 15.7 & 3.0 & $<0.4$ & 64 & 173 & 7.7 & 4.1 & 8.4 \\
\hline $\begin{array}{l}\text { Berthoud Pass, } \\
\text { Colo. }\end{array}$ & 4/7/2004 & E & -13.2 & 5.1 & 5.27 & 5.4 & 6.3 & $<1.7$ & 1.0 & 0.9 & 5.7 & 1.5 & 4.5 & 9.7 & 0.6 & 50 & 166 & na & 4.0 & 10.3 \\
\hline $\begin{array}{l}\text { Big Mountain, } \\
\text { Mont. }\end{array}$ & $3 / 5 / 2004$ & E & -13.5 & 4.6 & 5.12 & 7.6 & $<3.1$ & $<1.7$ & $<1.0$ & 1.2 & 3.3 & 1.5 & 3.6 & 5.3 & 0.6 & 74 & 252 & 5.6 & 1.7 & 8.0 \\
\hline Big Sky, Mont. & $3 / 17 / 2004$ & E & -4.6 & 3.3 & 5.43 & 3.7 & $<3.1$ & $<1.7$ & $<1.0$ & $<0.4$ & 3.0 & $<1.0$ & 3.0 & 4.5 & $<0.4$ & 23 & 77 & na & 0.9 & -4.8 \\
\hline $\begin{array}{l}\text { Brooklyn Lake, } \\
\text { Wyo. }\end{array}$ & $3 / 26 / 2004$ & E & -1.4 & 6.4 & 5.23 & 5.9 & 13.1 & 3.5 & 1.0 & 5.1 & 6.4 & 1.7 & 9.8 & 12.4 & 1.4 & 54 & 147 & 6.3 & 14.0 & 18.8 \\
\hline Brumley, Colo. & $3 / 25 / 2004$ & $\mathrm{E}$ & -5.1 & 5.7 & 5.06 & 8.7 & 8.4 & 2.2 & $<1.0$ & 6.7 & 4.5 & 3.0 & 5.1 & 11.4 & 2.0 & 29 & 79 & na & 7.2 & 22.1 \\
\hline $\begin{array}{l}\text { Buffalo Pass, } \\
\text { Colo. }\end{array}$ & $3 / 31 / 2004$ & $\mathrm{E}$ & -15.4 & 7.5 & 4.99 & 10.2 & 6.4 & $<1.7$ & $<1.0$ & 1.4 & 6.5 & 1.7 & 9.4 & 11.9 & 0.8 & 102 & 279 & na & 2.1 & 3.4 \\
\hline $\begin{array}{l}\text { Cameron Pass, } \\
\text { Colo. }\end{array}$ & $3 / 15 / 2004$ & E & -11.3 & 6.5 & 4.97 & 10.7 & 5.8 & $<1.7$ & 2.1 & 2.0 & 4.9 & 2.8 & 6.7 & 11.8 & 1.0 & 35 & 132 & na & 2.6 & 9.0 \\
\hline Canyon, Wyo. & $2 / 25 / 2004$ & E & -16.6 & 3.2 & 5.12 & 7.6 & $<3.1$ & $<1.7$ & $<1.0$ & $<0.4$ & 3.3 & $<1.0$ & 2.6 & 6.1 & $<0.4$ & 29 & 111 & na & 0.5 & 11.1 \\
\hline $\begin{array}{l}\text { Chief Joseph } \\
\text { Pass, Mont. }\end{array}$ & $3 / 8 / 2004$ & E & -8.0 & 4.0 & 5.20 & 6.3 & $<3.1$ & $<1.7$ & 1.6 & 1.4 & 2.4 & 1.3 & 2.5 & 3.0 & 1.2 & 48 & 176 & 2.9 & 4.2 & 26.5 \\
\hline $\begin{array}{l}\text { Daisy Pass, } \\
\text { Mont. }\end{array}$ & $3 / 2 / 2004$ & E & -5.4 & 4.5 & 5.38 & 4.2 & 3.5 & $<1.7$ & $<1.0$ & 1.2 & 5.1 & $<1.0$ & 3.8 & 4.9 & 0.9 & 73 & 220 & na & 1.1 & 23.1 \\
\hline $\begin{array}{c}\text { Dry Lake, } \\
\text { Colo. }\end{array}$ & $3 / 25 / 2004$ & E & -12.0 & 8.2 & 4.97 & 10.7 & 6.7 & $<1.7$ & $<1.0$ & $<0.4$ & 3.4 & $<1.0$ & 6.9 & 17.0 & 0.4 & 47 & 127 & na & 1.1 & -7.0 \\
\hline $\begin{array}{l}\text { Dunckley Pass, } \\
\text { Colo. }\end{array}$ & 4/1/2004 & E & -3.1 & 4.9 & 5.69 & 2.0 & 14.4 & 2.5 & $<1.0$ & 2.2 & 5.0 & 1.0 & 5.7 & 11.5 & 0.6 & 59 & 174 & na & 1.2 & 18.1 \\
\hline $\begin{array}{l}\text { Elk River, } \\
\text { Colo. }\end{array}$ & $3 / 25 / 2004$ & E & -8.9 & 6.0 & 5.18 & 6.6 & 11.0 & 2.0 & $<1.0$ & 1.0 & 4.4 & $<1.0$ & 7.5 & 14.4 & 0.4 & 43 & 118 & na & 1.7 & 6.4 \\
\hline $\begin{array}{l}\text { Elkhart Park, } \\
\text { Wyo. }\end{array}$ & $3 / 23 / 2004$ & E & -13.1 & 5.8 & 5.06 & 8.7 & 3.4 & $<1.7$ & $<1.0$ & $<0.4$ & 3.3 & $<1.0$ & 5.5 & 7.4 & 0.6 & 36 & 110 & 5.9 & $<0.4$ & 8.8 \\
\hline $\begin{array}{l}\text { Four Mile } \\
\quad \text { Meadow, } \\
\text { Wyo. }\end{array}$ & $3 / 18 / 2004$ & E & -4.8 & 5.2 & 5.28 & 5.2 & 3.4 & $<1.7$ & $<1.0$ & $<0.4$ & 3.4 & $<1.0$ & 2.3 & 6.3 & 0.5 & 23 & 81 & na & 0.8 & 16.8 \\
\hline $\begin{array}{l}\text { Fremont Pass, } \\
\text { Colo. }\end{array}$ & $4 / 8 / 2004$ & E & -2.7 & 4.6 & 5.32 & 4.8 & 6.1 & $<1.7$ & $<1.0$ & 2.9 & 4.8 & $<1.0$ & 4.2 & 9.0 & 0.8 & 29 & 120 & 4.4 & 2.5 & 16.7 \\
\hline
\end{tabular}


[E, depth-integrated environmental; ANC, acid neutralization capacity; $\mu \mathrm{eq} / \mathrm{L}$, microequivalents per liter; $\mu \mathrm{S} / \mathrm{cm}$, microsiemens per centimeter; $\mathrm{SC}$, specific conductance at $25^{\circ} \mathrm{C}$; $\mathrm{pH}$ values in standard units; $\mathrm{H}$, hydrogen; $\mathrm{Ca}$, calcium; $\mathrm{Mg}$, magnesium; $\mathrm{Na}$, sodium; $\mathrm{K}$, potassium; $\mathrm{NH}_{4}$, ammonium; $\mathrm{Cl}$, chloride; $\mathrm{SO}_{4}$, sulfate; $\mathrm{NO}_{3}$, nitrate; DOC, dissolved organic carbon; mg/L, milligrams per liter; $\mathrm{SWE}$, snow/water equivalent; cm, centimeters; NADP, National Atmospheric Deposition Program; $\delta^{34} \mathrm{~S}$, stable sulfur isotope ratio $\left({ }^{34} \mathrm{~S} /{ }^{22} \mathrm{~S}\right) ; \mathrm{Hg}$, mercury; ng/L, nanograms per liter; na, not analyzed; <, below reporting limits; (), suspect ionic balance\%, percent]

\begin{tabular}{|c|c|c|c|c|c|c|c|c|c|c|c|c|c|c|c|c|c|c|c|c|}
\hline Site name & $\begin{array}{c}\text { Sample } \\
\text { date }\end{array}$ & $\begin{array}{c}\text { Sample } \\
\text { type }\end{array}$ & $\begin{array}{c}\text { ANC } \\
\text { ( } \mu \mathrm{eq} / \mathrm{L} \text { ) }\end{array}$ & $\begin{array}{c}\mathrm{SC} \\
(\mu \mathrm{S} / \mathrm{cm})\end{array}$ & $\mathrm{pH}$ & $\underset{(\mu \mathrm{eq} / \mathrm{L})}{\mathbf{H}}$ & $\begin{array}{c}\mathrm{Ca} \\
(\mu \mathrm{eq} / \mathrm{L})\end{array}$ & $\underset{(\mu \mathrm{gg} / \mathrm{L})}{\mathbf{M g}}$ & $\underset{\text { (peq/L) }}{\mathrm{Na}}$ & $\begin{array}{c}\mathbf{K} \\
(\mu \mathrm{eq} / \mathrm{L})\end{array}$ & $\underset{\text { (peq/L) }}{\mathbf{N H}_{4}}$ & $\begin{array}{c}C l \\
(\mu \mathrm{eq} / L)\end{array}$ & $\underset{\text { (peq/L) }}{\mathrm{SO}_{4}}$ & $\begin{array}{c}\mathrm{NO}_{3} \\
(\mu \mathrm{eq} / \mathrm{L})\end{array}$ & $\begin{array}{c}\text { DOC } \\
\text { (mg/L) }\end{array}$ & $\begin{array}{l}\text { SWE } \\
\text { (cm) }\end{array}$ & $\begin{array}{l}\text { Snow } \\
\text { depth } \\
(\mathrm{cm})\end{array}$ & $\begin{array}{c}\delta^{34} \mathrm{~S} \\
\text { (per mil) }\end{array}$ & $\underset{(\mathrm{ng} / \mathrm{L})}{\mathrm{Hg}}$ & $\begin{array}{c}\text { lonic } \\
\text { balance } \\
(\%)\end{array}$ \\
\hline $\begin{array}{l}\text { Galena Sum- } \\
\text { mit, Idaho }\end{array}$ & $3 / 15 / 2004$ & E & 1.9 & 4.3 & 5.52 & 3.0 & 5.0 & $<1.7$ & 10.7 & 0.6 & 2.8 & 4.0 & 6.8 & 3.0 & na & 44 & 145 & 7.6 & 2.7 & 16.8 \\
\hline $\begin{array}{l}\text { Garnet Canyon, } \\
\text { Wyo. }\end{array}$ & $3 / 25 / 2004$ & E & 2.2 & 4.7 & 5.64 & 2.3 & 9.7 & 2.5 & 1.2 & 2.8 & 4.9 & $<1.0$ & 5.3 & 7.5 & 1.4 & 48 & 119 & na & 2.7 & 21.6 \\
\hline $\begin{array}{l}\text { Grand Mesa, } \\
\text { Colo. }\end{array}$ & $3 / 28 / 2004$ & E & 2.5 & 8.1 & 5.58 & 2.6 & 27.0 & 5.0 & 1.4 & 7.8 & 6.4 & 2.6 & 10.9 & 13.9 & 2.2 & 37 & 101 & na & 5.3 & 25.3 \\
\hline $\begin{array}{l}\text { Granite Pass, } \\
\text { Mont. }\end{array}$ & 3/7/2004 & E & -15.1 & 5.4 & 4.92 & 12.0 & $<3.1$ & $<1.7$ & 1.3 & 1.1 & 2.1 & 1.3 & 2.0 & 2.8 & 0.7 & 64 & 222 & na & 6.3 & 45.7 \\
\hline $\begin{array}{l}\text { Gypsum Creek, } \\
\text { Wyo. }\end{array}$ & $3 / 23 / 2004$ & E & -7.1 & 4.3 & 5.35 & 4.5 & 4.6 & $<1.7$ & $<1.0$ & 0.4 & 2.5 & 1.3 & 3.1 & 6.9 & 0.6 & 24 & 91 & na & 0.9 & 3.1 \\
\hline $\begin{array}{l}\text { Hopewell, } \\
\text { N. Mex. }\end{array}$ & $3 / 27 / 2004$ & E & -6.1 & 5.5 & 5.23 & 5.9 & 10.9 & 1.8 & $<1.0$ & 2.1 & 4.5 & 1.3 & 6.9 & 11.3 & 1.0 & 34 & 87 & na & 4.1 & 12.9 \\
\hline $\begin{array}{l}\text { Kings Hill, } \\
\text { Mont. }\end{array}$ & $2 / 28 / 2004$ & E & -10.2 & 3.8 & 5.11 & 7.8 & 4.2 & $<1.7$ & $<1.0$ & 1.1 & 4.8 & $<1.0$ & 4.1 & 7.1 & 0.8 & 42 & 128 & 8.0 & 3.0 & 23.0 \\
\hline Lake, Wyo. & $2 / 25 / 2004$ & E & -5.5 & 3.5 & 5.42 & 3.8 & $<3.1$ & $<1.7$ & 1.2 & $<0.4$ & 5.1 & $<1.0$ & 2.8 & 5.7 & 0.5 & 28 & 105 & na & 2.1 & 8.6 \\
\hline $\begin{array}{l}\text { Lake Irene For- } \\
\text { est, Colo. }\end{array}$ & $3 / 30 / 2004$ & E & -8.4 & 6.1 & 5.12 & 7.6 & 9.3 & $<1.7$ & $<1.0$ & 1.8 & 5.0 & $<1.0$ & 7.2 & 12.4 & 1.0 & 44 & 145 & na & 2.6 & 9.3 \\
\hline $\begin{array}{l}\text { Lake Irene } \\
\text { Meadow, } \\
\text { Colo. }\end{array}$ & $3 / 30 / 2004$ & E & -7.8 & 3.7 & 5.23 & 5.9 & $<3.1$ & $<1.7$ & $<1.0$ & $<0.4$ & 3.5 & $<1.0$ & 2.6 & 8.2 & $<0.4$ & 35 & 105 & na & 1.4 & -7.2 \\
\hline $\begin{array}{c}\text { Lewis Lake } \\
\text { Divide, } \\
\text { Wyo. }\end{array}$ & $2 / 23 / 2004$ & E & -1.7 & 3.7 & 5.54 & 2.9 & 4.4 & $<1.7$ & 3.0 & $<0.4$ & 4.9 & $<1.0$ & 5.2 & 5.7 & $<0.4$ & 80 & 235 & na & 1.9 & 16.5 \\
\hline $\begin{array}{l}\text { Lionshead, } \\
\text { Mont. }\end{array}$ & $3 / 1 / 2004$ & E & -10.9 & 7.3 & 5.04 & 9.1 & 4.1 & $<1.7$ & 1.8 & 2.0 & 9.6 & 1.6 & 7.6 & 10.0 & 0.9 & na & 170 & na & 2.1 & 16.2 \\
\hline $\begin{array}{l}\text { Loch Vale For- } \\
\text { est, Colo. }\end{array}$ & 4/6/2004 & E & -11.9 & 6.8 & 5.20 & 6.3 & 9.8 & $<1.7$ & $<1.0$ & 2.1 & 9.3 & $<1.0$ & 8.0 & 14.4 & 0.7 & 73 & 219 & 4.5 & 3.9 & 10.4 \\
\hline $\begin{array}{l}\text { Loch Vale } \\
\text { Meadow, } \\
\text { Colo. }\end{array}$ & $4 / 6 / 2004$ & E & -5.4 & 4.9 & 5.29 & 5.1 & 7.1 & $<1.7$ & $<1.0$ & 0.9 & 5.7 & $<1.0$ & 5.8 & 11.5 & 0.4 & 110 & 302 & na & 2.0 & 4.2 \\
\hline $\begin{array}{l}\text { Loveland Pass, } \\
\text { Colo. }\end{array}$ & $4 / 9 / 2004$ & E & -13.1 & 4.8 & 5.17 & 6.8 & 3.7 & $<1.7$ & 1.1 & $<0.4$ & 5.2 & 2.3 & 3.8 & 8.4 & 0.4 & 42 & 144 & na & 0.8 & 7.6 \\
\hline $\begin{array}{l}\text { Lynx Pass, } \\
\text { Colo. }\end{array}$ & $3 / 26 / 2004$ & E & -10.2 & 4.7 & 5.40 & 4.0 & 11.1 & $<1.7$ & $<1.0$ & 1.1 & 2.8 & $<1.0$ & 3.6 & 11.8 & $<0.4$ & 29 & 80 & na & 0.9 & 10.5 \\
\hline $\begin{array}{l}\text { Mills Lake, } \\
\text { Colo. }\end{array}$ & $3 / 24 / 2004$ & E & -7.8 & 6.7 & 5.25 & 5.6 & 11.6 & 1.7 & $<1.0$ & 0.5 & 8.6 & $<1.0$ & 8.7 & 16.3 & 0.8 & 58 & 170 & na & 2.5 & 5.7 \\
\hline
\end{tabular}


Table 13. Selected chemical and physical data for Rocky Mountain snowpack samples for 2004.—Continued

[E, depth-integrated environmental; ANC, acid neutralization capacity; $\mu$ eq/L, microequivalents per liter; $\mu \mathrm{S} / \mathrm{cm}$, microsiemens per centimeter; $\mathrm{SC}$, specific conductance at $25^{\circ} \mathrm{C}$; $\mathrm{pH}$ values in standard units; $\mathrm{H}$, hydrogen; Ca, calcium; Mg, magnesium; $\mathrm{Na}$, sodium; $\mathrm{K}$, potassium; $\mathrm{NH}_{4}$, ammonium; $\mathrm{Cl}$, chloride; $\mathrm{SO}_{4}$, sulfate; $\mathrm{NO}_{3}$, nitrate; DOC, dissolved organic carbon; mg/L, milligrams per liter; $\mathrm{SWE}$, snow/water equivalent; cm, centimeters; NADP, National Atmospheric Deposition Program; $\delta^{34} \mathrm{~S}$, stable sulfur isotope ratio $\left.{ }^{34} \mathrm{~S} /{ }^{2} \mathrm{~S}\right) ; \mathrm{Hg}$, mercury; ng/L, nanograms per liter; na, not analyzed; <, below reporting limits; (), suspect ionic balance\%, percent]

\begin{tabular}{|c|c|c|c|c|c|c|c|c|c|c|c|c|c|c|c|c|c|c|c|c|}
\hline Site name & $\begin{array}{l}\text { Sample } \\
\text { date }\end{array}$ & $\begin{array}{l}\text { Sample } \\
\text { type }\end{array}$ & $\begin{array}{c}\text { ANC } \\
(\mu \mathrm{eq} / \mathrm{L})\end{array}$ & $\begin{array}{c}\mathrm{SC} \\
(\mu \mathrm{S} / \mathrm{cm})\end{array}$ & $\mathrm{pH}$ & $\underset{(\mu e q / L)}{H}$ & 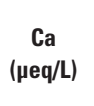 & $\underset{(\mu \mathrm{eq} / \mathrm{L})}{\mathrm{Mg}}$ & $\underset{(\mu \mathrm{Na} / \mathrm{L})}{\mathrm{Na}}$ & $\begin{array}{c}K \\
\text { ( } \mu \mathrm{eq} / L \text { ) }\end{array}$ & 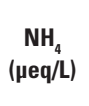 & $\underset{(\mu \mathrm{eq} / \mathrm{L})}{\mathrm{Cl}}$ & $\underset{(\mu \mathrm{eq} / \mathrm{L})}{\mathrm{SO}_{4}}$ & $\begin{array}{c}\mathrm{NO}_{3} \\
(\mu \mathrm{eq} / \mathrm{L})\end{array}$ & $\begin{array}{l}\text { DOC } \\
\text { (mg/L) }\end{array}$ & $\begin{array}{l}\text { SWE } \\
\text { (cm) }\end{array}$ & $\begin{array}{l}\text { Snow } \\
\text { depth } \\
\text { (cm) }\end{array}$ & $\begin{array}{c}\delta^{34} \mathbf{S} \\
\text { (per mil) }\end{array}$ & $\underset{(n g / L)}{\mathrm{Hg}}$ & $\begin{array}{c}\text { lonic } \\
\text { balance } \\
(\%)\end{array}$ \\
\hline $\begin{array}{l}\text { Molas Lake, } \\
\text { Colo. }\end{array}$ & $3 / 27 / 2004$ & $\mathrm{E}$ & -2.7 & 5.2 & 5.51 & 3.1 & 9.3 & $<1.7$ & 3.8 & 1.6 & 3.6 & 4.5 & 4.9 & 9.1 & 1.1 & 37 & 99 & 3.5 & 1.1 & 7.4 \\
\hline $\begin{array}{l}\text { Monarch Pass, } \\
\text { Colo. }\end{array}$ & $3 / 25 / 2004$ & E & -14.0 & 4.5 & 4.83 & 14.8 & 11.2 & 3.1 & $<1.0$ & 6.1 & 4.0 & 2.1 & 4.7 & 7.8 & 2.0 & 31 & 82 & na & 5.7 & $(45.4)$ \\
\hline $\begin{array}{l}\text { Monida Pass, } \\
\text { Mont. }\end{array}$ & 2/26/2004 & E & -4.8 & 4.6 & 5.32 & 4.8 & 4.2 & $<1.7$ & 2.4 & $<0.4$ & 4.4 & 1.5 & 4.3 & 7.5 & $<0.4$ & 15 & 57 & na & 0.9 & 8.7 \\
\hline $\begin{array}{l}\text { Mount Bel- } \\
\text { mont, Mont. }\end{array}$ & $2 / 27 / 2004$ & E & -5.5 & 5.5 & 5.27 & 5.4 & 7.3 & 1.9 & 3.0 & 3.2 & 5.9 & 2.5 & 5.1 & 8.3 & 1.8 & 22 & 68 & na & 3.9 & 25.3 \\
\hline $\begin{array}{l}\text { Ned Wilson, } \\
\text { Colo. }\end{array}$ & 4/13/2004 & E & -7.1 & 3.5 & 5.40 & 4.0 & 7.9 & $<1.7$ & $<1.0$ & $<0.4$ & 3.7 & $<1.0$ & 3.7 & 7.2 & $<0.4$ & 76 & 224 & na & 2.0 & 18.0 \\
\hline $\begin{array}{l}\text { Niwot Snotel, } \\
\text { Colo. }\end{array}$ & $3 / 16 / 2004$ & E & -9.0 & 7.5 & 5.15 & 7.1 & 7.2 & $<1.7$ & $<1.0$ & 1.8 & 12.7 & 1.1 & 9.1 & 18.0 & 0.6 & 23 & 88 & na & 1.1 & 0.9 \\
\hline $\begin{array}{l}\text { Noisy Basin, } \\
\text { Mont. }\end{array}$ & $3 / 4 / 2004$ & E & -7.0 & 4.4 & 5.10 & 7.9 & $<3.1$ & $<1.7$ & $<1.0$ & $<0.4$ & 3.1 & $<1.0$ & 3.2 & 5.5 & $<0.4$ & 82 & 303 & na & 1.5 & 11.6 \\
\hline $\begin{array}{c}\text { Old Battle, } \\
\text { Wyo. }\end{array}$ & $3 / 25 / 2004$ & E & -1.2 & 6.1 & 5.31 & 4.9 & 11.7 & 3.5 & $<1.0$ & 2.7 & 5.0 & 1.7 & 9.9 & 14.3 & 1.4 & 74 & 197 & na & 5.4 & 3.6 \\
\hline $\begin{array}{l}\text { Old Faithful } \\
\text { Fire Road, } \\
\text { Wyo. }\end{array}$ & $2 / 26 / 2004$ & E & 1.4 & 3.4 & 5.63 & 2.3 & 4.3 & $<1.7$ & 1.5 & $<0.4$ & 5.6 & 1.6 & 3.5 & 6.3 & 0.5 & 37 & 123 & na & 1.1 & 3.3 \\
\hline $\begin{array}{l}\text { Old Faithful (in } \\
\text { road), Wyo. }\end{array}$ & $2 / 26 / 2004$ & E & -2.6 & 6.7 & 5.96 & 1.1 & 21.1 & 2.7 & 3.2 & 1.7 & 8.6 & 4.9 & 7.0 & 8.1 & 1.4 & 27 & 62 & na & 4.5 & $(31.6)$ \\
\hline $\begin{array}{l}\text { Oldman Lake, } \\
\text { Mont. }\end{array}$ & $3 / 11 / 2004$ & E & -9.2 & 4.4 & 5.08 & 8.3 & $<3.1$ & $<1.7$ & $<1.0$ & 1.9 & 1.6 & $<1.0$ & 2.7 & 4.9 & 0.9 & 68 & 190 & na & 2.0 & 22.3 \\
\hline $\begin{array}{c}\text { Phantom Val- } \\
\text { ley, Colo. }\end{array}$ & 3/7/2004 & E & -13.7 & 7.8 & 4.96 & 11.0 & 7.7 & $<1.7$ & $<1.0$ & 1.5 & 5.5 & $<1.0$ & 8.7 & 16.3 & 0.8 & 17 & 71 & na & 2.7 & 1.3 \\
\hline $\begin{array}{l}\text { Rabbit Ears 1, } \\
\text { Colo. }\end{array}$ & $3 / 31 / 2004$ & E & -16.9 & 7.2 & 4.90 & 12.6 & 4.2 & $<1.7$ & $<1.0$ & 0.7 & 4.9 & $<1.0$ & 7.9 & 13.0 & $<0.4$ & 76 & 214 & na & 0.9 & 3.7 \\
\hline $\begin{array}{l}\text { Red Mountain, } \\
\text { Mont. }\end{array}$ & $3 / 3 / 2004$ & E & -10.8 & 6.0 & 5.05 & 8.9 & 5.6 & $<1.7$ & 2.6 & 0.4 & 6.0 & 1.6 & 5.5 & 6.4 & 0.6 & 35 & 136 & na & 1.5 & 27.2 \\
\hline $\begin{array}{r}\text { Red Mountain } \\
\text { Pass, Colo. }\end{array}$ & 3/27/2004 & E & 1.3 & 4.0 & 5.79 & 1.6 & 9.2 & $<1.7$ & 4.1 & 0.4 & 3.2 & 4.3 & 3.5 & 6.5 & $<0.4$ & 49 & 140 & na & 1.3 & 8.8 \\
\hline $\begin{array}{l}\text { Rendezvous } \\
\text { Mountain, } \\
\text { Wyo. }\end{array}$ & $3 / 24 / 2004$ & E & -5.5 & 3.9 & 5.48 & 3.3 & $<3.1$ & $<1.7$ & 1.2 & 0.5 & 4.5 & $<1.0$ & 3.8 & 4.5 & $<0.4$ & 90 & 243 & na & 0.7 & 6.1 \\
\hline $\begin{array}{l}\text { Ripple Creek } \\
\text { NADP, } \\
\text { Colo. }\end{array}$ & $4 / 1 / 2004$ & E & -8.3 & 4.9 & 5.42 & 3.8 & 8.6 & $<1.7$ & $<1.0$ & 0.6 & 4.3 & $<1.0$ & 4.7 & 10.9 & $<0.4$ & 83 & 220 & na & 1.6 & 5.2 \\
\hline
\end{tabular}


[E, depth-integrated environmental; ANC, acid neutralization capacity; $\mu \mathrm{eq} / \mathrm{L}$, microequivalents per liter; $\mu \mathrm{S} / \mathrm{cm}$, microsiemens per centimeter; $\mathrm{SC}$, specific conductance at $25^{\circ} \mathrm{C} ; \mathrm{pH}$ values in standard units; $\mathrm{H}$, hydrogen; $\mathrm{Ca}$, calcium; $\mathrm{Mg}$, magnesium; $\mathrm{Na}$, sodium; $\mathrm{K}$, potassium; $\mathrm{NH}_{4}$, ammonium; $\mathrm{Cl}$, chloride; $\mathrm{SO}_{4}$, sulfate; $\mathrm{NO}_{3}$, nitrate; DOC, dissolved organic carbon; mg/L, milligrams per liter; $\mathrm{SWE}$, snow/water equivalent; cm, centimeters; NADP, National Atmospheric Deposition Program; $\delta^{34} \mathrm{~S}$, stable sulfur isotope ratio $\left({ }^{34} \mathrm{~S} /{ }^{22} \mathrm{~S}\right) ; \mathrm{Hg}$, mercury; ng/L, nanograms per liter; na, not analyzed; <, below reporting limits; (), suspect ionic balance\%, percent]

\begin{tabular}{|c|c|c|c|c|c|c|c|c|c|c|c|c|c|c|c|c|c|c|c|c|}
\hline Site name & $\begin{array}{l}\text { Sample } \\
\text { date }\end{array}$ & $\begin{array}{c}\text { Sample } \\
\text { type }\end{array}$ & $\begin{array}{c}\text { ANC } \\
\text { ( } \mu \mathrm{eq} / \mathrm{L})\end{array}$ & $\begin{array}{c}\mathrm{SC} \\
(\mu \mathrm{S} / \mathrm{cm})\end{array}$ & pH & 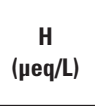 & $\begin{array}{c}\text { Ca } \\
(\mu \mathrm{eq} / \mathrm{L})\end{array}$ & $\begin{array}{c}\mathrm{Mg} \\
(\mu \mathrm{eq} / \mathrm{L})\end{array}$ & $\underset{(\mu \mathrm{Na} / \mathrm{L})}{\mathrm{Na}}$ & $\begin{array}{c}K \\
\text { ( } \mu \text { eq/L) }\end{array}$ & $\begin{array}{c}\mathrm{NH}_{4} \\
(\mu \mathrm{eq} / \mathrm{L})\end{array}$ & $\underset{(\mu e q / L)}{C l}$ & $\begin{array}{c}\mathrm{SO}_{4} \\
(\mu \mathrm{eq} / \mathrm{L})\end{array}$ & 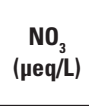 & $\begin{array}{l}\text { DOC } \\
\text { (mg/L) }\end{array}$ & $\begin{array}{l}\text { SWE } \\
(\mathrm{cm})\end{array}$ & $\begin{array}{l}\text { Snow } \\
\text { depth } \\
(\mathrm{cm})\end{array}$ & $\begin{array}{c}\delta^{34} \mathrm{~S} \\
\text { (per mil) }\end{array}$ & $\underset{\text { (ng/L) }}{\mathrm{Hg}}$ & $\begin{array}{c}\text { lonic } \\
\text { balance } \\
(\%)\end{array}$ \\
\hline $\begin{array}{l}\text { Slumgullion } \\
\text { Pass, Colo. }\end{array}$ & $3 / 26 / 2004$ & E & -11.5 & 4.4 & 5.02 & 9.5 & 7.9 & 2.8 & $<1.0$ & 6.4 & 3.3 & 1.4 & 4.8 & 7.4 & 2.2 & 19 & 81 & 5.0 & 3.8 & 37.9 \\
\hline $\begin{array}{l}\text { Snow Bowl, } \\
\text { Mont. }\end{array}$ & $3 / 6 / 2004$ & E & -24.4 & 3.2 & 4.73 & 18.6 & $<3.1$ & $<1.7$ & $<1.0$ & 0.9 & 1.6 & $<1.0$ & 2.4 & 3.4 & 0.8 & 57 & 198 & na & 3.6 & 56.9 \\
\hline $\begin{array}{l}\text { Snyder Lake } \\
\text { (Glacier } \\
\text { NP), Mont. }\end{array}$ & $3 / 8 / 2004$ & E & -5.4 & 3.9 & 5.26 & 5.5 & $<3.1$ & $<1.7$ & 1.5 & 0.5 & 2.9 & 1.5 & 2.7 & 5.3 & 0.5 & 43 & 137 & na & 3.2 & 4.7 \\
\hline $\begin{array}{l}\text { South Pass, } \\
\text { Wyo. }\end{array}$ & $3 / 29 / 2004$ & E & -10.0 & 5.9 & 5.16 & 6.9 & 7.0 & $<1.7$ & $<1.0$ & 1.8 & 4.6 & $<1.0$ & 8.0 & 8.4 & 0.9 & 41 & 122 & na & 1.8 & 10.7 \\
\hline $\begin{array}{l}\text { Spring Gulch, } \\
\text { Mont. }\end{array}$ & $2 / 27 / 2004$ & E & -14.3 & 5.2 & 4.95 & 11.2 & 4.4 & $<1.7$ & $<1.0$ & 3.3 & 8.7 & $<1.0$ & 6.5 & 9.5 & 1.3 & 22 & 68 & na & 2.0 & 26.5 \\
\hline $\begin{array}{l}\text { Sunlight Peak, } \\
\text { Colo. }\end{array}$ & 4/2/2004 & E & -6.7 & 5.8 & 5.26 & 5.5 & 18.5 & 3.9 & $<1.0$ & 5.5 & 6.4 & 1.6 & 8.9 & 14.5 & 2.3 & 39 & 133 & na & 4.7 & 22.8 \\
\hline $\begin{array}{l}\text { Sylvan Lake, } \\
\text { Wyo. }\end{array}$ & $2 / 24 / 2004$ & E & -6.6 & 3.6 & 5.42 & 3.8 & $<3.1$ & $<1.7$ & $<1.0$ & 0.5 & 4.0 & $<1.0$ & 3.1 & 5.0 & 0.5 & 46 & 142 & na & 1.3 & 0.9 \\
\hline $\begin{array}{l}\text { Sylvan Lake (in } \\
\text { road), Wyo. }\end{array}$ & $2 / 24 / 2004$ & E & -6.5 & 4.0 & 5.32 & 4.8 & $<3.1$ & $<1.7$ & 1.5 & 0.5 & 4.9 & $<1.0$ & 3.9 & 6.4 & $<0.4$ & 44 & 104 & na & 1.9 & 5.8 \\
\hline $\begin{array}{l}\text { Taos Ski Val- } \\
\text { ley, N. Mex. }\end{array}$ & $3 / 28 / 2004$ & E & -12.2 & 3.6 & 5.05 & 8.9 & 4.2 & $<1.7$ & $<1.0$ & 0.9 & 4.3 & $<1.0$ & 3.8 & 6.6 & 0.7 & 36 & 115 & 4.1 & 7.5 & 27.8 \\
\hline $\begin{array}{c}\text { Teton Pass, } \\
\text { Wyo. }\end{array}$ & $3 / 19 / 2004$ & E & 6.7 & 4.7 & 6.03 & 0.9 & 8.6 & 2.9 & 1.6 & 2.0 & 5.6 & 1.4 & 4.4 & 6.5 & 0.7 & 48 & 199 & na & 2.5 & 6.4 \\
\hline $\begin{array}{l}\text { Togwotee Pass, } \\
\text { Wyo. }\end{array}$ & $3 / 18 / 2004$ & E & -0.5 & 3.1 & 5.69 & 2.0 & 4.6 & $<1.7$ & 1.7 & 0.8 & 3.4 & $<1.0$ & 3.6 & 5.0 & 0.4 & 40 & 138 & na & 1.5 & 18.8 \\
\hline $\begin{array}{l}\text { Twenty-one } \\
\text { Mile, Mont. }\end{array}$ & $3 / 1 / 2004$ & E & -12.2 & 6.7 & 5.06 & 8.7 & 6.0 & $<1.7$ & 1.2 & 2.8 & 5.5 & 1.4 & 4.3 & 7.4 & 1.3 & 36 & 134 & na & 1.4 & 29.9 \\
\hline $\begin{array}{l}\text { University } \\
\text { Camp, Colo. }\end{array}$ & $4 / 5 / 2004$ & E & -8.8 & 6.4 & 5.21 & 6.2 & 5.9 & $<1.7$ & $<1.0$ & 1.0 & 9.0 & $<1.0$ & 7.9 & 12.7 & 0.5 & 60 & 184 & na & 0.8 & 3.7 \\
\hline $\begin{array}{l}\text { West Yellow- } \\
\text { stone, Mont. }\end{array}$ & 2/26/2004 & E & -2.8 & 5.6 & 5.20 & 6.3 & 5.0 & $<1.7$ & 1.8 & 0.8 & 7.5 & 1.4 & 4.8 & 11.7 & 1.0 & 24 & 90 & na & 1.4 & 9.1 \\
\hline $\begin{array}{l}\text { West Yellow- } \\
\text { stone (in } \\
\text { road), Mont. }\end{array}$ & $2 / 26 / 2004$ & E & -4.6 & 5.5 & 5.37 & 4.3 & 3.3 & $<1.7$ & 4.6 & 0.9 & 8.9 & 5.5 & 5.2 & 8.2 & 0.8 & 38 & 83 & na & 1.1 & 7.7 \\
\hline $\begin{array}{l}\text { Wolf Creek } \\
\text { Pass, Colo. }\end{array}$ & $3 / 26 / 2004$ & E & -3.6 & 5.9 & 5.27 & 5.4 & 7.1 & 2.0 & $<1.0$ & 5.2 & 7.9 & 2.0 & 9.2 & 11.4 & 1.4 & 68 & 187 & na & 4.3 & 10.1 \\
\hline
\end{tabular}


Table 14. Selected chemical and physical data for Rocky Mountain snowpack samples for 2005.

[E, depth-integrated environmental; ANC, acid neutralization capacity; $\mu$ eq/L, microequivalents per liter; $\mu \mathrm{S} / \mathrm{cm}$, microsiemens per centimeter; $\mathrm{SC}$, specific conductance at $25^{\circ} \mathrm{C} ; \mathrm{pH}$ values in standard units; $\mathrm{H}$, hydrogen; $\mathrm{Ca}$, calcium; $\mathrm{Mg}$, magnesium; $\mathrm{Na}$, sodium; $\mathrm{K}$, potassium; $\mathrm{NH}_{4}$, ammonium; $\mathrm{Cl}$, chloride; $\mathrm{SO}_{4}$, sulfate; $\mathrm{NO}_{3}$, nitrate; DOC, dissolved organic carbon; mg/L, milligrams per liter; $\mathrm{SWE}$, snow/water equivalent; cm, centimeters; NADP, National Atmospheric Deposition Program; $\delta^{34} \mathrm{~S}$, stable sulfur isotope ratio $\left({ }^{34} \mathrm{~S} /{ }^{32} \mathrm{~S}\right)$; Hg, mercury; ng/L, nanograms per liter; na, not analyzed; <, below reporting limits;

$\%$, percent]

\begin{tabular}{|c|c|c|c|c|c|c|c|c|c|c|c|c|c|c|c|c|c|c|c|c|}
\hline Site name & $\begin{array}{l}\text { Sample } \\
\text { date }\end{array}$ & $\begin{array}{l}\text { Sample } \\
\text { type }\end{array}$ & $\begin{array}{c}\text { ANC } \\
\text { ( } \mu \mathrm{eq} / \mathrm{L})\end{array}$ & $\begin{array}{c}S C \\
(\mu \mathrm{S} / \mathrm{cm})\end{array}$ & $\mathrm{pH}$ & $\begin{array}{c}\text { H } \\
(\mu \mathrm{eq} / \mathrm{L})\end{array}$ & $\begin{array}{c}\mathrm{Ca} \\
(\mu \mathrm{eq} / \mathrm{L})\end{array}$ & $\underset{(\mu \mathrm{eq} / \mathrm{L})}{\mathrm{Mg}}$ & $\begin{array}{c}\mathrm{Na} \\
(\mu \mathrm{eq} / \mathrm{L})\end{array}$ & $\begin{array}{c}K \\
(\mu \mathrm{eq} / \mathrm{L})\end{array}$ & $\begin{array}{c}\mathrm{NH}_{4} \\
(\mu \mathrm{eq} / \mathrm{L})\end{array}$ & $\underset{(\mu \mathrm{eq} / \mathrm{L})}{\mathrm{Cl}}$ & $\begin{array}{c}\mathrm{SO}_{4} \\
(\mu \mathrm{eq} / \mathrm{L})\end{array}$ & $\begin{array}{c}\mathrm{NO}_{3} \\
(\mu \mathrm{eq} / \mathrm{L})\end{array}$ & $\begin{array}{l}\text { DOC } \\
\text { (mg/L) }\end{array}$ & $\begin{array}{l}\text { SWE } \\
\text { (cm) }\end{array}$ & $\begin{array}{l}\text { Snow } \\
\text { depth } \\
(\mathrm{cm})\end{array}$ & $\begin{array}{c}\delta^{34} \mathbf{S} \\
\text { (per mil) }\end{array}$ & $\underset{(n g / L)}{\mathrm{Hg}}$ & $\begin{array}{c}\text { lonic } \\
\text { balance } \\
(\%)\end{array}$ \\
\hline $\begin{array}{l}\text { Banner Summit, } \\
\text { Idaho }\end{array}$ & $3 / 10 / 2005$ & E & -7.2 & 4.3 & 5.25 & 5.6 & $<3.1$ & $<1.7$ & $<1.0$ & $<0.4$ & 2.8 & $<1.0$ & 2.6 & 4.1 & $<0.4$ & 32 & 109 & 5.1 & 1.3 & 11.6 \\
\hline $\begin{array}{l}\text { Berthoud Pass, } \\
\text { Colo. }\end{array}$ & $4 / 4 / 2005$ & E & -6.7 & 6.6 & 5.18 & 6.6 & 7.5 & $<1.7$ & 5.1 & 1.0 & 5.7 & 4.0 & 6.0 & 11.2 & 0.4 & 63 & 204 & na & 4.2 & 9.9 \\
\hline $\begin{array}{l}\text { Big Mountain, } \\
\text { Mont. }\end{array}$ & $3 / 5 / 2005$ & E & -10.8 & 5.4 & 5.09 & 8.1 & $<3.1$ & $<1.7$ & $<1.0$ & $<0.4$ & 3.0 & 1.0 & 3.6 & 5.5 & $<0.4$ & 53 & 160 & 4.2 & 1.6 & 4.8 \\
\hline Big Sky, Mont. & 3/9/2005 & E & 1.7 & 4.5 & 5.52 & 3.0 & 9.0 & $<1.7$ & $<1.0$ & 0.7 & 4.7 & 2.4 & 3.2 & 6.0 & $<0.4$ & 18 & 59 & na & 1.7 & 13.2 \\
\hline $\begin{array}{l}\text { Brooklyn Lake, } \\
\text { Wyo. }\end{array}$ & $3 / 31 / 2005$ & E & -5.0 & 4.7 & 5.38 & 4.2 & 6.5 & $<1.7$ & 1.5 & 0.7 & 4.8 & $<1.0$ & 6.0 & 6.7 & $<0.4$ & 58 & 189 & 5.7 & 2.7 & 16.6 \\
\hline Brumley, Colo. & $3 / 21 / 2005$ & E & -6.4 & 4.7 & 5.32 & 4.8 & 5.5 & $<1.7$ & 1.3 & 0.5 & 2.7 & $<1.0$ & 3.6 & 6.8 & $<0.4$ & 30 & 130 & na & 1.9 & 17.2 \\
\hline $\begin{array}{l}\text { Buffalo Pass, } \\
\text { Colo. }\end{array}$ & $3 / 29 / 2005$ & E & -11.6 & 5.9 & 5.13 & 7.4 & 4.5 & $<1.7$ & 1.1 & 0.7 & 4.8 & $<1.0$ & 7.1 & 7.9 & $<0.4$ & 110 & 351 & 6.3 & 2.9 & 10.3 \\
\hline $\begin{array}{l}\text { Cameron Pass, } \\
\text { Colo. }\end{array}$ & 3/23/2005 & E & -5.6 & 5.2 & 5.34 & 4.6 & 7.0 & $<1.7$ & 2.1 & 2.1 & 5.6 & 1.4 & 6.4 & 7.8 & 0.6 & 51 & 189 & na & 2.3 & 15.4 \\
\hline Canyon, Wyo. & $2 / 23 / 2005$ & E & -6.5 & 4.6 & 5.29 & 5.1 & $<3.1$ & $<1.7$ & 1.2 & 0.6 & 6.9 & $<1.0$ & 2.7 & 5.9 & $<0.4$ & 21 & 90 & na & 2.4 & 23.5 \\
\hline $\begin{array}{l}\text { Chief Joseph } \\
\text { Pass, Mont. }\end{array}$ & $3 / 8 / 2005$ & E & -8.5 & 4.9 & 5.17 & 6.8 & $<3.1$ & $<1.7$ & $<1.0$ & 0.8 & 3.7 & 1.0 & 3.0 & 4.3 & 0.4 & 27 & 92 & na & 2.6 & 15.2 \\
\hline $\begin{array}{c}\text { Daisy Pass, } \\
\text { Mont. }\end{array}$ & $3 / 1 / 2005$ & E & -6.7 & 4.3 & 5.33 & 4.7 & $<3.1$ & $<1.7$ & $<1.0$ & 0.4 & 6.1 & $<1.0$ & 3.2 & 5.8 & $<0.4$ & 43 & 142 & na & 24.3 & 10.8 \\
\hline $\begin{array}{l}\text { Deadman Pass, } \\
\text { Colo. }\end{array}$ & 4/1/2005 & E & -2.1 & 5.3 & 5.44 & 3.6 & 9.5 & $<1.7$ & 5.0 & 2.3 & 4.2 & 1.2 & 7.4 & 9.2 & 0.7 & 48 & 190 & na & 6.1 & 15.9 \\
\hline $\begin{array}{l}\text { Divide Peak, } \\
\text { Wyo. }\end{array}$ & $3 / 30 / 2005$ & E & -6.8 & 6.2 & 5.24 & 5.8 & 5.5 & $<1.7$ & 2.2 & 0.5 & 5.3 & $<1.0$ & 7.4 & 9.2 & $<0.4$ & 46 & 137 & na & 1.2 & 7.2 \\
\hline Dry Lake, Colo. & $3 / 23 / 2005$ & E & -10.8 & 6.1 & 5.21 & 6.2 & 8.0 & $<1.7$ & 2.5 & 0.5 & 5.0 & $<1.0$ & 8.1 & 11.4 & $<0.4$ & 45 & 147 & 6.1 & 1.2 & 6.4 \\
\hline $\begin{array}{l}\text { Dunckley Pass, } \\
\text { Colo. }\end{array}$ & $3 / 28 / 2005$ & E & 1.6 & 5.0 & 5.54 & 2.9 & 8.5 & $<1.7$ & 1.9 & 0.8 & 5.3 & 1.1 & 5.6 & 8.4 & $<0.4$ & 46 & 168 & na & 1.4 & 7.7 \\
\hline $\begin{array}{l}\text { E5 (Glacier NP), } \\
\text { Mont. }\end{array}$ & $3 / 23 / 2005$ & E & -9.0 & 5.0 & 5.19 & 6.5 & $<3.1$ & $<1.7$ & $<1.0$ & 0.8 & 3.3 & 1.1 & 4.4 & 5.0 & 0.4 & 84 & 240 & na & 11.5 & 0.2 \\
\hline Elk River, Colo. & $3 / 24 / 2005$ & E & -3.8 & 4.9 & 5.56 & 2.8 & 12.5 & $<1.7$ & 2.4 & 1.3 & 4.3 & $<1.0$ & 7.2 & 9.8 & 0.5 & 40 & 138 & 5.4 & 2.3 & 15.5 \\
\hline $\begin{array}{l}\text { Elkhart Park, } \\
\text { Wyo. }\end{array}$ & $3 / 15 / 2005$ & E & -10.4 & 5.5 & 5.17 & 6.8 & $<3.1$ & $<1.7$ & $<1.0$ & 1.0 & 4.3 & $<1.0$ & 4.2 & 6.7 & 0.8 & 45 & 112 & na & 3.0 & 5.6 \\
\hline $\begin{array}{l}\text { Four Mile } \\
\text { Meadow, } \\
\text { Wyo. }\end{array}$ & $3 / 14 / 2005$ & E & -6.0 & 5.2 & 5.25 & 5.6 & $<3.1$ & $<1.7$ & $<1.0$ & $<0.4$ & 5.2 & $<1.0$ & 2.7 & 6.5 & $<0.4$ & 17 & 70 & na & 1.5 & 8.0 \\
\hline
\end{tabular}


[E, depth-integrated environmental; ANC, acid neutralization capacity; $\mu \mathrm{eq} / \mathrm{L}$, microequivalents per liter; $\mu \mathrm{S} / \mathrm{cm}$, microsiemens per centimeter; $\mathrm{SC}$, specific conductance at $25^{\circ} \mathrm{C}$; $\mathrm{pH}$ values in standard units; $\mathrm{H}$, hydrogen; $\mathrm{Ca}$, calcium; $\mathrm{Mg}$, magnesium; $\mathrm{Na}$, sodium; $\mathrm{K}$, potassium; $\mathrm{NH}_{4}$, ammonium; $\mathrm{Cl}$, chloride; $\mathrm{SO}_{4}$, sulfate; $\mathrm{NO}_{3}$, nitrate; DOC, dissolved organic carbon; mg/L, milligrams per liter; $\mathrm{SWE}$, snow/water equivalent; cm, centimeters; NADP, National Atmospheric Deposition Program; $\delta^{34} \mathrm{~S}$, stable sulfur isotope ratio $\left({ }^{34} \mathrm{~S} /{ }^{32} \mathrm{~S}\right)$; Hg, mercury; ng/L, nanograms per liter; na, not analyzed; <, below reporting limits; $\%$, percent]

\begin{tabular}{|c|c|c|c|c|c|c|c|c|c|c|c|c|c|c|c|c|c|c|c|c|}
\hline Site name & $\begin{array}{c}\text { Sample } \\
\text { date }\end{array}$ & $\begin{array}{c}\text { Sample } \\
\text { type }\end{array}$ & $\begin{array}{c}\text { ANC } \\
(\mu \mathrm{eq} / \mathrm{L})\end{array}$ & $\underset{(\mu \mathrm{S} / \mathrm{cm})}{\mathrm{SC}}$ & pH & 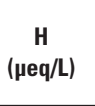 & $\begin{array}{c}\text { Ca } \\
(\mu \mathrm{eq} / \mathrm{L})\end{array}$ & $\underset{(\mu \mathrm{eg} / \mathrm{L})}{\mathbf{M g}}$ & $\begin{array}{c}\mathrm{Na} \\
(\mu \mathrm{eq} / \mathrm{L})\end{array}$ & $\underset{\text { (peq/L) }}{K}$ & $\underset{(\mu \mathrm{eq} / \mathrm{L})}{\mathrm{NH}_{4}}$ & $\begin{array}{c}C I \\
(\mu e q / L)\end{array}$ & $\underset{\text { (peq/L) }}{\mathrm{SO}_{4}}$ & $\begin{array}{c}\mathrm{NO}_{3} \\
(\mu \mathrm{eq} / \mathrm{L})\end{array}$ & $\begin{array}{c}\text { DOC } \\
\text { (mg/L) }\end{array}$ & $\begin{array}{l}\text { SWE } \\
\text { (cm) }\end{array}$ & $\begin{array}{l}\text { Snow } \\
\text { depth } \\
(\mathrm{cm})\end{array}$ & $\begin{array}{c}\delta^{34} \mathrm{~S} \\
\text { (per mil) }\end{array}$ & $\begin{array}{c}\mathrm{Hg} \\
\text { (ng/L) }\end{array}$ & $\begin{array}{c}\text { lonic } \\
\text { balance } \\
(\%)\end{array}$ \\
\hline $\begin{array}{l}\text { Fremont Pass, } \\
\text { Colo. }\end{array}$ & $4 / 6 / 2005$ & $\mathrm{E}$ & -8.0 & 5.3 & 5.20 & 6.3 & 6.0 & $<1.7$ & 1.3 & 1.1 & 3.2 & 1.0 & 3.7 & 7.5 & 0.4 & 32 & 134 & 5.2 & 2.1 & 18.7 \\
\hline $\begin{array}{l}\text { Galena Summit, } \\
\text { Idaho }\end{array}$ & $3 / 10 / 2005$ & E & -10.8 & 4.7 & 5.16 & 6.9 & $<3.1$ & $<1.7$ & $<1.0$ & $<0.4$ & 5.3 & $<1.0$ & 3.8 & 5.8 & $<0.4$ & 36 & 121 & 3.9 & 0.6 & 12.0 \\
\hline $\begin{array}{l}\text { Garnet Canyon, } \\
\text { Wyo. }\end{array}$ & $3 / 17 / 2005$ & E & 4.9 & 5.3 & 5.79 & 1.6 & 8.0 & $<1.7$ & 11.5 & 1.2 & 9.7 & 1.2 & 8.7 & 9.1 & $<0.4$ & 34 & 126 & na & 0.9 & 14.5 \\
\hline $\begin{array}{l}\text { Grand Mesa, } \\
\text { Colo. }\end{array}$ & $3 / 21 / 2005$ & E & -0.3 & 4.5 & 5.50 & 3.2 & 6.5 & $<1.7$ & $<1.0$ & 1.3 & 5.1 & $<1.0$ & 5.3 & 6.4 & 0.5 & 72 & 220 & na & 4.9 & 15.7 \\
\hline $\begin{array}{l}\text { Granite Park, } \\
\text { Mont. }\end{array}$ & $3 / 25 / 2005$ & E & -11.7 & 5.8 & 5.16 & 6.9 & $<3.1$ & $<1.7$ & 1.6 & 0.5 & 5.3 & 1.8 & 6.0 & 7.3 & $<0.4$ & 65 & 182 & na & 1.0 & -2.2 \\
\hline $\begin{array}{l}\text { Granite Pass, } \\
\text { Mont. }\end{array}$ & 3/7/2005 & $\mathrm{E}$ & -4.4 & 4.9 & 5.29 & 5.1 & $<3.1$ & $<1.7$ & $<1.0$ & 2.1 & $<1.0$ & 1.8 & 2.1 & 3.3 & 1.2 & 23 & 78 & na & 21.7 & -0.2 \\
\hline $\begin{array}{l}\text { Gypsum Creek, } \\
\text { Wyo. }\end{array}$ & $3 / 15 / 2005$ & E & -4.1 & 4.6 & 5.37 & 4.3 & $<3.1$ & $<1.7$ & $<1.0$ & 0.9 & 3.0 & $<1.0$ & 3.1 & 7.1 & $<0.4$ & 20 & 84 & na & 2.1 & -11.4 \\
\hline $\begin{array}{l}\text { Hopewell, } \\
\text { N. Mex. }\end{array}$ & $3 / 19 / 2005$ & E & -9.4 & 5.0 & 5.22 & 6.0 & 3.5 & $<1.7$ & $<1.0$ & 0.6 & 2.8 & $<1.0$ & 4.8 & 7.6 & $<0.4$ & 48 & 156 & 4.9 & 2.2 & 2.0 \\
\hline $\begin{array}{l}\text { Kings Hill, } \\
\text { Mont. }\end{array}$ & $2 / 26 / 2005$ & E & -5.5 & 5.9 & 5.24 & 5.8 & 7.0 & $<1.7$ & 2.1 & 1.7 & 6.9 & 1.8 & 5.7 & 8.9 & 0.9 & 23 & 72 & 6.4 & 8.7 & 17.7 \\
\hline $\begin{array}{l}\text { Lake Irene For- } \\
\text { est, Colo. }\end{array}$ & $4 / 5 / 2005$ & $\mathrm{E}$ & -3.4 & 4.6 & 5.32 & 4.8 & 6.5 & $<1.7$ & 1.2 & 1.1 & 5.7 & $<1.0$ & 5.7 & 9.4 & $<0.4$ & 56 & 188 & na & 4.6 & 12.1 \\
\hline $\begin{array}{l}\text { Lake Irene } \\
\text { Meadow, } \\
\text { Colo. }\end{array}$ & $4 / 5 / 2005$ & E & -12.0 & 4.9 & 5.25 & 5.6 & $<3.1$ & $<1.7$ & 1.2 & 0.4 & 4.6 & $<1.0$ & 4.3 & 8.6 & $<0.4$ & 43 & 143 & na & 0.8 & -4.6 \\
\hline $\begin{array}{l}\text { Lewis Lake Di- } \\
\text { vide, Wyo. }\end{array}$ & $2 / 21 / 2005$ & $\mathrm{E}$ & -4.0 & 4.0 & 5.44 & 3.6 & $<3.1$ & $<1.7$ & $<1.0$ & $<0.4$ & 8.2 & $<1.0$ & 3.9 & 6.2 & $<0.4$ & 61 & 211 & na & 1.0 & 7.8 \\
\hline $\begin{array}{l}\text { Lionshead, } \\
\text { Mont. }\end{array}$ & 3/9/2005 & E & 0.0 & 6.4 & 5.28 & 5.2 & 3.5 & $<1.7$ & $<1.0$ & 1.7 & 12.2 & $<1.0$ & 6.0 & 9.8 & 0.6 & 38 & 122 & na & 4.0 & 17.9 \\
\hline $\begin{array}{l}\text { Loch Vale For- } \\
\text { est, Colo. }\end{array}$ & $4 / 7 / 2005$ & E & -7.7 & 5.9 & 5.25 & 5.6 & 5.5 & $<1.7$ & 1.1 & 0.9 & 6.3 & $<1.0$ & 6.8 & 10.9 & 0.4 & 81 & 242 & 4.7 & 3.7 & 4.7 \\
\hline $\begin{array}{l}\text { Loch Vale } \\
\text { Meadow, } \\
\text { Colo. }\end{array}$ & $4 / 7 / 2005$ & E & -4.6 & 5.0 & 5.26 & 5.5 & 3.5 & $<1.7$ & $<1.0$ & $<0.4$ & 4.3 & $<1.0$ & 4.7 & 8.3 & $<0.4$ & 56 & 153 & na & 0.7 & 1.2 \\
\hline $\begin{array}{l}\text { Loveland Pass, } \\
\text { Colo. }\end{array}$ & $4 / 9 / 2005$ & E & -5.0 & 4.6 & 5.33 & 4.7 & 4.5 & $<1.7$ & 2.8 & 0.5 & 3.3 & 3.5 & 3.1 & 7.2 & 0.4 & 50 & 160 & 5.5 & 1.1 & 6.6 \\
\hline Lynx Pass, Colo. & $3 / 18 / 2005$ & $\mathrm{E}$ & -9.7 & 4.4 & 5.34 & 4.6 & 7.0 & $<1.7$ & 2.2 & $<0.4$ & 3.2 & 1.1 & 5.7 & 9.5 & $<0.4$ & 27 & 120 & na & 0.9 & 1.7 \\
\hline
\end{tabular}


Table 14. Selected chemical and physical data for Rocky Mountain snowpack samples for 2005.—Continued

[E, depth-integrated environmental; ANC, acid neutralization capacity; $\mu$ eq/L, microequivalents per liter; $\mu \mathrm{S} / \mathrm{cm}$, microsiemens per centimeter; $\mathrm{SC}$, specific conductance at $25^{\circ} \mathrm{C} ; \mathrm{pH}$ values in standard units; $\mathrm{H}$, hydrogen; Ca, calcium; Mg, magnesium; $\mathrm{Na}$, sodium; $\mathrm{K}$, potassium; $\mathrm{NH}_{4}$, ammonium; $\mathrm{Cl}$, chloride; $\mathrm{SO}_{4}$, sulfate; $\mathrm{NO}_{3}$, nitrate; DOC, dissolved organic carbon; mg/L, milligrams per liter; $\mathrm{SWE}$, snow/water equivalent; cm, centimeters; NADP, National Atmospheric Deposition Program; $\delta^{34} \mathrm{~S}$, stable sulfur isotope ratio $\left({ }^{34} \mathrm{~S} /{ }^{32} \mathrm{~S}\right)$; Hg, mercury; ng/L, nanograms per liter; na, not analyzed; <, below reporting limits;

$\%$, percent]

\begin{tabular}{|c|c|c|c|c|c|c|c|c|c|c|c|c|c|c|c|c|c|c|c|c|}
\hline Site name & $\begin{array}{l}\text { Sample } \\
\text { date }\end{array}$ & $\begin{array}{l}\text { Sample } \\
\text { type }\end{array}$ & $\begin{array}{c}\text { ANC } \\
\text { (peq/L) }\end{array}$ & $\begin{array}{c}\text { SC } \\
(\mu \mathrm{S} / \mathrm{cm})\end{array}$ & $\mathrm{pH}$ & $\begin{array}{c}\text { H } \\
(\mu \mathrm{eq} / \mathrm{L})\end{array}$ & $\begin{array}{c}\mathrm{Ca} \\
(\mu \mathrm{eq} / \mathrm{L})\end{array}$ & $\underset{(\mu \mathrm{eq} / L)}{\mathrm{Mg}}$ & $\begin{array}{c}\mathrm{Na} \\
(\mu \mathrm{eq} / \mathrm{L})\end{array}$ & 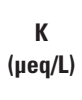 & $\begin{array}{c}\mathrm{NH}_{4} \\
(\mu \mathrm{eq} / \mathrm{L})\end{array}$ & $\begin{array}{c}C l \\
(\mu \mathrm{eq} / \mathrm{L})\end{array}$ & $\begin{array}{c}\mathrm{SO}_{4} \\
(\mu \mathrm{eq} / \mathrm{L})\end{array}$ & $\begin{array}{c}\mathrm{NO}_{3} \\
(\mu \mathrm{eq} / \mathrm{L})\end{array}$ & $\begin{array}{l}\text { DOC } \\
\text { (mg/L) }\end{array}$ & $\begin{array}{l}\text { SWE } \\
\text { (cm) }\end{array}$ & $\begin{array}{l}\text { Snow } \\
\text { depth } \\
(\mathrm{cm})\end{array}$ & $\begin{array}{c}\delta^{34} \mathbf{S} \\
\text { (per mil) }\end{array}$ & $\underset{(n g / L)}{\mathrm{Hg}}$ & $\begin{array}{c}\text { lonic } \\
\text { balance } \\
(\%)\end{array}$ \\
\hline $\begin{array}{l}\text { Mills Lake, } \\
\text { Colo. }\end{array}$ & $4 / 7 / 2005$ & E & -1.9 & 5.7 & 5.47 & 3.4 & 9.0 & $<1.7$ & 1.9 & 2.3 & 7.3 & 1.1 & 9.0 & 12.3 & 0.7 & 61 & 180 & na & na & 3.3 \\
\hline $\begin{array}{l}\text { Molas Lake, } \\
\text { Colo. }\end{array}$ & $3 / 22 / 2005$ & E & 1.1 & 4.7 & 5.24 & 5.8 & 3.5 & $<1.7$ & 1.2 & 0.6 & 2.5 & $<1.0$ & 3.4 & 6.2 & $<0.4$ & 67 & 197 & 4.0 & 1.1 & 11.9 \\
\hline $\begin{array}{l}\text { Monarch Pass, } \\
\text { Colo. }\end{array}$ & $3 / 24 / 2005$ & E & 2.9 & 4.2 & 5.93 & 1.2 & 16.0 & 2.5 & 1.7 & 0.7 & 4.1 & 1.5 & 4.9 & 7.3 & $<0.4$ & 48 & 157 & na & 1.6 & 22.3 \\
\hline $\begin{array}{l}\text { Monida Pass, } \\
\text { Mont. }\end{array}$ & $3 / 24 / 2005$ & E & -8.9 & 5.9 & 5.42 & 3.8 & 3.5 & $<1.7$ & $<1.0$ & $<0.4$ & 14.7 & $<1.0$ & 7.5 & 12.9 & $<0.4$ & 9 & 32 & na & 0.9 & 3.9 \\
\hline $\begin{array}{l}\text { Mount Belmont, } \\
\text { Mont. }\end{array}$ & $2 / 25 / 2005$ & E & -15.8 & 7.8 & 5.04 & 9.1 & 3.5 & $<1.7$ & $<1.0$ & 2.6 & 3.0 & 1.8 & 6.9 & 9.7 & 2.1 & 10 & 31 & na & 9.0 & -0.1 \\
\hline $\begin{array}{l}\text { Ned Wilson, } \\
\text { Colo. }\end{array}$ & $4 / 22 / 2005$ & E & -5.6 & 3.9 & 5.47 & 3.4 & 7.0 & $<1.7$ & 1.7 & 0.5 & 3.7 & $<1.0$ & 4.5 & 7.0 & $<0.4$ & 98 & 244 & na & 1.3 & 17.1 \\
\hline $\begin{array}{l}\text { Niwot Snotel, } \\
\text { Colo. }\end{array}$ & $3 / 18 / 2005$ & E & -3.4 & 5.6 & 5.38 & 4.2 & 7.5 & $<1.7$ & 2.4 & 0.5 & 10.6 & 1.2 & 8.8 & 13.5 & $<0.4$ & 30 & 110 & na & 0.7 & 3.2 \\
\hline $\begin{array}{l}\text { Noisy Basin, } \\
\text { Mont. }\end{array}$ & $3 / 4 / 2005$ & E & -9.6 & 4.6 & 5.24 & 5.8 & $<3.1$ & $<1.7$ & $<1.0$ & $<0.4$ & 3.6 & 2.4 & 4.1 & 5.0 & $<0.4$ & 49 & 160 & na & 1.7 & -10.0 \\
\hline Old Battle, Wyo. & $3 / 30 / 2005$ & E & -4.3 & 4.6 & 5.25 & 5.6 & 4.5 & $<1.7$ & 2.2 & 0.5 & 4.0 & $<1.0$ & 5.7 & 7.1 & $<0.4$ & 84 & 250 & na & 2.3 & 13.6 \\
\hline $\begin{array}{l}\text { Old Faithful Fire } \\
\text { Road, Wyo. }\end{array}$ & $2 / 23 / 2005$ & E & -16.2 & 4.3 & 5.27 & 5.4 & $<3.1$ & $<1.7$ & $<1.0$ & $<0.4$ & 9.7 & $<1.0$ & 3.8 & 9.4 & $<0.4$ & 25 & 90 & na & 1.2 & 6.5 \\
\hline $\begin{array}{l}\text { Old Faithful (in } \\
\text { road), Wyo. }\end{array}$ & $2 / 23 / 2005$ & E & 9.6 & 6.1 & 5.86 & 1.4 & 16.5 & 4.9 & 3.4 & 4.3 & 14.9 & 4.1 & 6.9 & 9.5 & 2.0 & na & 41 & na & 6.6 & 20.4 \\
\hline $\begin{array}{l}\text { Phantom Valley, } \\
\text { Colo. }\end{array}$ & $3 / 17 / 2005$ & E & -10.0 & 5.3 & 5.23 & 5.9 & 4.5 & $<1.7$ & 1.4 & 0.6 & 4.5 & $<1.0$ & 4.5 & 8.0 & $<0.4$ & 26 & 92 & na & 1.0 & 14.9 \\
\hline $\begin{array}{l}\text { Rabbit Ears 1, } \\
\text { Colo. }\end{array}$ & $3 / 24 / 2005$ & E & -9.7 & 5.5 & 5.18 & 6.6 & 4.5 & $<1.7$ & 1.2 & 0.4 & 4.7 & $<1.0$ & 7.2 & 8.7 & $<0.4$ & 68 & 243 & 6.1 & 1.7 & 4.9 \\
\hline $\begin{array}{l}\text { Rabbit Ears 2, } \\
\text { Colo. }\end{array}$ & $3 / 24 / 2005$ & E & -10.0 & 5.9 & 5.12 & 7.6 & 4.0 & $<1.7$ & 1.2 & 0.5 & 4.2 & $<1.0$ & 7.2 & 8.9 & $<0.4$ & 66 & 240 & na & 1.7 & 4.1 \\
\hline $\begin{array}{l}\text { Red Mountain, } \\
\text { Mont. }\end{array}$ & $3 / 2 / 2005$ & E & -3.2 & 5.1 & 5.34 & 4.6 & 8.5 & $<1.7$ & $<1.0$ & 1.2 & 5.8 & 1.1 & 4.6 & 7.9 & 0.7 & 16 & 68 & na & 1.6 & 19.0 \\
\hline $\begin{array}{l}\text { Red Mountain } \\
\text { Pass, Colo. }\end{array}$ & $3 / 22 / 2005$ & E & -8.1 & 4.2 & 5.30 & 5.0 & 3.5 & $<1.7$ & 1.1 & 0.5 & 2.9 & $<1.0$ & 3.7 & 5.5 & $<0.4$ & 74 & 225 & na & 2.4 & 17.1 \\
\hline $\begin{array}{l}\text { Rendezvous } \\
\text { Mountain, } \\
\text { Wyo. }\end{array}$ & $3 / 16 / 2005$ & E & -10.4 & 4.7 & 5.61 & 2.5 & 4.0 & $<1.7$ & $<1.0$ & $<0.4$ & 5.1 & $<1.0$ & 3.9 & 4.8 & $<0.4$ & 56 & 197 & na & 1.0 & 14.3 \\
\hline $\begin{array}{l}\text { Ripple Creek } \\
\text { NADP, Colo. }\end{array}$ & $3 / 28 / 2005$ & E & -1.8 & 4.7 & 5.64 & 2.3 & 14.0 & $<1.7$ & 2.9 & 0.7 & 4.5 & 1.2 & 6.3 & 9.4 & $<0.4$ & 48 & 145 & 6.4 & 2.5 & 17.8 \\
\hline
\end{tabular}


[E, depth-integrated environmental; ANC, acid neutralization capacity; $\mu \mathrm{eq} / \mathrm{L}$, microequivalents per liter; $\mu \mathrm{S} / \mathrm{cm}$, microsiemens per centimeter; $\mathrm{SC}$, specific conductance at $25^{\circ} \mathrm{C}$; $\mathrm{pH}$ values in standard units; $\mathrm{H}$, hydrogen; $\mathrm{Ca}$, calcium; $\mathrm{Mg}$, magnesium; $\mathrm{Na}$, sodium; $\mathrm{K}$, potassium; $\mathrm{NH}_{4}$, ammonium; $\mathrm{Cl}$, chloride; $\mathrm{SO}_{4}$, sulfate; $\mathrm{NO}_{3}$, nitrate; DOC, dissolved organic carbon; mg/L, milligrams per liter; $\mathrm{SWE}$, snow/water equivalent; cm, centimeters; NADP, National Atmospheric Deposition Program; $\delta^{34} \mathrm{~S}$, stable sulfur isotope ratio $\left({ }^{34} \mathrm{~S} /{ }^{32} \mathrm{~S}\right)$; Hg, mercury; ng/L, nanograms per liter; na, not analyzed; <, below reporting limits; $\%$, percent]

\begin{tabular}{|c|c|c|c|c|c|c|c|c|c|c|c|c|c|c|c|c|c|c|c|c|}
\hline Site name & $\begin{array}{c}\text { Sample } \\
\text { date }\end{array}$ & $\begin{array}{c}\text { Sample } \\
\text { type }\end{array}$ & $\begin{array}{c}\text { ANC } \\
\text { ( } \mu \text { eq/L) }\end{array}$ & $\underset{(\mu \mathrm{SC} / \mathrm{cm})}{\mathrm{SC}}$ & $\mathrm{pH}$ & $\begin{array}{c}H \\
(\mu \mathrm{eq} / \mathrm{L})\end{array}$ & $\begin{array}{c}\text { Ca } \\
\text { ( } \mu \mathrm{eq} / \mathrm{L})\end{array}$ & $\begin{array}{c}\mathrm{Mg} \\
(\mu \mathrm{eq} / \mathrm{L})\end{array}$ & $\begin{array}{c}\mathrm{Na} \\
(\mu \mathrm{eq} / \mathrm{L})\end{array}$ & $\begin{array}{c}K \\
\text { (peq/L) }\end{array}$ & $\begin{array}{c}\mathbf{N H}_{4} \\
(\mu \mathrm{eq} / \mathrm{L})\end{array}$ & $\underset{(\mu e q / L)}{C l}$ & $\begin{array}{c}\mathrm{SO}_{4} \\
(\mu \mathrm{eq} / \mathrm{L})\end{array}$ & $\begin{array}{c}\mathrm{NO}_{3} \\
(\mu \mathrm{eq} / \mathrm{L})\end{array}$ & $\begin{array}{c}\text { DOC } \\
\text { (mg/L) }\end{array}$ & $\begin{array}{l}\text { SWE } \\
\text { (cm) }\end{array}$ & $\begin{array}{l}\text { Snow } \\
\text { depth } \\
\text { (cm) }\end{array}$ & $\begin{array}{c}\delta^{34} \mathbf{S} \\
\text { (per mil) }\end{array}$ & $\begin{array}{c}\mathrm{Hg} \\
(\mathbf{n g} / \mathrm{L})\end{array}$ & $\begin{array}{c}\text { Ionic } \\
\text { balance } \\
(\%)\end{array}$ \\
\hline $\begin{array}{l}\text { Ripple Creek } \\
\text { Pass, Colo. }\end{array}$ & $3 / 28 / 2005$ & $\mathrm{E}$ & -3.7 & 4.7 & 5.41 & 3.9 & 10.5 & $<1.7$ & 2.9 & 0.6 & 4.9 & 1.1 & 6.5 & 9.2 & $<0.4$ & 44 & 151 & 5.9 & 0.8 & 15.1 \\
\hline $\begin{array}{l}\text { Slumgullion } \\
\text { Pass, Colo. }\end{array}$ & $3 / 23 / 2005$ & E & -6.5 & 4.0 & 5.36 & 4.4 & 5.5 & $<1.7$ & 1.3 & 1.1 & 2.4 & $<1.0$ & 3.6 & 5.1 & 0.5 & 38 & 140 & na & 3.6 & 25.8 \\
\hline $\begin{array}{c}\text { Snow Bowl, } \\
\text { Mont. }\end{array}$ & $3 / 6 / 2005$ & E & -10.9 & 5.1 & 5.12 & 7.6 & 5.0 & $<1.7$ & 1.6 & 1.3 & 3.5 & 1.6 & 4.1 & 4.7 & 0.5 & 37 & 117 & na & 7.6 & 29.0 \\
\hline $\begin{array}{l}\text { South Pass, } \\
\text { Wyo. }\end{array}$ & $3 / 18 / 2005$ & $\mathrm{E}$ & -11.5 & 6.7 & 5.13 & 7.4 & 5.0 & $<1.7$ & $<1.0$ & 1.6 & 5.4 & $<1.0$ & 6.5 & 8.1 & 0.7 & 32 & 125 & 6.3 & 1.7 & 14.1 \\
\hline $\begin{array}{l}\text { Spring Gulch, } \\
\text { Mont. }\end{array}$ & $2 / 25 / 2005$ & E & -3.0 & 4.9 & 5.34 & 4.6 & 8.0 & 2.5 & $<1.0$ & 3.3 & 5.6 & $<1.0$ & 4.4 & 6.6 & 1.1 & 11 & 39 & na & 8.4 & 36.8 \\
\hline $\begin{array}{l}\text { Sunlight Peak, } \\
\text { Colo. }\end{array}$ & $3 / 25 / 2005$ & $\mathrm{E}$ & -4.3 & 4.1 & 5.19 & 6.5 & 3.5 & $<1.7$ & $<1.0$ & $<0.4$ & 3.6 & $<1.0$ & 3.7 & 6.2 & $<0.4$ & 55 & 193 & 4.7 & 0.6 & 15.8 \\
\hline $\begin{array}{l}\text { Sylvan Lake, } \\
\text { Wyo. }\end{array}$ & $2 / 22 / 2005$ & E & -3.5 & 3.9 & 5.35 & 4.5 & $<3.1$ & $<1.7$ & $<1.0$ & $<0.4$ & 6.2 & $<1.0$ & 3.4 & 5.1 & $<0.4$ & 40 & 144 & na & 0.7 & 11.0 \\
\hline $\begin{array}{l}\text { Sylvan Lake (in } \\
\text { road), Wyo. }\end{array}$ & $2 / 22 / 2005$ & E & -7.0 & 4.1 & 5.24 & 5.8 & $<3.1$ & $<1.7$ & $<1.0$ & $<0.4$ & 7.1 & $<1.0$ & 3.6 & 6.2 & $<0.4$ & 35 & 97 & na & 0.9 & 13.4 \\
\hline $\begin{array}{l}\text { Taos Ski Valley, } \\
\text { N. Mex. }\end{array}$ & $3 / 20 / 2005$ & E & -13.9 & 6.0 & 5.19 & 6.5 & 15.0 & $<1.7$ & 4.2 & 1.6 & 5.7 & $<1.0$ & 12.2 & 9.4 & 0.6 & 62 & 201 & na & 10.9 & 20.7 \\
\hline Teton Pass, Wyo. & $3 / 17 / 2005$ & $\mathrm{E}$ & 5.5 & 4.7 & 5.91 & 1.2 & 8.5 & 2.5 & 3.9 & 0.6 & 8.2 & 1.3 & 7.7 & 7.9 & 0.5 & 54 & 188 & na & 2.1 & 5.1 \\
\hline $\begin{array}{l}\text { Togwotee Pass, } \\
\text { Wyo. }\end{array}$ & $3 / 14 / 2005$ & $\mathrm{E}$ & -5.4 & 3.9 & 5.37 & 4.3 & $<3.1$ & $<1.7$ & $<1.0$ & 0.8 & 3.9 & $<1.0$ & 3.1 & 5.3 & $<0.4$ & 27 & 108 & na & 3.0 & 3.0 \\
\hline $\begin{array}{l}\text { Tower Falls, } \\
\text { Wyo. }\end{array}$ & $3 / 1 / 2005$ & E & 0.4 & 4.0 & 5.66 & 2.2 & $<3.1$ & $<1.7$ & $<1.0$ & $<0.4$ & 11.5 & $<1.0$ & 2.8 & 6.6 & $<0.4$ & 8 & 37 & na & 0.4 & 16.5 \\
\hline $\begin{array}{l}\text { Twenty-one } \\
\text { Mile, Mont. }\end{array}$ & $2 / 28 / 2005$ & $\mathrm{E}$ & -4.8 & 4.9 & 5.30 & 5.0 & $<3.1$ & $<1.7$ & $<1.0$ & 1.1 & 6.7 & 1.0 & 3.0 & 6.6 & 0.4 & 23 & 85 & na & 3.7 & 9.3 \\
\hline $\begin{array}{l}\text { University } \\
\text { Camp, Colo. }\end{array}$ & $4 / 14 / 2005$ & $\mathrm{E}$ & -6.3 & 6.0 & 5.18 & 6.6 & 8.0 & $<1.7$ & 1.5 & 1.4 & 7.4 & $<1.0$ & 8.0 & 11.1 & 0.4 & 45 & 147 & na & 3.4 & 13.1 \\
\hline $\begin{array}{l}\text { West Yellow- } \\
\text { stone, Mont. }\end{array}$ & $2 / 24 / 2005$ & E & -6.5 & 4.6 & 5.38 & 4.2 & $<3.1$ & $<1.7$ & $<1.0$ & 0.5 & 8.9 & $<1.0$ & 3.8 & 8.6 & $<0.4$ & 16 & 60 & na & 1.7 & 4.5 \\
\hline $\begin{array}{l}\text { West Yellow- } \\
\text { stone (in } \\
\text { road), Mont. }\end{array}$ & $2 / 24 / 2005$ & $\mathrm{E}$ & 0.1 & 5.2 & 5.75 & 1.8 & 7.0 & $<1.7$ & 1.9 & 0.9 & 15.0 & 1.9 & 6.4 & 9.1 & 0.8 & na & 54 & na & 2.7 & 20.6 \\
\hline $\begin{array}{l}\text { Wolf Creek Pass, } \\
\text { Colo. }\end{array}$ & $3 / 23 / 2005$ & E & -5.4 & 5.0 & 5.22 & 6.0 & 4.0 & $<1.7$ & 1.0 & 0.5 & 3.2 & 1.1 & 5.8 & 7.4 & $<0.4$ & 110 & 323 & na & 3.8 & 1.2 \\
\hline
\end{tabular}


Table 15. Selected chemical and physical data for Rocky Mountain snowpack samples for 2006.

[E, depth-integrated environmental; ANC, acid neutralization capacity; $\mu$ eq/L, microequivalents per liter; $\mu \mathrm{S} / \mathrm{cm}$, microsiemens per centimeter; $\mathrm{SC}$, specific conductance at $25^{\circ} \mathrm{C} ; \mathrm{pH}$ values in standard units; $\mathrm{H}$, hydrogen; $\mathrm{Ca}$, calcium; $\mathrm{Mg}$, magnesium; $\mathrm{Na}$, sodium; $\mathrm{K}$, potassium; $\mathrm{NH}_{4}$, ammonium; $\mathrm{Cl}$, chloride; $\mathrm{SO}_{4}$, sulfate; $\mathrm{NO}_{3}$, nitrate; DOC, dissolved organic carbon; mg/L, milligrams per liter; $\mathrm{SWE}$, snow/water equivalent; cm, centimeters; NADP, National Atmospheric Deposition Program; $\delta^{34} \mathrm{~S}$, stable sulfur isotope ratio $\left({ }^{34} \mathrm{~S} /{ }^{32} \mathrm{~S}\right)$; Hg, mercury; ng/L, nanograms per liter; na, not analyzed; <, below reporting limits;

$\%$, percent]

\begin{tabular}{|c|c|c|c|c|c|c|c|c|c|c|c|c|c|c|c|c|c|c|c|c|}
\hline Site name & $\begin{array}{l}\text { Sample } \\
\text { date }\end{array}$ & $\begin{array}{l}\text { Sample } \\
\text { type }\end{array}$ & $\begin{array}{c}\text { ANC } \\
(\mu \mathrm{eq} / \mathrm{L})\end{array}$ & $\begin{array}{c}\text { SC } \\
(\mu \mathrm{S} / \mathrm{cm})\end{array}$ & $\mathrm{pH}$ & $\begin{array}{c}H \\
(\mu e q / L)\end{array}$ & 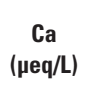 & $\underset{(\mu \mathrm{eq} / \mathrm{L})}{\mathrm{Mg}}$ & $\underset{(\mu \mathrm{eq} / \mathrm{L})}{\mathrm{Na}}$ & $\begin{array}{c}K \\
(\mu e q / L)\end{array}$ & $\begin{array}{c}\mathrm{NH}_{4} \\
(\mu \mathrm{eq} / \mathrm{L})\end{array}$ & $\begin{array}{c}C l \\
(\mu \mathrm{eq} / \mathrm{L})\end{array}$ & $\begin{array}{c}\mathrm{SO}_{4} \\
(\mu \mathrm{eq} / \mathrm{L})\end{array}$ & $\begin{array}{c}\mathrm{NO}_{3} \\
(\mu \mathrm{eq} / \mathrm{L})\end{array}$ & $\begin{array}{c}\text { DOC } \\
\text { (mg/L) }\end{array}$ & $\begin{array}{l}\text { SWE } \\
\text { (cm) }\end{array}$ & $\begin{array}{l}\text { Snow } \\
\text { depth } \\
(\mathrm{cm})\end{array}$ & $\begin{array}{c}\delta^{34} \mathbf{S} \\
\text { (per mil) }\end{array}$ & $\underset{\text { (ng/L) }}{\mathrm{Hg}}$ & $\begin{array}{c}\text { lonic } \\
\text { balance } \\
(\%)\end{array}$ \\
\hline $\begin{array}{l}\text { Apgar Lookout, } \\
\text { Mont. }\end{array}$ & $3 / 10 / 2006$ & E & -4.4 & 4.1 & 5.35 & 4.5 & $<3.1$ & $<1.7$ & 1.5 & $<0.4$ & 5.3 & 1.8 & 3.5 & 5.8 & 0.4 & 64 & 184 & na & 0.7 & 0.8 \\
\hline Banner Summit, Id. & 3/9/2006 & E & -3.9 & 2.8 & 5.46 & 3.5 & $<3.1$ & $<1.7$ & $<1.0$ & $<0.4$ & 2.0 & $<1.0$ & 1.4 & 2.3 & $<0.4$ & 76 & 240 & 4.1 & 0.9 & 18.8 \\
\hline $\begin{array}{l}\text { Berthoud Pass, } \\
\text { Colo. }\end{array}$ & 4/3/2006 & E & 36.2 & 7.0 & 6.54 & 0.3 & 36.9 & 2.5 & 3.3 & 0.8 & 3.2 & 2.8 & 5.5 & 8.6 & na & 70 & 201 & na & 3.7 & -6.2 \\
\hline $\begin{array}{l}\text { Big Mountain, } \\
\text { Mont. }\end{array}$ & $3 / 3 / 2006$ & E & -4.3 & 3.7 & 5.32 & 4.8 & $<3.1$ & $<1.7$ & $<1.0$ & $<0.4$ & 2.8 & 1.3 & 2.3 & 3.8 & $<0.4$ & 98 & 304 & 4.1 & 0.7 & 1.5 \\
\hline Big Sky, Mont. & $3 / 8 / 2006$ & E & 4.0 & 4.1 & 5.88 & 1.3 & 7.0 & $<1.7$ & 3.5 & 0.7 & 5.7 & 3.3 & 5.1 & 4.6 & 0.5 & 41 & 150 & na & 1.6 & 3.6 \\
\hline $\begin{array}{l}\text { Brooklyn Lake, } \\
\text { Wyo. }\end{array}$ & $3 / 30 / 2006$ & E & -4.4 & 5.3 & 5.39 & 4.1 & 7.5 & $<1.7$ & 2.3 & 1.6 & 5.0 & 1.5 & 7.9 & 9.9 & 0.9 & 79 & 230 & 5.3 & 4.8 & 2.8 \\
\hline Brumley, Colo. & $3 / 24 / 2006$ & E & 5.8 & 3.8 & 5.85 & 1.4 & 9.0 & $<1.7$ & 1.2 & $<0.4$ & 3.0 & $<1.0$ & 4.4 & 8.2 & $<0.4$ & 43 & 150 & na & 2.0 & -11.7 \\
\hline Buffalo Pass, Colo. & $3 / 28 / 2006$ & E & -8.7 & 6.2 & 5.09 & 8.1 & 5.0 & $<1.7$ & 1.4 & 0.6 & 5.0 & 1.1 & 7.7 & 11.5 & 0.5 & 122 & 358 & 5.1 & 2.9 & -0.6 \\
\hline $\begin{array}{l}\text { Cameron Pass, } \\
\text { Colo. }\end{array}$ & $3 / 31 / 2006$ & E & -6.5 & 7.0 & 5.08 & 8.3 & 5.0 & $<1.7$ & 3.5 & 0.5 & 4.4 & 3.7 & 6.2 & 9.0 & 0.4 & 68 & 201 & na & 2.4 & 6.7 \\
\hline Canyon, Wyo. & $2 / 22 / 2006$ & E & -3.5 & 3.2 & 5.38 & 4.2 & $<3.1$ & $<1.7$ & $<1.0$ & $<0.4$ & 4.7 & $<1.0$ & 2.0 & 5.2 & $<0.4$ & 28 & 113 & na & 0.9 & 10.4 \\
\hline $\begin{array}{l}\text { Chief Joseph Pass, } \\
\text { Mont. }\end{array}$ & $3 / 6 / 2006$ & E & 0.8 & 3.1 & 5.53 & 3.0 & $<3.1$ & $<1.7$ & 2.0 & $<0.4$ & 2.8 & 2.5 & 2.2 & 3.0 & 0.4 & 55 & 185 & 5.1 & 2.0 & -4.7 \\
\hline Daisy Pass, Mont. & $2 / 28 / 2006$ & E & -2.1 & 2.8 & 5.45 & 3.5 & $<3.1$ & $<1.7$ & $<1.0$ & $<0.4$ & 4.5 & $<1.0$ & 2.3 & 3.7 & $<0.4$ & 77 & 278 & na & 0.7 & 14.4 \\
\hline $\begin{array}{l}\text { Deadman Pass, } \\
\text { Colo. }\end{array}$ & $3 / 31 / 2006$ & $\mathrm{E}$ & 0.3 & 4.8 & 5.42 & 3.8 & 8.5 & $<1.7$ & 1.7 & 0.9 & 4.4 & 1.2 & 6.3 & 10.4 & 0.5 & 56 & 177 & na & 3.2 & 3.0 \\
\hline Divide Peak, Wyo. & $3 / 28 / 2006$ & E & -1.3 & 8.0 & 5.15 & 7.1 & 16.5 & 3.3 & 4.6 & 1.3 & 7.2 & 2.1 & 9.4 & 18.3 & na & 35 & 101 & na & 3.4 & 14.5 \\
\hline Dry Lake, Colo. & $3 / 17 / 2006$ & E & -5.8 & 6.2 & 5.13 & 7.4 & 7.5 & $<1.7$ & 1.8 & $<0.4$ & 4.2 & 1.5 & 6.6 & 16.2 & $<0.4$ & 58 & 185 & 5.7 & 1.2 & -7.4 \\
\hline $\begin{array}{l}\text { Dunckley Pass, } \\
\text { Colo. }\end{array}$ & $3 / 29 / 2006$ & E & 8.4 & 4.5 & 5.99 & 1.0 & 13.0 & 2.5 & 1.8 & 1.4 & 4.9 & 1.5 & 6.1 & 9.9 & $<0.4$ & 65 & 200 & na & 1.7 & -2.6 \\
\hline Elk River, Colo. & $3 / 17 / 2006$ & E & 1.0 & 5.0 & 5.73 & 1.9 & 13.5 & $<1.7$ & 2.5 & 0.7 & 4.2 & 1.6 & 6.4 & 14.3 & 0.4 & 42 & 138 & na & 2.1 & -1.2 \\
\hline Elkhart Park, Wyo. & $3 / 14 / 2006$ & $\mathrm{E}$ & -3.7 & 3.8 & 5.34 & 4.6 & $<3.1$ & $<1.7$ & $<1.0$ & $<0.4$ & 3.2 & 1.0 & 3.5 & 5.8 & $<0.4$ & 31 & 120 & 5.8 & 0.6 & -13.6 \\
\hline $\begin{array}{l}\text { Four Mile Meadow, } \\
\text { Wyo. }\end{array}$ & $3 / 11 / 2006$ & E & -1.0 & 3.0 & 5.62 & 2.4 & $<3.1$ & $<1.7$ & 1.0 & $<0.4$ & 3.6 & 1.4 & 2.1 & 5.2 & 0.4 & 29 & 104 & na & 0.7 & -10.4 \\
\hline $\begin{array}{l}\text { Fremont. Pass, } \\
\text { Colo. }\end{array}$ & 4/6/2006 & E & 17.3 & 5.0 & 6.34 & 0.5 & 17.0 & $<1.7$ & 1.4 & 0.5 & 2.9 & 1.2 & 4.5 & 8.3 & $<0.4$ & 50 & 173 & na & 1.9 & -17.0 \\
\hline Galena Summit, Id. & 3/9/2006 & E & -1.5 & 2.8 & 5.54 & 2.9 & $<3.1$ & $<1.7$ & 1.1 & $<0.4$ & 2.7 & $<1.0$ & 1.8 & 3.0 & 0.4 & 61 & 236 & 5.1 & 2.8 & 16.2 \\
\hline $\begin{array}{l}\text { Garnet Canyon, } \\
\text { Wyo. }\end{array}$ & $3 / 13 / 2006$ & E & -1.3 & 4.0 & 5.66 & 2.2 & 6.5 & $<1.7$ & 2.1 & 0.8 & 6.2 & 1.9 & 4.6 & 6.6 & 0.6 & 63 & 191 & na & 3.1 & 15.5 \\
\hline Grand Mesa, Colo. & $3 / 28 / 2006$ & E & 5.4 & 5.2 & 5.85 & 1.4 & 18.0 & 3.3 & 2.4 & 1.2 & 6.3 & 1.6 & 7.8 & 11.6 & 0.7 & 40 & 136 & na & 4.2 & 10.3 \\
\hline
\end{tabular}


[E, depth-integrated environmental; ANC, acid neutralization capacity; $\mu \mathrm{eq} / \mathrm{L}$, microequivalents per liter; $\mu \mathrm{S} / \mathrm{cm}$, microsiemens per centimeter; $\mathrm{SC}$, specific conductance at $25^{\circ} \mathrm{C}$; $\mathrm{pH}$ values in standard units; $\mathrm{H}$, hydrogen; $\mathrm{Ca}$, calcium; $\mathrm{Mg}$, magnesium; $\mathrm{Na}$, sodium; $\mathrm{K}$, potassium; $\mathrm{NH}_{4}$, ammonium; $\mathrm{Cl}$, chloride; $\mathrm{SO}_{4}$, sulfate; $\mathrm{NO}_{3}$, nitrate; DOC, dissolved organic carbon; mg/L, milligrams per liter; $\mathrm{SWE}$, snow/water equivalent; cm, centimeters; NADP, National Atmospheric Deposition Program; $\delta^{34} \mathrm{~S}$, stable sulfur isotope ratio $\left({ }^{34} \mathrm{~S} /{ }^{32} \mathrm{~S}\right) ; \mathrm{Hg}$, mercury; ng/L, nanograms per liter; na, not analyzed; <, below reporting limits; $\%$, percent]

\begin{tabular}{|c|c|c|c|c|c|c|c|c|c|c|c|c|c|c|c|c|c|c|c|c|}
\hline Site name & $\begin{array}{l}\text { Sample } \\
\text { date }\end{array}$ & $\begin{array}{l}\text { Sample } \\
\text { type }\end{array}$ & $\begin{array}{c}\text { ANC } \\
\text { ( } \mu \text { eq/L) }\end{array}$ & $\begin{array}{c}S C \\
(\mu \mathrm{S} / \mathrm{cm})\end{array}$ & $\mathrm{pH}$ & $\begin{array}{c}\text { H } \\
(\mu \mathrm{eq} / \mathrm{L})\end{array}$ & 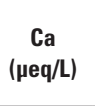 & $\underset{(\mu \mathrm{eq} / \mathrm{L})}{\mathrm{Mg}}$ & $\begin{array}{c}\mathrm{Na} \\
(\mu \mathrm{eq} / \mathrm{L})\end{array}$ & $\begin{array}{c}K \\
(\mu e q / L)\end{array}$ & $\begin{array}{c}\mathrm{NH}_{4} \\
(\mu \mathrm{eq} / \mathrm{L})\end{array}$ & $\underset{(\mu \mathrm{eq} / \mathrm{L})}{\mathrm{Cl}}$ & $\begin{array}{c}\mathrm{SO}_{4} \\
(\mu \mathrm{eq} / \mathrm{L})\end{array}$ & $\begin{array}{c}\mathrm{NO}_{3} \\
(\mu \mathrm{eq} / \mathrm{L})\end{array}$ & $\begin{array}{l}\text { DOC } \\
\text { (mg/L) }\end{array}$ & $\begin{array}{l}\text { SWE } \\
\text { (cm) }\end{array}$ & $\begin{array}{l}\text { Snow } \\
\text { depth } \\
\text { (cm) }\end{array}$ & $\begin{array}{c}\delta^{34} \mathrm{~S} \\
\text { (per mil) }\end{array}$ & $\begin{array}{c}\mathrm{Hg} \\
\text { (ng/L) }\end{array}$ & $\begin{array}{c}\text { Ionic } \\
\text { balance } \\
(\%)\end{array}$ \\
\hline Granite Pass, Mont. & 3/7/2006 & E & 0.2 & 3.6 & 5.37 & 4.3 & $<3.1$ & $<1.7$ & 1.7 & 1.0 & 1.7 & 2.6 & 1.8 & 2.4 & 0.5 & 86 & 257 & na & 5.1 & 10.4 \\
\hline $\begin{array}{l}\text { Gypsum Creek, } \\
\text { Wyo. }\end{array}$ & $3 / 14 / 2006$ & E & -6.7 & 4.3 & 5.29 & 5.1 & $<3.1$ & $<1.7$ & $<1.0$ & 2.2 & 3.5 & 1.3 & 2.9 & 6.3 & 1.2 & 33 & 117 & na & 1.4 & 2.1 \\
\hline Hopewell, N. Mex. & $3 / 25 / 2006$ & E & 31.5 & 9.1 & 6.52 & 0.3 & 45.9 & 5.8 & 4.8 & 3.1 & 8.8 & 3.8 & 11.9 & 19.6 & 1.2 & 23 & 92 & na & 6.2 & 1.4 \\
\hline Kings Hill, Mont. & $2 / 25 / 2006$ & E & -0.8 & 3.9 & 5.47 & 3.4 & $<3.1$ & $<1.7$ & $<1.0$ & 1.1 & 5.1 & 1.3 & 3.4 & 5.8 & 0.8 & 45 & 130 & 6.7 & 9.0 & -4.6 \\
\hline Lake Irene, Colo. & 4/4/2006 & E & 2.2 & 4.1 & 5.60 & 2.5 & 9.5 & $<1.7$ & 1.2 & 1.3 & 4.7 & 1.2 & 6.1 & 10.8 & 0.9 & 65 & 197 & na & 2.3 & -2.8 \\
\hline $\begin{array}{l}\text { Lewis Lake Divide, } \\
\text { Wyo. }\end{array}$ & $2 / 20 / 2006$ & E & -0.4 & 2.7 & 5.44 & 3.6 & $<3.1$ & $<1.7$ & $<1.0$ & $<0.4$ & 4.6 & $<1.0$ & 2.2 & 3.8 & $<0.4$ & 88 & 264 & na & 0.5 & 15.7 \\
\hline Lionshead, Mont. & $2 / 27 / 2006$ & E & 2.8 & 3.7 & 5.67 & 2.1 & $<3.1$ & $<1.7$ & $<1.0$ & 0.7 & 10.0 & 1.2 & 3.8 & 7.4 & 0.4 & 49 & 163 & na & 2.7 & -8.6 \\
\hline $\begin{array}{l}\text { Loch Vale Forest, } \\
\text { Colo. }\end{array}$ & 4/3/2006 & E & 11.2 & 4.9 & 6.01 & 1.0 & 14.5 & $<1.7$ & 1.3 & 0.7 & 4.8 & 1.1 & 6.8 & 10.8 & 0.7 & 90 & 258 & na & 2.7 & -14.8 \\
\hline $\begin{array}{l}\text { Loch Vale Meadow, } \\
\text { Colo. }\end{array}$ & 4/3/2006 & E & 11.3 & 4.8 & 6.26 & 0.5 & 20.5 & 2.5 & 1.3 & $<0.4$ & 3.8 & 1.1 & 5.9 & 10.0 & $<0.4$ & 64 & 173 & na & 1.4 & 0.4 \\
\hline $\begin{array}{l}\text { Loveland Pass, } \\
\text { Colo. }\end{array}$ & 4/7/2006 & E & 105 & 14.7 & 7.19 & 0.1 & 79.3 & 4.9 & 5.9 & 1.1 & 4.3 & 4.5 & 8.4 & 13.8 & na & 70 & 210 & na & 3.5 & $(-15.7)$ \\
\hline Lynx Pass, Colo. & $3 / 18 / 2006$ & E & 5.4 & 3.9 & 5.76 & 1.7 & 7.5 & $<1.7$ & 1.3 & $<0.4$ & 3.1 & 1.0 & 4.4 & 10.2 & $<0.4$ & 49 & 174 & na & 7.0 & -21.5 \\
\hline Medano Pass, Colo. & $3 / 22 / 2006$ & E & 41.3 & 10.6 & 6.04 & 0.9 & 49.4 & 9.1 & 4.2 & 9.3 & 12.0 & 3.1 & 15.9 & 16.6 & 2.8 & 37 & 161 & na & 25.0 & 4.9 \\
\hline Molas Lake, Colo. & $3 / 29 / 2006$ & E & 19.0 & 5.9 & 6.00 & 1.0 & 26.4 & 3.3 & 2.4 & 0.7 & 4.6 & 2.8 & 5.1 & 12.8 & 0.8 & 50 & 177 & 3.9 & 3.1 & -1.6 \\
\hline Monarch Pass, Colo. & $3 / 20 / 2006$ & $\mathrm{E}$ & 37.8 & 7.2 & 6.59 & 0.3 & 38.9 & 4.9 & 2.5 & 1.6 & 5.2 & 2.8 & 6.4 & 8.9 & 0.7 & 38 & 155 & na & 5.5 & -2.3 \\
\hline Monida Pass, Mont. & $2 / 23 / 2006$ & E & 8.5 & 4.4 & 5.86 & 1.4 & 3.5 & $<1.7$ & 1.2 & 1.7 & 14.0 & 1.9 & 4.2 & 7.5 & 1.0 & 19 & 62 & na & 0.6 & -0.7 \\
\hline Mosca Pass, Colo. & $3 / 23 / 2006$ & E & 30.4 & 8.9 & 6.22 & 0.6 & 36.9 & 4.1 & 6.7 & 2.1 & 9.8 & 2.2 & 13.8 & 18.0 & 1.1 & 38 & 147 & na & 3.6 & -3.3 \\
\hline $\begin{array}{l}\text { Mount Belmont, } \\
\text { Mont. }\end{array}$ & $2 / 24 / 2006$ & E & 48.6 & 10.7 & 6.46 & 0.3 & 9.5 & 19.7 & 1.4 & 13.2 & 21.7 & 2.8 & 5.7 & 7.4 & 3.6 & 24 & 77 & na & 33.6 & 1.1 \\
\hline Music Pass, Colo. & $3 / 21 / 2006$ & E & 31.4 & 8.8 & 6.29 & 0.5 & 40.4 & 4.1 & 4.0 & 2.4 & 9.9 & 2.1 & 13.4 & 13.4 & 1.0 & 46 & 180 & 3.5 & 7.9 & 0.9 \\
\hline Niwot Snotel, Colo. & $3 / 3 / 2006$ & $\mathrm{E}$ & 141 & 18.2 & 7.10 & 0.1 & 63.9 & 4.1 & 3.3 & 1.5 & 6.1 & 1.6 & 11.2 & 11.3 & $<0.4$ & 23 & 78 & na & 11.8 & $(-35.3)$ \\
\hline Noisy Basin, Mont. & $3 / 2 / 2006$ & $\mathrm{E}$ & -0.7 & 4.3 & 5.32 & 4.8 & $<3.1$ & $<1.7$ & 1.1 & 1.3 & 3.9 & 1.5 & 2.7 & 4.6 & 0.7 & 102 & 335 & na & 1.2 & 11.1 \\
\hline Old Battle, Wyo. & $3 / 28 / 2006$ & E & -3.9 & 7.0 & 5.16 & 6.9 & 10.0 & 2.5 & 2.3 & 0.7 & 5.6 & 1.7 & 7.8 & 13.2 & 0.5 & 89 & 250 & na & 2.1 & 10.4 \\
\hline $\begin{array}{l}\text { Old Faithful Fire } \\
\text { Road, Wyo. }\end{array}$ & $2 / 22 / 2006$ & E & -3.3 & 3.4 & 5.59 & 2.6 & $<3.1$ & $<1.7$ & 1.1 & $<0.4$ & 6.4 & 1.3 & 2.5 & 6.3 & 0.4 & 26 & 100 & na & 0.9 & 0.5 \\
\hline $\begin{array}{l}\text { Old Faithful (in } \\
\text { road), Wyo. }\end{array}$ & $2 / 22 / 2006$ & $\mathrm{E}$ & 3.4 & 4.0 & 5.84 & 1.4 & 4.5 & $<1.7$ & 2.0 & 1.9 & 9.0 & 3.2 & 3.8 & 6.0 & 1.0 & na & 57 & na & 4.3 & 6.8 \\
\hline $\begin{array}{l}\text { Phantom Valley, } \\
\text { Colo. }\end{array}$ & $3 / 6 / 2006$ & E & -4.2 & 5.7 & 5.24 & 5.8 & 7.5 & $<1.7$ & 1.5 & 0.7 & 4.2 & 1.3 & 7.3 & 12.5 & 0.7 & 20 & 73 & na & 1.0 & -3.7 \\
\hline
\end{tabular}


Table 15. Selected chemical and physical data for Rocky Mountain snowpack samples for 2006.—Continued

[E, depth-integrated environmental; ANC, acid neutralization capacity; $\mu$ eq/L, microequivalents per liter; $\mu \mathrm{S} / \mathrm{cm}$, microsiemens per centimeter; $\mathrm{SC}$, specific conductance at $25^{\circ} \mathrm{C} ; \mathrm{pH}$ values in standard units; $\mathrm{H}$, hydrogen; $\mathrm{Ca}$, calcium; $\mathrm{Mg}$, magnesium; $\mathrm{Na}$, sodium; $\mathrm{K}$, potassium; $\mathrm{NH}_{4}$, ammonium; $\mathrm{Cl}$, chloride; $\mathrm{SO}_{4}$, sulfate; $\mathrm{NO}_{3}$, nitrate; DOC, dissolved organic carbon; mg/L, milligrams per liter; $\mathrm{SWE}$, snow/water equivalent; cm, centimeters; NADP, National Atmospheric Deposition Program; $\delta^{34} \mathrm{~S}$, stable sulfur isotope ratio $\left({ }^{34} \mathrm{~S} /{ }^{32} \mathrm{~S}\right)$; Hg, mercury; ng/L, nanograms per liter; na, not analyzed; <, below reporting limits;

$\%$, percent]

\begin{tabular}{|c|c|c|c|c|c|c|c|c|c|c|c|c|c|c|c|c|c|c|c|c|}
\hline Site name & $\begin{array}{l}\text { Sample } \\
\text { date }\end{array}$ & $\begin{array}{c}\text { Sample } \\
\text { type }\end{array}$ & $\begin{array}{c}\text { ANC } \\
\text { ( } \mu \mathrm{eq} / \mathrm{L})\end{array}$ & $\begin{array}{c}\text { SC } \\
(\mu \mathrm{S} / \mathrm{cm})\end{array}$ & $\mathrm{pH}$ & $\begin{array}{c}H \\
(\mu \mathrm{eq} / \mathrm{L})\end{array}$ & $\begin{array}{c}\text { Ca } \\
(\mu \mathrm{eq} / \mathrm{L})\end{array}$ & $\begin{array}{c}\mathrm{Mg} \\
(\mu \mathrm{eq} / \mathrm{L})\end{array}$ & $\underset{(\mu \mathrm{Na} / \mathrm{L})}{\mathrm{Na}}$ & $\begin{array}{c}K \\
(\mu \mathrm{eq} / \mathrm{L})\end{array}$ & $\begin{array}{c}\mathrm{NH}_{4} \\
(\mu \mathrm{eq} / \mathrm{L})\end{array}$ & $\begin{array}{c}C I \\
(\mu \mathrm{eq} / \mathrm{L})\end{array}$ & $\begin{array}{c}\mathrm{SO}_{4} \\
(\mu \mathrm{eq} / \mathrm{L})\end{array}$ & $\begin{array}{c}\mathrm{NO}_{3} \\
(\mu \mathrm{eq} / \mathrm{L})\end{array}$ & $\begin{array}{c}\text { DOC } \\
(\mathrm{mg} / \mathrm{L})\end{array}$ & $\begin{array}{l}\text { SWE } \\
\text { (cm) }\end{array}$ & $\begin{array}{l}\text { Snow } \\
\text { depth } \\
\text { (cm) }\end{array}$ & $\begin{array}{c}\delta^{34} \mathbf{S} \\
\text { (per mil) }\end{array}$ & $\begin{array}{c}\mathrm{Hg} \\
\text { (ng/L) }\end{array}$ & $\begin{array}{c}\text { lonic } \\
\text { balance } \\
(\%)\end{array}$ \\
\hline Rabbit Ears 1, Colo. & $3 / 27 / 2006$ & E & -4.8 & 5.1 & 5.30 & 5.0 & 7.5 & $<1.7$ & 1.7 & 0.6 & 5.1 & 1.2 & 7.2 & 11.0 & 0.4 & 107 & 282 & na & 1.2 & 1.2 \\
\hline Rabbit Ears 2, Colo. & $3 / 27 / 2006$ & E & -5.1 & 5.5 & 5.34 & 4.6 & 9.0 & $<1.7$ & 2.0 & 0.5 & 5.3 & 1.3 & 7.8 & 12.1 & $<0.4$ & 106 & 292 & na & 1.1 & 0.1 \\
\hline $\begin{array}{l}\text { Red Mountain, } \\
\text { Mont. }\end{array}$ & $3 / 4 / 2006$ & $\mathrm{E}$ & -0.2 & 4.1 & 5.66 & 2.2 & 6.0 & $<1.7$ & 1.8 & 1.2 & 7.4 & 2.1 & 4.0 & 7.2 & 1.1 & 40 & 134 & na & 2.6 & 16.4 \\
\hline $\begin{array}{l}\text { Red Mountain Pass, } \\
\text { Colo. }\end{array}$ & $3 / 29 / 2006$ & $\mathrm{E}$ & 10.5 & 4.9 & 6.03 & 0.9 & 17.5 & 2.5 & 2.2 & 1.5 & 4.5 & 2.2 & 5.5 & 11.6 & 0.4 & 63 & 221 & na & 3.0 & -1.3 \\
\hline $\begin{array}{l}\text { Rendezvous Moun- } \\
\text { tain, Wyo. }\end{array}$ & $3 / 15 / 2006$ & E & 2.0 & 3.0 & 5.80 & 1.6 & $<3.1$ & $<1.7$ & 1.9 & $<0.4$ & 4.7 & 1.6 & 3.5 & 4.1 & 0.4 & 77 & 265 & na & 1.4 & -14.9 \\
\hline $\begin{array}{l}\text { Ripple Creek } \\
\text { NADP, Colo. }\end{array}$ & $3 / 29 / 2006$ & $\mathrm{E}$ & 3.7 & 4.3 & 5.92 & 1.2 & 12.5 & 2.5 & 1.9 & $<0.4$ & 4.2 & 1.3 & 4.7 & 10.1 & na & 64 & 167 & 5.7 & 2.0 & 5.7 \\
\hline $\begin{array}{l}\text { Slumgullion Pass, } \\
\text { Colo. }\end{array}$ & $3 / 30 / 2006$ & $\mathrm{E}$ & 15.0 & 4.2 & 6.31 & 0.5 & 18.5 & $<1.7$ & 1.4 & 1.1 & 2.7 & 1.4 & 3.9 & 7.0 & 1.2 & 35 & 134 & 4.2 & 3.3 & -6.1 \\
\hline Snow Bowl, Mont. & $3 / 5 / 2006$ & $\mathrm{E}$ & -3.0 & 3.3 & 5.42 & 3.8 & $<3.1$ & $<1.7$ & 1.6 & 0.5 & 3.2 & 1.8 & 2.6 & 3.4 & 0.6 & 75 & 233 & na & 2.1 & 7.7 \\
\hline South Pass, Wyo. & $3 / 29 / 2006$ & $\mathrm{E}$ & -3.4 & 6.5 & 5.13 & 7.4 & 7.5 & $<1.7$ & 2.9 & 0.5 & 5.1 & 1.5 & 8.7 & 8.6 & na & 43 & 136 & na & 0.9 & 10.7 \\
\hline Spring Gulch, Mont. & $2 / 24 / 2006$ & E & 3.7 & 4.1 & 5.76 & 1.7 & 9.0 & 2.5 & $<1.0$ & 4.6 & 7.0 & 1.5 & 3.8 & 8.0 & 2.5 & 21 & 68 & na & 4.2 & 18.5 \\
\hline Sunlight Peak, Colo. & $3 / 30 / 2006$ & $\mathrm{E}$ & -0.3 & 4.2 & 5.77 & 1.7 & 10.5 & $<1.7$ & 2.1 & 0.5 & 5.9 & 1.3 & 6.6 & 9.8 & 0.8 & 69 & 224 & na & 2.0 & 7.6 \\
\hline Sylvan Lake, Wyo. & $2 / 21 / 2006$ & $\mathrm{E}$ & -2.4 & 2.9 & 5.49 & 3.2 & $<3.1$ & $<1.7$ & $<1.0$ & $<0.4$ & 4.4 & $<1.0$ & 2.4 & 4.4 & $<0.4$ & 45 & 148 & na & 1.0 & 6.1 \\
\hline $\begin{array}{l}\text { Sylvan Lake (in } \\
\text { road), Wyo. }\end{array}$ & $2 / 21 / 2006$ & E & -4.3 & 3.0 & 5.49 & 3.2 & $<3.1$ & $<1.7$ & $<1.0$ & $<0.4$ & 4.7 & $<1.0$ & 2.6 & 4.9 & 0.4 & 40 & 103 & na & 0.8 & 2.8 \\
\hline $\begin{array}{l}\text { Taos Ski Valley, } \\
\text { N. Mex. }\end{array}$ & $3 / 26 / 2006$ & $\mathrm{E}$ & 37.6 & 12.1 & 6.47 & 0.3 & 61.4 & 6.6 & 6.3 & 3.5 & 9.7 & 4.5 & 26.2 & 21.3 & 1.5 & 20 & 79 & 4.0 & 11.3 & -1.0 \\
\hline Teton Pass, Wyo. & $3 / 16 / 2006$ & $\mathrm{E}$ & 2.4 & 3.7 & 5.76 & 1.7 & 5.0 & $<1.7$ & 2.7 & 1.4 & 5.7 & 2.2 & 4.1 & 5.2 & na & 75 & 268 & na & 2.2 & 8.8 \\
\hline $\begin{array}{l}\text { Togwotee Pass, } \\
\text { Wyo. }\end{array}$ & $3 / 11 / 2006$ & $\mathrm{E}$ & -1.7 & 3.3 & 5.44 & 3.6 & $<3.1$ & $<1.7$ & 1.1 & $<0.4$ & 3.1 & 1.2 & 2.6 & 4.6 & 3.8 & 49 & 156 & na & 1.2 & -2.9 \\
\hline $\begin{array}{l}\text { Twenty-one Mile, } \\
\text { Mont. }\end{array}$ & $2 / 27 / 2006$ & E & -1.1 & 3.5 & 5.54 & 2.9 & $<3.1$ & $<1.7$ & $<1.0$ & 1.1 & 8.0 & 1.4 & 2.9 & 6.5 & 0.8 & 35 & 128 & na & 1.9 & 5.3 \\
\hline $\begin{array}{l}\text { University Camp, } \\
\text { Colo. }\end{array}$ & $4 / 5 / 2006$ & E & 44.8 & 8.6 & 6.61 & 0.2 & 35.4 & 3.3 & 2.1 & 1.2 & 5.6 & 1.3 & 8.8 & 10.5 & 0.5 & 55 & 164 & na & 4.6 & $(-15.4)$ \\
\hline $\begin{array}{l}\text { West Yellowstone, } \\
\text { Mont. }\end{array}$ & $2 / 23 / 2006$ & $\mathrm{E}$ & -1.2 & 3.7 & 5.61 & 2.5 & $<3.1$ & $<1.7$ & $<1.0$ & 0.4 & 8.5 & 1.1 & 2.8 & 7.5 & 0.4 & 27 & 102 & na & 0.4 & 0.0 \\
\hline $\begin{array}{l}\text { West Yellowstone } \\
\text { (in road), Mont. }\end{array}$ & $2 / 23 / 2006$ & $\mathrm{E}$ & 1.6 & 3.9 & 5.72 & 1.9 & $<3.1$ & $<1.7$ & 1.0 & 0.4 & 11.3 & 1.3 & 3.8 & 7.9 & 0.9 & na & 63 & na & $<0.4$ & 0.3 \\
\hline $\begin{array}{l}\text { Wolf Creek Pass, } \\
\text { Colo. }\end{array}$ & $3 / 30 / 2006$ & E & -1.9 & 6.2 & 5.50 & 3.2 & 14.5 & 2.5 & 3.6 & 1.1 & 7.6 & 3.5 & 9.4 & 15.8 & 0.5 & 56 & 210 & na & 5.9 & 6.0 \\
\hline
\end{tabular}


[E, depth-integrated environmental; ANC, acid neutralization capacity; $\mu \mathrm{eq} / \mathrm{L}$, microequivalents per liter; $\mu \mathrm{S} / \mathrm{cm}$, microsiemens per centimeter; $\mathrm{SC}$, specific conductance at $25^{\circ} \mathrm{C}$; $\mathrm{pH}$ values in standard units; $\mathrm{H}$, hydrogen; $\mathrm{Ca}$, calcium; $\mathrm{Mg}$, magnesium; $\mathrm{Na}$, sodium; $\mathrm{K}$, potassium; $\mathrm{NH}_{4}$, ammonium; $\mathrm{Cl}$, chloride; $\mathrm{SO}_{4}$, sulfate; $\mathrm{NO}_{3}$, nitrate; DOC, dissolved organic carbon; mg/L, milligrams per liter; $\mathrm{SWE}$, snow/water equivalent; cm, centimeters; NADP, National Atmospheric Deposition Program; $8^{34} \mathrm{~S}$, stable sulfur isotope ratio $\left({ }^{34} \mathrm{~S} /{ }^{2} \mathrm{~S}\right) ; \mathrm{Hg}$, mercury; ng $/ \mathrm{L}$, nanograms per liter; na, not analyzed; <, below reporting limits; \%, percent]

\begin{tabular}{|c|c|c|c|c|c|c|c|c|c|c|c|c|c|c|c|c|c|c|c|c|}
\hline Site name & $\begin{array}{l}\text { Sample } \\
\text { date }\end{array}$ & $\begin{array}{c}\text { Sample } \\
\text { type }\end{array}$ & $\begin{array}{c}\text { ANC } \\
(\mu e q / L)\end{array}$ & $\begin{array}{c}\text { SC } \\
(\mu \mathrm{S} / \mathrm{cm})\end{array}$ & $\mathrm{pH}$ & $\begin{array}{c}\text { H } \\
(\mu \mathrm{eq} / \mathrm{L})\end{array}$ & $\begin{array}{c}\text { Ca } \\
(\mu \mathrm{eq} / \mathrm{L})\end{array}$ & $\begin{array}{c}M g \\
\text { (peq/L) }\end{array}$ & $\begin{array}{c}\mathrm{Na} \\
(\mu \mathrm{eq} / \mathrm{L})\end{array}$ & $\begin{array}{c}K \\
\text { ( } \mu \text { eq/L) }\end{array}$ & $\begin{array}{c}\mathrm{NH}_{4} \\
(\mu \mathrm{eq} / \mathrm{L})\end{array}$ & $\underset{(\mu e q / L)}{C l}$ & 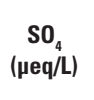 & $\begin{array}{c}\mathrm{NO}_{3} \\
(\mu \mathrm{eq} / \mathrm{L})\end{array}$ & $\begin{array}{c}\text { DOC } \\
\text { (mg/L) }\end{array}$ & $\begin{array}{l}\text { SWE } \\
(\mathrm{cm})\end{array}$ & $\begin{array}{l}\text { Snow } \\
\text { depth } \\
\text { (cm) }\end{array}$ & $\begin{array}{c}\delta^{34} \mathrm{~S} \\
\text { (per mil) }\end{array}$ & $\begin{array}{c}\mathrm{Hg} \\
(\mathbf{n g} / \mathrm{L})\end{array}$ & $\begin{array}{c}\text { lonic } \\
\text { balance } \\
(\%)\end{array}$ \\
\hline $\begin{array}{l}\text { Apgar Lookout, } \\
\text { Mont. }\end{array}$ & $3 / 13 / 2007$ & E & -4.4 & 4.7 & 5.24 & 5.8 & 4.0 & $<1.7$ & 1.4 & 0.6 & 4.6 & 1.5 & 4.0 & 6.4 & $<0.4$ & 59 & 153 & na & 2.0 & 16.0 \\
\hline $\begin{array}{l}\text { Banner Summit, } \\
\text { Idaho }\end{array}$ & $3 / 8 / 2007$ & E & 0.8 & 3.8 & 5.56 & 2.8 & 4.5 & $<1.7$ & 4.4 & 0.6 & 3.1 & 1.6 & 3.8 & 4.1 & $<0.4$ & 50 & 160 & na & 1.7 & 19.1 \\
\hline $\begin{array}{l}\text { Berthoud Pass, } \\
\text { Colo. }\end{array}$ & $3 / 30 / 2007$ & E & -2.9 & 4.9 & 5.27 & 5.4 & 6.2 & $<1.7$ & 2.3 & 1.5 & 1.3 & 2.4 & 3.8 & 8.1 & 0.7 & 46 & 173 & na & 3.0 & 7.7 \\
\hline $\begin{array}{l}\text { Big Mountain, } \\
\text { Mont. }\end{array}$ & $3 / 2 / 2007$ & E & -5.8 & 4.0 & 5.23 & 5.9 & $<3.1$ & $<1.7$ & 1.4 & $<0.4$ & 2.1 & 1.4 & 3.2 & 4.1 & $<0.4$ & 70 & 260 & na & 0.9 & 3.9 \\
\hline Big Sky, Mont. & $3 / 7 / 2007$ & E & 1.1 & 3.2 & 5.56 & 2.8 & 4.6 & $<1.7$ & $<1.0$ & 0.6 & 3.7 & $<1.0$ & 2.7 & 3.8 & $<0.4$ & 32 & 114 & na & 0.8 & 21.0 \\
\hline $\begin{array}{l}\text { Brooklyn Lake, } \\
\text { Wyo. }\end{array}$ & $3 / 27 / 2007$ & E & 5.6 & 4.5 & 5.65 & 2.2 & 12.4 & 3.4 & 1.6 & 5.2 & $<1.0$ & 1.7 & 7.5 & 8.5 & 1.5 & 58 & 153 & na & 3.5 & 2.8 \\
\hline Brumley, Colo. & $3 / 22 / 2007$ & E & 7.4 & 4.9 & 5.60 & 2.5 & 17.8 & 2.0 & 3.6 & 1.7 & 3.8 & 1.3 & 8.4 & 10.5 & 0.6 & 34 & 122 & na & 1.3 & 6.3 \\
\hline Buffalo Pass, Colo. & $4 / 3 / 2007$ & E & -6.0 & 6.0 & 5.18 & 6.6 & 8.6 & 1.9 & 1.2 & 1.5 & 5.2 & 1.1 & 8.7 & 11.5 & 0.6 & 89 & 290 & na & 2.0 & 8.0 \\
\hline Cameron Pass, Colo. & $3 / 28 / 2007$ & E & -1.9 & 6.5 & 5.28 & 5.2 & 8.8 & $<1.7$ & 12.8 & 1.7 & 3.2 & 13.5 & 5.1 & 8.7 & 0.6 & 58 & 179 & na & 3.6 & 7.6 \\
\hline Canyon, Wyo. & $2 / 21 / 2007$ & E & 1.0 & 3.7 & 5.67 & 2.1 & 5.2 & $<1.7$ & 1.5 & $<0.4$ & 7.0 & 1.2 & 4.3 & 6.1 & $<0.4$ & 25 & 124 & na & 2.6 & 11.2 \\
\hline $\begin{array}{l}\text { Chief Joseph Pass, } \\
\text { Mont. }\end{array}$ & $3 / 5 / 2007$ & E & -1.7 & 3.5 & 5.45 & 3.5 & $<3.1$ & $<1.7$ & 2.9 & 0.7 & 3.0 & 1.0 & 2.9 & 3.5 & 0.4 & 39 & 140 & na & 4.2 & 16.2 \\
\hline Daisy Pass, Mont. & $2 / 27 / 2007$ & E & 3.1 & 3.6 & 5.75 & 1.8 & 5.2 & $<1.7$ & 1.4 & 1.8 & 5.0 & 1.3 & 4.5 & 5.1 & 0.4 & 68 & 250 & na & 4.0 & 4.1 \\
\hline Divide Peak, Wyo. & $3 / 27 / 2007$ & E & 13.0 & 7.1 & 5.91 & 1.2 & 25.3 & 5.2 & 2.8 & 1.9 & 7.1 & 1.8 & 10.5 & 16.9 & 0.9 & 44 & 117 & na & 3.4 & 1.6 \\
\hline Dry Lake, Colo. & $3 / 16 / 2007$ & E & -9.0 & 7.0 & 5.07 & 8.5 & 11.9 & 1.8 & 1.1 & 0.6 & 4.9 & 1.3 & 7.6 & 15.9 & $<0.4$ & 36 & 132 & na & 0.7 & 7.6 \\
\hline $\begin{array}{l}\text { Dunckley Pass, } \\
\text { Colo. }\end{array}$ & $3 / 28 / 2007$ & E & 2.6 & 4.8 & 5.70 & 2.0 & 13.4 & 2.3 & 1.3 & 3.2 & 4.3 & 1.2 & 6.1 & 10.4 & 0.9 & 49 & 164 & na & 1.9 & 12.9 \\
\hline Elk River, Colo. & $3 / 16 / 2007$ & $\mathrm{E}$ & 7.5 & 5.7 & 5.86 & 1.4 & 27.6 & 2.9 & 1.6 & 4.5 & 3.0 & 1.6 & 7.3 & 14.3 & 0.7 & 35 & 117 & na & 0.5 & 14.2 \\
\hline Elkhart Park, Wyo. & $3 / 14 / 2007$ & E & -5.3 & 4.4 & 5.33 & 4.7 & 16.6 & $<1.7$ & 1.2 & 0.6 & 3.6 & $<1.0$ & 4.1 & 6.7 & 0.5 & 23 & 87 & na & 1.1 & 42.7 \\
\hline $\begin{array}{l}\text { Four Mile Meadow, } \\
\text { Wyo. }\end{array}$ & $3 / 12 / 2007$ & E & -0.2 & 3.7 & 5.53 & 3.0 & 4.7 & $<1.7$ & 1.4 & 0.6 & 5.0 & 1.1 & 3.5 & 5.9 & 0.5 & 23 & 88 & na & 2.4 & 16.3 \\
\hline Fremont Pass, Colo. & $4 / 6 / 2007$ & E & 0.5 & 4.6 & 5.55 & 2.8 & 9.5 & 1.9 & 1.9 & 2.5 & 2.0 & 1.3 & 5.3 & 8.5 & 0.8 & 36 & 146 & na & 3.7 & 14.0 \\
\hline $\begin{array}{l}\text { Galena Summit, } \\
\text { Idaho }\end{array}$ & $3 / 8 / 2007$ & E & 3.5 & 3.7 & 5.66 & 2.2 & 5.8 & $<1.7$ & 2.3 & 1.8 & 2.3 & 1.6 & 3.2 & 5.0 & 0.5 & 35 & 146 & na & 2.5 & 3.9 \\
\hline $\begin{array}{l}\text { Garnet Canyon, } \\
\text { Wyo. }\end{array}$ & $3 / 23 / 2007$ & E & 0.7 & 4.4 & 5.50 & 3.2 & 6.0 & $<1.7$ & 1.4 & 1.9 & 5.0 & 1.3 & 4.7 & 6.4 & 0.8 & 38 & 110 & na & 4.9 & 14.3 \\
\hline Grand Mesa, Colo. & $3 / 26 / 2007$ & E & 17.7 & 6.3 & 6.04 & 0.9 & 23.0 & 5.7 & 2.9 & 7.1 & 2.6 & 2.4 & 11.6 & 13.8 & 1.8 & 33 & 113 & na & 3.2 & -3.9 \\
\hline Granite Pass, Mont. & $3 / 6 / 2007$ & E & 16.0 & 4.4 & 5.59 & 2.6 & $<3.1$ & $<1.7$ & 1.6 & 1.4 & $<1.0$ & 1.9 & 2.5 & 1.8 & 0.7 & 54 & 172 & na & 7.1 & -59.8 \\
\hline
\end{tabular}


Table 16. Selected chemical and physical data for Rocky Mountain snowpack samples for 2007.—Continued

E, depth-integrated environmental; ANC, acid neutralization capacity; $\mu$ eq/L, microequivalents per liter; $\mu \mathrm{S} / \mathrm{cm}$, microsiemens per centimeter; $\mathrm{SC}$, specific conductance at $25^{\circ} \mathrm{C} ; \mathrm{pH}$ values in standard units; $\mathrm{H}$, hydrogen; Ca, calcium; Mg, magnesium; $\mathrm{Na}$, sodium; $\mathrm{K}$, potassium; $\mathrm{NH}_{4}$, ammonium; $\mathrm{Cl}$, chloride; $\mathrm{SO}_{4}$, sulfate; $\mathrm{NO}_{3}$, nitrate; DOC, dissolved organic carbon; mg/L, milligrams per liter; $\mathrm{SWE}$, snow/water equivalent; cm, centimeters; NADP, National Atmospheric Deposition Program; $\delta^{34} \mathrm{~S}$, stable sulfur isotope ratio $\left({ }^{34} \mathrm{~S} /{ }^{32} \mathrm{~S}\right) ; \mathrm{Hg}$, mercury; ng/L, nanograms per liter; na, not analyzed; <, below reporting limits; \%, percent]

\begin{tabular}{|c|c|c|c|c|c|c|c|c|c|c|c|c|c|c|c|c|c|c|c|c|}
\hline Site name & $\begin{array}{l}\text { Sample } \\
\text { date }\end{array}$ & $\begin{array}{l}\text { Sample } \\
\text { type }\end{array}$ & $\begin{array}{c}\text { ANC } \\
\text { ( } \mu \text { eq/L) }\end{array}$ & $\begin{array}{c}\mathrm{SC} \\
(\mu \mathrm{S} / \mathrm{cm})\end{array}$ & $\mathrm{pH}$ & 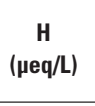 & 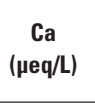 & $\underset{(\mu \mathrm{eq} / \mathrm{L})}{\mathrm{Mg}}$ & $\begin{array}{c}\mathrm{Na} \\
(\mu \mathrm{eq} / \mathrm{L})\end{array}$ & $\begin{array}{c}K \\
(\mu e q / L)\end{array}$ & $\begin{array}{c}\mathrm{NH}_{4} \\
\text { ( } \mu \mathrm{eq} / \mathrm{L})\end{array}$ & 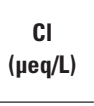 & $\begin{array}{c}\mathrm{SO}_{4} \\
(\mu \mathrm{eq} / \mathrm{L})\end{array}$ & $\begin{array}{c}\mathrm{NO}_{3} \\
(\mu \mathrm{eq} / \mathrm{L})\end{array}$ & $\begin{array}{l}\text { DOC } \\
\text { (mg/L) }\end{array}$ & $\begin{array}{l}\text { SWE } \\
\text { (cm) }\end{array}$ & $\begin{array}{l}\text { Snow } \\
\text { depth } \\
\text { (cm) }\end{array}$ & $\begin{array}{c}\delta^{34} \mathbf{S} \\
\text { (per mil) }\end{array}$ & $\underset{(\mathbf{n g} / \mathrm{L})}{\mathrm{Hg}}$ & $\begin{array}{c}\text { Ionic } \\
\text { balance } \\
(\%) \\
\end{array}$ \\
\hline Grizzly Ridge, Utah & $3 / 30 / 2007$ & E & 13.9 & 5.4 & 6.20 & 0.6 & 19.8 & 4.6 & 2.7 & 1.8 & 5.7 & 1.7 & 6.1 & 11.4 & 0.8 & 24 & 91 & na & 5.1 & 3.2 \\
\hline $\begin{array}{l}\text { Gypsum Creek, } \\
\text { Wyo. }\end{array}$ & $3 / 14 / 2007$ & E & -1.4 & 4.7 & 5.33 & 4.7 & 7.5 & 2.2 & 1.1 & 5.5 & $<1.0$ & 1.6 & 4.7 & 6.9 & 1.7 & 22 & 80 & na & 2.5 & 22.9 \\
\hline Hopewell, N. Mex. & $3 / 24 / 2007$ & E & 4.2 & 5.7 & 5.77 & 1.7 & 16.5 & 2.6 & 2.3 & 1.8 & 7.6 & 2.4 & 9.1 & 12.7 & 0.5 & 27 & 98 & na & 6.6 & 6.7 \\
\hline Kings Hill, Mont. & $2 / 24 / 2007$ & E & 15.9 & 5.5 & 6.12 & 0.8 & 6.8 & 1.9 & 2.0 & 2.5 & 14.0 & 2.4 & 6.0 & 8.2 & 1.0 & 33 & 121 & na & 13.5 & -7.5 \\
\hline Lake Fork, Utah & $3 / 30 / 2007$ & E & 16.4 & 5.7 & 6.17 & 0.7 & 18.3 & 4.3 & 2.4 & 2.4 & 8.0 & 2.0 & 6.7 & 10.7 & 0.8 & 28 & 111 & na & 5.7 & 0.3 \\
\hline Lake Irene, Colo. & $4 / 5 / 2007$ & E & 2.5 & 4.5 & 5.47 & 3.4 & 6.9 & $<1.7$ & $<1.0$ & 9.2 & $<1.0$ & 1.8 & 5.0 & 8.8 & 1.6 & 58 & 186 & na & 4.8 & 3.6 \\
\hline $\begin{array}{l}\text { Lewis Lake Divide, } \\
\text { Wyo. }\end{array}$ & 2/19/2007 & $\mathrm{E}$ & 4.7 & 3.7 & 5.79 & 1.6 & 4.1 & $<1.7$ & 1.3 & $<0.4$ & 8.5 & 1.0 & 4.1 & 5.6 & $<0.4$ & 59 & 203 & na & 1.2 & 0.4 \\
\hline Lionshead, Mont. & 2/26/2007 & E & 6.0 & 5.5 & 5.89 & 1.3 & 11.5 & 2.2 & 2.2 & 1.4 & 13.1 & 2.4 & 6.7 & 10.9 & 0.7 & 36 & 153 & na & 8.9 & 9.9 \\
\hline $\begin{array}{l}\text { Loch Vale Forest, } \\
\text { Colo. }\end{array}$ & $4 / 3 / 2007$ & E & -9.5 & 5.5 & 5.28 & 5.2 & 8.8 & $<1.7$ & 1.2 & 1.5 & 4.7 & 1.3 & 6.3 & 11.4 & 0.8 & 78 & 215 & na & 4.8 & 6.1 \\
\hline $\begin{array}{l}\text { Loch Vale Meadow, } \\
\text { Colo. }\end{array}$ & $4 / 3 / 2007$ & E & -3.7 & 4.8 & 5.30 & 5.0 & 6.7 & $<1.7$ & 1.2 & 0.5 & 4.8 & $<1.0$ & 5.5 & 9.9 & $<0.4$ & 62 & 178 & na & 0.3 & 8.4 \\
\hline Lone Pine, Colo. & $3 / 29 / 2007$ & E & -1.3 & 4.8 & 5.40 & 4.0 & 8.8 & 2.1 & 1.1 & 2.8 & $<1.0$ & 1.2 & 5.2 & 8.2 & 1.4 & 46 & 133 & na & 5.1 & 12.4 \\
\hline $\begin{array}{l}\text { Loveland Pass, } \\
\text { Colo. }\end{array}$ & $4 / 7 / 2007$ & E & -5.2 & 4.9 & 5.28 & 5.2 & 5.6 & $<1.7$ & 1.9 & 0.4 & 2.6 & 2.7 & 3.7 & 8.7 & $<0.4$ & 52 & 189 & na & 0.9 & 2.8 \\
\hline Medano Pass, Colo. & $3 / 21 / 2007$ & E & 11.8 & 6.3 & 5.63 & 2.3 & 20.0 & 5.4 & 2.2 & 11.7 & $<1.0$ & 2.5 & 9.4 & 9.2 & 2.1 & 46 & 155 & na & 25.6 & 11.7 \\
\hline Molas Lake, Colo. & $3 / 27 / 2007$ & E & 28.5 & 7.2 & 6.55 & 0.3 & 41.0 & 3.7 & 2.7 & 0.9 & 3.8 & 2.3 & 7.1 & 12.1 & 0.4 & 42 & 128 & na & 2.7 & 2.3 \\
\hline Monarch Pass, Colo. & $3 / 19 / 2007$ & E & 34.2 & 7.1 & 6.38 & 0.4 & 26.3 & 6.6 & 2.8 & 19.6 & $<1.0$ & 5.2 & 8.9 & 6.9 & 3.6 & 30 & 116 & na & 7.6 & 0.5 \\
\hline $\begin{array}{l}\text { Mount Belmont, } \\
\text { Mont. }\end{array}$ & $2 / 23 / 2007$ & E & 6.2 & 6.5 & 5.53 & 3.0 & 13.9 & 4.8 & 2.4 & 9.0 & $<1.0$ & 3.1 & 7.8 & 6.5 & 3.3 & 11 & 45 & na & 30.3 & 16.6 \\
\hline Music Pass, Colo. & $3 / 20 / 2007$ & E & -2.6 & 5.1 & 5.41 & 3.9 & 14.4 & 2.5 & 2.4 & 3.1 & 2.5 & 1.2 & 8.2 & 9.7 & 1.1 & 87 & 244 & na & 7.0 & 20.3 \\
\hline Noisy Basin, Mont. & $3 / 1 / 2007$ & E & -4.3 & 4.2 & 5.29 & 5.1 & $<3.1$ & $<1.7$ & 1.4 & 0.5 & 3.1 & 1.8 & 4.0 & 4.9 & $<0.4$ & 72 & 285 & na & 1.6 & -2.6 \\
\hline Old Battle, Wyo. & $3 / 26 / 2007$ & $\mathrm{E}$ & 0.0 & 5.4 & 5.51 & 3.1 & 13.5 & 2.6 & 1.7 & 0.7 & 4.2 & 1.3 & 7.7 & 12.2 & 0.4 & 61 & 167 & na & 3.4 & 9.9 \\
\hline $\begin{array}{l}\text { Old Faithful Fire } \\
\text { Road, Wyo. }\end{array}$ & $2 / 21 / 2007$ & E & 4.4 & 3.9 & 5.92 & 1.2 & 5.0 & $<1.7$ & 2.2 & $<0.4$ & 8.3 & 1.3 & 4.6 & 5.8 & 0.4 & 23 & 102 & na & 1.3 & 1.6 \\
\hline $\begin{array}{l}\text { Old Faithful (in } \\
\text { road), Wyo. }\end{array}$ & $2 / 21 / 2007$ & E & 14.4 & 6.0 & 6.18 & 0.7 & 13.4 & 3.4 & 5.4 & 3.7 & 10.4 & 5.4 & 7.0 & 8.0 & 1.1 & na & 49 & na & 14.9 & 2.9 \\
\hline $\begin{array}{l}\text { Phantom Valley, } \\
\text { Colo. }\end{array}$ & $3 / 8 / 2007$ & E & -3.5 & 5.8 & 5.21 & 6.2 & 6.4 & $<1.7$ & $<1.0$ & 1.0 & 2.5 & $<1.0$ & 5.7 & 9.8 & 0.5 & 32 & 116 & na & 2.4 & 2.0 \\
\hline Rabbit Ears 1, Colo. & $3 / 28 / 2007$ & E & -5.5 & 6.0 & 5.11 & 7.8 & 10.2 & $<1.7$ & $<1.0$ & 1.3 & 4.2 & $<1.0$ & 6.8 & 9.8 & 0.4 & 66 & 190 & na & 1.1 & 17.2 \\
\hline Rabbit Ears 2, Colo. & $3 / 28 / 2007$ & E & -5.9 & 6.1 & 5.15 & 7.1 & 6.4 & $<1.7$ & 1.0 & 0.9 & 4.7 & $<1.0$ & 7.2 & 11.1 & 0.5 & 65 & 191 & na & 1.1 & 4.8 \\
\hline
\end{tabular}


E, depth-integrated environmental; ANC, acid neutralization capacity; $\mu$ eq/L, microequivalents per liter; $\mu \mathrm{S} / \mathrm{cm}$, microsiemens per centimeter; $\mathrm{SC}$, specific conductance at $25^{\circ} \mathrm{C}$; $\mathrm{pH}$ values in standard units; $\mathrm{H}$, hydrogen; Ca, calcium; $\mathrm{Mg}$, magnesium; $\mathrm{Na}$, sodium; K, potassium; $\mathrm{NH}_{4}$, ammonium; $\mathrm{Cl}$, chloride; $\mathrm{SO}_{4}$, sulfate; $\mathrm{NO}_{3}$, nitrate; DOC, dissolved organic carbon; $\mathrm{mg} / \mathrm{L}$, milligrams per liter; $\mathrm{SWE}$, snow/water equivalent; cm, centimeters; NADP, National Atmospheric Deposition Program; $\delta^{34} \mathrm{~S}$, stable sulfur isotope ratio $\left({ }^{34} \mathrm{~S} /{ }^{32} \mathrm{~S}\right) ; \mathrm{Hg}$, mercury; ng/L, nanograms per liter; na, not analyzed; <, below reporting limits; \%, percent]

\begin{tabular}{|c|c|c|c|c|c|c|c|c|c|c|c|c|c|c|c|c|c|c|c|c|}
\hline Site name & $\begin{array}{l}\text { Sample } \\
\text { date }\end{array}$ & $\begin{array}{l}\text { Sample } \\
\text { type }\end{array}$ & $\begin{array}{c}\text { ANC } \\
\text { ( } \mu \text { eq/L) }\end{array}$ & $\underset{(\mu \mathrm{S} / \mathrm{cm})}{\mathrm{SC}}$ & $\mathrm{pH}$ & 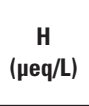 & 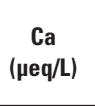 & $\underset{(\mu \mathrm{eq} / \mathrm{L})}{\mathrm{Mg}}$ & $\begin{array}{c}\mathrm{Na} \\
(\mu \mathrm{eq} / \mathrm{L})\end{array}$ & $\begin{array}{c}K \\
(\mu e q / L)\end{array}$ & $\begin{array}{c}\mathrm{NH}_{4} \\
\text { ( } \mu \mathrm{eq} / \mathrm{L} \text { ) }\end{array}$ & $\begin{array}{c}\mathrm{Cl} \\
(\mu \mathrm{eq} / \mathrm{L})\end{array}$ & $\begin{array}{c}\mathrm{SO}_{4} \\
(\mu \mathrm{eq} / \mathrm{L})\end{array}$ & $\begin{array}{c}\mathrm{NO}_{3} \\
(\mu \mathrm{eq} / \mathrm{L})\end{array}$ & $\begin{array}{l}\text { DOC } \\
\text { (mg/L) }\end{array}$ & $\begin{array}{l}\text { SWE } \\
\text { (cm) }\end{array}$ & $\begin{array}{l}\text { Snow } \\
\text { depth } \\
\text { (cm) }\end{array}$ & $\begin{array}{c}\delta^{34} \mathbf{S} \\
\text { (per mil) }\end{array}$ & $\underset{\text { (ng/L) }}{\mathrm{Hg}}$ & $\begin{array}{c}\text { lonic } \\
\text { balance } \\
(\%)\end{array}$ \\
\hline $\begin{array}{l}\text { Red Mountain, } \\
\text { Mont. }\end{array}$ & 3/3/2007 & E & 2.6 & 3.6 & 5.55 & 2.8 & 5.8 & $<1.7$ & 1.1 & 0.5 & 3.2 & $<1.0$ & 3.6 & 4.2 & 0.4 & 40 & 146 & na & 2.4 & 12.3 \\
\hline $\begin{array}{l}\text { Red Mountain Pass, } \\
\text { Colo. }\end{array}$ & $3 / 27 / 2007$ & E & 15.7 & 5.3 & 6.28 & 0.5 & 23.5 & 3.2 & 1.8 & 3.4 & 3.3 & 1.9 & 6.4 & 9.8 & 0.6 & 56 & 178 & na & 4.6 & 2.8 \\
\hline $\begin{array}{l}\text { Rendezvous Moun- } \\
\text { tain, Wyo. }\end{array}$ & $3 / 13 / 2007$ & E & 14.1 & 4.0 & 6.18 & 0.7 & 8.8 & 4.5 & 1.4 & $<0.4$ & 5.6 & $<1.0$ & 4.5 & 5.7 & $<0.4$ & 54 & 183 & na & 1.9 & -7.3 \\
\hline $\begin{array}{l}\text { Ripple Creek NADP, } \\
\text { Colo. }\end{array}$ & $3 / 27 / 2007$ & E & 13.4 & 4.7 & 6.29 & 0.5 & 19.6 & 3.3 & 1.1 & 0.6 & 3.6 & 1.2 & 3.9 & 8.7 & 0.9 & 31 & 85 & na & 2.1 & 2.6 \\
\hline $\begin{array}{l}\text { Slumgullion Pass, } \\
\text { Colo. }\end{array}$ & $3 / 29 / 2007$ & E & 8.5 & 4.0 & 5.99 & 1.0 & 18.8 & 2.2 & 1.5 & 4.3 & $<1.0$ & 1.9 & 5.1 & 6.5 & 0.9 & 24 & 98 & na & 4.9 & 11.4 \\
\hline Snow Bowl, Mont. & $3 / 4 / 2007$ & $\mathrm{E}$ & -3.5 & 4.8 & 5.25 & 5.6 & $<3.1$ & $<1.7$ & 1.4 & 1.1 & $<1.0$ & 2.1 & 3.6 & 3.7 & 0.7 & 66 & 228 & na & 11.0 & -6.5 \\
\hline South Pass, Wyo. & $3 / 19 / 2007$ & E & 2.5 & 5.3 & 5.60 & 2.5 & 13.6 & 3.0 & 2.6 & 5.2 & 2.8 & 1.8 & 8.2 & 11.0 & 1.8 & 21 & 70 & na & 5.2 & 11.8 \\
\hline Spring Gulch, Mont. & 2/23/2007 & E & 1.0 & 4.1 & 5.60 & 2.5 & 5.9 & $<1.7$ & $<1.0$ & 1.4 & 3.7 & $<1.0$ & 3.1 & 7.9 & 0.5 & 15 & 70 & na & 9.8 & 5.6 \\
\hline Sunlight Peak, Colo. & 3/29/2007 & E & 2.8 & 4.9 & 5.68 & 2.1 & 20.8 & 2.2 & 1.6 & 1.0 & 3.9 & 1.2 & 5.9 & 10.4 & 0.5 & 50 & 170 & na & 1.8 & 21.6 \\
\hline Sylvan Lake, Wyo. & 2/20/2007 & $\mathrm{E}$ & -1.2 & 3.9 & 5.51 & 3.1 & 6.4 & $<1.7$ & 1.2 & 0.7 & 4.2 & $<1.0$ & 4.4 & 5.4 & 0.4 & 36 & 145 & na & 2.9 & 22.9 \\
\hline $\begin{array}{l}\text { Sylvan Lake (in } \\
\text { road), Wyo. }\end{array}$ & 2/20/2007 & E & -0.3 & 4.0 & 5.55 & 2.8 & 5.2 & $<1.7$ & 1.3 & $<0.4$ & 6.0 & 1.2 & 4.8 & 6.0 & $<0.4$ & na & 110 & na & 1.1 & 12.5 \\
\hline $\begin{array}{l}\text { Taos Ski Valley, } \\
\text { N. Mex. }\end{array}$ & $3 / 25 / 2007$ & E & 13.0 & 6.9 & 6.10 & 0.8 & 16.9 & 14.6 & 2.3 & 6.5 & 2.0 & 2.0 & 11.4 & 11.5 & 1.5 & 45 & 142 & na & 13.2 & 6.3 \\
\hline Teton Pass, Wyo. & $3 / 15 / 2007$ & E & 10.9 & 4.8 & 6.04 & 0.9 & 8.8 & 3.0 & 2.0 & 2.6 & 6.8 & 1.8 & 5.1 & 6.9 & 0.8 & 51 & 161 & na & 5.9 & -1.1 \\
\hline $\begin{array}{l}\text { Togwotee Pass, } \\
\text { Wyo. }\end{array}$ & $3 / 12 / 2007$ & E & 5.1 & 4.4 & 5.60 & 2.5 & 7.2 & 2.3 & $<1.0$ & 5.4 & 2.7 & 1.5 & 3.6 & 5.1 & 1.3 & 30 & 122 & na & 7.8 & 13.6 \\
\hline $\begin{array}{l}\text { Twenty-one Mile, } \\
\text { Mont. }\end{array}$ & 2/22/2007 & E & 7.3 & 4.9 & 5.96 & 1.1 & 6.1 & $<1.7$ & 2.0 & 0.5 & 8.8 & 2.2 & 4.2 & 7.6 & 0.4 & 31 & 115 & na & 9.6 & -7.0 \\
\hline $\begin{array}{l}\text { University Camp, } \\
\text { Colo. }\end{array}$ & $4 / 2 / 2007$ & E & -3.6 & 5.5 & 5.29 & 5.1 & 6.6 & $<1.7$ & $<1.0$ & 2.1 & 5.1 & $<1.0$ & 6.4 & 10.5 & 0.9 & 48 & 156 & na & 2.8 & 6.0 \\
\hline $\begin{array}{l}\text { West Yellowstone, } \\
\text { Mont. }\end{array}$ & $2 / 22 / 2007$ & E & 12.1 & 5.2 & 6.12 & 0.8 & 10.8 & $<1.7$ & 2.0 & 0.7 & 10.7 & 1.7 & 5.5 & 9.5 & 0.6 & 20 & 83 & na & 5.0 & -7.1 \\
\hline $\begin{array}{l}\text { West Yellowstone } \\
\text { (in road), Mont. }\end{array}$ & $2 / 22 / 2007$ & E & 17.4 & 7.5 & 6.31 & 0.5 & 15.1 & 3.2 & 11.3 & 1.5 & 16.6 & 11.0 & 8.2 & 9.4 & 1.3 & na & 60 & na & 5.2 & 2.3 \\
\hline Wild Basin, Colo. & $4 / 2 / 2007$ & E & 20.2 & 6.3 & 5.84 & 1.4 & 10.1 & 2.4 & 1.2 & 5.4 & 4.4 & 1.3 & 8.6 & 12.4 & 1.3 & 44 & 164 & na & 13.7 & -25.9 \\
\hline $\begin{array}{l}\text { Wolf Creek Pass, } \\
\text { Colo. }\end{array}$ & $3 / 28 / 2007$ & E & 3.4 & 5.5 & 5.74 & 1.8 & 29.2 & 3.3 & 3.2 & 1.8 & 4.2 & 2.9 & 10.0 & 11.4 & 0.7 & 59 & 179 & na & 9.3 & 22.3 \\
\hline
\end{tabular}


Table 17. Selected chemical and physical data for Rocky Mountain snowpack samples for 2008.

E, depth-integrated environmental; ANC, acid neutralization capacity; $\mu$ eq/L, microequivalents per liter; $\mu \mathrm{S} / \mathrm{cm}$, microsiemens per centimeter; $\mathrm{SC}$, specific conductance at $25^{\circ} \mathrm{C} ; \mathrm{pH}$ values in standard units; $\mathrm{H}$, hydrogen; Ca, calcium; Mg, magnesium; $\mathrm{Na}$, sodium; $\mathrm{K}$, potassium; $\mathrm{NH}_{4}$, ammonium; $\mathrm{Cl}$, chloride; $\mathrm{SO}_{4}$, sulfate; $\mathrm{NO}_{3}$, nitrate; DOC, dissolved organic carbon; mg/L, milligrams per liter; $\mathrm{SWE}$, snow/water equivalent; cm, centimeters; NADP, National Atmospheric Deposition Program; $\delta^{34} \mathrm{~S}$, stable sulfur isotope ratio $\left({ }^{34} \mathrm{~S} /{ }^{2} \mathrm{~S}\right) ; \mathrm{Hg}$, mercury; ng/L, nanograms per liter; na, not analyzed; <, below reporting limits; \%, percent]

\begin{tabular}{|c|c|c|c|c|c|c|c|c|c|c|c|c|c|c|c|c|c|c|c|c|}
\hline Site name & $\begin{array}{l}\text { Sample } \\
\text { date }\end{array}$ & $\begin{array}{c}\text { Sample } \\
\text { type }\end{array}$ & $\begin{array}{c}\text { ANC } \\
\text { (meq/L) }\end{array}$ & $\begin{array}{c}\mathrm{SC} \\
(\mathrm{mS} / \mathrm{cm})\end{array}$ & $\mathrm{pH}$ & $\underset{(\mathrm{meq} / \mathrm{L})}{\mathbf{H}}$ & $\begin{array}{c}\mathrm{Ca} \\
\text { (meq/L) }\end{array}$ & $\underset{\text { (meq/L) }}{\mathrm{Mg}}$ & $\begin{array}{c}\mathrm{Na} \\
\text { (meq/L) }\end{array}$ & $\begin{array}{c}\mathbf{K} \\
\text { (meq/L) }\end{array}$ & $\begin{array}{c}\mathrm{NH}^{4} \\
\text { (meq/L) }\end{array}$ & $\underset{\text { (meq/L) }}{\text { CI }}$ & $\begin{array}{c}\mathrm{SO}_{4} \\
\text { (meq/L) }\end{array}$ & $\begin{array}{c}\mathrm{NO}_{3} \\
\text { (meq/L) }\end{array}$ & $\begin{array}{c}\text { DOC } \\
\text { (mg/L) }\end{array}$ & $\begin{array}{l}\text { SWE } \\
(\mathrm{cm})\end{array}$ & $\begin{array}{l}\text { Snow } \\
\text { depth } \\
\text { (cm) }\end{array}$ & $\begin{array}{c}\delta^{34} \mathrm{~S} \\
\text { (per mil) }\end{array}$ & $\begin{array}{c}\mathrm{Hg} \\
\text { (ng/L) }\end{array}$ & $\begin{array}{c}\text { lonic } \\
\text { balance } \\
(\%)\end{array}$ \\
\hline $\begin{array}{l}\text { Apgar Lookout, } \\
\text { Mont. }\end{array}$ & 4/13/2008 & $\mathrm{E}$ & -2.7 & 4.5 & 5.29 & 5.1 & 4.5 & $<1.7$ & 1.8 & 0.6 & 6.3 & 1.3 & 4.4 & 6.0 & $<0.4$ & 72.9 & 207 & na & 1.5 & 22.0 \\
\hline $\begin{array}{l}\text { Banner Summit, } \\
\text { Idaho }\end{array}$ & $3 / 6 / 2008$ & E & -3.4 & 2.7 & 5.50 & 3.2 & 4.0 & $<1.7$ & 2.2 & 0.6 & 1.9 & $<1.0$ & 1.7 & 2.7 & $<0.4$ & 54.2 & 183 & na & 0.6 & 46.0 \\
\hline $\begin{array}{l}\text { Berthoud Pass, } \\
\text { Colo. }\end{array}$ & $4 / 3 / 2008$ & $\mathrm{E}$ & 0.0 & 8.1 & 5.08 & 8.3 & 14.5 & 2.5 & 3.1 & 2.3 & 2.7 & 2.8 & 4.7 & 9.7 & 0.8 & 59.2 & 203 & na & 4.2 & $(32.0)$ \\
\hline $\begin{array}{l}\text { Big Mountain, } \\
\text { Mont. }\end{array}$ & $2 / 29 / 2008$ & $\mathrm{E}$ & -4.7 & 5.2 & 5.12 & 7.6 & 4.0 & $<1.7$ & $<1.0$ & $<0.4$ & 2.2 & $<1.0$ & 2.4 & 4.0 & $<0.4$ & 96.9 & 297 & na & 0.9 & 36.6 \\
\hline Big Sky, Mont. & $3 / 5 / 2008$ & E & 6.0 & 4.0 & 5.94 & 1.1 & 13.5 & $<1.7$ & 1.3 & 0.8 & 8.0 & 1.1 & 5.1 & 7.2 & 0.5 & 40.6 & 150 & na & 1.4 & 12.0 \\
\hline $\begin{array}{l}\text { Brooklyn Lake, } \\
\text { Wyo. }\end{array}$ & $3 / 27 / 2008$ & E & -3.8 & 5.5 & 5.30 & 5.0 & 10.5 & $<1.7$ & 2.4 & 0.8 & 4.1 & 2.4 & 6.8 & 9.8 & 0.6 & 73.8 & 237 & na & 3.4 & 9.1 \\
\hline Brumley, Colo. & $3 / 25 / 2008$ & E & 1.0 & 7.5 & 5.08 & 8.3 & 12.0 & $<1.7$ & 3.1 & 0.6 & 2.4 & 1.6 & 4.3 & 7.3 & $<0.4$ & 48.0 & 164 & na & 2.5 & 30.0 \\
\hline Buffalo Pass, Colo. & $4 / 1 / 2008$ & E & -3.5 & 7.2 & 5.10 & 7.9 & 11.0 & $<1.7$ & 2.3 & 0.6 & 4.2 & 1.1 & 6.8 & 10.2 & $<0.4$ & 121.6 & 384 & na & 1.6 & 17.9 \\
\hline Cameron Pass, Colo. & $3 / 23 / 2008$ & $\mathrm{E}$ & -4.4 & 7.9 & 5.02 & 9.5 & 9.0 & $<1.7$ & 6.3 & 1.6 & 3.5 & 6.8 & 4.2 & 8.2 & 0.7 & 50.0 & 167 & na & 5.7 & 21.8 \\
\hline Canyon, Wyo. & $2 / 20 / 2008$ & $\mathrm{E}$ & -0.6 & 3.1 & 5.61 & 2.5 & 4.0 & $<1.7$ & $<1.0$ & $<0.4$ & 5.7 & $<1.0$ & 1.9 & 5.5 & $<0.4$ & 35.6 & 133 & na & 2.7 & 24.5 \\
\hline $\begin{array}{l}\text { Chief Joseph Pass, } \\
\text { Mont. }\end{array}$ & $3 / 1 / 2008$ & E & -4.1 & 6.6 & 5.01 & 9.8 & 4.5 & $<1.7$ & $<1.0$ & 0.6 & 2.3 & $<1.0$ & 2.0 & 3.4 & 0.5 & 61.7 & 202 & na & 1.9 & 52.2 \\
\hline Daisy Pass, Mont. & $2 / 26 / 2008$ & E & -4.3 & 5.5 & 5.18 & 6.6 & 5.0 & $<1.7$ & $<1.0$ & 0.4 & 4.6 & $<1.0$ & 2.6 & 4.8 & $<0.4$ & 90.5 & 300 & na & 0.7 & 38.3 \\
\hline Divide Peak, Wyo. & $3 / 26 / 2008$ & $\mathrm{E}$ & -4.0 & 6.8 & 5.25 & 5.6 & 16.0 & 2.5 & 3.0 & 1.2 & 5.0 & 1.6 & 7.3 & 14.3 & 0.6 & 66.0 & 187 & na & 1.9 & 17.9 \\
\hline Dry Lake, Colo. & $3 / 4 / 2008$ & E & -14.9 & 8.5 & 4.98 & 10.5 & 8.0 & $<1.7$ & 1.1 & 0.6 & 3.2 & 1.0 & 5.1 & 15.9 & $<0.4$ & 65.0 & 193 & na & 1.1 & 3.1 \\
\hline $\begin{array}{l}\text { Dunckley Pass, } \\
\text { Colo. }\end{array}$ & $4 / 2 / 2008$ & E & 4.2 & 6.2 & 5.42 & 3.8 & 17.5 & 3.3 & 3.9 & 2.5 & 1.9 & 3.4 & 6.8 & 10.3 & 1.1 & 66.2 & 202 & na & 1.9 & 14.2 \\
\hline Elk River, Colo. & $3 / 21 / 2008$ & $\mathrm{E}$ & -1.5 & 5.7 & 5.27 & 5.4 & 12.5 & $<1.7$ & $<1.0$ & 0.8 & 4.5 & $<1.0$ & 4.0 & 11.5 & 0.4 & 51.2 & 157 & na & 1.7 & 19.9 \\
\hline Elkhart Park, Wyo. & $3 / 18 / 2008$ & $\mathrm{E}$ & -8.5 & 6.1 & 5.10 & 7.9 & 6.0 & $<1.7$ & $<1.0$ & 0.8 & 5.3 & $<1.0$ & 4.1 & 7.3 & 0.8 & 26.6 & 106 & na & 1.1 & 27.4 \\
\hline $\begin{array}{l}\text { Four Mile Meadow, } \\
\text { Wyo. }\end{array}$ & $3 / 20 / 2008$ & $\mathrm{E}$ & -0.9 & 3.4 & 5.58 & 2.6 & 4.5 & $<1.7$ & $<1.0$ & $<0.4$ & 6.1 & 1.0 & 2.2 & 6.2 & $<0.4$ & 28.4 & 112 & na & 1.3 & 16.8 \\
\hline Fremont Pass, Colo. & $4 / 5 / 2008$ & E & 3.5 & 4.0 & 5.58 & 2.6 & 12.0 & $<1.7$ & 1.7 & 1.6 & 2.7 & 1.4 & 3.4 & 7.3 & 0.7 & 48.4 & 180 & na & 0.5 & 13.8 \\
\hline $\begin{array}{l}\text { Galena Summit, } \\
\text { Idaho }\end{array}$ & $3 / 6 / 2008$ & $\mathrm{E}$ & 3.0 & 2.5 & 5.60 & 2.5 & 4.0 & $<1.7$ & $<1.0$ & $<0.4$ & 2.0 & $<1.0$ & 1.2 & 2.5 & $<0.4$ & 54.7 & 194 & na & $<0.4$ & 11.8 \\
\hline $\begin{array}{l}\text { Garnet Canyon, } \\
\text { Wyo. }\end{array}$ & $3 / 17 / 2008$ & $\mathrm{E}$ & -7.0 & 5.6 & 5.16 & 6.9 & 5.0 & $<1.7$ & 1.1 & 0.4 & 5.0 & $<1.0$ & 2.6 & 5.9 & $<0.4$ & 69.8 & 218 & na & 1.0 & 36.8 \\
\hline Grand Mesa, Colo. & $4 / 2 / 2008$ & $\mathrm{E}$ & 6.5 & 5.0 & 5.79 & 1.6 & 19.5 & 3.3 & 4.3 & 1.1 & 3.5 & 2.3 & 7.1 & 8.3 & 0.5 & 65.9 & 197 & na & 3.5 & 15.8 \\
\hline Granite Pass, Mont. & $3 / 3 / 2008$ & E & -5.6 & 3.6 & 5.28 & 5.2 & $<3.1$ & $<1.7$ & 1.5 & 0.6 & 1.1 & 1.0 & 1.9 & 2.6 & $<0.4$ & 85.4 & 257 & na & 5.0 & 20.9 \\
\hline
\end{tabular}


E, depth-integrated environmental; $\mathrm{ANC}$, acid neutralization capacity; $\mu \mathrm{eq} / \mathrm{L}$, microequivalents per liter; $\mu \mathrm{S} / \mathrm{cm}$, microsiemens per centimeter; $\mathrm{SC}$, specific conductance at $25^{\circ} \mathrm{C}$; $\mathrm{pH}$ values in standard units; $\mathrm{H}$, hydrogen; Ca, calcium; $\mathrm{Mg}$, magnesium; $\mathrm{Na}$, sodium; K, potassium; $\mathrm{NH}_{4}$, ammonium; $\mathrm{Cl}$, chloride; $\mathrm{SO}_{4}$, sulfate; $\mathrm{NO}_{3}$, nitrate; DOC, dissolved organic carbon; $\mathrm{mg} / \mathrm{L}$, milligrams per liter; $\mathrm{SWE}$, snow/water equivalent; cm, centimeters; NADP, National Atmospheric Deposition Program; $\delta^{34} \mathrm{~S}$, stable sulfur isotope ratio $\left({ }^{34} \mathrm{~S} /{ }^{2} \mathrm{~S}\right) ; \mathrm{Hg}$, mercury; ng $/ \mathrm{L}$, nanograms per liter; na, not analyzed; <, below reporting limits; \%, percent]

\begin{tabular}{|c|c|c|c|c|c|c|c|c|c|c|c|c|c|c|c|c|c|c|c|c|}
\hline Site name & $\begin{array}{l}\text { Sample } \\
\text { date }\end{array}$ & $\begin{array}{l}\text { Sample } \\
\text { type }\end{array}$ & $\begin{array}{c}\text { ANC } \\
\text { (meq/L) }\end{array}$ & $\begin{array}{c}\mathrm{SC} \\
(\mathrm{mS} / \mathrm{cm})\end{array}$ & $\mathrm{pH}$ & $\underset{\text { (meq/L) }}{\mathbf{H}}$ & $\begin{array}{c}\mathrm{Ca} \\
\text { (meq/L) }\end{array}$ & $\underset{(m e q / L)}{M g}$ & $\underset{(\mathrm{meq} / \mathrm{L})}{\mathrm{Na}}$ & $\begin{array}{c}K \\
\text { (meq/L) }\end{array}$ & $\begin{array}{c}\mathrm{NH}^{4} \\
\text { (meq/L) }\end{array}$ & $\begin{array}{c}\text { CI } \\
\text { (meq/L) }\end{array}$ & $\begin{array}{c}\mathrm{SO}_{4} \\
\text { (meq/L) }\end{array}$ & $\begin{array}{c}\mathrm{NO}_{3} \\
\text { (meq/L) }\end{array}$ & $\begin{array}{l}\text { DOC } \\
\text { (mg/L) }\end{array}$ & $\begin{array}{l}\text { SWE } \\
(\mathrm{cm})\end{array}$ & $\begin{array}{l}\text { Snow } \\
\text { depth } \\
\text { (cm) }\end{array}$ & $\begin{array}{c}\delta^{34} \mathbf{S} \\
\text { (per mil) }\end{array}$ & $\begin{array}{c}\mathrm{Hg} \\
(\mathrm{ng} / \mathrm{L})\end{array}$ & $\begin{array}{c}\text { Ionic } \\
\text { balance } \\
(\%)\end{array}$ \\
\hline Grizzly Ridge, Utah & $3 / 25 / 2008$ & E & 0.9 & 5.2 & 5.40 & 4.0 & 13.5 & $<1.7$ & 1.2 & 0.8 & 3.5 & $<1.0$ & 4.9 & 8.5 & 0.5 & 30.3 & 99 & na & 6.6 & 23.3 \\
\hline $\begin{array}{l}\text { Gypsum Creek, } \\
\text { Wyo. }\end{array}$ & $3 / 18 / 2008$ & E & -3.8 & 4.5 & 5.33 & 4.7 & 6.5 & $<1.7$ & 1.1 & 1.7 & 4.3 & 1.2 & 3.0 & 7.8 & 0.8 & 23.3 & 98 & na & 1.9 & 20.8 \\
\hline Hopewell, N. Mex. & $3 / 29 / 2008$ & E & -4.8 & 5.2 & 5.22 & 6.0 & 8.0 & $<1.7$ & $<1.0$ & 1.1 & 3.4 & $<1.0$ & 4.5 & 6.8 & 0.5 & 66.6 & 177 & na & 6.4 & 24.2 \\
\hline Kings Hill, Mont. & $2 / 23 / 2008$ & E & 1.1 & 4.1 & 5.60 & 2.5 & 12.0 & $<1.7$ & $<1.0$ & 0.6 & 6.0 & 1.0 & 3.9 & 6.2 & 0.7 & 34.8 & 112 & na & 2.9 & 26.7 \\
\hline Lake Fork, Utah & $3 / 24 / 2008$ & E & 5.7 & 5.2 & 5.37 & 4.3 & 15.5 & $<1.7$ & 1.7 & 0.4 & 2.1 & 1.1 & 3.5 & 5.5 & $<0.4$ & 32.9 & 117 & na & 0.7 & 20.6 \\
\hline Lake Irene, Colo. & 4/10/2008 & E & -1.5 & 4.4 & 5.43 & 3.7 & 8.5 & $<1.7$ & 2.0 & 0.6 & 4.7 & 1.2 & 5.6 & 9.6 & 0.5 & 66.2 & 219 & na & 2.6 & 8.6 \\
\hline $\begin{array}{l}\text { Lewis Lake Divide, } \\
\text { Wyo. }\end{array}$ & $2 / 18 / 2008$ & E & 0.0 & 3.2 & 5.70 & 2.0 & 4.5 & $<1.7$ & 1.1 & $<0.4$ & 7.7 & 1.1 & 2.7 & 5.2 & $<0.4$ & 77.6 & 250 & na & 0.5 & 25.9 \\
\hline Lionshead, Mont. & $2 / 27 / 2008$ & E & -0.6 & 4.9 & 5.26 & 5.5 & 6.0 & $<1.7$ & 1.1 & 0.8 & 12.0 & 1.2 & 4.1 & 8.9 & 0.5 & 62.1 & 202 & na & 2.1 & 28.3 \\
\hline $\begin{array}{l}\text { Loch Vale Forest, } \\
\text { Colo. }\end{array}$ & $4 / 8 / 2008$ & E & 0.8 & 4.6 & 5.60 & 2.5 & 11.5 & $<1.7$ & 3.7 & 1.0 & 5.4 & 1.8 & 6.8 & 10.7 & 0.6 & 97.5 & 310 & na & 4.3 & 9.0 \\
\hline $\begin{array}{l}\text { Loch Vale Meadow, } \\
\text { Colo. }\end{array}$ & $4 / 8 / 2008$ & E & 8.2 & 5.0 & 5.96 & 1.1 & 16.5 & 2.5 & 7.0 & 0.6 & 7.4 & 2.9 & 9.6 & 11.2 & 0.4 & 75.9 & 245 & na & 2.5 & 4.8 \\
\hline $\begin{array}{l}\text { Loveland Pass, } \\
\text { Colo. }\end{array}$ & $4 / 11 / 2008$ & E & 2.6 & 3.5 & 5.60 & 2.5 & 9.0 & $<1.7$ & 2.7 & $<0.4$ & 3.3 & 2.9 & 3.4 & 6.5 & $<0.4$ & 61.4 & 212 & na & 1.3 & 6.4 \\
\hline Molas Lake, Colo. & $3 / 30 / 2008$ & E & 2.2 & 4.2 & 5.68 & 2.1 & 4.0 & $<1.7$ & $<1.0$ & $<0.4$ & 1.4 & $<1.0$ & 2.0 & 5.1 & $<0.4$ & 64.3 & 208 & na & 1.0 & -10.7 \\
\hline Monarch Pass, Colo. & $3 / 26 / 2008$ & E & -6.4 & 6.2 & 5.02 & 9.5 & 18.5 & 3.3 & 4.9 & 1.0 & 2.4 & 2.4 & 6.1 & 5.7 & $<0.4$ & 53.2 & 172 & na & 3.6 & $(47.2)$ \\
\hline $\begin{array}{l}\text { Mount Belmont, } \\
\text { Mont. }\end{array}$ & $2 / 22 / 2008$ & E & 1.7 & 5.1 & 5.32 & 4.8 & 9.5 & $<1.7$ & 1.5 & 1.6 & 6.1 & 1.6 & 4.3 & 6.6 & 1.5 & 21.4 & 74 & na & 5.2 & 24.7 \\
\hline Music Pass, Colo. & $3 / 28 / 2008$ & E & -3.9 & 6.0 & 5.38 & 4.2 & 7.0 & $<1.7$ & $<1.0$ & 1.1 & 3.6 & $<1.0$ & 3.5 & 6.8 & 0.4 & 73.3 & 204 & na & 2.5 & 21.4 \\
\hline Noisy Basin, Mont. & $2 / 28 / 2008$ & E & -4.7 & 4.7 & 5.23 & 5.9 & 5.0 & $<1.7$ & 1.5 & 0.8 & 4.5 & 1.5 & 4.8 & 5.3 & 0.4 & 86.6 & 303 & na & 1.3 & 20.8 \\
\hline Old Battle, Wyo. & $3 / 26 / 2008$ & E & -5.3 & 6.1 & 5.16 & 6.9 & 10.0 & $<1.7$ & 1.9 & 1.6 & 2.8 & 1.4 & 6.3 & 11.5 & 0.7 & 77.7 & 221 & na & 3.4 & 9.4 \\
\hline $\begin{array}{l}\text { Old Faithful (in } \\
\text { road), Wyo. }\end{array}$ & $2 / 20 / 2008$ & E & 4.4 & 4.7 & 5.88 & 1.3 & 9.5 & $<1.7$ & 4.1 & 1.8 & 14.0 & 5.6 & 4.0 & 7.7 & 0.9 & na & 79 & na & 4.6 & 17.2 \\
\hline $\begin{array}{l}\text { Old Faithful Fire } \\
\text { Road, Wyo. }\end{array}$ & 2/20/2008 & E & -0.5 & 3.4 & 5.61 & 2.5 & 4.5 & $<1.7$ & 1.7 & $<0.4$ & 8.1 & 1.8 & 2.6 & 6.7 & $<0.4$ & 32.3 & 111 & na & 0.4 & 20.4 \\
\hline Rabbit Ears 1, Colo. & $3 / 28 / 2008$ & E & -5.3 & 5.2 & 5.36 & 4.4 & 11.5 & $<1.7$ & 3.1 & 0.7 & 3.8 & 1.4 & 7.7 & 10.4 & 0.5 & 87.9 & 263 & na & 2.4 & 9.3 \\
\hline Rabbit Ears 2, Colo. & $3 / 28 / 2008$ & E & -3.8 & 5.8 & 5.26 & 5.5 & 11.0 & $<1.7$ & 2.7 & 0.7 & 5.3 & 1.9 & 7.4 & 11.3 & 0.4 & 84.5 & 253 & na & 1.4 & 10.0 \\
\hline $\begin{array}{l}\text { Red Mountain, } \\
\text { Mont. }\end{array}$ & $3 / 4 / 2008$ & E & 1.9 & 3.7 & 5.62 & 2.4 & 11.0 & $<1.7$ & $<1.0$ & 1.1 & 6.5 & 1.1 & 3.4 & 6.0 & 0.8 & 48.1 & 169 & na & 2.9 & 25.7 \\
\hline $\begin{array}{l}\text { Red Mountain Pass, } \\
\text { Colo. }\end{array}$ & $3 / 31 / 2008$ & E & 17.0 & 6.0 & 5.51 & 3.1 & 24.0 & 2.5 & 1.7 & 1.0 & 3.7 & 1.3 & 4.1 & 7.0 & 0.4 & 89.9 & 274 & na & 4.1 & 10.1 \\
\hline
\end{tabular}


Table 17. Selected chemical and physical data for Rocky Mountain snowpack samples for 2008.—Continued

$\mathrm{E}$, depth-integrated environmental; ANC, acid neutralization capacity; $\mu \mathrm{eq} / \mathrm{L}$, microequivalents per liter; $\mu \mathrm{S} / \mathrm{cm}$, microsiemens per centimeter; $\mathrm{SC}$, specific conductance at $25^{\circ} \mathrm{C} ; \mathrm{pH}$ values in standard units; $\mathrm{H}$, hydrogen; Ca, calcium; Mg, magnesium; $\mathrm{Na}$, sodium; $\mathrm{K}$, potassium; $\mathrm{NH}_{4}$, ammonium; $\mathrm{Cl}$, chloride; $\mathrm{SO}_{4}$, sulfate; $\mathrm{NO}_{3}$, nitrate; DOC, dissolved organic carbon; mg/L, milligrams per liter; $\mathrm{SWE}$, snow/water equivalent; cm, centimeters; NADP, National Atmospheric Deposition Program; $\delta^{34} \mathrm{~S}$, stable sulfur isotope ratio $\left({ }^{34} \mathrm{~S} /{ }^{32} \mathrm{~S}\right) ; \mathrm{Hg}$, mercury; ng/L, nanograms per liter; na, not analyzed; <, below reporting limits; \%, percent]

\begin{tabular}{|c|c|c|c|c|c|c|c|c|c|c|c|c|c|c|c|c|c|c|c|c|}
\hline Site name & $\begin{array}{l}\text { Sample } \\
\text { date }\end{array}$ & $\begin{array}{c}\text { Sample } \\
\text { type }\end{array}$ & $\begin{array}{c}\text { ANC } \\
\text { (meq/L) }\end{array}$ & $\begin{array}{c}\mathrm{SC} \\
(\mathrm{mS} / \mathrm{cm})\end{array}$ & $\mathrm{pH}$ & $\begin{array}{c}\text { H } \\
\text { (meq/L) }\end{array}$ & $\underset{\text { (meq/L) }}{\mathrm{Ca}}$ & $\underset{(m e q / L)}{M g}$ & $\underset{\text { (meq/L) }}{\mathrm{Na}}$ & $\underset{(m e q / L)}{K}$ & $\begin{array}{c}\mathrm{NH}^{4} \\
\text { (meq/L) }\end{array}$ & $\underset{(m e q / L)}{C l}$ & $\underset{(\mathrm{meq} / \mathrm{L})}{\mathrm{SO}_{4}}$ & $\begin{array}{c}\mathrm{NO}_{3} \\
(\mathrm{meq} / \mathrm{L})\end{array}$ & $\begin{array}{c}\text { DOC } \\
\text { (mg/L) }\end{array}$ & $\begin{array}{l}\text { SWE } \\
\text { (cm) }\end{array}$ & $\begin{array}{l}\text { Snow } \\
\text { depth } \\
\text { (cm) }\end{array}$ & $\begin{array}{c}\delta^{34} \mathrm{~S} \\
\text { (per mil) }\end{array}$ & $\underset{(\mathrm{ng} / \mathrm{L})}{\mathrm{Hg}}$ & $\begin{array}{c}\text { lonic } \\
\text { balance } \\
(\%)\end{array}$ \\
\hline $\begin{array}{l}\text { Rendezvous Moun- } \\
\text { tain, Wyo. }\end{array}$ & $3 / 19 / 2008$ & E & 1.5 & 5.0 & 5.30 & 5.0 & 5.5 & $<1.7$ & 1.0 & $<0.4$ & 6.6 & $<1.0$ & 3.2 & 4.4 & $<0.4$ & 79.4 & 271 & na & 0.7 & 33.1 \\
\hline $\begin{array}{l}\text { Ripple Creek NADP, } \\
\text { Colo. }\end{array}$ & $4 / 2 / 2008$ & E & 3.2 & 5.5 & 5.41 & 3.9 & 13.0 & $<1.7$ & 3.2 & 0.6 & 4.0 & 1.8 & 4.6 & 9.3 & $<0.4$ & 54.4 & 160 & na & 1.0 & 13.3 \\
\hline $\begin{array}{l}\text { Slumgullion Pass, } \\
\text { Colo. }\end{array}$ & $4 / 1 / 2008$ & E & 5.7 & 4.5 & 5.46 & 3.5 & 13.0 & $<1.7$ & 1.2 & 1.2 & 1.7 & 1.1 & 2.8 & 5.2 & $<0.4$ & 43.3 & 148 & na & 2.9 & 16.4 \\
\hline Snow Bowl, Mont. & $3 / 2 / 2008$ & E & -4.2 & 6.2 & 5.02 & 9.5 & 5.5 & $<1.7$ & 1.2 & 0.7 & 3.5 & 1.2 & 3.1 & 3.4 & 0.5 & 75.2 & 237 & na & 3.9 & 45.2 \\
\hline South Pass, Wyo. & $3 / 24 / 2008$ & E & -4.0 & 4.4 & 5.37 & 4.3 & 9.0 & $<1.7$ & 1.4 & 1.5 & 3.9 & 1.0 & 5.7 & 7.2 & 1.3 & 40.7 & 125 & na & 2.1 & 18.2 \\
\hline Spring Gulch, Mont. & $2 / 22 / 2008$ & E & 9.4 & 4.6 & 5.62 & 2.4 & 17.5 & 2.5 & 1.6 & 2.4 & 6.2 & 1.2 & 4.1 & 6.6 & 0.8 & 12.9 & 50 & na & 4.8 & 21.0 \\
\hline Sunlight Peak, Colo. & $4 / 4 / 2008$ & E & 12.3 & 5.7 & 5.61 & 2.5 & 20.5 & 3.3 & 4.6 & 0.8 & 5.1 & 2.8 & 5.8 & 9.6 & $<0.4$ & 72.8 & 226 & na & 2.2 & 9.4 \\
\hline Sylvan Lake, Wyo. & $2 / 19 / 2008$ & E & 0.2 & 3.2 & 5.63 & 2.3 & 4.0 & $<1.7$ & $<1.0$ & 0.6 & 5.4 & $<1.0$ & 2.7 & 4.8 & 0.4 & 53.4 & 179 & na & 2.6 & 23.0 \\
\hline $\begin{array}{l}\text { Sylvan Lake (in } \\
\text { road), Wyo. }\end{array}$ & $2 / 19 / 2008$ & E & -2.0 & 3.2 & 5.48 & 3.3 & 3.5 & $<1.7$ & $<1.0$ & $<0.4$ & 4.6 & $<1.0$ & 2.0 & 4.5 & $<0.4$ & na & 108 & na & 5.5 & 27.4 \\
\hline $\begin{array}{l}\text { Taos Ski Valley, N. } \\
\text { Mex. }\end{array}$ & $3 / 30 / 2008$ & E & 2.3 & 5.6 & 5.30 & 5.0 & 10.5 & $<1.7$ & 1.5 & 3.4 & 3.9 & 1.3 & 5.7 & 6.4 & 1.1 & 75.9 & 218 & na & 14.0 & 21.5 \\
\hline Teton Pass, Wyo. & $3 / 20 / 2008$ & E & 4.4 & 4.1 & 5.63 & 2.3 & 8.0 & $<1.7$ & 1.6 & 0.8 & 9.7 & 1.6 & 3.8 & 6.6 & $<0.4$ & 63.9 & 222 & na & 1.9 & 15.5 \\
\hline $\begin{array}{l}\text { Togwotee Pass, } \\
\text { Wyo. }\end{array}$ & $3 / 20 / 2008$ & E & 0.3 & 3.1 & 5.69 & 2.0 & 5.5 & $<1.7$ & 1.3 & 0.6 & 5.0 & 1.2 & 2.6 & 4.1 & $<0.4$ & 68.4 & 255 & na & 2.2 & 27.4 \\
\hline $\begin{array}{l}\text { Twenty-one Mile, } \\
\text { Mont. }\end{array}$ & $2 / 21 / 2008$ & E & -3.2 & 5.3 & 5.32 & 4.8 & 4.5 & $<1.7$ & 1.1 & $<0.4$ & 6.8 & 1.5 & 1.7 & 5.8 & 0.4 & 42.5 & 139 & na & 7.6 & 31.3 \\
\hline $\begin{array}{l}\text { University Camp, } \\
\text { Colo. }\end{array}$ & $4 / 9 / 2008$ & E & 4.0 & 5.0 & 5.70 & 2.0 & 14.5 & $<1.7$ & 3.8 & 1.7 & 7.0 & 2.2 & 8.4 & 11.2 & 0.6 & 52.5 & 183 & na & 3.1 & 5.8 \\
\hline $\begin{array}{l}\text { West Yellowstone, } \\
\text { Mont. }\end{array}$ & $2 / 21 / 2008$ & E & -3.5 & 3.9 & 5.57 & 2.7 & 5.5 & $<1.7$ & 1.3 & $<0.4$ & 10.2 & 1.8 & 3.2 & 8.8 & $<0.4$ & 32.7 & 113 & na & 3.8 & 17.6 \\
\hline $\begin{array}{l}\text { West Yellowstone } \\
\text { (in Road), Mont. }\end{array}$ & $2 / 21 / 2008$ & E & 4.2 & 4.2 & 6.04 & 0.9 & 9.5 & $<1.7$ & 2.0 & 0.4 & 13.8 & 2.4 & 3.8 & 9.0 & 0.8 & na & 71 & na & 1.7 & 15.7 \\
\hline $\begin{array}{l}\text { Wolf Creek Pass, } \\
\text { Colo. }\end{array}$ & $4 / 1 / 2008$ & E & -0.8 & 6.1 & 5.23 & 5.9 & 13.0 & $<1.7$ & 1.6 & 0.7 & 3.4 & 1.2 & 4.9 & 8.4 & $<0.4$ & 92.9 & 265 & na & 3.0 & 25.8 \\
\hline
\end{tabular}


["FB" field blank; "LB" laboratory blank; ANC, acid neutralization capacity; $\mu$ eq/L, microequivalents per liter; SC, specific conductance at $25^{\circ} \mathrm{C}$; $\mu \mathrm{S} / \mathrm{cm}$, microsiemens per centimeter; $\mathrm{pH}$ values in

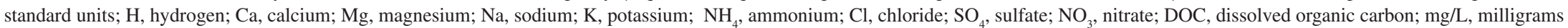
per liter; NADP, National Atmospheric Deposition Program; Hg, mercury; ng/L, nanograms per liter; na, not analyzed; <, below reporting limits; NP, National Park]

\begin{tabular}{|c|c|c|c|c|c|c|c|c|c|c|c|c|c|c|c|c|}
\hline Site name & $\begin{array}{l}\text { Sample } \\
\text { date }\end{array}$ & $\begin{array}{l}\text { Sample } \\
\text { type }\end{array}$ & $\begin{array}{c}\text { ANC } \\
\text { ( } \mu \mathrm{eq} / \mathrm{L})\end{array}$ & $\begin{array}{c}\mathrm{SC} \\
(\mu \mathrm{S} / \mathrm{cm})\end{array}$ & $\mathrm{pH}$ & $\begin{array}{c}H \\
(\mu e q / L)\end{array}$ & 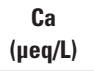 & $\underset{(\mu \mathrm{eq} / \mathrm{L})}{\mathrm{Mg}}$ & $\underset{\text { ( } \mu \mathrm{Neq} / \mathrm{L})}{\mathrm{Na}}$ & $\begin{array}{c}K \\
(\mu e q / L)\end{array}$ & 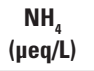 & 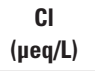 & 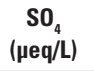 & 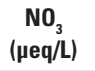 & $\begin{array}{l}\text { DOC } \\
\text { (mg/L) }\end{array}$ & $\underset{(n g / L)}{\mathrm{Hg}}$ \\
\hline \multicolumn{17}{|c|}{1993} \\
\hline Laboratory Blank & 7/1/1993 & LB & -3.5 & 1.6 & 5.51 & 3.1 & $<3.1$ & $<1.7$ & $<1.0$ & $<0.4$ & $<1.0$ & $<1.0$ & $<0.6$ & 0.8 & na & na \\
\hline \multicolumn{17}{|c|}{1995} \\
\hline Laboratory Blank & 7/21/1995 & LB & -1.4 & 1.1 & 5.55 & 2.8 & $<3.1$ & $<1.7$ & na & $<0.4$ & na & na & na & na & na & na \\
\hline Laboratory Blank & 7/21/1995 & LB & -1.2 & 1.2 & 5.56 & 2.8 & $<3.1$ & $<1.7$ & na & $<0.4$ & na & na & na & na & na & na \\
\hline \multicolumn{17}{|c|}{1996} \\
\hline Laboratory Blank & 6/28/1996 & LB & na & na & na & na & $<3.1$ & $<1.7$ & $<1.0$ & na & na & na & na & na & $<0.4$ & na \\
\hline Laboratory Blank & 6/28/1996 & LB & na & na & na & na & $<3.1$ & $<1.7$ & $<1.0$ & na & na & na & na & na & $<0.4$ & na \\
\hline Laboratory Blank & 7/3/1996 & LB & -4.0 & 1.2 & 5.52 & 3.0 & $<3.1$ & $<1.7$ & $<1.0$ & na & na & na & na & na & 2.8 & na \\
\hline Laboratory Blank & 7/3/1996 & LB & -2.5 & 1.2 & 5.59 & 2.6 & $<3.1$ & $<1.7$ & $<1.0$ & na & na & na & na & na & 0.6 & na \\
\hline \multicolumn{17}{|c|}{1997} \\
\hline Laboratory Blank & 6/6/1997 & LB & -3.6 & 1.2 & 5.56 & 2.8 & $<3.1$ & $<1.7$ & $<1.0$ & $<0.4$ & $<1.0$ & $<1.0$ & $<0.6$ & $<0.4$ & 0.4 & na \\
\hline Laboratory Blank & 6/6/1997 & LB & -3.5 & 1.2 & 5.54 & 2.9 & $<3.1$ & $<1.7$ & $<1.0$ & $<0.4$ & $<1.0$ & $<1.0$ & $<0.6$ & $<0.4$ & 0.5 & na \\
\hline Laboratory Blank & 6/9/1997 & LB & -2.8 & 1.1 & 5.51 & 3.1 & $<3.1$ & $<1.7$ & $<1.0$ & na & na & na & na & na & na & na \\
\hline \multicolumn{17}{|c|}{1998} \\
\hline Cement Gulch Divide, Mont. & $3 / 10 / 1998$ & FB & -8.3 & 1.2 & 5.33 & 4.7 & $<3.1$ & $<1.7$ & $<1.0$ & $<0.4$ & $<1.0$ & $<1.0$ & $<0.6$ & $<0.4$ & $<0.4$ & na \\
\hline West Yellowstone, Mont. & $3 / 5 / 1998$ & FB & -5.7 & 1.7 & 5.47 & 3.4 & $<3.1$ & $<1.7$ & $<1.0$ & $<0.4$ & $<1.0$ & $<1.0$ & 1.0 & $<0.4$ & $<0.4$ & na \\
\hline Willow Creek, Wyo. & $3 / 25 / 1998$ & FB & -6.0 & 1.5 & 5.24 & 5.8 & $<3.1$ & $<1.7$ & $<1.0$ & $<0.4$ & $<1.0$ & $<1.0$ & 0.8 & $<0.4$ & $<0.4$ & na \\
\hline Laboratory Blank & $6 / 8 / 1998$ & LB & na & 14.9 & na & na & $<3.1$ & $<1.7$ & 1.7 & $<0.4$ & $<1.0$ & $<1.0$ & 1.7 & $<0.4$ & 5.4 & na \\
\hline Laboratory Blank $^{1}$ & 6/10/1998 & LB & -58.0 & 18.5 & 4.21 & 61.7 & $<3.1$ & $<1.7$ & 2.2 & $<0.4$ & $<1.0$ & $<1.0$ & 0.8 & $<0.4$ & 2.4 & na \\
\hline \multicolumn{17}{|c|}{1999} \\
\hline Cameron Pass, Colo. & $4 / 8 / 1999$ & FB & -3.6 & 3.2 & 5.44 & 3.6 & $<3.1$ & $<1.7$ & $<1.0$ & $<0.4$ & $<1.0$ & $<1.0$ & $<0.6$ & $<0.4$ & $<0.4$ & na \\
\hline Deadman Pass, Colo. & $3 / 23 / 1999$ & FB & -4.7 & 1.6 & 5.41 & 3.9 & $<3.1$ & $<1.7$ & $<1.0$ & $<0.4$ & $<1.0$ & $<1.0$ & $<0.6$ & $<0.4$ & 0.8 & na \\
\hline E7 (Glacier NP), Mont. & 3/31/1999 & FB & -3.4 & 2.1 & 5.35 & 4.5 & $<3.1$ & $<1.7$ & $<1.0$ & 0.5 & $<1.0$ & $<1.0$ & $<0.6$ & $<0.4$ & 1.1 & na \\
\hline Granite Park, Mont. & $3 / 31 / 1999$ & FB & -1.9 & 1.8 & 5.53 & 3.0 & $<3.1$ & $<1.7$ & 1.3 & 0.9 & $<1.0$ & 1.2 & 0.7 & $<0.4$ & 1.1 & na \\
\hline Rabbit Ears 1, Colo. & 3/30/1999 & FB & -5.7 & 1.7 & 5.29 & 5.1 & $<3.1$ & $<1.7$ & $<1.0$ & $<0.4$ & $<1.0$ & $<1.0$ & $<0.6$ & $<0.4$ & 0.7 & na \\
\hline Sunlight Creek, Mont. & 3/9/1999 & FB & -5.6 & 4.0 & 5.14 & 7.2 & $<3.1$ & $<1.7$ & $<1.0$ & $<0.4$ & $<1.0$ & $<1.0$ & 0.8 & $<0.4$ & 0.6 & na \\
\hline $\begin{array}{l}\text { West Yellowstone (in road), } \\
\text { Mont. }\end{array}$ & 3/6/1999 & FB & -4.1 & 3.6 & 5.36 & 4.4 & $<3.1$ & $<1.7$ & $<1.0$ & $<0.4$ & $<1.0$ & $<1.0$ & $<0.6$ & 0.7 & 0.5 & na \\
\hline West Yellowstone, Mont. & 3/6/1999 & FB & -4.9 & 4.3 & 5.24 & 5.8 & $<3.1$ & $<1.7$ & $<1.0$ & $<0.4$ & $<1.0$ & $<1.0$ & $<0.6$ & 0.7 & 0.5 & na \\
\hline Laboratory Blank & 6/9/1999 & LB & -3.3 & 1.1 & 5.51 & 3.1 & $<3.1$ & $<1.7$ & $<1.0$ & $<0.4$ & $<1.0$ & $<1.0$ & $<0.6$ & $<0.4$ & $<0.4$ & na \\
\hline Laboratory Blank & 6/9/1999 & LB & -3.7 & 1.3 & 5.50 & 3.2 & $<3.1$ & $<1.7$ & $<1.0$ & $<0.4$ & $<1.0$ & $<1.0$ & $<0.6$ & $<0.4$ & $<0.4$ & na \\
\hline
\end{tabular}


Table 18. Quality-assurance data: selected chemical concentrations in blank samples for 1993-2008.-Continued

[["FB" field blank; "LB" laboratory blank; ANC, acid neutralization capacity; $\mu$ eq/L, microequivalents per liter; SC, specific conductance at $25^{\circ} \mathrm{C} ; \mu \mathrm{S} / \mathrm{cm}$, microsiemens per centimeter; $\mathrm{pH}$ values in standard units; $\mathrm{H}$, hydrogen; $\mathrm{Ca}$, calcium; $\mathrm{Mg}$, magnesium; $\mathrm{Na}$, sodium; $\mathrm{K}$, potassium; $\mathrm{NH}_{4}$, ammonium; $\mathrm{Cl}$, chloride; $\mathrm{SO}_{4}$, sulfate; $\mathrm{NO}_{3}$, nitrate; $\mathrm{DOC}$, dissolved organic carbon; $\mathrm{mg} / \mathrm{L}$, milligrams per liter; NADP, National Atmospheric Deposition Program; Hg, mercury; ng/L, nanograms per liter; na, not analyzed; <, below reporting limits; NP, National Park]

\begin{tabular}{|c|c|c|c|c|c|c|c|c|c|c|c|c|c|c|c|c|}
\hline Site name & $\begin{array}{l}\text { Sample } \\
\text { date }\end{array}$ & $\begin{array}{c}\text { Sample } \\
\text { type }\end{array}$ & $\begin{array}{c}\text { ANC } \\
(\mu \mathrm{eq} / \mathrm{L})\end{array}$ & $\begin{array}{c}S C \\
(\mu \mathrm{S} / \mathrm{cm})\end{array}$ & $\mathrm{pH}$ & $\begin{array}{c}H \\
(\mu e q / L)\end{array}$ & $\underset{(\mu \mathrm{eq} / \mathrm{L})}{\mathrm{Ca}}$ & $\underset{(\mu \mathrm{eq} / \mathrm{L})}{\mathrm{Mg}}$ & $\underset{(\mu \mathrm{eq} / \mathrm{L})}{\mathrm{Na}}$ & 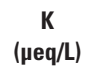 & $\begin{array}{c}\mathrm{NH}_{4} \\
(\mu \mathrm{eq} / \mathrm{L})\end{array}$ & $\underset{(\mu \mathrm{eq} / \mathrm{L})}{\mathrm{Cl}}$ & $\begin{array}{c}\mathrm{SO}_{4} \\
(\mu \mathrm{eq} / \mathrm{L})\end{array}$ & $\begin{array}{c}\mathrm{NO}_{3} \\
(\mu \mathrm{eq} / \mathrm{L})\end{array}$ & $\begin{array}{c}\text { DOC } \\
\text { (mg/L) }\end{array}$ & $\underset{(n g / L)}{\mathrm{Hg}}$ \\
\hline \multicolumn{17}{|c|}{1999 (cont.) } \\
\hline Laboratory Blank & 6/15/1999 & LB & -2.8 & 1.0 & 5.49 & 3.2 & $<3.1$ & $<1.7$ & $<1.0$ & 0.5 & $<1.0$ & $<1.0$ & $<0.6$ & 0.7 & $<0.4$ & na \\
\hline Laboratory Blank & 6/16/1999 & LB & -5.4 & 1.3 & 5.31 & 4.9 & $<3.1$ & $<1.7$ & $<1.0$ & $<0.4$ & $<1.0$ & $<1.0$ & $<0.6$ & $<0.4$ & $<0.4$ & na \\
\hline Laboratory Blank & 6/16/1999 & LB & -4.3 & 1.0 & 5.43 & 3.7 & $<3.1$ & $<1.7$ & $<1.0$ & $<0.4$ & $<1.0$ & $<1.0$ & $<0.6$ & $<0.4$ & $<0.4$ & na \\
\hline \multicolumn{17}{|c|}{2000} \\
\hline Apgar Lookout, Mont. & $3 / 6 / 2000$ & FB & 1.2 & 1.4 & 5.49 & 3.2 & $<3.1$ & $<1.7$ & $<1.0$ & $<0.4$ & $<1.0$ & $<1.0$ & $<0.6$ & $<0.4$ & 0.4 & na \\
\hline Culebra, Colo. & $2 / 27 / 2000$ & FB & 0.3 & 1.7 & 5.60 & 2.5 & 5.4 & $<1.7$ & $<1.0$ & $<0.4$ & $<1.0$ & $<1.0$ & 1.0 & 0.6 & $<0.4$ & na \\
\hline E2 (Glacier NP), Mont. & $3 / 20 / 2000$ & FB & 0.2 & 1.2 & 5.50 & 3.2 & $<3.1$ & $<1.7$ & $<1.0$ & $<0.4$ & $<1.0$ & $<1.0$ & $<0.6$ & $<0.4$ & na & na \\
\hline Grand Mesa, Colo. & $3 / 22 / 2000$ & FB & -3.8 & 1.3 & 5.07 & 8.5 & $<3.1$ & $<1.7$ & $<1.0$ & $<0.4$ & $<1.0$ & $<1.0$ & $<0.6$ & $<0.4$ & 0.4 & na \\
\hline Hopewell, N. Mex. & $3 / 12 / 2000$ & FB & -2.6 & 1.7 & 5.12 & 7.6 & $<3.1$ & $<1.7$ & $<1.0$ & $<0.4$ & $<1.0$ & $<1.0$ & $<0.6$ & $<0.4$ & 1.1 & na \\
\hline Independence Pass, Colo. & $4 / 10 / 2000$ & FB & 0.0 & 1.4 & 5.10 & 7.9 & $<3.1$ & $<1.7$ & $<1.0$ & $<0.4$ & $<1.0$ & $<1.0$ & $<0.6$ & $<0.4$ & 0.5 & na \\
\hline Lizard Head Pass, Colo. & $2 / 27 / 2000$ & FB & 1.1 & 2.0 & 5.73 & 1.9 & $<3.1$ & $<1.7$ & $<1.0$ & $<0.4$ & $<1.0$ & $<1.0$ & 1.0 & 0.6 & $<0.4$ & na \\
\hline Mount Belmont, Mont. & $3 / 3 / 2000$ & FB & -0.8 & 1.2 & 5.26 & 5.5 & $<3.1$ & $<1.7$ & $<1.0$ & $<0.4$ & $<1.0$ & $<1.0$ & $<0.6$ & $<0.4$ & $<0.4$ & na \\
\hline Phantom Valley, Colo. & $3 / 19 / 2000$ & FB & -2.7 & 1.3 & 5.34 & 4.6 & $<3.1$ & $<1.7$ & $<1.0$ & $<0.4$ & $<1.0$ & $<1.0$ & $<0.6$ & $<0.4$ & 0.7 & na \\
\hline Slumgullion Pass, Colo. & $3 / 25 / 2000$ & FB & -0.9 & 1.9 & 5.11 & 7.8 & $<3.1$ & $<1.7$ & $<1.0$ & $<0.4$ & $<1.0$ & $<1.0$ & $<0.6$ & 0.5 & 0.5 & na \\
\hline W5 (Glacier NP), Mont. & $3 / 21 / 2000$ & FB & 2.3 & 1.5 & 5.64 & 2.3 & $<3.1$ & $<1.7$ & 2.8 & 1.5 & $<1.0$ & 2.3 & $<0.6$ & $<0.4$ & 0.7 & na \\
\hline West Yellowstone, Mont. & $3 / 5 / 2000$ & FB & -0.1 & 1.7 & 5.18 & 6.6 & $<3.1$ & $<1.7$ & $<1.0$ & $<0.4$ & $<1.0$ & $<1.0$ & $<0.6$ & 0.5 & na & na \\
\hline Laboratory Blank & $2 / 29 / 2000$ & LB & na & 2.0 & na & na & $<3.1$ & $<1.7$ & $<1.0$ & $<0.4$ & $<1.0$ & $<1.0$ & 1.0 & 0.6 & $<0.4$ & na \\
\hline Laboratory Blank & $2 / 29 / 2000$ & LB & na & 2.2 & na & na & $<3.1$ & $<1.7$ & $<1.0$ & $<0.4$ & $<1.0$ & $<1.0$ & 1.0 & 0.7 & $<0.4$ & na \\
\hline Laboratory Blank & $5 / 1 / 2000$ & LB & -1.1 & 1.0 & 5.39 & 4.1 & $<3.1$ & $<1.7$ & $<1.0$ & $<0.4$ & $<1.0$ & $<1.0$ & $<0.6$ & $<0.4$ & $<0.4$ & na \\
\hline Laboratory Blank & $5 / 2 / 2000$ & LB & -4.2 & $<1.0$ & 5.38 & 4.2 & $<3.1$ & $<1.7$ & $<1.0$ & $<0.4$ & $<1.0$ & $<1.0$ & $<0.6$ & $<0.4$ & $<0.4$ & na \\
\hline \multicolumn{17}{|c|}{2001} \\
\hline Bowman 2 (Glacier NP), Mont. & $3 / 9 / 2001$ & FB & -2.3 & 1.4 & 5.53 & 3.0 & $<3.1$ & $<1.7$ & $<1.0$ & $<0.4$ & $<1.0$ & $<1.0$ & $<0.6$ & $<0.4$ & $<0.4$ & na \\
\hline $\begin{array}{l}\text { Dinah Shore } 1 \text { (Glacier NP), } \\
\text { Mont. }\end{array}$ & 3/9/2001 & FB & -0.9 & 1.5 & 5.58 & 2.6 & $<3.1$ & $<1.7$ & $<1.0$ & $<0.4$ & $<1.0$ & $<1.0$ & $<0.6$ & $<0.4$ & $<0.4$ & na \\
\hline Four Mile Meadow, Wyo. & $3 / 12 / 2001$ & FB & -3.0 & 1.5 & 5.36 & 4.4 & $<3.1$ & $<1.7$ & $<1.0$ & $<0.4$ & $<1.0$ & $<1.0$ & $<0.6$ & $<0.4$ & 0.4 & na \\
\hline Hopewell, N. Mex. & $3 / 16 / 2001$ & FB & -4.1 & 1.7 & 5.46 & 3.5 & $<3.1$ & $<1.7$ & $<1.0$ & $<0.4$ & $<1.0$ & $<1.0$ & $<0.6$ & $<0.4$ & $<0.4$ & na \\
\hline Mount Belmont, Mont. & $3 / 8 / 2001$ & FB & -0.2 & 1.7 & 5.60 & 2.5 & $<3.1$ & $<1.7$ & $<1.0$ & $<0.4$ & $<1.0$ & $<1.0$ & $<0.6$ & $<0.4$ & $<0.4$ & na \\
\hline Noisy Basin, Mont. & $3 / 8 / 2001$ & FB & -1.0 & 1.7 & 5.42 & 3.8 & $<3.1$ & $<1.7$ & $<1.0$ & $<0.4$ & $<1.0$ & $<1.0$ & $<0.6$ & $<0.4$ & $<0.4$ & na \\
\hline Slumgullion Pass, Colo. & $3 / 27 / 2001$ & FB & -2.4 & 1.4 & 5.29 & 5.1 & $<3.1$ & $<1.7$ & $<1.0$ & $<0.4$ & $<1.0$ & $<1.0$ & $<0.6$ & $<0.4$ & $<0.4$ & na \\
\hline W5 (Glacier NP), Mont. & 3/9/2001 & FB & -3.4 & 2.2 & 5.43 & 3.7 & $<3.1$ & $<1.7$ & $<1.0$ & $<0.4$ & $<1.0$ & 1.0 & $<0.6$ & 0.8 & $<0.4$ & na \\
\hline Laboratory Blank & 6/7/2001 & LB & -2.7 & 1.2 & 5.56 & 2.8 & $<3.1$ & $<1.7$ & $<1.0$ & $<0.4$ & $<1.0$ & $<1.0$ & $<0.6$ & $<0.4$ & $<0.4$ & na \\
\hline Laboratory Blank & $6 / 7 / 2001$ & LB & -3.4 & 1.3 & 5.55 & 2.8 & $<3.1$ & $<1.7$ & $<1.0$ & $<0.4$ & $<1.0$ & $<1.0$ & $<0.6$ & $<0.4$ & $<0.4$ & na \\
\hline
\end{tabular}


["FB" field blank; "LB" laboratory blank; ANC, acid neutralization capacity; $\mu$ eq/L, microequivalents per liter; SC, specific conductance at $25^{\circ} \mathrm{C}$; $\mu \mathrm{S} / \mathrm{cm}$, microsiemens per centimeter; $\mathrm{pH}$ values in standard units; $\mathrm{H}$, hydrogen; $\mathrm{Ca}$, calcium; $\mathrm{Mg}$, magnesium; $\mathrm{Na}$, sodium; $\mathrm{K}$, potassium; $\mathrm{NH}_{4}$, ammonium; $\mathrm{Cl}$, chloride; $\mathrm{SO}$, sulfate; $\mathrm{NO}$, nitrate; $\mathrm{DOC}$, dissolved organic carbon; mg/L, milligrams per liter; NADP, National Atmospheric Deposition Program; Hg, mercury; ng/L, nanograms per liter; na, not analyzed; <, below reporting limits; NP, National Park]

\begin{tabular}{|c|c|c|c|c|c|c|c|c|c|c|c|c|c|c|c|c|}
\hline Site name & $\begin{array}{l}\text { Sample } \\
\text { date }\end{array}$ & $\begin{array}{c}\text { Sample } \\
\text { type }\end{array}$ & $\begin{array}{c}\text { ANC } \\
\text { ( } \mu \mathrm{eq} / \mathrm{L})\end{array}$ & $\underset{(\mu \mathrm{S} / \mathrm{cm})}{\mathrm{SC}}$ & $\mathrm{pH}$ & $\underset{\text { ( } \mu \mathrm{eq} / \mathrm{L})}{\mathrm{H}}$ & $\begin{array}{c}\mathrm{Ca} \\
(\mu \mathrm{eq} / \mathrm{L})\end{array}$ & $\underset{(\mu \mathrm{eq} / \mathrm{L})}{\mathrm{Mg}}$ & $\underset{(\mu \mathrm{Na} / \mathrm{L})}{\mathrm{Na}}$ & 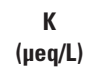 & $\begin{array}{c}\mathrm{NH}_{4} \\
(\mu \mathrm{eq} / \mathrm{L})\end{array}$ & $\underset{\text { (peq/L) }}{C I}$ & 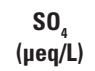 & $\begin{array}{c}\mathrm{NO}_{3} \\
(\mu \mathrm{eq} / \mathrm{L})\end{array}$ & $\begin{array}{c}\text { DOC } \\
\text { (mg/L) }\end{array}$ & $\underset{\text { (ng/L) }}{\mathrm{Hg}}$ \\
\hline \multicolumn{17}{|c|}{2001 (cont.) } \\
\hline Laboratory Blank & 6/6/2001 & LB & -4.3 & 2.1 & 5.31 & 4.9 & $<3.1$ & $<1.7$ & $<1.0$ & $<0.4$ & $<1.0$ & $<1.0$ & $<0.6$ & $<0.4$ & $<0.4$ & na \\
\hline Laboratory Blank & 6/5/2001 & LB & -5.0 & 2.3 & 5.28 & 5.2 & $<3.1$ & $<1.7$ & $<1.0$ & $<0.4$ & $<1.0$ & $<1.0$ & $<0.6$ & $<0.4$ & $<0.4$ & na \\
\hline Laboratory Blank & 6/7/2001 & LB & -5.2 & 3.1 & 5.56 & 2.8 & $<3.1$ & $<1.7$ & $<1.0$ & $<0.4$ & $<1.0$ & $<1.0$ & $<0.6$ & $<0.4$ & $<0.4$ & na \\
\hline Laboratory Blank & 6/4/2001 & LB & -8.1 & 3.3 & 5.15 & 7.1 & $<3.1$ & $<1.7$ & $<1.0$ & $<0.4$ & $<1.0$ & $<1.0$ & $<0.6$ & $<0.4$ & $<0.4$ & na \\
\hline Laboratory Blank & 6/7/2001 & LB & -8.4 & 2.3 & 5.31 & 4.9 & $<3.1$ & $<1.7$ & $<1.0$ & $<0.4$ & $<1.0$ & $<1.0$ & $<0.6$ & $<0.4$ & 0.4 & na \\
\hline \multicolumn{17}{|c|}{2002} \\
\hline Elk River, Colo. & $3 / 12 / 2002$ & FB & -3.2 & 4.0 & 5.53 & 3.0 & $<3.1$ & $<1.7$ & $<1.0$ & $<0.4$ & $<1.0$ & $<1.0$ & $<0.6$ & $<0.4$ & $<0.4$ & $<0.4$ \\
\hline Hopewell, N. Mex. & $3 / 15 / 2002$ & FB & -2.9 & 1.9 & 5.57 & 2.7 & $<3.1$ & $<1.7$ & $<1.0$ & $<0.4$ & $<1.0$ & $<1.0$ & $<0.6$ & $<0.4$ & $<0.4$ & $<0.4$ \\
\hline Kings Hill, Mont. & $3 / 2 / 2002$ & FB & -3.3 & 3.9 & 5.54 & 2.9 & $<3.1$ & $<1.7$ & $<1.0$ & $<0.4$ & $<1.0$ & $<1.0$ & $<0.6$ & $<0.4$ & $<0.4$ & $<0.4$ \\
\hline Loveland Pass, Colo. & 4/6/2002 & FB & -3.5 & 2.1 & 5.57 & 2.7 & $<3.1$ & $<1.7$ & $<1.0$ & $<0.4$ & $<1.0$ & $<1.0$ & $<0.6$ & $<0.4$ & $<0.4$ & 1.2 \\
\hline Phantom Valley, Colo. & $3 / 7 / 2002$ & FB & -3.6 & 3.2 & 5.55 & 2.8 & $<3.1$ & $<1.7$ & $<1.0$ & $<0.4$ & $<1.0$ & $<1.0$ & $<0.6$ & $<0.4$ & $<0.4$ & $<0.4$ \\
\hline Rabbit Ears 2, Colo. & $3 / 23 / 2002$ & FB & -4.4 & 2.8 & 5.48 & 3.3 & $<3.1$ & $<1.7$ & $<1.0$ & $<0.4$ & $<1.0$ & $<1.0$ & $<0.6$ & $<0.4$ & $<0.4$ & 0.7 \\
\hline Red Mountain Pass, Colo. & $3 / 27 / 2002$ & FB & -3.8 & 3.7 & 5.48 & 3.3 & $<3.1$ & $<1.7$ & $<1.0$ & $<0.4$ & $<1.0$ & $<1.0$ & $<0.6$ & $<0.4$ & $<0.4$ & $<0.4$ \\
\hline Laboratory Blank & $5 / 3 / 2002$ & LB & -4.6 & 1.1 & 5.51 & 3.1 & $<3.1$ & $<1.7$ & $<1.0$ & $<0.4$ & $<1.0$ & $<1.0$ & $<0.6$ & $<0.4$ & $<0.4$ & $<0.4$ \\
\hline Laboratory Blank & $6 / 5 / 2002$ & LB & -5.4 & 1.3 & 5.46 & 3.5 & $<3.1$ & $<1.7$ & $<1.0$ & $<0.4$ & $<1.0$ & $<1.0$ & $<0.6$ & $<0.4$ & $<0.4$ & $<0.4$ \\
\hline Laboratory Blank & $6 / 5 / 2002$ & LB & -7.5 & 1.2 & 5.43 & 3.7 & $<3.1$ & $<1.7$ & $<1.0$ & $<0.4$ & $<1.0$ & $<1.0$ & $<0.6$ & $<0.4$ & $<0.4$ & $<0.4$ \\
\hline Laboratory Blank & $3 / 21 / 2002$ & LB & -4.0 & 1.4 & 5.45 & 3.5 & $<3.1$ & $<1.7$ & $<1.0$ & $<0.4$ & $<1.0$ & $<1.0$ & $<0.6$ & $<0.4$ & $<0.4$ & 0.7 \\
\hline Laboratory Blank & 6/6/2002 & LB & -6.8 & 1.0 & 5.46 & 3.5 & $<3.1$ & $<1.7$ & $<1.0$ & $<0.4$ & $<1.0$ & $<1.0$ & $<0.6$ & $<0.4$ & $<0.4$ & 0.7 \\
\hline \multicolumn{17}{|c|}{2003} \\
\hline Arapaho Creek, Colo. & $3 / 25 / 2003$ & FB & -2.6 & 1.3 & 5.51 & 3.1 & $<3.1$ & $<1.7$ & $<1.0$ & $<0.4$ & $<1.0$ & $<1.0$ & $<0.6$ & $<0.4$ & $<0.4$ & $<0.4$ \\
\hline Elkhart Park, Wyo. & $3 / 25 / 2003$ & FB & -2.9 & 1.7 & 5.39 & 4.1 & $<3.1$ & $<1.7$ & $<1.0$ & $<0.4$ & $<1.0$ & $<1.0$ & $<0.6$ & $<0.4$ & $<0.4$ & 0.4 \\
\hline Grand Mesa, Colo. & $3 / 24 / 2003$ & $\mathrm{FB}$ & -4.5 & 3.7 & 5.21 & 6.2 & $<3.1$ & $<1.7$ & $<1.0$ & $<0.4$ & $<1.0$ & $<1.0$ & $<0.6$ & $<0.4$ & $<0.4$ & $<0.4$ \\
\hline Gypsum Creek, Wyo. & $3 / 26 / 2003$ & FB & -2.9 & 1.6 & 5.49 & 3.2 & $<3.1$ & $<1.7$ & $<1.0$ & $<0.4$ & $<1.0$ & $<1.0$ & $<0.6$ & $<0.4$ & $<0.4$ & $<0.4$ \\
\hline Hopewell, N. Mex. & $3 / 28 / 2003$ & FB & -2.2 & 2.2 & 5.47 & 3.4 & $<3.1$ & $<1.7$ & $<1.0$ & $<0.4$ & $<1.0$ & $<1.0$ & $<0.6$ & $<0.4$ & $<0.4$ & $<0.4$ \\
\hline Monida Pass, Mont. & $2 / 27 / 2003$ & FB & -0.8 & 1.3 & 5.64 & 2.3 & $<3.1$ & $<1.7$ & $<1.0$ & $<0.4$ & $<1.0$ & $<1.0$ & $<0.6$ & $<0.4$ & $<0.4$ & 0.8 \\
\hline Phantom Valley, Colo. & $3 / 27 / 2003$ & FB & -3.8 & 2.0 & 5.35 & 4.5 & $<3.1$ & $<1.7$ & $<1.0$ & $<0.4$ & $<1.0$ & $<1.0$ & $<0.6$ & $<0.4$ & $<0.4$ & $<0.4$ \\
\hline Red Mountain Pass, Colo. & $3 / 29 / 2003$ & FB & -3.8 & 1.5 & 5.51 & 3.1 & $<3.1$ & $<1.7$ & $<1.0$ & $<0.4$ & $<1.0$ & $<1.0$ & $<0.6$ & $<0.4$ & $<0.4$ & 0.4 \\
\hline Laboratory Blank & $5 / 20 / 2003$ & LB & -3.9 & 1.4 & 5.56 & 2.8 & $<3.1$ & $<1.7$ & $<1.0$ & $<0.4$ & $<1.0$ & $<1.0$ & $<0.6$ & $<0.4$ & $<0.4$ & 1.4 \\
\hline Laboratory Blank & $5 / 21 / 2003$ & LB & -4.9 & 1.2 & 5.59 & 2.6 & $<3.1$ & $<1.7$ & $<1.0$ & $<0.4$ & $<1.0$ & $<1.0$ & $<0.6$ & $<0.4$ & $<0.4$ & 1.0 \\
\hline Laboratory Blank & $5 / 22 / 2003$ & LB & -3.8 & 1.5 & 5.52 & 3.0 & $<3.1$ & $<1.7$ & $<1.0$ & $<0.4$ & $<1.0$ & $<1.0$ & $<0.6$ & $<0.4$ & $<0.4$ & 1.0 \\
\hline Laboratory Blank & $5 / 22 / 2003$ & LB & -4.4 & 1.3 & 5.51 & 3.1 & $<3.1$ & $<1.7$ & $<1.0$ & $<0.4$ & $<1.0$ & $<1.0$ & $<0.6$ & $<0.4$ & $<0.4$ & 1.3 \\
\hline
\end{tabular}


Table 18. Quality-assurance data: selected chemical concentrations in blank samples for 1993-2008.—Continued

["FB" field blank; "LB" laboratory blank; ANC, acid neutralization capacity; $\mu$ eq/L, microequivalents per liter; $\mathrm{SC}$, specific conductance at $25^{\circ} \mathrm{C}$; $\mu \mathrm{S} / \mathrm{cm}, \mathrm{microsiemens}$ per centimeter; $\mathrm{pH}$ values in standard units; $\mathrm{H}$, hydrogen; $\mathrm{Ca}$, calcium; $\mathrm{Mg}$, magnesium; $\mathrm{Na}$, sodium; $\mathrm{K}$, potassium; $\mathrm{NH}_{4}$, ammonium; $\mathrm{Cl}$, chloride; $\mathrm{SO}_{4}$, sulfate; $\mathrm{NO}_{3}$, nitrate; $\mathrm{DOC}$, dissolved organic carbon; $\mathrm{mg} / \mathrm{L}$, milligrams per liter; NADP, National Atmospheric Deposition Program; Hg, mercury; ng/L, nanograms per liter; na, not analyzed; <, below reporting limits; NP, National Park]

\begin{tabular}{|c|c|c|c|c|c|c|c|c|c|c|c|c|c|c|c|c|}
\hline Site name & $\begin{array}{c}\text { Sample } \\
\text { date }\end{array}$ & $\begin{array}{c}\text { Sample } \\
\text { type }\end{array}$ & $\begin{array}{c}\text { ANC } \\
\text { ( } \mu \text { eq/L) }\end{array}$ & $\begin{array}{c}\text { SC } \\
(\mu \mathrm{S} / \mathrm{cm})\end{array}$ & pH & 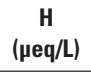 & $\begin{array}{c}\text { Ca } \\
(\mu \mathrm{eq} / \mathrm{L})\end{array}$ & $\underset{(\mu \mathrm{gg} / \mathrm{L})}{\mathrm{Mg}}$ & $\begin{array}{c}\mathrm{Na} \\
\text { ( } \mu \mathrm{eq} / \mathrm{L})\end{array}$ & $\begin{array}{c}K \\
(\mu \mathrm{eq} / \mathrm{L})\end{array}$ & $\begin{array}{c}\mathrm{NH}_{4} \\
\text { ( } \mu \mathrm{eq} / \mathrm{L} \text { ) }\end{array}$ & $\underset{(\mu \mathrm{eq} / \mathrm{L})}{\mathrm{Cl}}$ & $\begin{array}{c}\mathrm{SO}_{4} \\
(\mu \mathrm{eq} / \mathrm{L})\end{array}$ & 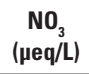 & $\begin{array}{c}\text { DOC } \\
\text { (mg/L) }\end{array}$ & $\begin{array}{c}\mathrm{Hg} \\
\text { (ng/L) }\end{array}$ \\
\hline \multicolumn{17}{|c|}{2004} \\
\hline Lake Irene Forest, Colo. & $3 / 30 / 2004$ & FB & -10.6 & 2.7 & 5.25 & 5.6 & $<3.1$ & $<1.7$ & $<1.0$ & $<0.4$ & $<1.0$ & $<1.0$ & $<0.6$ & 0.5 & $<0.4$ & 0.6 \\
\hline West Yellowstone, Mont. & $2 / 26 / 2004$ & FB & -4.2 & 1.9 & 5.38 & 4.2 & $<3.1$ & $<1.7$ & $<1.0$ & $<0.4$ & $<1.0$ & $<1.0$ & $<0.6$ & $<0.4$ & $<0.4$ & $<0.4$ \\
\hline Laboratory Blank & $5 / 26 / 2004$ & LB & -4.4 & 1.8 & 5.48 & 3.3 & $<3.1$ & $<1.7$ & $<1.0$ & $<0.4$ & $<1.0$ & $<1.0$ & $<0.6$ & $<0.4$ & $<0.4$ & $<0.4$ \\
\hline Laboratory Blank & $5 / 27 / 2004$ & LB & -2.5 & 1.4 & 5.74 & 1.8 & $<3.1$ & $<1.7$ & $<1.0$ & $<0.4$ & $<1.0$ & $<1.0$ & $<0.6$ & $<0.4$ & $<0.4$ & $<0.4$ \\
\hline \multicolumn{17}{|c|}{2005} \\
\hline Galena Summit, Idaho & $3 / 10 / 2005$ & FB & -6.8 & 3.9 & 5.20 & 6.3 & $<3.1$ & $<1.7$ & $<1.0$ & $<0.4$ & $<1.0$ & $<1.0$ & $<0.6$ & 0.4 & $<0.4$ & 0.9 \\
\hline Lake Irene Forest, Colo. & $4 / 5 / 2005$ & FB & -4.9 & 2.4 & 5.46 & 3.5 & $<3.1$ & $<1.7$ & $<1.0$ & $<0.4$ & $<1.0$ & $<1.0$ & $<0.6$ & $<0.4$ & $<0.4$ & na \\
\hline Loveland Pass, Colo. & 4/9/2005 & FB & -1.7 & 2.2 & 5.72 & 1.9 & $<3.1$ & $<1.7$ & $<1.0$ & $<0.4$ & $<1.0$ & $<1.0$ & $<0.6$ & $<0.4$ & $<0.4$ & $<0.4$ \\
\hline Laboratory Blank & $5 / 26 / 2005$ & LB & -4.0 & 1.4 & 5.71 & 1.9 & $<3.1$ & $<1.7$ & 4.0 & 2.4 & $<1.0$ & $<1.0$ & $<0.6$ & $<0.4$ & $<0.4$ & $<0.4$ \\
\hline Laboratory Blank & $5 / 26 / 2005$ & LB & -2.4 & 1.4 & 5.71 & 1.9 & $<3.1$ & $<1.7$ & $<1.0$ & $<0.4$ & $<1.0$ & $<1.0$ & $<0.6$ & $<0.4$ & $<0.4$ & 2.4 \\
\hline Laboratory Blank & $5 / 26 / 2005$ & LB & -0.1 & 1.9 & 5.58 & 2.6 & $<3.1$ & $<1.7$ & $<1.0$ & $<0.4$ & $<1.0$ & $<1.0$ & $<0.6$ & $<0.4$ & $<0.4$ & $<0.4$ \\
\hline \multicolumn{17}{|c|}{2006} \\
\hline Galena Summit, Idaho & 3/9/2006 & FB & -3.3 & 2.2 & 5.43 & 3.7 & na & $<1.7$ & $<1.0$ & $<0.4$ & $<1.0$ & $<1.0$ & $<0.6$ & $<0.4$ & $<0.4$ & $<0.4$ \\
\hline Mosca Pass, Colo. & $3 / 23 / 2006$ & FB & -2.4 & 2.3 & 5.41 & 3.9 & na & $<1.7$ & $<1.0$ & $<0.4$ & $<1.0$ & 1.1 & $<0.6$ & 0.4 & $<0.4$ & $<0.4$ \\
\hline Laboratory Blank & $5 / 19 / 2006$ & LB & -1.9 & 1.6 & 5.56 & 2.8 & na & $<1.7$ & $<1.0$ & $<0.4$ & $<1.0$ & $<1.0$ & $<0.6$ & $<0.4$ & 1.3 & $<0.4$ \\
\hline Laboratory Blank & $5 / 24 / 2006$ & LB & -2.0 & 1.8 & 5.48 & 3.3 & na & $<1.7$ & $<1.0$ & $<0.4$ & $<1.0$ & $<1.0$ & $<0.6$ & $<0.4$ & $<0.4$ & $<0.4$ \\
\hline Laboratory Blank & $5 / 25 / 2006$ & LB & -2.0 & 1.5 & 5.55 & 2.8 & na & $<1.7$ & $<1.0$ & $<0.4$ & $<1.0$ & $<1.0$ & $<0.6$ & $<0.4$ & $<0.4$ & na \\
\hline \multicolumn{17}{|c|}{2007} \\
\hline Elkhart Park, Wyo. & $3 / 14 / 2007$ & FB & -2.7 & 2.5 & 5.40 & 4.0 & $<3.1$ & $<1.7$ & $<1.0$ & $<0.4$ & $<1.0$ & $<1.0$ & $<0.6$ & $<0.4$ & $<0.4$ & $<0.4$ \\
\hline Galena Summit, Idaho & $3 / 8 / 2007$ & FB & 1.5 & 3.0 & 5.34 & 4.6 & $<3.1$ & $<1.7$ & $<1.0$ & $<0.4$ & $<1.0$ & $<1.0$ & $<0.6$ & $<0.4$ & $<0.4$ & 0.5 \\
\hline Taos Ski Valley, N. Mex. & $3 / 25 / 2007$ & FB & -0.8 & 2.4 & 5.56 & 2.8 & $<3.1$ & $<1.7$ & $<1.0$ & $<0.4$ & $<1.0$ & $<1.0$ & $<0.6$ & $<0.4$ & $<0.4$ & 0.5 \\
\hline Laboratory Blank & $5 / 15 / 2007$ & LB & -0.5 & 2.1 & 5.60 & 2.5 & $<3.1$ & $<1.7$ & $<1.0$ & $<0.4$ & $<1.0$ & $<1.0$ & $<0.6$ & $<0.4$ & $<0.4$ & $<0.4$ \\
\hline Laboratory Blank & $5 / 17 / 2007$ & LB & 0.0 & 2.1 & 5.59 & 2.6 & $<3.1$ & $<1.7$ & $<1.0$ & $<0.4$ & $<1.0$ & $<1.0$ & $<0.6$ & $<0.4$ & $<0.4$ & $<0.4$ \\
\hline Laboratory Blank & $5 / 18 / 2007$ & & 0.5 & 2.0 & 5.65 & 2.2 & 4.0 & $<1.7$ & $<1.0$ & $<0.4$ & $<1.0$ & $<1.0$ & $<0.6$ & $<0.4$ & $<0.4$ & $<0.4$ \\
\hline \multicolumn{17}{|c|}{2008} \\
\hline Elkhart Park, Wyo. & $3 / 18 / 2008$ & FB & -1.8 & 3.4 & 5.27 & 5.4 & $<3.1$ & $<1.7$ & $<1.0$ & $<0.4$ & $<1.0$ & $<1.0$ & $<0.6$ & 0.4 & $<0.4$ & $<0.4$ \\
\hline Galena Summit, Idaho & $3 / 6 / 2008$ & FB & -3.1 & 2.5 & 5.44 & 3.6 & $<3.1$ & $<1.7$ & $<1.0$ & $<0.4$ & $<1.0$ & $<1.0$ & $<0.6$ & $<0.4$ & $<0.4$ & $<0.4$ \\
\hline Laboratory Blank & $4 / 24 / 2008$ & LB & -2.5 & 1.3 & 5.69 & 2.0 & $<3.1$ & $<1.7$ & $<1.0$ & $<0.4$ & $<1.0$ & $<1.0$ & $<0.6$ & $<0.4$ & $<0.4$ & $<0.4$ \\
\hline Laboratory Blank & $4 / 28 / 2008$ & LB & -1.3 & 1.4 & 5.68 & 2.1 & $<3.1$ & $<1.7$ & $<1.0$ & $<0.4$ & $<1.0$ & $<1.0$ & $<0.6$ & $<0.4$ & $<0.4$ & $<0.4$ \\
\hline Laboratory Blank & $4 / 29 / 2008$ & LB & -1.9 & 1.5 & 5.62 & 2.4 & $<3.1$ & $<1.7$ & $<1.0$ & $<0.4$ & $<1.0$ & $<1.0$ & $<0.6$ & $<0.4$ & $<0.4$ & 0.7 \\
\hline
\end{tabular}

${ }^{1}$ High concentrations in this outlier laboratory blank are uncharacteristic of other blanks. 
Table 19. Quality-assurance data: relative percent differences in concentrations between environmental and replicate samples for 1993-2008.

[E, depth-integrated environmental; FR, field replicate; RPD, relative percent difference; $\mu$ eq/L, microequivalents per liter; $\mathrm{Ca}$, calcium; Mg, magnesium; Na, sodium; $\mathrm{K}$, potassium; $\mathrm{NH}_{4}$, ammonium; $\mathrm{Cl}$, chloride; $\mathrm{SO}_{4}$, sulfate; $\mathrm{NO}_{3}$, nitrate; DOC, dissolved organic carbon; mg/L, milligrams per liter; $\mathrm{NADP}$, National Atmospheric Deposition Program; Hg, mercury; ng/L, nanograms per liter; na, not analyzed; <, below reporting limits; NP, National Park]

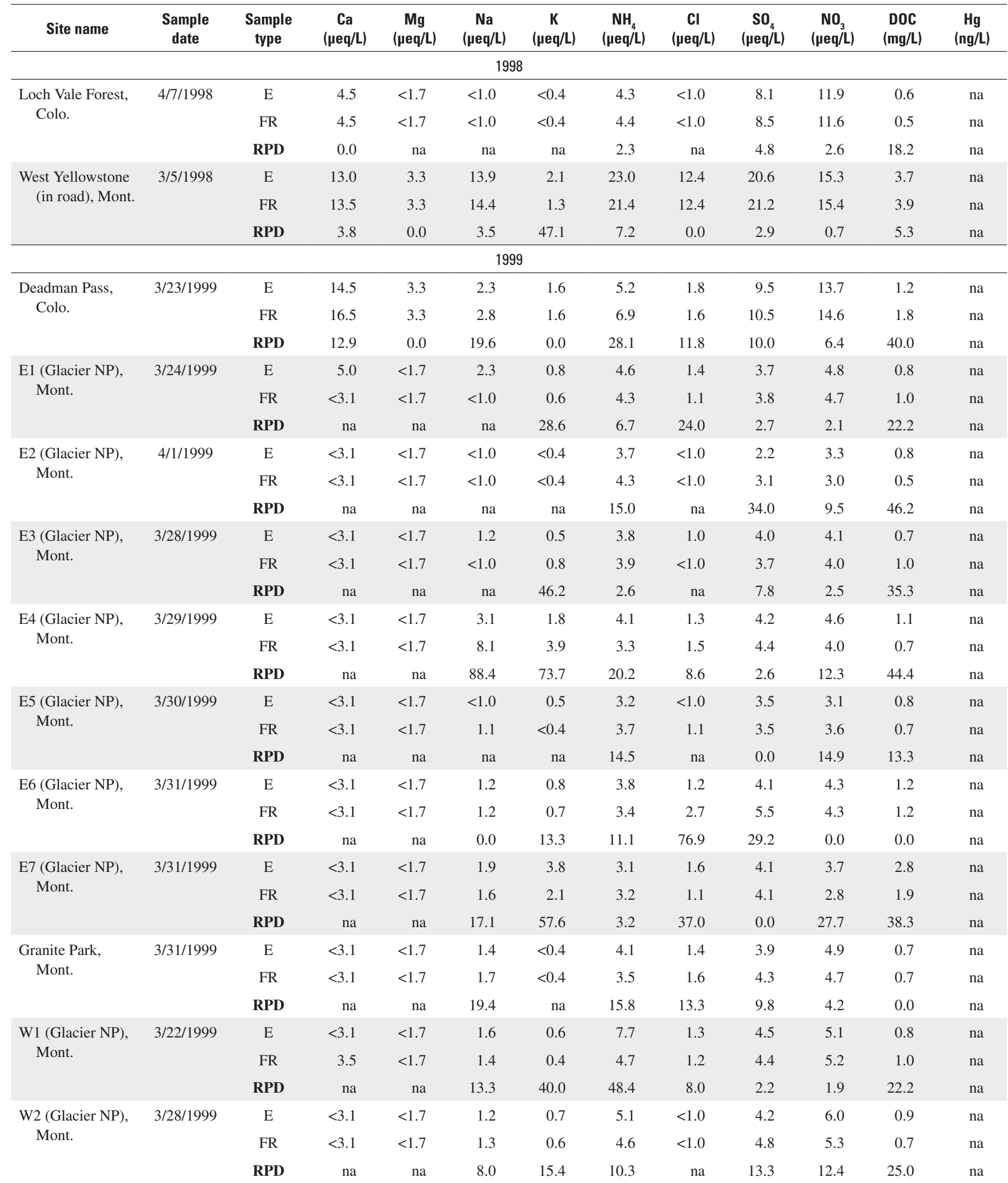


Table 19. Quality-assurance data: relative percent differences in concentrations between environmental and replicate samples for 1993-2008.-Continued

[E, depth-integrated environmental; FR, field replicate; RPD, relative percent difference; $\mu \mathrm{eq} / \mathrm{L}$, microequivalents per liter; $\mathrm{Ca}$, calcium; $\mathrm{Mg}, \mathrm{magnesium} ; \mathrm{Na}$, sodium; K, potassium; $\mathrm{NH}_{4}$, ammonium; $\mathrm{Cl}$, chloride; $\mathrm{SO}_{4}$, sulfate; $\mathrm{NO}_{3}$, nitrate; DOC, dissolved organic carbon; mg/L, milligrams per liter; NADP, National Atmospheric Deposition Program; Hg, mercury; ng/L, nanograms per liter; na, not analyzed; <, below reporting limits; NP, National Park]

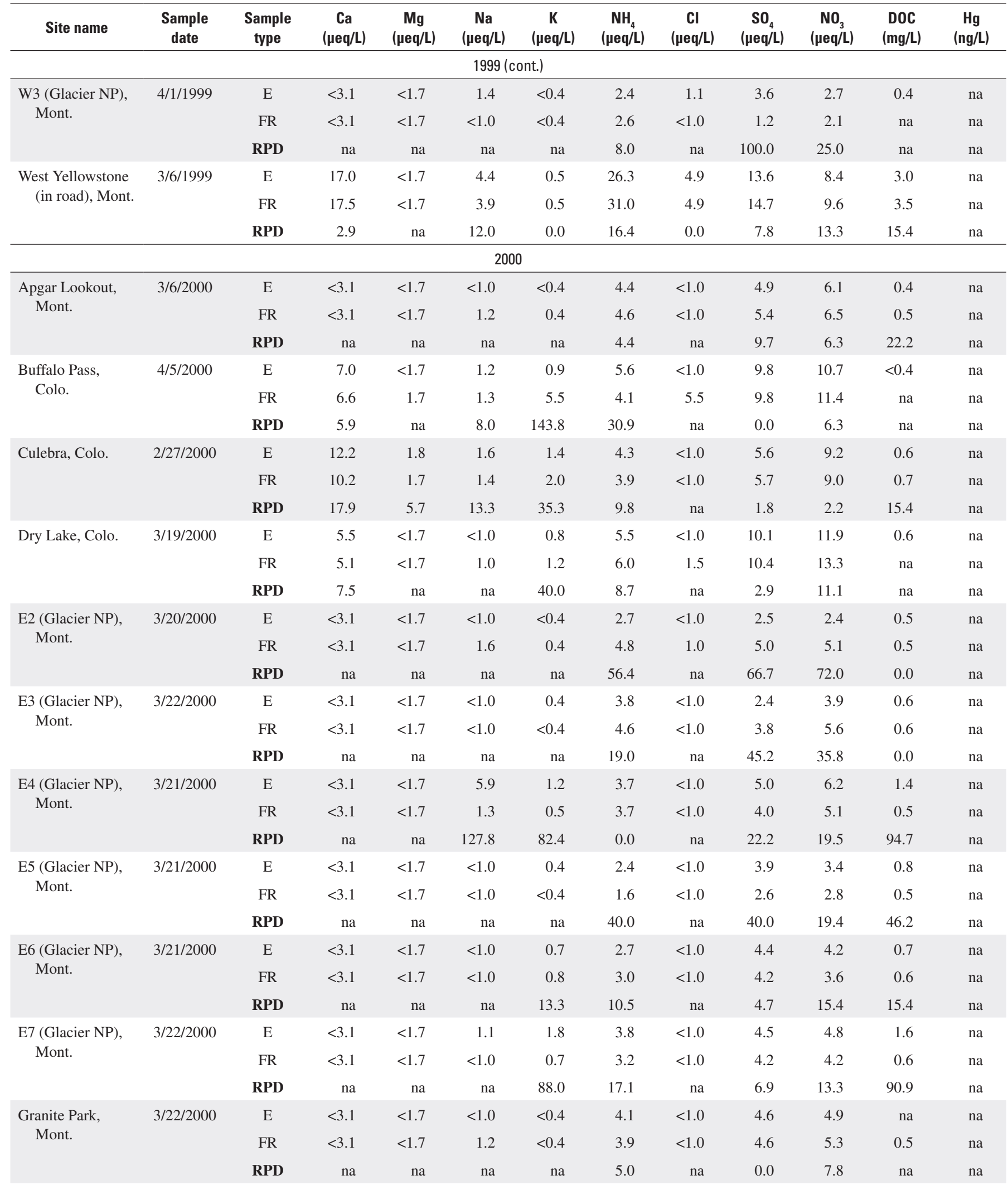


Table 19. Quality-assurance data: relative percent differences in concentrations between environmental and replicate samples for 1993-2008.-Continued

[E, depth-integrated environmental; FR, field replicate; RPD, relative percent difference; $\mu$ eq/L, microequivalents per liter; $\mathrm{Ca}$, calcium; Mg, magnesium; Na, sodium; K, potassium; $\mathrm{NH}_{4}$, ammonium; $\mathrm{Cl}$, chloride; $\mathrm{SO}_{4}$, sulfate; $\mathrm{NO}_{3}$, nitrate; DOC, dissolved organic carbon; mg/L, milligrams per liter; NADP, National Atmospheric Deposition Program; Hg, mercury; ng/L, nanograms per liter; na, not analyzed; <, below reporting limits; NP, National Park]

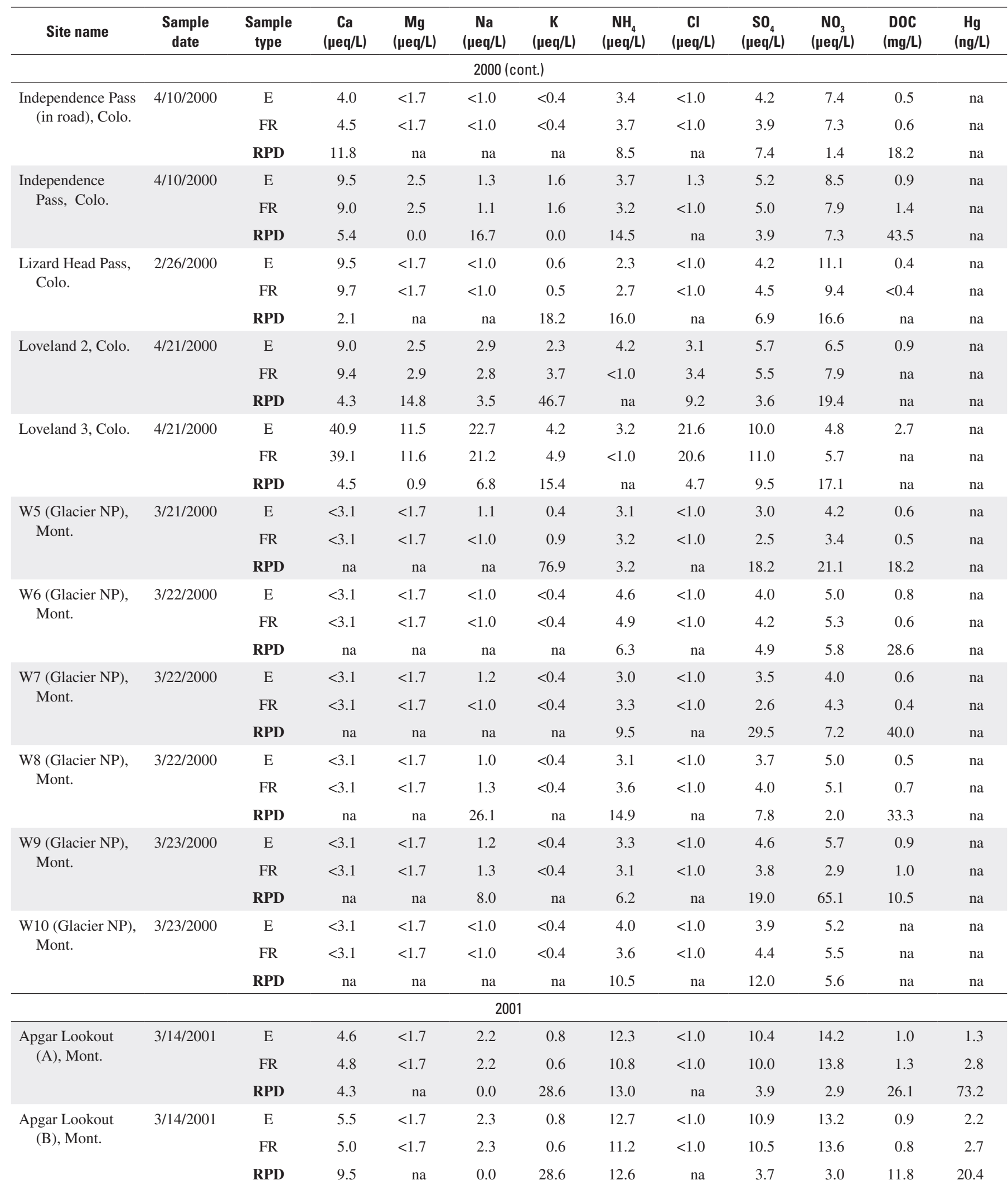


Table 19. Quality-assurance data: relative percent differences in concentrations between environmental and replicate samples for 1993-2008.-Continued

[E, depth-integrated environmental; FR, field replicate; RPD, relative percent difference; $\mu \mathrm{eq} / \mathrm{L}$, microequivalents per liter; $\mathrm{Ca}$, calcium; Mg, magnesium; Na, sodium; K, potassium; $\mathrm{NH}_{4}$, ammonium; $\mathrm{Cl}$, chloride; $\mathrm{SO}_{4}$, sulfate; $\mathrm{NO}_{3}$, nitrate; DOC, dissolved organic carbon; mg/L, milligrams per liter; NADP, National Atmospheric Deposition Program; Hg, mercury; ng/L, nanograms per liter; na, not analyzed; <, below reporting limits; NP, National Park]

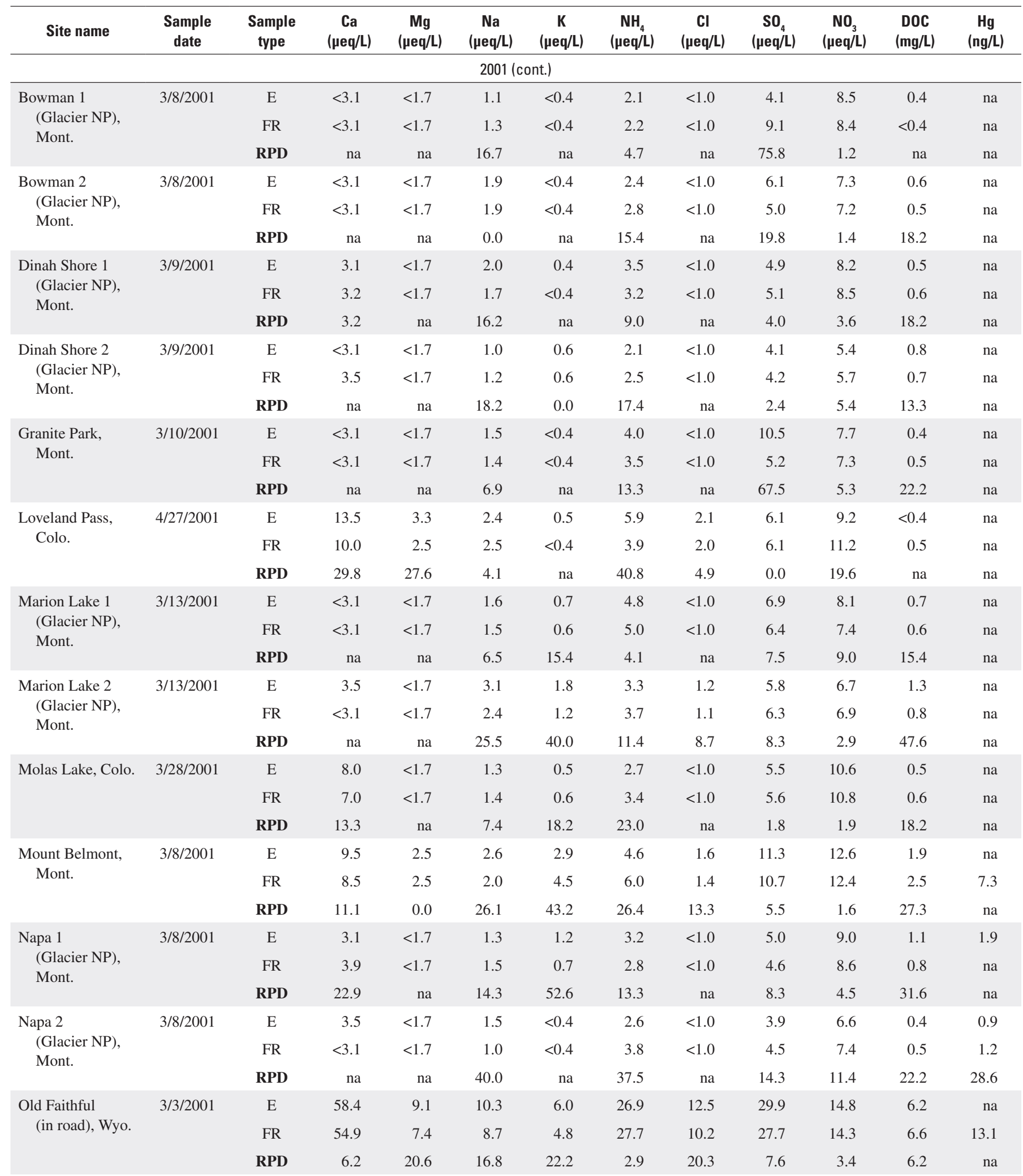


Table 19. Quality-assurance data: relative percent differences in concentrations between environmental and replicate samples for 1993-2008.-Continued

[E, depth-integrated environmental; FR, field replicate; RPD, relative percent difference; $\mu$ eq/L, microequivalents per liter; $\mathrm{Ca}$, calcium; Mg, magnesium; Na, sodium; K, potassium; $\mathrm{NH}_{4}$, ammonium; $\mathrm{Cl}$, chloride; $\mathrm{SO}_{4}$, sulfate; $\mathrm{NO}_{3}$, nitrate; DOC, dissolved organic carbon; mg/L, milligrams per liter; $\mathrm{NADP}$, National Atmospheric Deposition Program; Hg, mercury; ng/L, nanograms per liter; na, not analyzed; <, below reporting limits; NP, National Park]

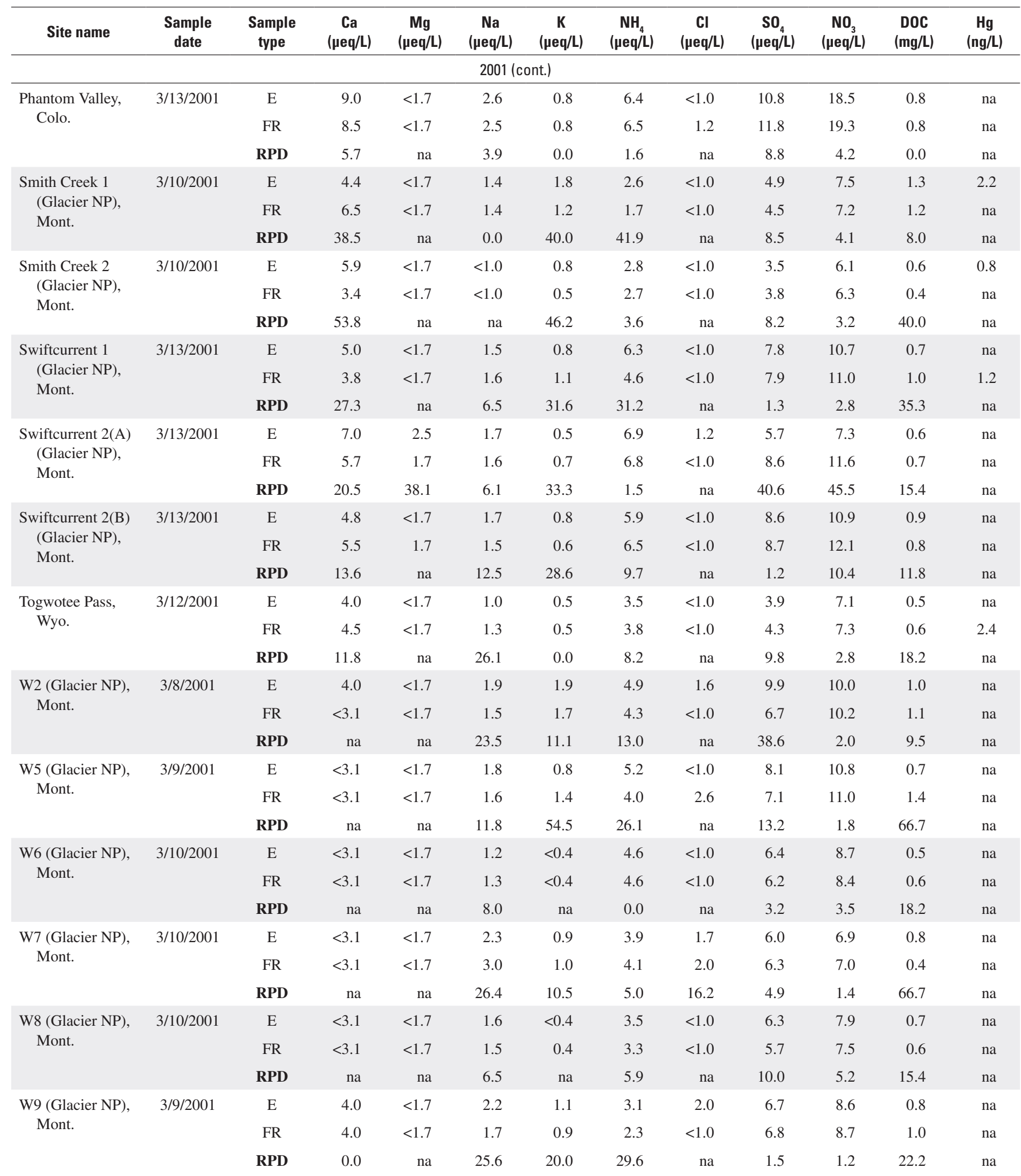


Table 19. Quality-assurance data: relative percent differences in concentrations between environmental and replicate samples for 1993-2008.-Continued

[E, depth-integrated environmental; FR, field replicate; RPD, relative percent difference; $\mu \mathrm{eq} / \mathrm{L}$, microequivalents per liter; $\mathrm{Ca}$, calcium; Mg, magnesium; Na, sodium; $\mathrm{K}$, potassium; $\mathrm{NH}_{4}$, ammonium; $\mathrm{Cl}$, chloride; $\mathrm{SO}_{4}$, sulfate; $\mathrm{NO}_{3}$, nitrate; DOC, dissolved organic carbon; mg/L, milligrams per liter; $\mathrm{NADP}$, National Atmospheric Deposition Program; Hg, mercury; ng/L, nanograms per liter; na, not analyzed; <, below reporting limits; NP, National Park]

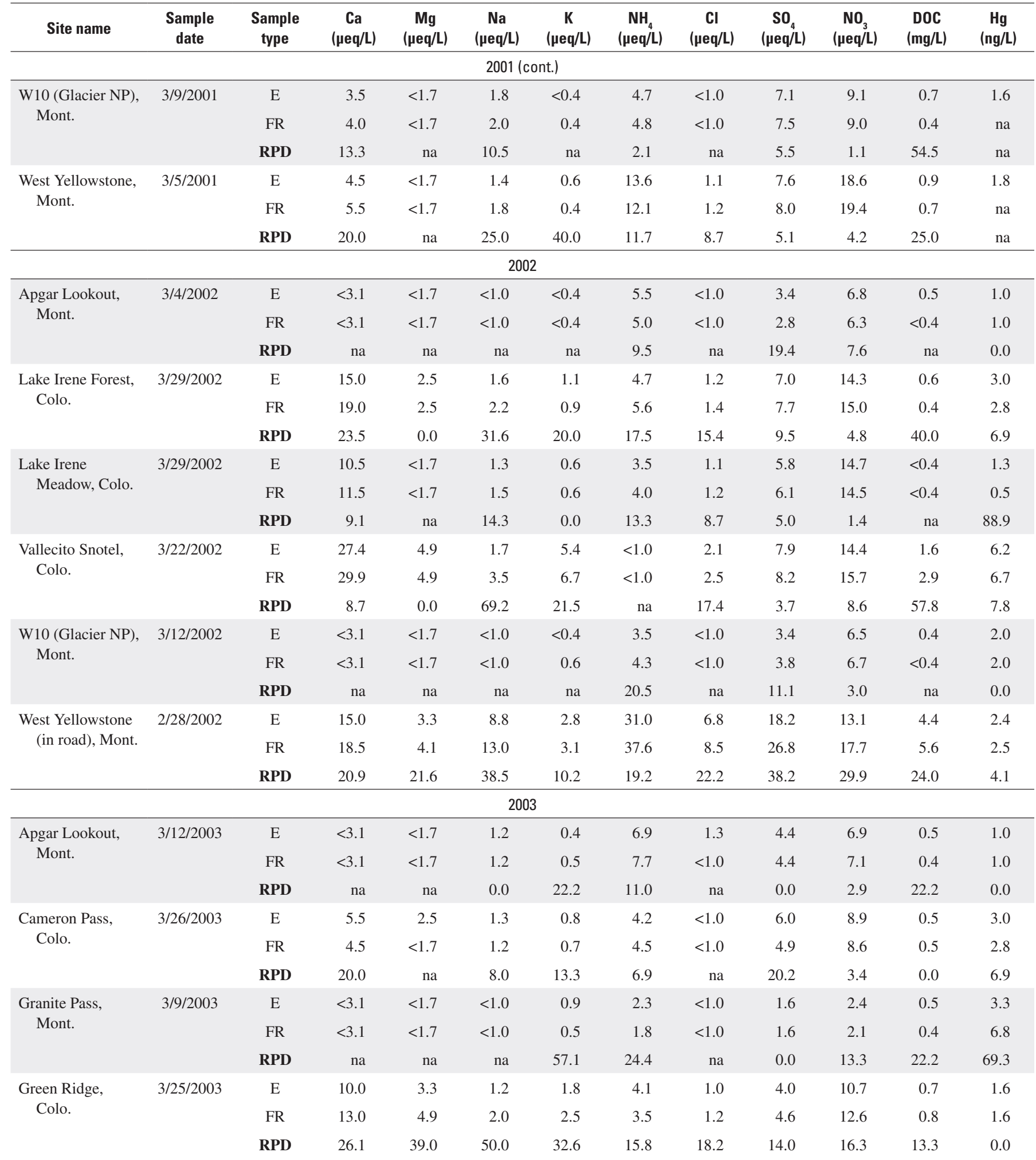


Table 19. Quality-assurance data: relative percent differences in concentrations between environmental and replicate samples for 1993-2008.-Continued

[E, depth-integrated environmental; FR, field replicate; RPD, relative percent difference; $\mu$ eq/L, microequivalents per liter; $\mathrm{Ca}$, calcium; Mg, magnesium; Na, sodium; $\mathrm{K}$, potassium; $\mathrm{NH}_{4}$, ammonium; $\mathrm{Cl}$, chloride; $\mathrm{SO}_{4}$, sulfate; $\mathrm{NO}_{3}$, nitrate; DOC, dissolved organic carbon; mg/L, milligrams per liter; $\mathrm{NADP}$, National Atmospheric Deposition Program; Hg, mercury; ng/L, nanograms per liter; na, not analyzed; <, below reporting limits; NP, National Park]

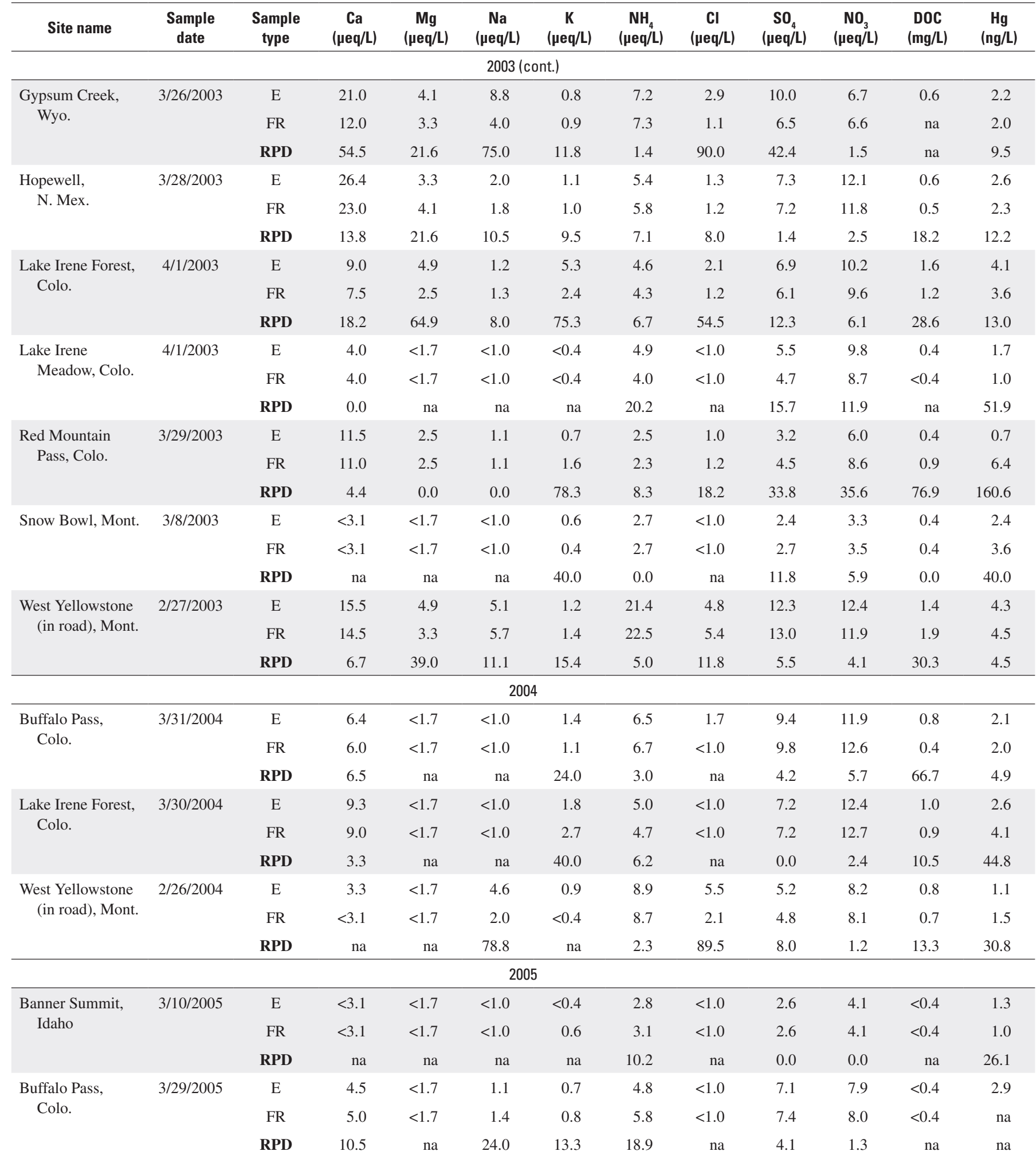


Table 19. Quality-assurance data: relative percent differences in concentrations between environmental and replicate samples for 1993-2008.-Continued

[E, depth-integrated environmental; FR, field replicate; RPD, relative percent difference; $\mu \mathrm{eq} / \mathrm{L}$, microequivalents per liter; $\mathrm{Ca}$, calcium; Mg, magnesium; Na, sodium; K, potassium; $\mathrm{NH}_{4}$, ammonium; $\mathrm{Cl}$, chloride; $\mathrm{SO}_{4}$, sulfate; $\mathrm{NO}_{3}$, nitrate; DOC, dissolved organic carbon; mg/L, milligrams per liter; NADP, National Atmospheric Deposition Program; Hg, mercury; ng/L, nanograms per liter; na, not analyzed; <, below reporting limits; NP, National Park]

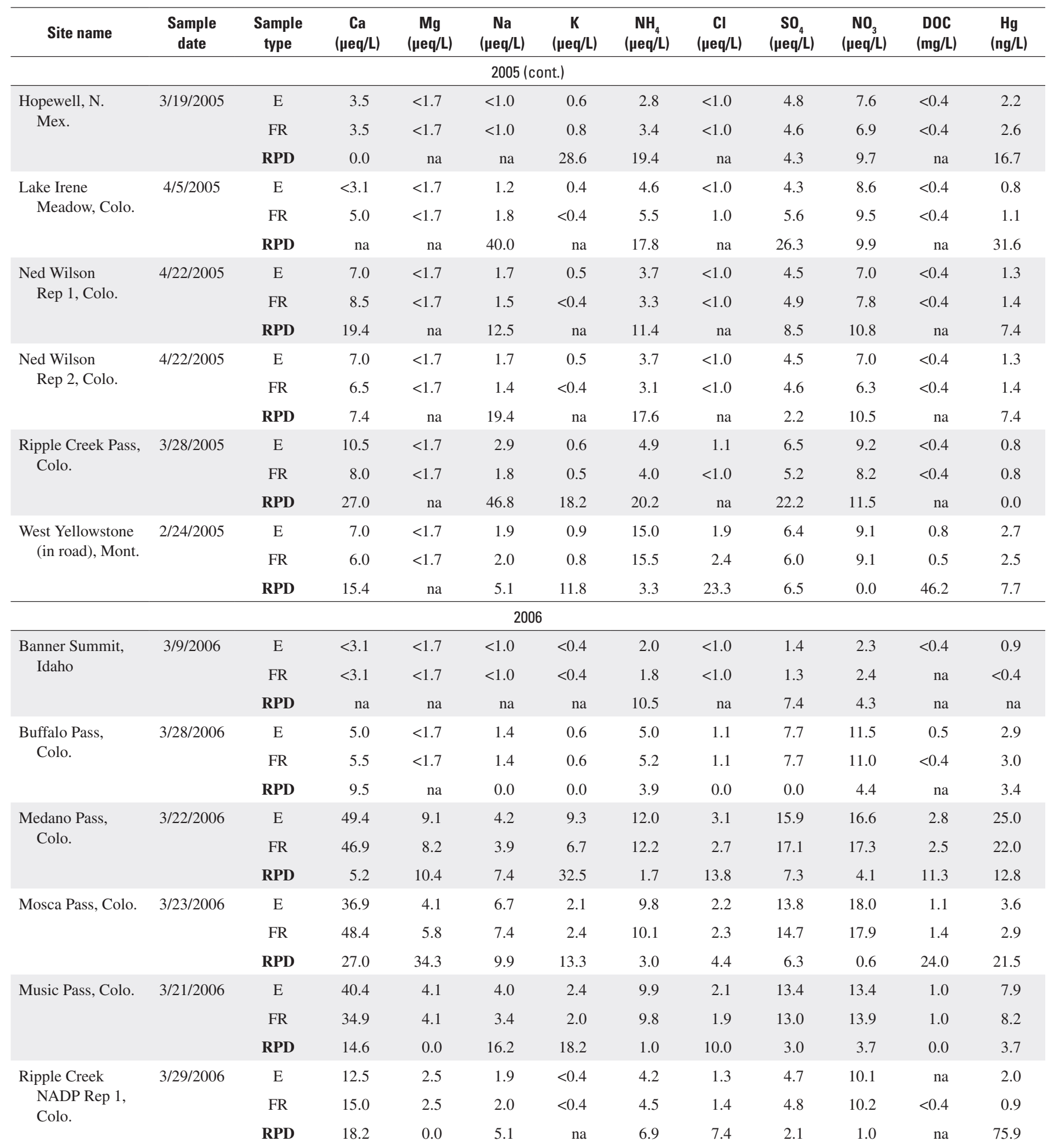


Table 19. Quality-assurance data: relative percent differences in concentrations between environmental and replicate samples for 1993-2008.-Continued

[E, depth-integrated environmental; FR, field replicate; RPD, relative percent difference; $\mu$ eq/L, microequivalents per liter; $\mathrm{Ca}$, calcium; Mg, magnesium; Na, sodium; K, potassium; $\mathrm{NH}_{4}$, ammonium; $\mathrm{Cl}$, chloride; $\mathrm{SO}_{4}$, sulfate; $\mathrm{NO}_{3}$, nitrate; DOC, dissolved organic carbon; mg/L, milligrams per liter; NADP, National Atmospheric Deposition Program; Hg, mercury; ng/L, nanograms per liter; na, not analyzed; <, below reporting limits; NP, National Park]

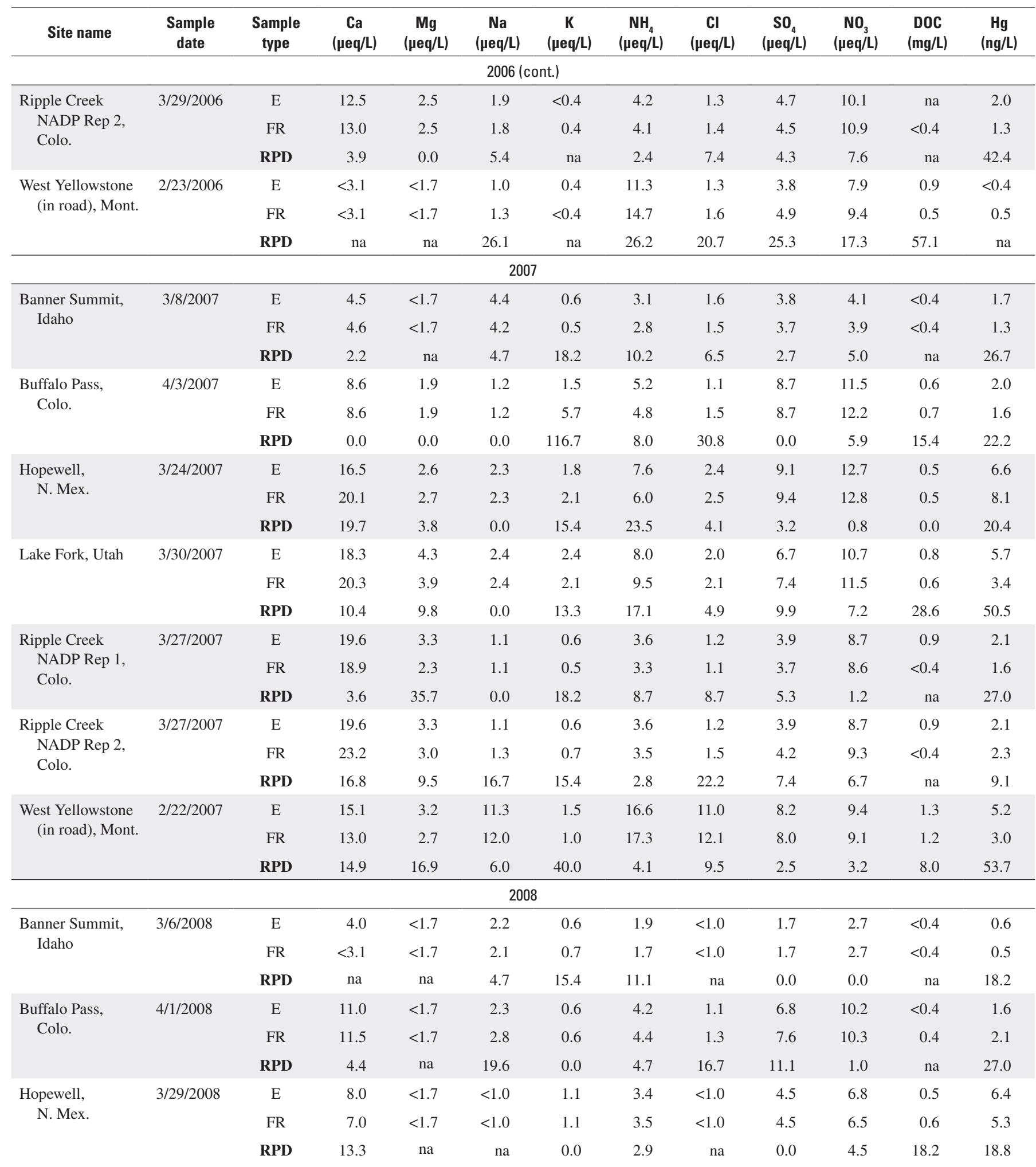


Table 19. Quality-assurance data: relative percent differences in concentrations between environmental and replicate samples for 1993-2008.-Continued

[E, depth-integrated environmental; FR, field replicate; RPD, relative percent difference; $\mu \mathrm{eq} / \mathrm{L}$, microequivalents per liter; $\mathrm{Ca}$, calcium; Mg, magnesium; Na, sodium; K, potassium; $\mathrm{NH}_{4}$, ammonium; $\mathrm{Cl}$, chloride; $\mathrm{SO}_{4}$, sulfate; $\mathrm{NO}_{3}$, nitrate; DOC, dissolved organic carbon; mg/L, milligrams per liter; NADP, National Atmospheric Deposition Program; Hg, mercury; ng/L, nanograms per liter; na, not analyzed; <, below reporting limits; NP, National Park]

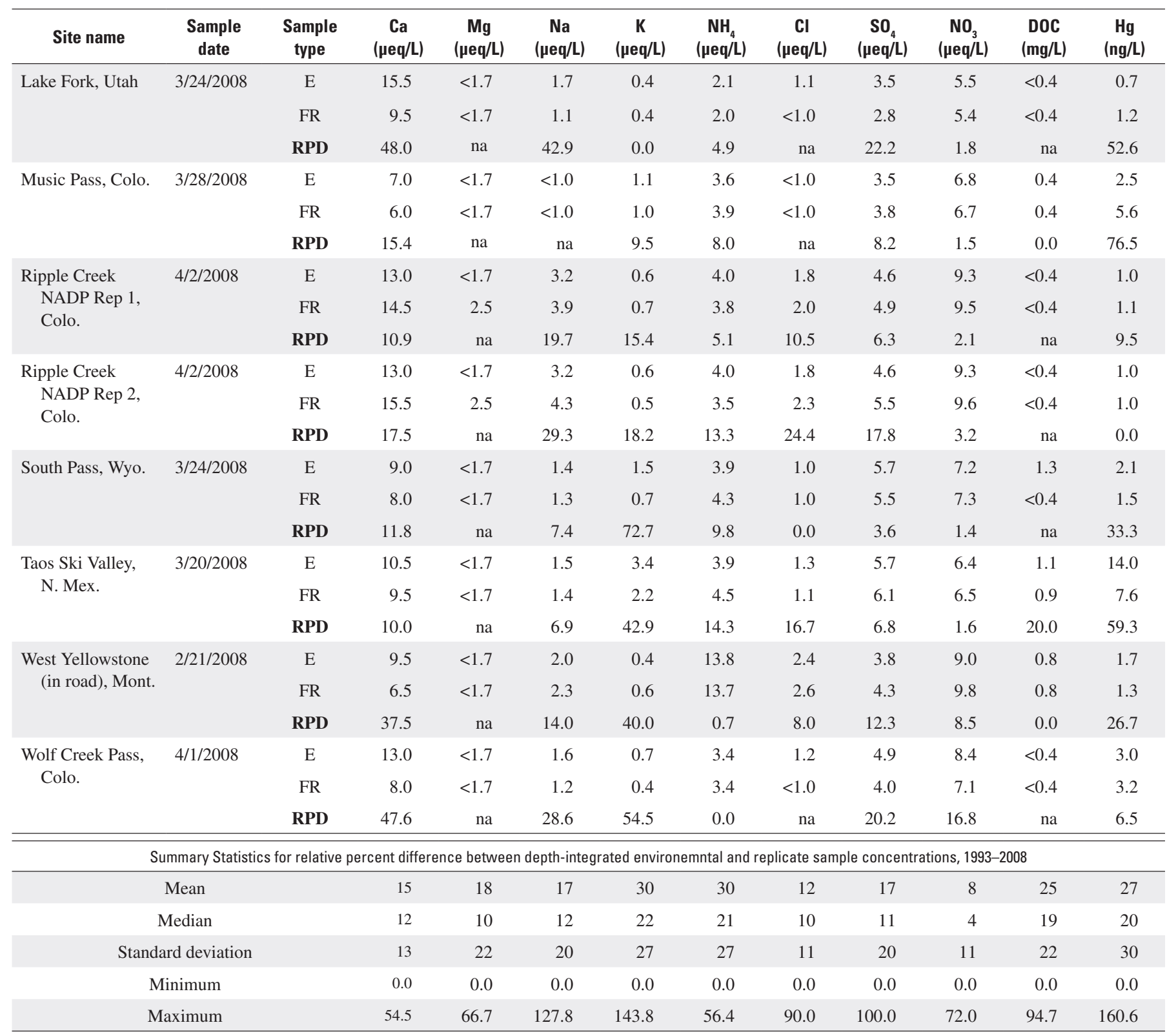




\section{Acknowledgments}

The authors are grateful for the cooperation from numerous individuals from many organizations including the National Park Service and the U.S. Department of Agriculture Forest Service. The authors thank many individuals for their assistance with logistical support, field work, and analytical processing: Stan Bones, Cindy Bosco, Jay Dorr, Dan Fagre, Ben Glass, Bob Hammer, Mary Hektner, Karen Holzer, Nan Ingersoll, Cyndi Kester, Craig McClure, Lisa McKeon, Rick Neam, Gary Nelson, Ted Porwoll, Blaze Reardon, Don Rosenberry, Orville Rosenberry, John Sacklin, Mark Story, and Terry Svalberg. Special thanks also to the following ski resorts who provided generous logistical support: The Big Mountain, Big Sky, Loveland, Showdown, Snow Bowl, Taos Ski Valley, and Teton Village.

\section{References Cited}

Childress, C.J.Q., Foreman, W.T., Connor, B.F., Mahoney, T.J., 1999, U.S. Geological Survey Open-File Report 99-193, 19 p. http://water.usgs.gov/owq/OFR_99-193/procedure. html\#procedure

Ingersoll, G.P., Turk, J.T., Mast, M.A., Clow, D.W., Campbell, D.H., and Bailey, Z.C., 2002, Rocky Mountain snowpack chemistry network: History, methods, and the importance of monitoring mountain ecosystems: U.S. Geological Survey Open-File Report 2001-466, 14 p. http://pubs.usgs.gov/ of/2001/ofro1-466/

Ingersoll, G.P., Mast, M.A., Nanus, Leora, Manthorne, D.J., Hultstrand, D.M., and Winterringer, J., 2005, Rocky Mountain snowpack chemistry at selected sites, 2003: U.S. Geological Survey Open-File Report 2005-1332, 17 p. http:// pubs.usgs.gov/of/2005/1332/.
Mast, M.A., Turk, J.T., Ingersoll, G.P., Clow, D.W., and Kester, Cindy, 2001, Use of stable sulfur isotopes to identify sources of sulfate in Rocky Mountain snowpacks: Atmospheric Environment, v. 35, p. 3303-3313.

Mast, M.A., Campbell, D.H., and Ingersoll, G.P., 2005, Effects of emission reductions at the Hayden powerplant on precipitation, snowpack, and surface-water chemistry in the Mount Zirkel Wilderness Area, Colorado, 1995-2003: U.S. Geological Survey Scientific Investigations Report 2005-5167, $37 \mathrm{p}$.

National Atmospheric Deposition Program (NRSP-3), 2001, Quality assurance report, National Atmospheric Deposition Program, 1999: NADP QA Report 2001-01, Illinois State Water Survey, 2204 Griffith Drive, Champaign, IL 61820.

National Atmospheric Deposition Program (NRSP-3), 2005, 2003 Annual and seasonal data summary for site CO97, part 2-Statistical summary of precipitation chemistry for valid samples: NADP Program Office, Illinois State Water Survey, 2204 Griffith Dr., Champaign, IL 61820 accessed November 2008 at http://nadp.sws.uiuc.edu/ads/2003/ CO97.pdf

National Atmospheric Deposition Program (NRSP-3), 2006, quality assurance plan, version 2.0, June 1, 2006: Central Analytical Laboratory, National Atmospheric Deposition Program. Illinois State Water Survey, 2204 Griffith Dr., Champaign, IL 61820. http://nadp.sws.uiuc.edu/lib/

Turk, J.T., Taylor, H.E., Ingersoll, G.P., Tonnessen, K.A., Clow, D.W., Mast, M.A., Campbell, D.H., and Melack, J.M., 2001, Major-ion chemistry of the Rocky Mountain snowpack, USA: Atmospheric Environment, v. 35, p. 3957-3966.

Publishing support provided by:

Denver Publishing Service Center

For more information concerning this publication, contact:

Director, USGS Colorado Water Science Center

Box 25046, Mail Stop 415

Denver, C0 80225

(303)236-4882

Or visit the Colorado Water Science Center Web site at: http://co.water.usgs.gov/ 
